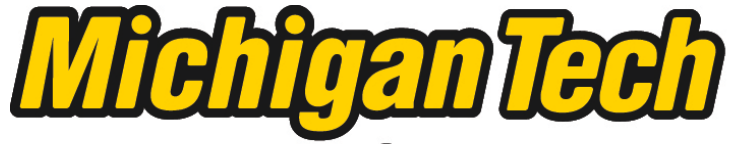 \\ Michigan Technological University Create the Future Digital Commons @ Michigan Tech
}

Dissertations, Master's Theses and Master's Reports - Open

Dissertations, Master's Theses and Master's

Reports

2012

\section{Heat transfer in microwave heating}

Zhiwei Peng

Michigan Technological University

Follow this and additional works at: https://digitalcommons.mtu.edu/etds

Part of the Engineering Science and Materials Commons

Copyright 2012 Zhiwei Peng

\section{Recommended Citation}

Peng, Zhiwei, "Heat transfer in microwave heating", Dissertation, Michigan Technological University, 2012. https://doi.org/10.37099/mtu.dc.etds/17

Follow this and additional works at: https://digitalcommons.mtu.edu/etds

Part of the Engineering Science and Materials Commons 


\title{
HEAT TRANSFER IN MICROWAVE HEATING
}

By

Zhiwei Peng

\begin{abstract}
A DISSERTATION
Submitted in partial fulfillment of the requirements for the degree of DOCTOR OF PHILOSOPHY

(Materials Science and Engineering)
\end{abstract}

MICHIGAN TECHNOLOGICAL UNIVERSITY

2012

(C) 2012 Zhiwei Peng 
This dissertation, "Heat Transfer in Microwave Heating”, is hereby approved in partial fulfillment of the requirements for the Degree of DOCTOR OF PHILOSOPHY IN MATERIALS SCIENCE AND ENGINEERING.

Department of Materials Science and Engineering

Signatures:

Dissertation Advisor

Jiann-Yang Hwang

Department Chair

Stephen L. Kampe

Date 
To My Parents 


\section{Table of Contents}

List of Figures ........................................................................................................................ ix

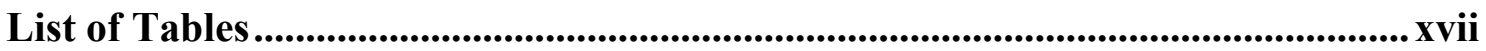

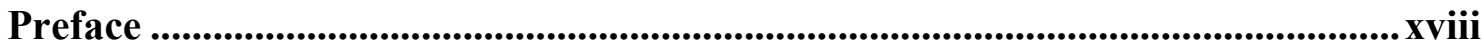

Acknowledgements ............................................................................................................... $\mathrm{xx}$

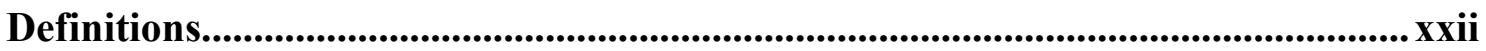

List of Abbreviations ................................................................................................ xxvii

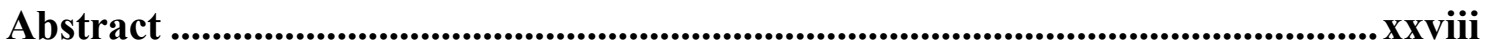

Chapter 1 Introduction ............................................................................................... 1

1.1 Microwave Processing of Materials............................................................... 1

1.2 Microwave Assisted Ironmaking and Steelmaking............................................ 2

1.3 Microwave Heating Fundamentals....................................................................... 4

1.3.1 Permittivity and Permeability............................................................. 4

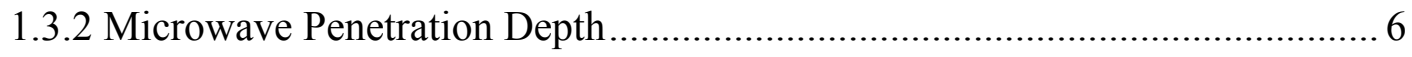

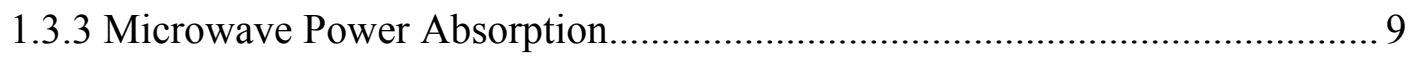

1.3.4 Microwave Propagation in Materials .................................................. 10

1.3.5 Characterization of Microwave Absorption Properties of Materials ............. 12

1.4 Heat Transfer Analysis of Microwave Heating ................................................. 18

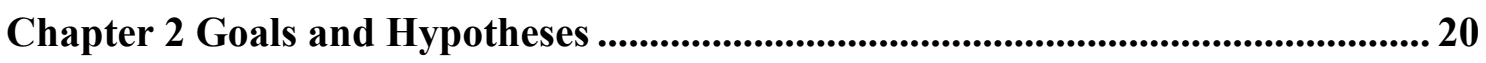

2.1 Derive New Equations for Characterizing Microwave Decay ....................... 20

2.2 Identify the Contribution of Magnetic Loss to Microwave Heating ............... 21 
2.3 Investigate Microwave Absorption Properties of Materials for Ironmaking 21

2.4 Model Microwave Propagation in Dielectric Media......................................... 22

2.5 Simulate Heat Transfer in Microwave Heating .............................................. 23

2.6 Improve Microwave Absorption and Heating Uniformity .............................23

Chapter 3 New Equations for Characterizing Microwave Decay.............................. 25

3.1 Derivation of Microwave Power Penetration Depth Equation ........................ 25

3.2 Derivation of Field Attenuation Length Equation .......................................... 28

3.3 Derivation of Half-power Depth Equation ...................................................29

Chapter 4 Magnetic Loss in Microwave Heating ...................................................... 30

4.1 Magnetic Loss and Heat Generation ............................................................ 30

4.2 Derivation of Microwave Power Dissipation Equation ...................................... 32

4.3 Derivation of Magnetic Loss Equation................................................................ 34

Chapter 5 Microwave Absorption Properties of Materials for Ironmaking .......... 44

5.1 Hematite ............................................................................................................................. 45

5.1.1 Permittivity and Permeability of Hematite............................................. 45

5.1.2 Microwave Absorption Capability of Hematite ........................................ 52

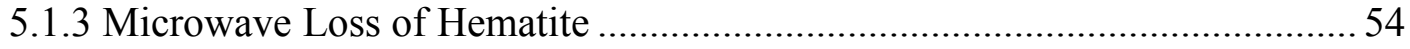

5.2 Magnetite Concentrate ............................................................................................56

5.2.1 Permittivity and Permeability of Magnetite Concentrate .............................. 56

5.2.2 Microwave Absorption Capability of Magnetite Concentrate ..................... 61

5.2.3 Microwave Loss of Magnetite Concentrate ............................................ 62

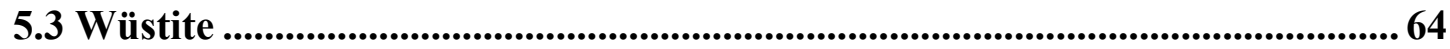




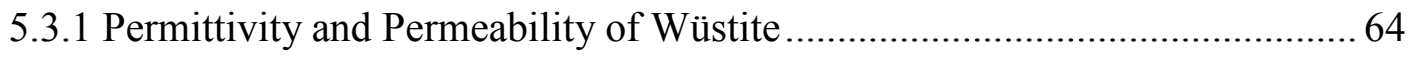

5.3.2 Microwave Absorption Capability of Wüstite .............................................. 72

5.3.3 Microwave Loss of Wüstite ....................................................................... 74

5.3.4 Kinetics of Wüstite Decomposition ............................................................ 75

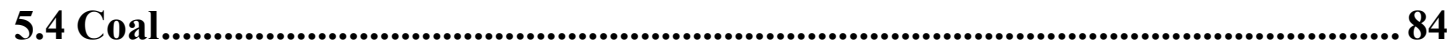

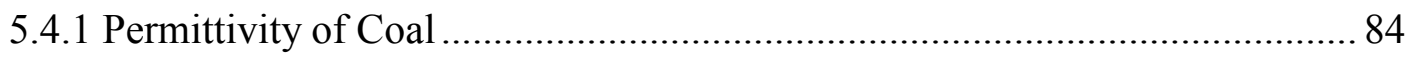

5.4.2 Microwave Absorption Capability of Coal …………………….................. 92

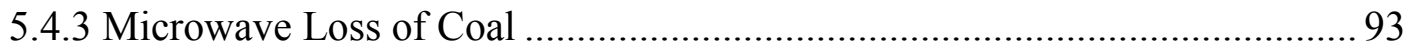

Chapter 6 Microwave Propagation Behaviors in Dielectric Media ............................ 94

6.1 FDTD Method ..................................................................................................................94

6.2 Formulations of the FDTD Algorithm .................................................................96

6.3 Modeling of Microwave Propagation in Various Media .......................................99

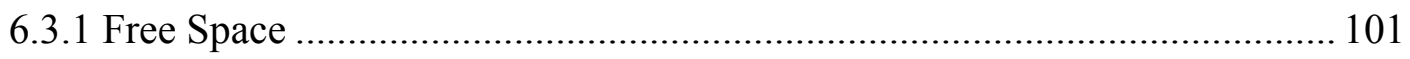

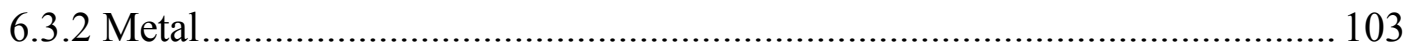

6.3.3 Non-lossy Dielectric Medium ................................................................... 104

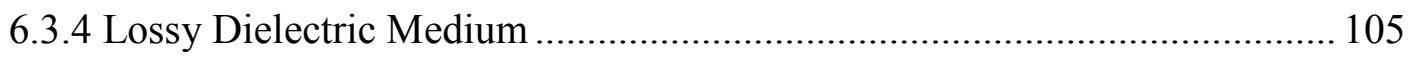

6.3.5 Lossy Magnetic Dielectric without Magnetic loss ......................................... 106

6.3.6 Lossy Magnetic Dielectric with Magnetic loss .............................................. 107

Chapter 7 Simulation of Heat Transfer in Microwave Heating................................. 110

7.1 One-dimensional Simulation ...................................................................................... 110

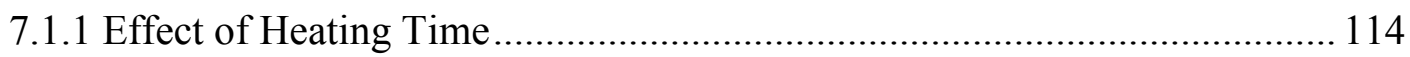

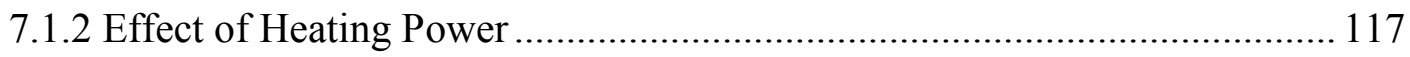


7.1.3 Effect of Microwave Frequency......

7.1.4 Effect of Object Dimension

7.2 Two-dimensional Simulation .................................................................................... 122

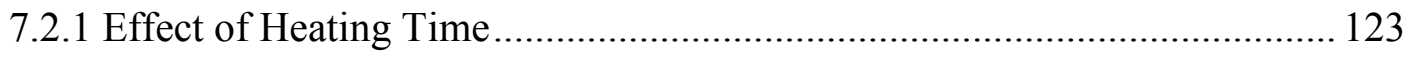

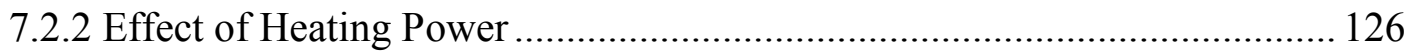

7.2.3 Effect of Microwave Frequency ................................................................. 127

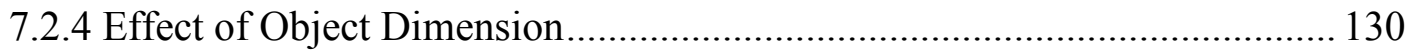

Chapter 8 Dimension Optimization for Absorbers in Microwave Heating ........... 133

8.1 Effect of Absorber Dimension on Microwave Heating...................................... 133

8.2 Microwave Heat Generation and Heat Transfer ............................................... 134

8.3 Dimension Optimization using Reflection Loss .................................................. 136

Chapter 9 Absorber Impedance Matching in Microwave Heating ........................ 143

9.1 Perfect Impedance Matching ...................................................................................... 143

9.2 Derivation of the Function for Evaluating Impedance Matching .................. 145

9.3 Perfect Impedance Matching Map .......................................................................... 149

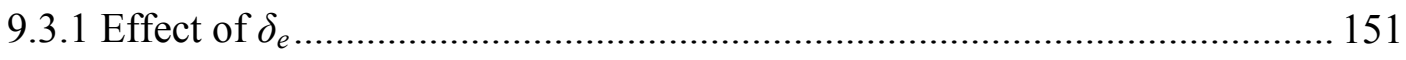

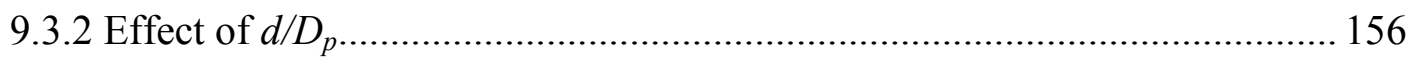

9.3.3 Thickness for Perfect Impedance Matching of Hematite .............................. 162

Chapter 10 Conclusions ........................................................................................................ 171

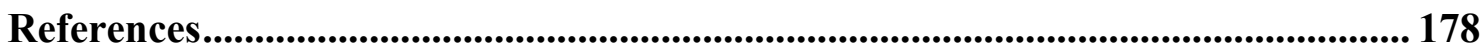

Appendix A-1 Copyright Permission for Chapter 3..................................................... 199

Appendix A-2 Copyright Permission for Chapter 3...................................................... 201 
Appendix B-1 Copyright Permission for Chapter 4 ............................................ 203

Appendix C-1 Copyright Permission for Chapter 5.................................................. 206

Appendix C-2 Copyright Permission for Chapter 5................................................ 208

Appendix C-3 Copyright Permission for Chapter 5.......................................... 210

Appendix C-4 Copyright Permission for Chapter 5............................................... 212

Appendix C-5 Copyright Permission for Chapter 5............................................ 214

Appendix D-1 Copyright Permission for Chapter 6........................................... 224

Appendix E-1 Copyright Permission for Chapter 7 ................................................. 226

Appendix E-2 Copyright Permission for Chapter 7 ................................................ 228

Appendix F-1 Copyright Permission for Chapter 8 ............................................ 230

Appendix G-1 Copyright Permission for Chapter 9 ................................................. 232 


\section{List of Figures}

Fig. 1.1. MTU microwave steelmaking rotary hearth system.

Fig. 1.2. Schematic of the $\mathrm{TM}_{0 \mathrm{n} 0}$ cavity system (in cross-section) showing the linear actuator with the quartz sample holder and a sample located on axis in the center of

the cavity. 17

Fig. 4.1. A magnetic dielectric layer subjected to microwaves from the left side. 34

Fig. 4.2. (a) Electric field and (b) magnetic field distributions for microwave heating of the $0.05-\mathrm{m}$-thick ferrite slabs.

Fig. 4.3. (a) Dielectric loss and (b) magnetic loss distributions for microwave heating of the 0.05 -m-thick ferrite slabs. 42

Fig. 5.1. XRD pattern of $\mathrm{Fe}_{2} \mathrm{O}_{3}$

Fig. 5.2. Field emission-scanning electron microscope (FE-SEM) image of $\mathrm{Fe}_{2} \mathrm{O}_{3}$ particles.

Fig. 5.3. Temperature dependence of complex relative permeability $\left(\varepsilon_{r}{ }^{\prime}\right.$ and $\left.\varepsilon_{r}{ }^{\prime \prime}\right)$ of

$\mathrm{Fe}_{2} \mathrm{O}_{3}$. 48

Fig. 5.4. Variation of bulk density of the sample pellet with temperature during the measurement.

Fig. 5.5. FE-SEM image of the sintered sample pellet. 50

Fig. 5.6.Temperature dependence of complex relative permeability $\left(\mu_{r}{ }^{\prime}\right.$ and $\left.\mu_{r}{ }^{\prime \prime}\right)$ of $\mathrm{Fe}_{2} \mathrm{O}_{3}$. 
Fig. 5.7. Calculated microwave penetration depth of $\mathrm{Fe}_{2} \mathrm{O}_{3}$ as a function of temperature.

Fig. 5.8. Dielectric loss distributions for microwave heating of the $0.05-\mathrm{m}$-thick hematite slabs. 55

Fig. 5.9. Magnetic loss distributions for microwave heating of the $0.05-\mathrm{m}$-thick hematite slabs.

Fig. 5.10. X-ray diffraction pattern of magnetite concentrate. 57

Fig. 5.11. Temperature dependence of complex relative permittivity of magnetite concentrate.

Fig. 5.12. Temperature dependence of complex relative permeability of magnetite concentrate.

Fig. 5.13. Temperature dependence of microwave penetration depth of magnetite concentrate.

Fig. 5.14. Dielectric loss distributions for microwave heating of the 0.05-m-thick magnetite concentrate slabs.

Fig. 5.15. Magnetic loss distributions for microwave heating of the $0.05-\mathrm{m}$-thick magnetite concentrate slabs 63

Fig. 5.16. RT-XRD pattern of the sample: $\mathrm{w}-\mathrm{Fe}_{0.925} \mathrm{O}$. 66

Fig. 5.17. Variation of complex relative permittivity of the sample as a function of temperature. 67

Fig. 5.18. FE-SEM image of $\mathrm{Fe}_{0.925} \mathrm{O}$ particles in $0.5-1.0 \mu \mathrm{m}$ size accumulating together and forming interstices between them. 67 
Fig. 5.19. HT-XRD patterns of the sample at various temperatures: $\mathrm{w}-\mathrm{Fe}_{0.925} \mathrm{O}$,

$$
\mathrm{m}-\mathrm{Fe}_{3} \mathrm{O}_{4} \text {, and } \mathrm{I}-\mathrm{Fe} .
$$

Fig. 5.20. FE-SEM image of $\mathrm{Fe}_{0.925} \mathrm{O}$ after the dielectric measurement.

Fig. 5.21. Variation of complex relative permeability of the sample as a function of temperature.

Fig. 5.22. Microwave penetration depth of $\mathrm{Fe}_{0.925} \mathrm{O}$ in the temperature range of 20 to $200{ }^{\circ} \mathrm{C}$

Fig. 5.23. Microwave penetration depth of $\mathrm{Fe}_{0.925} \mathrm{O}$ at temperatures between 550 and $1100{ }^{\circ} \mathrm{C}$ 74

Fig. 5.24. Dielectric loss distributions for microwave heating of the 0.05 -m-thick wüstite slabs. 75

Fig. 5.25. Variation of decomposition degree of $\mathrm{Fe}_{0.925} \mathrm{O}$ with temperature. 82

Fig. 5.26. Coats-Redfern plot for the decomposition of $\mathrm{Fe}_{0.925} \mathrm{O}$ using the KomatsuUemura equation $\left(D_{6}\right)$.

Fig. 5.27. Temperature dependences of dielectric properties of the pressed coal pellets under UHP argon, at 915 and $2450 \mathrm{MHz}$. Insets: magnification patterns as temperature varies from 24 to $600{ }^{\circ} \mathrm{C}$. The initial pellet density is $1.11 \mathrm{~g} \mathrm{~cm}^{-3}$ and the final density is $1.02 \mathrm{~g} \mathrm{~cm}^{-3}$. The mass loss is $43 \%$ of the initial mass (TGA)... 87

Fig. 5.28. FTIR spectra of the coal at $24^{\circ} \mathrm{C}, 250^{\circ} \mathrm{C}, 550{ }^{\circ} \mathrm{C}, 650^{\circ} \mathrm{C}, 750^{\circ} \mathrm{C}$, and 850 ${ }^{\circ} \mathrm{C}$. 88

Fig. 5.29. FTIR spectra of the coal at $250^{\circ} \mathrm{C}, 650{ }^{\circ} \mathrm{C}$, and $750{ }^{\circ} \mathrm{C}$ 90 
Fig. 5.30. XRD patterns of the coal showing the sharp peaks of quartz $\left(\mathrm{SiO}_{2}\right.$, denoted by “Q”).

Fig. 5.31. Variations of microwave penetration depth of the coal with temperature at 915 and $2450 \mathrm{MHz}$ 92

Fig. 5.32. Dielectric loss distributions for microwave heating of the $0.05-\mathrm{m}$-thick coal slabs. 93

Fig. 6.1. Yee cell in FDTD method. 96

Fig. 6.2. Geometry of space domain ( 800 cells) in the simulation. 100

Fig. 6.3. Electric field distribution in free space $(\mathrm{N}=400)$. 101

Fig. 6.4. Magnetic field distribution in free space $(\mathrm{N}=400)$. 102

Fig. 6.5. Electric field distribution in free space $(\mathrm{N}=5400)$ 102

Fig. 6.6. Magnetic field distribution in free space $(\mathrm{N}=5400)$. 103

Fig. 6.7. Electric field distribution in metal $(\mathrm{N}=5400)$. 103

Fig. 6.8. Magnetic field distribution in metal $(\mathrm{N}=5400)$. 104

Fig. 6.9. Electric field distribution in the non-lossy dielectric medium $(\mathrm{N}=5400) \ldots 104$

Fig. 6.10. Magnetic field distribution in the non-lossy dielectric medium $(\mathrm{N}=5400) .105$

Fig. 6.11. Electric field distribution in the lossy dielectric medium $(\mathrm{N}=5400) \ldots \ldots \ldots .105$

Fig. 6.12. Magnetic field distribution in the lossy dielectric medium $(\mathrm{N}=5400) \ldots \ldots 106$

Fig. 6.13. Electric field distribution in the lossy magnetic dielectric without magnetic $\operatorname{loss}(\mathrm{N}=5400)$. 106

Fig. 6.14. Magnetic field distribution in the lossy magnetic dielectric without magnetic $\operatorname{loss}(\mathrm{N}=5400)$. 107 
Fig. 6.15. Electric field distribution in the lossy magnetic dielectric with magnetic loss

$$
(\mathrm{N}=5400)
$$

Fig. 6.16. Magnetic field distribution in the lossy magnetic dielectric with magnetic loss

$$
(\mathrm{N}=5400)
$$

Fig. 7.1. Depiction of the slab geometry.

Fig. 7.2. Temperature distributions in the magnetite slab for different microwave heating periods at $915 \mathrm{MHz}: \mathrm{a}-1 \mathrm{~s}, \mathrm{~b}-10 \mathrm{~s}, \mathrm{c}-30 \mathrm{~s}$, and d-60 s. Power: $1 \mathrm{MW}$ $\mathrm{m}^{-2}$; Dimension $(L): 0.2 \mathrm{~m}$

Fig. 7.3. Temperature distributions in the magnetite slab for different microwave heating periods at $915 \mathrm{MHz}: \mathrm{a}-60 \mathrm{~s}, \mathrm{~b}-300 \mathrm{~s}, \mathrm{c}-600 \mathrm{~s}$, and d-1200 s. Power: $1 \mathrm{MW} \mathrm{m}{ }^{-2}$; Frequency: $915 \mathrm{MHz}$; Dimension $(L): 0.2 \mathrm{~m}$. 116

Fig. 7.4. Temperature dependences of magnetite thermal diffusivity $(\alpha)$ and microwave penetration depth $\left(D_{p}\right)$. 116

Fig. 7.5. Temperature distributions in the magnetite slab under different microwave heating powers at $915 \mathrm{MHz}: \mathrm{a}-0.5 \mathrm{MW} \mathrm{m}^{-2}, \mathrm{~b}-1 \mathrm{MW} \mathrm{m} \mathrm{m}^{-2} \mathrm{c}-2 \mathrm{MW} \mathrm{m}^{-2}$, and $\mathrm{d}-4 \mathrm{MW} \mathrm{m} \mathrm{m}^{-2}$. Heating time: $60 \mathrm{~s}$; Dimension $(L): 0.2 \mathrm{~m}$ 118

Fig. 7.6. Temperature distributions in the magnetite slab for different microwave heating periods at $2450 \mathrm{MHz}: \mathrm{a}-1 \mathrm{~s}, \mathrm{~b}-10 \mathrm{~s}, \mathrm{c}-30 \mathrm{~s}$, and d-60 s. Power: $1 \mathrm{MW}$ $\mathrm{m}^{-2}$; Dimension $(L): 0.2 \mathrm{~m}$.

Fig. 7.7. Temperature distributions in the magnetite slab for different microwave heating periods at $2450 \mathrm{MHz}$ : $\mathrm{a}-60 \mathrm{~s}, \mathrm{~b}-300 \mathrm{~s}, \mathrm{c}-600 \mathrm{~s}$, and d-1200 s. Power: $1 \mathrm{MW} \mathrm{m}^{-2}$; Dimension $(L): 0.2 \mathrm{~m}$ 121 
Fig. 7.8. Temperature distributions in the magnetite slab with different dimensions $(L)$ at $2450 \mathrm{MHz}: \mathrm{a}-0.2 \mathrm{~m}, \mathrm{~b}-0.15 \mathrm{~m}, \mathrm{c}-0.1 \mathrm{~m}$, and d-0.05 m. Heating time: $60 \mathrm{~s}$; Power: $1 \mathrm{MW} \mathrm{m}^{-2}$. 122

Fig. 7.9. Depiction of the 2-D object (one-quarter) geometry 123

Fig. 7.10. Temperature $\left({ }^{\circ} \mathrm{C}\right)$ profiles in the 2-D object for different microwave heating periods: (a) $1 \mathrm{~s}$, (b) $60 \mathrm{~s}$, (c) $300 \mathrm{~s}$, and (d) 600 s. Power: $1 \mathrm{MW} \mathrm{m}{ }^{-2}$; Frequency: $915 \mathrm{MHz}$; Dimension (L): $0.2 \mathrm{~m}$. 125

Fig. 7.11. Temperature $\left({ }^{\circ} \mathrm{C}\right)$ profiles in the 2-D object under different microwave heating powers: (a) $0.5 \mathrm{MW} \mathrm{m}^{-2}$, (b) $1 \mathrm{MW} \mathrm{m}^{-2}$, (c) $2 \mathrm{MW} \mathrm{m}^{-2}$, and (d) $4 \mathrm{MW} \mathrm{m}^{-2}$. Heating time: 60 s; Frequency: $915 \mathrm{MHz}$; Dimension $(L): 0.2 \mathrm{~m}$. 127

Fig. 7.12. Temperature $\left({ }^{\circ} \mathrm{C}\right)$ profiles in the 2-D object for different microwave heating periods at $2450 \mathrm{MHz}$ (a) $1 \mathrm{~s}$, (b) $60 \mathrm{~s}$, (c) $300 \mathrm{~s}$, and (d) $600 \mathrm{~s}$. Power: $1 \mathrm{MW} \mathrm{m}^{-2}$; Dimension $(L): 0.2 \mathrm{~m}$. 129

Fig. 7.13. Temperature $\left({ }^{\circ} \mathrm{C}\right)$ profiles in the 2-D object with different dimensions: (a) 0.2 m, (b) $0.15 \mathrm{~m}$, (c) $0.1 \mathrm{~m}$, and (d) $0.05 \mathrm{~m}$. Heating time: $60 \mathrm{~s}$; Power: $1 \mathrm{MW} \mathrm{m}^{-2}$; Frequency: $2450 \mathrm{MHz}$

Fig. 8.1. Schematic of an absorber under microwave irradiation. 138

Fig. 8.2. (a) Complex relative permittivity of hematite vs. temperature at 915 and 2450 MHz. (b) Dielectric loss tangent of hematite vs. temperature at 915 and $2450 \mathrm{MHz}$. 
Fig. 8.3. High-temperature microwave absorption of hematite: (a) Calculated reflection loss vs. temperature at $915 \mathrm{MHz}$. (b) Calculated reflection loss vs. temperature at $2450 \mathrm{MHz}$ 141

Fig. 9.1. Impedance matching map for a dielectric absorber (3-D view). 149

Fig. 9.2. Impedance matching map for a dielectric absorber (top viewpoint). 150

Fig. 9.3. Impedance matching map for a dielectric absorber (right viewpoint). 150

Fig. 9.4. Impedance matching map for a dielectric absorber (front viewpoint). 151

Fig. 9.5. $f(d)$ vs. $d / D_{p}$ ranging from 0 to $3\left(\delta_{e}=\pi / 24\right)$. 152

Fig. 9.6. $f(d)$ vs. $d / D_{p}$ ranging from 0 to $5\left(\delta_{e}=\pi / 24\right)$. 152

Fig. 9.7. $f(d)$ vs. $d / D_{p}$ ranging from 0 to $3\left(\delta_{e}=\pi / 12\right)$. 153

Fig. 9.8. $f(d)$ vs. $d / D_{p}$ ranging from 0 to $5\left(\delta_{e}=\pi / 12\right)$. 153

Fig. 9.9. $f(d)$ vs. $d / D_{p}$ ranging from 0 to $3\left(\delta_{e}=\pi / 6\right)$. 154

Fig. 9.10. $f(d)$ vs. $d / D_{p}$ ranging from 0 to $5\left(\delta_{e}=\pi / 6\right)$. 154

Fig. 9.11. $f(d)$ vs. $d / D_{p}$ ranging from 0 to $3\left(\delta_{e}=\pi / 4\right)$. 155

Fig. 9.12. $f(d)$ vs. $d / D_{p}$ ranging from 0 to $5\left(\delta_{e}=\pi / 4\right)$. 155

Fig. 9.13. $f(d)$ vs. $d / D_{p}$ ranging from 0 to $3\left(\delta_{e}=3 \pi / 8\right)$. 156

Fig. 9.14. $f(d)$ vs. $d / D_{p}$ ranging from 0 to $5\left(\delta_{e}=3 \pi / 8\right)$. 156

Fig. 9.15. $f(d)$ vs. $\delta_{e}$ ranging from 0 to $\pi / 6\left(d / D_{p}=1\right)$. 157

Fig. 9.16. $f(d)$ vs. $\delta_{e}$ ranging from 0 to $\pi / 2\left(d / D_{p}=1\right)$. 157

Fig. 9.17. $f(d)$ vs. $\delta_{e}$ ranging from 0 to $\pi / 6\left(d / D_{p}=2\right)$. 158

Fig. 9.18. $f(d)$ vs. $\delta_{e}$ ranging from 0 to $\pi / 2\left(d / D_{p}=2\right)$. 158

Fig. 9.19. $\mathrm{f}(\mathrm{d})$ vs. $\delta_{e}$ ranging from 0 to $\pi / 6\left(d / D_{p}=3\right)$. 159 
Fig. 9.20. $f(d)$ vs. $\delta_{e}$ ranging from 0 to $\pi / 2\left(d / D_{p}=3\right)$.

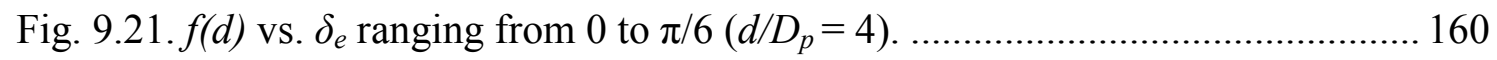

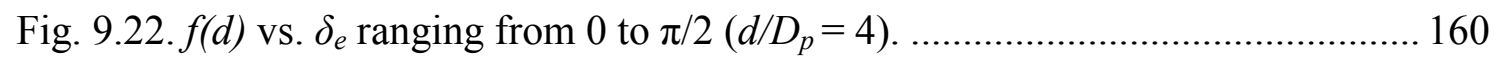

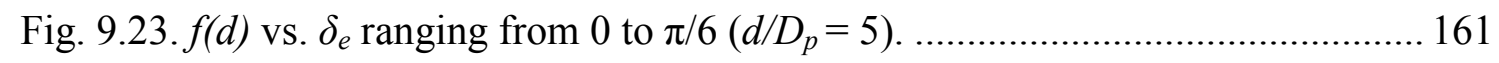

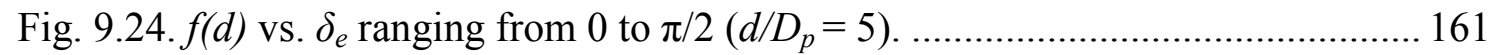

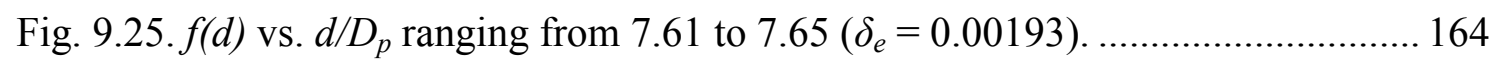

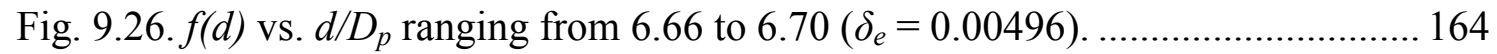

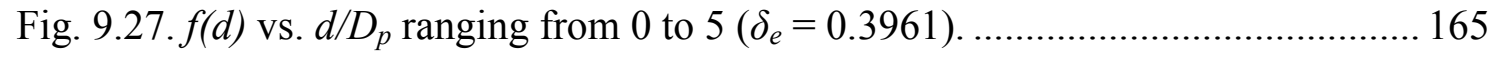

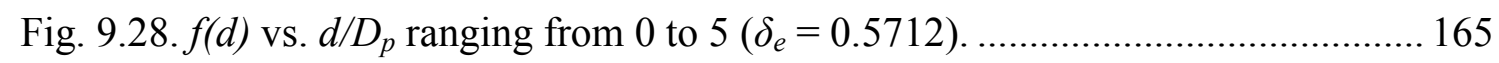

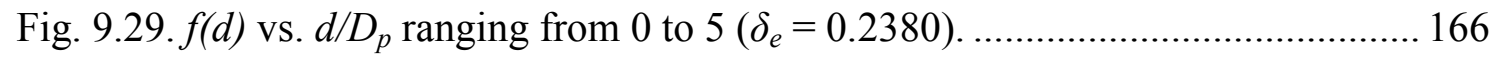

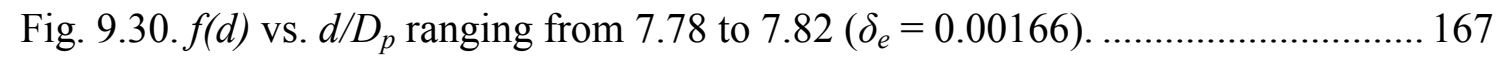

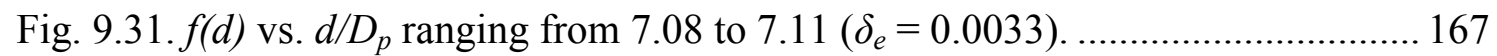

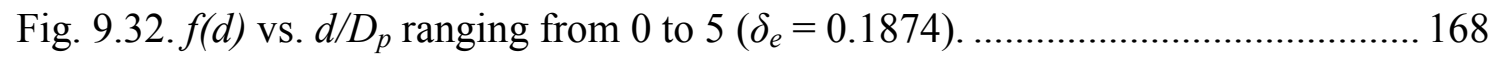

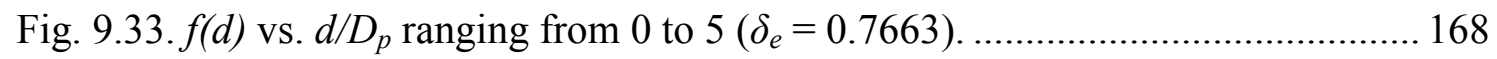

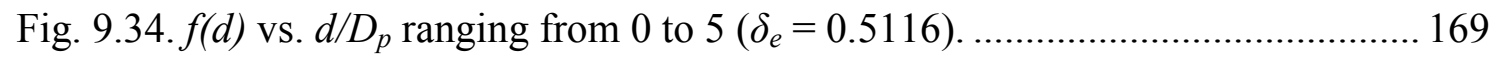




\section{List of Tables}

Table 1.1 Materials classification in microwave heating. ........................................... 10

Table 4.1 Permittivity and permeability of the ferrites at $2-40 \mathrm{GHz}$............................ 39

Table 4.2 Microwave absorption parameters of the ferrites at $2450 \mathrm{MHz}$.................... 40

Table 5.1 Values of constants in the polynomial function (for $\varepsilon_{r}$ and $\mu_{r}$ ) represented by

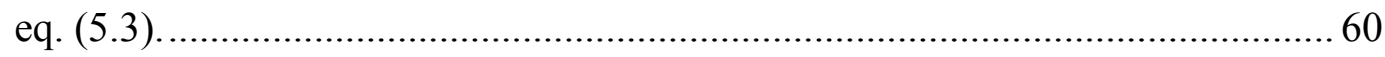

Table 5.2 Values of constants in the polynomial function (for $D_{p}$ ) represented by eq.

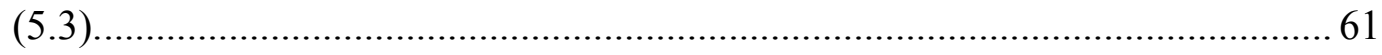

Table 5.3 Algebraic expressions of $f(\boldsymbol{\alpha})$ and $g(\boldsymbol{\alpha})$ for various kinetic models................ 80

Table 5.4 Calculated kinetic parameters of the decomposition of $\mathrm{Fe}_{0.925} \mathrm{O} \ldots \ldots \ldots \ldots \ldots \ldots \ldots \ldots . .83$

Table 7.1 Thermophysical properties and modeling parameters used in the simulation.

Table 9.1 Maximum thickness of hematite for perfect impedance matching............... 162 


\section{Preface}

Microwave energy has been extensively employed in daily life. The various applications of microwave heating have significantly promoted social progress and human development. It has been gaining substantial attention in materials processing over the past three decades. A substantial amount of lab-scale research has identified the characteristic of volumetric heating by microwave energy, which leads to a rapid completion of materials processing. The potential advantages of microwave heating have impelled scientists to continuously study this technique, and to design and implement large heating systems for industrial use.

In the scale-up of microwave heating system, one of the most important issues is precise and active control of heat transfer in the heating process for energy conservation and heating uniformity. It can be addressed by studying the microwave heating mechanism, which has rarely been investigated by most of researchers. This doctoral research was aimed to advance the understanding of heating fundamentals of microwave assisted steelmaking through the investigations on the following aspects: (1) characterization of microwave decay using the derived equations, (2) quantification of magnetic loss, (3) determination of microwave absorption properties of materials, (4) modeling of microwave propagation, (5) simulation of heat transfer, and (6) improvement of microwave absorption and heating uniformity. 
This research led to some publications in refereed journals and conference proceedings, which are primarily included in Chapters 3-9 of the dissertation. The author keeps only his contribution in those chapters. In particular, the author performed all of the calculations and simulations for the work in this dissertation. Readers are referred to the first page of each of those chapters for details.

This research was performed under the supervision of Dr. Jiann-Yang Hwang. Without his encouragement, unfailing guidance, and financial support, it would not have been completed. All his contributions here are highly appreciated. 


\section{Acknowledgements}

First and foremost I would like to express my deepest gratitude to my advisor, Dr. Jiann-Yang Hwang, for his most invaluable guidance, tremendous patience, continuous encouragement, and everlasting support during my Ph.D. pursuit. I gratefully appreciate all his contributions of time, ideas, and funding to make my $\mathrm{Ph} . \mathrm{D}$. experience productive and stimulating. I am so fortunate to have such a great advisor whose enthusiasm for science and kindness to people set the norm for me in my future career.

I would like to sincerely thank my committee members, Dr. Stephen Hackney, Dr. Stephen Kampe, Dr. Elena Semouchkina, and Dr. Song-Lin Yang, for their kindness to review my dissertation. Their valuable advice and constructive criticism broadened my knowledge and helped me substantially in preparing this dissertation.

I have benefited enormously from the generosity and support of many faculty members at Michigan Technological University. In particular, I would like to thank the following professors for sharing their enthusiasm for and valuable comments on my work: Dr. Xiaodi Huang (MSE), Dr. Phillip Merkey (CS), Dr. Abhijit Mukherjee (MEEM), Dr. Xinli Wang (ST), Dr. Yu Wang (MSE), Dr. Yun Hang Hu (MSE), and Dr. Jaroslaw Drelich (MSE). 
I am particularly indebted to my colleagues at Microwave Properties North, Canada, Dr. Ron Hutcheon and Mr. Joe Mouris, whose support made this work possible. Their valuable assistance and helpful discussion throughout the characterization of dielectric properties of materials are greatly appreciated.

I am grateful to my research group members, Wayne Bell, Matthew Andriese, Xiang Sun, Zheng Zhang, Chienyu Wen, Shangzhao Shi, Bowen Li, Allison Hein and other students, for their assistance throughout my research.

I would like to thank Stephen Forsell, Owen Mills, Edward Laitila, and Ruth Kramer for their technical support in materials preparation and characterizations.

I would also like to extend my appreciation to my friends, Zhonghai Wang, Lihui Hu, Xiaoliang Zhong, Mimi Yang, Xuan Li, Sanchai Kuboon, Pubodee Ratana-arsanarom, Parawee Pumwongpitak, Suntara Fueangfung, Yuenyong Nilsiam, Siranee Nuchitprasitchai, Patcharapol Gorgitrattanagul, Andrew Baker, Chong-Lyuck Park, JenYung Chang, Lei Zhang, Junqing Zhang, Tianle Cheng, Yan Yang, Jie Zhou, and Jiesheng Wang, for their help and support during my Ph.D. study at Michigan Tech.

Finally, I must acknowledge with tremendous and deep thanks my dearest parents, my elder brother, sister in law and my lovely niece. Without their unending support and encouragement, this work could not have been finished and succeeded. 


\section{Definitions}

\begin{tabular}{|c|c|c|}
\hline Symbol & Definition & Unit \\
\hline \multicolumn{3}{|c|}{ Greek symbols } \\
\hline$\alpha$ & field attenuation factor & $\mathrm{Np} \mathrm{m^{-1 }}$ \\
\hline$\alpha$ & decomposition degree & None \\
\hline$\alpha^{*}$ & heat diffusivity & $m^{-2} s^{-1}$ \\
\hline$\beta$ & phase constant & $\operatorname{rad~m^{-1}}$ \\
\hline$\beta^{*}$ & heating rate & $\mathrm{K} \min ^{-1}$ \\
\hline$\gamma$ & propagation constant & $\mathrm{m}^{-1}$ \\
\hline$\delta$ & phase angle of reflection coefficient & rad \\
\hline$\varepsilon$ & permittivity & $\mathrm{F} \mathrm{m}^{-1}$ \\
\hline$\varepsilon^{\prime}$ & real part of complex permittivity & $\mathrm{F} \mathrm{m}^{-1}$ \\
\hline$\varepsilon$ & emissivity & None \\
\hline$\varepsilon_{0}$ & permittivity of free space & $\mathrm{F} \mathrm{m}^{-1}$ \\
\hline$\varepsilon_{r}$ & complex relative permittivity & None \\
\hline$\varepsilon_{r}^{\prime}$ & real part of complex relative permittivity & None \\
\hline$\varepsilon_{r, d^{\prime}}$ & displacement current contribution to the real part of & None \\
\hline & complex relative permittivity & \\
\hline$\varepsilon_{r}^{\prime \prime}$ & imaginary part of complex relative permittivity & None \\
\hline$\varepsilon_{r, d^{\prime \prime}}$ & displacement current contribution to the imaginary part of & None \\
\hline
\end{tabular}


complex relative permittivity

\begin{tabular}{|c|c|c|}
\hline$\eta$ & impedance & $\Omega$ \\
\hline$\kappa$ & thermal conductivity & $\mathrm{W} \mathrm{K}^{-1} \mathrm{~m}^{-1}$ \\
\hline$\lambda_{0}$ & microwave wavelength in free space & $\mathrm{m}$ \\
\hline$\mu$ & permeability & $\mathrm{H} \mathrm{m}^{-1}$ \\
\hline$\mu^{\prime}$ & real part of complex permeability & $\mathrm{H} \mathrm{m}^{-1}$ \\
\hline$\mu_{0}$ & permeability of free space & $\mathrm{H} \mathrm{m}^{-1}$ \\
\hline$\mu_{r}$ & complex relative permeability & None \\
\hline$\mu_{r}^{\prime}$ & real part of complex relative permeability & None \\
\hline$\mu_{r}^{\prime \prime}$ & imaginary part of complex relative permeability & None \\
\hline$\rho$ & density & $\mathrm{kg} \mathrm{m}^{-3}$ \\
\hline$\sigma$ & electric conductivity & $\mathrm{S} \mathrm{m}^{-1}$ \\
\hline$\sigma$ & Stefan-Boltzmann constant & $\mathrm{W} \mathrm{m}{ }^{-2} \mathrm{~K}^{-4}$ \\
\hline$\sigma^{*}$ & equivalent magnetic loss & $\Omega \mathrm{m}^{-1}$ \\
\hline$\tau$ & phase angle of transmission coefficient & $\mathrm{rad}$ \\
\hline$\chi_{e}$ & electric susceptibility & None \\
\hline$\chi_{m}$ & magnetic susceptibility & None \\
\hline$\omega$ & microwave angular frequency & $\operatorname{rad~s}^{-1}$ \\
\hline
\end{tabular}

Nomenclature

$\begin{array}{lll}A & \text { real calibration constant } & \text { None } \\ A^{*} & \text { frequency factor } & \mathrm{min}^{-1} \\ c_{p} & \text { specific heat capacity } & \mathrm{J} \mathrm{kg}^{-1} \mathrm{C}^{-1}\end{array}$




\begin{tabular}{|c|c|c|}
\hline$d$ & absorber thickness & $\mathrm{m}$ \\
\hline$D$ & diameter & $\mathrm{m}$ \\
\hline$D_{f}$ & field attenuation length & $\mathrm{m}$ \\
\hline$D_{h}$ & half-power depth & $\mathrm{m}$ \\
\hline$D_{p}$ & penetration depth & $\mathrm{m}$ \\
\hline E & electric field strength & $\mathrm{V} \mathrm{m}^{-1}$ \\
\hline$E^{*}$ & complex conjugate of electric field strength & $\mathrm{V} \mathrm{m}^{-1}$ \\
\hline$E_{a}$ & activation energy & $\mathrm{kJ} \mathrm{mol}^{-1}$ \\
\hline$\tilde{E}$ & normalized electric field strength & None \\
\hline$f$ & frequency & $\mathrm{Hz}$ \\
\hline$f_{e}$ & $\begin{array}{l}\text { specific cavity mode frequency in the permittivity } \\
\text { measurement }\end{array}$ & $\mathrm{Hz}$ \\
\hline$f_{m}$ & $\begin{array}{l}\text { specific cavity mode frequency in the permeability } \\
\text { measurement }\end{array}$ & $\mathrm{Hz}$ \\
\hline$\Delta f$ & frequency shift produced by the sample & $\mathrm{Hz}$ \\
\hline$F_{\text {sh }}$ & real number dependent on the sample shape & None \\
\hline$\Delta G_{m}{ }^{\neq}$ & $\begin{array}{l}\text { Gibbs free energy at temperature of the maximal rate of } \\
\text { decomposition }\end{array}$ & $\mathrm{kJ} \mathrm{mol}^{-1}$ \\
\hline$h$ & heat transfer coefficient & $\mathrm{W} \mathrm{m}{ }^{-2}{ }^{\circ} \mathrm{C}^{-1}$ \\
\hline$h^{*}$ & Planck's constant & $\mathrm{J} \mathrm{s}$ \\
\hline$H$ & magnetic field strength & $\mathrm{A} \mathrm{m}^{-1}$ \\
\hline$H^{*}$ & complex conjugate of magnetic field strength & $\mathrm{A} \mathrm{m}^{-1}$ \\
\hline
\end{tabular}




\begin{tabular}{|c|c|c|}
\hline$\Delta H^{\ddagger}$ & enthalpy of activation & $\mathrm{kJ} \mathrm{mol}^{-1}$ \\
\hline$i$ & index of mesh point along the $x$ direction & None \\
\hline$j$ & imaginary unit & None \\
\hline$J$ & electric current density & $\mathrm{A} \mathrm{m}^{-2}$ \\
\hline$k$ & rate constant & $\min ^{-1}$ \\
\hline$k_{B}$ & Boltzmann constant & $\mathrm{J} \mathrm{K}^{-1}$ \\
\hline$l$ & length & $\mathrm{m}$ \\
\hline$L$ & half object thickness & $\mathrm{m}$ \\
\hline$m$ & number of mesh grid along the $x$ direction & None \\
\hline$M$ & magnetic current density & $\mathrm{A} \mathrm{m}^{-2}$ \\
\hline$n$ & number of time grid & None \\
\hline$N$ & index of time period & None \\
\hline$P$ & instantaneous power absorbed per unit volume & $\mathrm{W} \mathrm{m}{ }^{-3}$ \\
\hline$P_{0}$ & heating generation at sample surface & $\mathrm{MW} \mathrm{m} \mathrm{m}^{-2}$ \\
\hline$P(x)$ & heat generation & $\mathrm{MW} \mathrm{m}^{-3}$ \\
\hline$Q$ & quality factor & None \\
\hline$Q_{E}$ & dielectric loss & $\mathrm{W} \mathrm{m}^{-3}$ \\
\hline$Q_{H}$ & magnetic loss & $\mathrm{W} \mathrm{m}^{-3}$ \\
\hline$Q_{L E}$ & loaded cavity quality factor with the empty holder & None \\
\hline$Q_{L S}$ & loaded cavity quality factor with the holder and sample & None \\
\hline$R$ & resistance & $\Omega$ \\
\hline$R^{*}$ & gas constant & $\mathrm{J} \mathrm{mol}^{-1} \mathrm{~K}$ \\
\hline
\end{tabular}




\begin{tabular}{|c|c|c|}
\hline$R_{r}$ & reflection coefficient & None \\
\hline$s$ & surface area & $\mathrm{m}^{2}$ \\
\hline$S$ & Poynting vector & $\mathrm{W} \mathrm{m} \mathrm{m}^{-2}$ \\
\hline$\Delta S^{\neq}$ & entropy of activation & $\mathrm{J} \mathrm{mol}^{-1} \mathrm{~K}^{-1}$ \\
\hline$t$ & time & $\mathrm{s}$ \\
\hline $\tan \delta$ & loss tangent & None \\
\hline $\tan \delta_{\varepsilon}$ & dielectric loss tangent & None \\
\hline $\tan \delta_{\mu}$ & magnetic loss tangent & None \\
\hline$\Delta t$ & time step & $\mathrm{s}$ \\
\hline$T$ & temperature & ${ }^{\circ} \mathrm{C}$ or $\mathrm{K}$ \\
\hline$T_{0}$ & initial temperature & ${ }^{\circ} \mathrm{C}$ or $\mathrm{K}$ \\
\hline$T_{m}$ & temperature at which the maximum reaction rate occurs & ${ }^{\circ} \mathrm{C}$ or $\mathrm{K}$ \\
\hline$T_{\infty}$ & environmental temperature & ${ }^{\circ} \mathrm{C}$ or $\mathrm{K}$ \\
\hline$T_{t}$ & transmission coefficient & None \\
\hline$V_{c}$ & cavity volume & $\mathrm{m}^{3}$ \\
\hline$V_{s}$ & sample volume & $\mathrm{m}^{3}$ \\
\hline$\Delta x$ & space step along the $x$ direction & $\mathrm{m}$ \\
\hline$\Delta y$ & space step along the $y$ direction & $\mathrm{m}$ \\
\hline$\Delta z$ & space step along the $z$ direction & $\mathrm{m}$ \\
\hline
\end{tabular}




\section{List of Abbreviations}

\begin{tabular}{ll}
\hline Abbreviation & Full name \\
\hline CFDTD & conformal finite-difference time-domain \\
CPMT & cavity perturbation measurement technique \\
EPD & explicit finite-difference \\
FDTD & finite-difference time-domain \\
FE-SEM & field-emission scanning electron microscope \\
FTIR & Fourier transform infrared spectroscopy \\
HT-XRD & high temperature X-ray diffraction \\
RCM & resonant cavity method \\
RL & reflection loss \\
RT-XRD & room-temperature X-ray diffraction \\
TLM & transverse magnetic \\
TGA & thermal gravimetric analysis \\
TEM & \\
TMCPM & \\
&
\end{tabular}




\begin{abstract}
Heat transfer is considered as one of the most critical issues for design and implement of large-scale microwave heating systems, in which improvement of the microwave absorption of materials and suppression of uneven temperature distribution are the two main objectives. The present work focuses on the analysis of heat transfer in microwave heating for achieving highly efficient microwave assisted steelmaking through the investigations on the following aspects: (1) characterization of microwave dissipation using the derived equations, (2) quantification of magnetic loss, (3) determination of microwave absorption properties of materials, (4) modeling of microwave propagation, (5) simulation of heat transfer, and (6) improvement of microwave absorption and heating uniformity.
\end{abstract}

Microwave heating is attributed to the heat generation in materials, which depends on the microwave dissipation. To theoretically characterize microwave heating, simplified equations for determining the transverse electromagnetic mode (TEM) power penetration depth, microwave field attenuation length, and half-power depth of microwaves in materials having both magnetic and dielectric responses were derived. It was followed by developing a simplified equation for quantifying magnetic loss in materials under microwave irradiation to demonstrate the importance of magnetic loss in microwave heating. The permittivity and permeability measurements of various materials, namely, hematite, magnetite concentrate, wüstite, and coal were performed. xxviii 
Microwave loss calculations for these materials were carried out. It is suggested that magnetic loss can play a major role in the heating of magnetic dielectrics.

Microwave propagation in various media was predicted using the finite-difference timedomain method. For lossy magnetic dielectrics, the dissipation of microwaves in the medium is ascribed to the decay of both electric and magnetic fields. The heat transfer process in microwave heating of magnetite, which is a typical magnetic dielectric, was simulated by using an explicit finite-difference approach. It is demonstrated that the heat generation due to microwave irradiation dominates the initial temperature rise in the heating and the heat radiation heavily affects the temperature distribution, giving rise to a hot spot in the predicted temperature profile. Microwave heating at $915 \mathrm{MHz}$ exhibits better heating homogeneity than that at $2450 \mathrm{MHz}$ due to larger microwave penetration depth. To minimize/avoid temperature nonuniformity during microwave heating the optimization of object dimension should be considered.

The calculated reflection loss over the temperature range of heating is found to be useful for obtaining a rapid optimization of absorber dimension, which increases microwave absorption and achieves relatively uniform heating. To further improve the heating effectiveness, a function for evaluating absorber impedance matching in microwave heating was proposed. It is found that the maximum absorption is associated with perfect impedance matching, which can be achieved by either selecting a reasonable sample dimension or modifying the microwave parameters of the sample. 


\section{Chapter 1 Introduction}

\subsection{Microwave Processing of Materials}

Microwaves are electromagnetic waves with wavelengths from $1 \mathrm{~mm}$ to $1 \mathrm{~m}$ and corresponding frequencies between $300 \mathrm{MHz}$ and $300 \mathrm{GHz}{ }^{1}$ Two frequencies, 915 and $2450 \mathrm{MHz}$, are widely used for microwave heating which has gained popularity in the processing of various materials including ceramics, metals and composites. Compared with conventional heating methods, the advantages of microwave heating include time and energy saving, rapid heating rates $\left(>400^{\circ} \mathrm{C} \min ^{-1}\right)$, selective heating, considerably reduced processing time and temperature, unique microstructure and properties, improved product yield, environmental friendliness, and so on. ${ }^{2-5}$

The distinguishing characteristics of microwave heating are attributed to the special heating behavior of microwaves. It delivers heat instantly throughout the materials with volumetric heat generation, resulting in a faster heating rate than conventional heating. ${ }^{6}$ Energy saving and less processing time are thus easy to achieve.

The microwave heating applications involve drying, ${ }^{7,8}$ organic material burnout, 5,9 sintering of ceramics and ceramic composites, ${ }^{10-22}$ polymer processing, ${ }^{23-26}$ joining, ${ }^{27-32}$ melting, ${ }^{33-35}$ synthesis of nanomaterials, ${ }^{36-41}$ etc. It has been demonstrated that microwave heating can significantly reduce the heating time and the energy cost. 
In contrary to the widely experimental applications, the detailed theoretical analysis of the microwave heating process is still highly required. ${ }^{42}$ Because the heating relies on the dielectric and/or magnetic properties of materials, which are in turn affected by the microwave frequencies and temperature, it is more difficult to investigate the heat transfer in microwave heating compared with that in conventional one. To achieve a full understanding of the heating mechanism, the microwave propagation and dissipation behaviors in various media, the dielectric and/or magnetic characterizations of different materials, and the modeling and simulation of heat transfer in microwave heating should be performed.

\subsection{Microwave Assisted Ironmaking and Steelmaking}

Microwave assisted ironmaking has been studied since the early 1990 s. $^{43-46}$ The relation between microwave energy and carbothermic reduction of iron ores was explored by several scientists. It has shown that the main metallurgical reactions in the ironmaking process including the carbothermic reduction of magnetite concentrates and hematite

fines were satisfactorily and rapidly carried out with microwave energy. ${ }^{45,46} \mathrm{~A}$ complete reduction could be achieved after about $15 \mathrm{~min}$ in a microwave oven operating at 2450 $\mathrm{MHz}$ and $1400 \mathrm{~W} \cdot{ }^{46}$ The microwave reduction process at up to $1000{ }^{\circ} \mathrm{C}$ was probably superior to conventional reduction under otherwise identical conditions. However, the microwave heating mechanism in the ironmaking process was not illustrated in detail in 
these studies because the microwave absorption properties of iron oxides were not available at this moment.

From the beginning of this century, along with the development of techniques for the measurement of microwave absorption properties, research associated with microwave ironmaking and steelmaking became more active. Studies on pig iron production using magnetite ore-coal composite or $\mathrm{Fe}_{3} \mathrm{O}_{4}$ with carbon black and other reducing agents have been reported. ${ }^{47-52}$ Meanwhile, the microwave absorption properties (e.g., permittivity and permeability) of some materials for ironmaking were characterized. ${ }^{53-55}$ Considering the heating characteristics of microwave energy, a new technology entitled "Direct Steelmaking through Microwave and Electric Arc Heating" was successfully developed by a research group led by Jiann-Yang Hwang at Michigan Technological University (MTU). ${ }^{56-59}$ A pilot plant for scale-up of the steelmaking system using this technology was also built, as shown in Fig. 1.1.

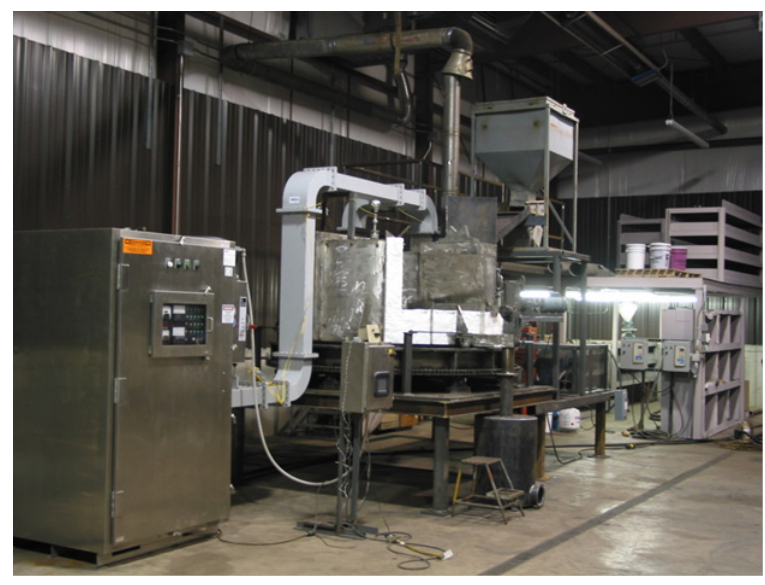

Fig. 1.1. MTU microwave steelmaking rotary hearth system. 
As two of the most common challenges in microwave heating, insufficient microwave absorption and uneven temperature distribution were also observed in microwave assisted steelmaking. Inhomogeneous microwave heating can lead to the formation of hot spot and catastrophic phenomenon of thermal runaway, disturbing the production process and degrading the quality of product. ${ }^{60-64}$ To address these problems for steelmaking using microwaves, it is quite necessary to perform a thorough investigation on the heat transfer in microwave heating. This highly demands the analyses of microwave propagation and dissipation behaviors and characterizations of microwave absorption properties of ironmaking materials. Only if these works have been carried out can microwave heating with high microwave absorption and uniform temperature distribution be achieved.

\subsection{Microwave Heating Fundamentals}

\subsubsection{Permittivity and Permeability}

The following outlines the basic variables related to the interaction between microwaves and materials. For nonmagnetic dielectrics, the absorption of microwaves is related to the permittivity $(\varepsilon)$, which can be defined as follows: ${ }^{65}$

$$
\varepsilon=\varepsilon_{0} \varepsilon_{r}=\varepsilon_{0}\left(\varepsilon_{r}{ }^{\prime}-j \varepsilon_{r}{ }^{\prime \prime}\right)
$$

where $\varepsilon_{0}$ is the permittivity of free space $\left(8.854 \times 10^{-12} \mathrm{~F} \mathrm{~m}^{-1}\right)$, and $j$ is the imaginary unit, $j^{2}=-1$. The complex relative permittivity $\left(\varepsilon_{r}\right)$ is used to describe the constitutive 
relation between the electric flux density and the electric field intensity in lossy dielectrics. It is comprised of two components: the real part of complex relative permittivity or the relative dielectric constant $\left(\varepsilon_{r}{ }^{\prime}\right)$, and the imaginary part of complex relative permittivity or the relative dielectric loss factor $\left(\varepsilon_{r}{ }^{\prime \prime}\right)$. The real part of complex relative permittivity is a measure of the ability of the dielectrics to store electrical energy, while the imaginary part of complex relative permittivity represents the loss of electrical energy in dielectrics. The energy lost from the electric field to the dielectric is eventually converted into thermal energy or heat. Thus, for dielectrics with no magnetic properties, the imaginary part of complex relative permittivity determines the heating rate when microwave energy is applied.

For nonmetallic magnetic materials, such as ferrites, the absorption of microwaves depends on both the permittivity and permeability $(\mu)$. The permeability is defined by the following equation:

$$
\mu=\mu_{0} \mu_{r}=\mu_{0}\left(\mu_{r}{ }^{\prime}-j \mu_{r}{ }^{\prime \prime}\right),
$$

where $\mu_{0}$ is the permeability of free space $\left(4 \pi \times 10^{-7} \mathrm{H} \mathrm{m}^{-1}\right)$, and $\mu_{r}$ is the complex relative permittivity, which is used to describe the constitutive relation between the magnetic flux density and the magnetic field intensity where the magnetic field based loss mechanisms exist. The complex relative permeability is also comprised of two components: the real part of complex relative permeability or the relative magnetic constant $\left(\mu_{r}\right)$, and the imaginary part of complex relative permeability or the relative magnetic loss factor $\left(\mu_{r}{ }^{\prime \prime}\right)$. The real part of complex relative permeability is a measure of 
the ability of the dielectrics to store magnetic energy, while the imaginary part of complex relative permeability represents the loss of magnetic field energy. The energy lost from the magnetic field is again eventually converted into thermal energy or heat. Thus, for magnetic materials, the imaginary part of complex relative permeability heavily influences the heating rate under microwave irradiation.

The ratio of the imaginary to real parts of the permittivity and permeability, define another parameter, the loss tangent $(\tan \delta)$, which is commonly used to indicate the efficiency of conversion of microwave energy into thermal energy within the dielectrics. For nonmagnetic materials, the dielectric loss tangent is defined as

$$
\tan \delta_{\varepsilon}=\varepsilon_{r}{ }^{\prime \prime} / \varepsilon_{r}{ }^{\prime}
$$

Similarly, for magnetic materials, the magnetic loss tangent is defined as follows:

$$
\tan \delta_{\mu}=\mu_{r}{ }^{\prime \prime} / \mu_{r}{ }^{\prime}
$$

\subsubsection{Microwave Penetration Depth}

The main advantage of microwave heating, compared with other conventional methods (e.g., infrared radiation or convective transfer), is that microwaves penetrate into materials, heating volumetrically, and significantly improving the heat transfer to the interior of a sample. ${ }^{66}$ According to the equations for propagation of a TEM electromagnetic wave in materials, a fraction $1-1 / e$ ( that is, $63.2 \%$ ) of the traveling wave energy (or power) is deposited in the material over the distance of a "TEM power 
penetration depth", $D_{p}=1 /(2 \alpha)$, where $\alpha$ is the TEM field attenuation factor. ${ }^{67,68}$ This penetration depth of microwaves is a key parameter in evaluating microwave heating.

In conventional furnaces, heat is transferred by thermal wavelength electromagnetic radiation, and the penetration depth of infrared radiation $\left(f=10^{13} \mathrm{~Hz}\right)$ is very small (much less than $10^{-4} \mathrm{~m}$ ) in the majority of solids. ${ }^{69}$ Thus, only a very thin surface layer of the material will be heated, and heating of the remainder of the material will depend on heat transfer within the material (mainly heat conduction in the material). Since this process requires a relatively long time, rapid heating is difficult to attain in most materials using infrared heating. Conversely, in microwave heating, the penetration depth varies from meters to millimeters because the frequencies used for heating, generally 915 and $2450 \mathrm{MHz}$, are much lower than those used for infrared heating. This means that, for a material that is appropriately sized relative to its penetration depth under microwave irradiation, large surface temperature gradients can be avoided. Relatively uniform rapid heating (volumetric heating) is, therefore, much easier to achieve.

The penetration depth is influenced by both the electric and magnetic properties of nonmetallic materials. ${ }^{70-73}$. Microwave heating investigations have been focused on the nonmagnetic dielectric materials (e.g., ceramics) with limited attention paid to the heating of mixed property materials (e.g., magnetite and other ferrites) under microwave irradiation. ${ }^{73-78}$ However, since magnetic properties can enhance absorption 
of microwaves, some research has been conducted to take advantage of this property. ${ }^{79-}$ ${ }^{83}$ A typical example is microwave assisted steelmaking, where magnetite concentrate is used. ${ }^{56,82,83}$ Magnetite is known as a good absorber of microwaves, with rapid heating and great energy conservation being achieved, ${ }^{56,83}$ but the mechanism is not well quantified, as the high temperature microwave properties have not been measured. Although the determination of the TEM power penetration depth in nonmagnetic materials has been reported, the measurements giving the high-temperature penetration depth of microwaves in materials with non-zero susceptibility (magnetic dielectrics) are still lacking. ${ }^{73,84,85}$ Through determination of the penetration depth, the optimization of dimensions of materials in microwave heating can be achieved, improving the energy efficiency.

Neglecting magnetic effects (i.e., $\mu_{r}^{\prime \prime}=0$ ), the TEM power penetration depth, $D_{p}$, is defined as the distance from the surface into the dielectric at which the traveling wave power drops to $e^{-1}$ from its value at the surface. ${ }^{85}$ It can be expressed as

$$
D_{p}=\frac{\lambda_{0}}{2 \pi\left(2 \varepsilon_{r}{ }^{\prime}\right)^{1 / 2}}\left\{\left[1+\left(\frac{\varepsilon_{r}^{\prime \prime}}{\varepsilon_{r}{ }^{\prime}}\right)^{2}\right]^{1 / 2}-1\right\}^{-1 / 2}
$$

where $\lambda_{0}$ is the microwave wavelength in free space. When the magnetic susceptibility is significant, as in magnetic dielectrics such as ferrites, this equation must be modified. There are several formulations available in the literature, but all of them have not been expressed in simple terms. 


\subsubsection{Microwave Power Absorption}

Microwave energy is transferred to a material through the interaction of the electromagnetic field at the molecular level. For nonmagnetic material, the dielectric properties determine the electromagnetic field in the material. The resistance of the induced motions of free or bound charges and rotation of the dipoles during the interaction of microwaves with the dielectric material due to inertial, elastic, and frictional forces causes losses resulting in volumetric heating. According to this mechanism, the instantaneous power absorbed per unit volume $P$ is expressed as ${ }^{5}$

$$
P=\sigma|E|^{2}=2 \pi f \varepsilon_{0} \varepsilon_{r}^{\prime} \tan \delta|E|^{2}=2 \pi f \varepsilon_{0} \varepsilon_{r}^{\prime \prime}|E|^{2}
$$

where $E$ is the electric field, $\sigma$ is the total effective conductivity, and $f$ is the microwave frequency.

Equation (1.6) indicates that the power absorbed in materials strongly depends on the electromagnetic field $(E$ and $f)$ and the dielectric properties $\left(\varepsilon_{r}{ }^{\prime \prime}\right)$. The understanding of microwave heating is thus based on the analyses of both microwave and materials. However, it should be mentioned that this equation does not consider the thermal contribution from magnetic response in the material under electromagnetic field. Such limitation may cause inaccurate analysis in the microwave heating for magnetic materials. Previous research showed that powered metal compacts were effectively heated in the $H$ field, but not in the $E$ field. Metal-ceramic composites could be heated

up in both microwave $E$ and $H$ fields. ${ }^{86,87}$ The main mechanisms behind the magnetic 
contribution include the hysteresis, eddy currents, magnetic resonance, and domain wall oscillations. ${ }^{88}$ Therefore, the neglect of magnetic component effect in energy transfer from microwaves to materials is no longer acceptable and accurate heat transfer in microwave heating needs the incorporation of the effect of the magnetic fields. This can be achieved by determining the power absorption of materials using Poynting's theorem. ${ }^{89}$

\subsubsection{Microwave Propagation in Materials}

As discussed above, the interaction between microwaves and materials relies on the dielectric and magnetic properties of materials. The materials can then be divided into three types, depending on the microwave propagation behaviors inside, as shown in Table 1.1.90

Table 1.1

Materials classification in microwave heating.

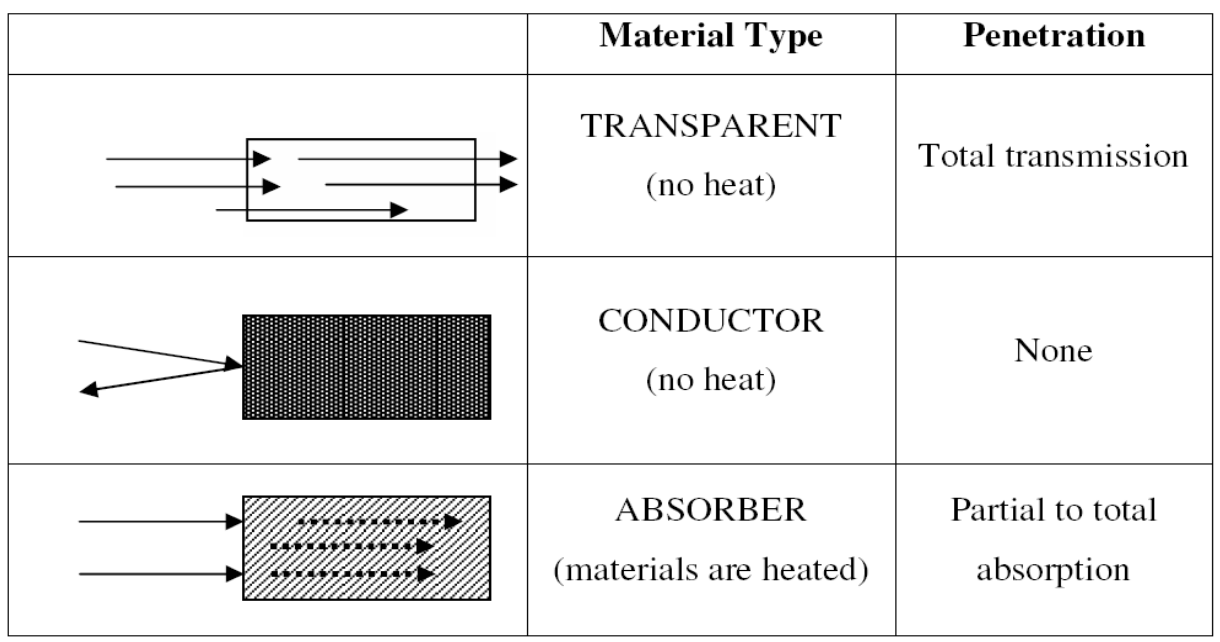


As indicated in Table 1.1, there are three main groups: transparent materials, conductors and absorbers. Total microwave transmission occurs in the transparent materials (no or quite low dielectric and magnetic responses). It indicates that microwaves propagate inside the materials without energy loss, resulting in no thermal energy transfer. In other words, this type of material cannot be efficiently heated in a microwave field. For conductors, they generally reflect microwaves, as demonstrated in a large amount of research. However, it should be emphasized that this is only true for bulk conductors rather than conductor powders.

The microwave absorbers are generally the materials with noticeable dielectric and magnetic losses. This type of material can be heated under microwave irradiation and the degree of microwave heating efficiency is related to the magnitudes of the permittivity and permeability. It can then be divided into several sub-types, for example, lossy dielectric materials and magnetic dielectrics. To gain further understanding of the microwave-material interaction, microwave propagation behaviors in various materials should be characterized. Due to fast microwave velocity in most media, it is almost impossible to visualize the microwave propagation process in experiments. This indicates that modeling and simulation based on the numerical method may provide a solution to this problem. 


\subsubsection{Characterization of Microwave Absorption Properties of Materials}

Microwave heating has been recognized as a technique depending on the properties of materials. Analysis of microwave heating process requires the characterization of the microwave absorption properties of materials. This further demands the measurements of the permittivity and permeability at varying frequencies and temperatures.

Typical methods for permittivity measurement include open-ended coaxial probe method (OECPM), transmission-line method (TLM), and resonant cavity method (RCM). OECPM is quite suitable for liquids and soft solid samples. It is accurate, fast, and broadband (from 0.2 to $20 \mathrm{GHz}$ ) and requires little sample preparation. But the accuracy of this method is not high enough for measuring the materials with low

dielectric properties. ${ }^{91-93}$ TLM belongs to a large group of nonresonant methods of measuring complex permittivity of materials in a microwave range. ${ }^{94}$ There are three main types of transmission lines used as the measurement cell in TLM: rectangular waveguide, coaxial line, and microstrip line. This method generally gives a good accuracy for high-loss materials. It is a time-consuming technique and has rigid requirements on sample shape and sizes. Usually it is more expensive for the same range of frequency than OECPM. ${ }^{95}$ RCM is also widely utilized in measuring the complex permittivity of lossy materials. The most popular resonant cavity method is the perturbation method (also known as the cavity perturbation measurement technique, CPMT). This method is more accurate than the waveguiding methods. It is particularly 
suited for medium-loss and low-loss materials and substances. ${ }^{96-98}$ However, this method generally can only measure the precisely shaped small-sized sample at a fixed frequency. Commercial systems from Hewlett-Packard are also more expensive than the OECPM system, resulting in a high measurement cost.

Considering the accuracy for the measurement, CPMT is employed in this research. This method measures the difference in the microwave cavity response between a cavity with an empty sample-holder and the same cavity with a sample-holder plus the sample, and uses this to calculate either the permittivity or permeability, depending on the field type (electric or magnetic) in the region of the cavity in which the sample is placed. ${ }^{99-102}$ The technique measures the frequency shift and the change in loaded quality factor $(Q)$ of a resonant mode of the cavity caused by inserting a sample.

\section{$\underline{\text { Permittivity Measurements }}$}

The permittivity was measured by placing the sample in the central region of maximum electric field in a $\mathrm{TM}_{0 \mathrm{n} 0}$ cavity (Fig. 1.2), ${ }^{65}$ and measuring the frequency shift and quality factors. The electric susceptibility $\left(\chi_{e}=\chi_{e}{ }^{\prime}-\mathrm{j} \chi_{e}{ }^{\prime \prime}\right)$ can be calculated through the following equation:

$$
\frac{\Delta f}{f_{e}}+j\left(\frac{1}{2 Q_{L, S}}-\frac{1}{2 Q_{L, E}}\right)=\frac{-\chi_{e}}{1+F_{s h} \chi_{e}}\left(\frac{V_{s}}{V_{c}}\right) A,
$$

where, in the present case, $f_{e}$ is the specific cavity mode frequency $(915 \pm 0.5 \% \mathrm{MHz}$ or 
$2450 \pm 0.5 \% \mathrm{MHz}), Q_{L, E}$ is the loaded cavity quality factor with the empty holder, $\Delta f$ is the frequency shift produced by the sample, $Q_{L, S}$ is the loaded cavity $\mathrm{Q}$ with the holder and sample, $F_{s h}$ is a real number dependent only on the sample shape, $V_{s}$ and $V_{c}$ are the respective sample and cavity volumes, $A$ is a real calibration constant dependent only on the shape of the electric fields in the absence of the sample. The two calibration constants in the formula, $A$ and $F_{s h}$ can be determined either by comparison with computer simulations or by comparison with known samples. Solid or liquid calibration samples are used, depending upon whether the sample material is liquid (or a tamped-in powder) or solid (carved or core-drilled sample or pressed pellets). The absolute calibration is normally done at room temperatures, and uses measurements on known high purity materials (similar to ASTM Standard Test Method, D2520-86, Method C). It should be noted that for very large values of electric susceptibility, $\chi_{e}$, the value of the fractional frequency shift approaches an asymptotic value $\left(-A / F_{s h}\right)^{*}\left(V_{s} / V_{c}\right)$. Thus the fractional error in determination of the susceptibility is proportional to the susceptibility.

The measurement system is illustrated in Fig. 1.2. The main system components are a high temperature resistance furnace and a cylindrical $\mathrm{TM}_{0 \mathrm{n} 0}$ cavity with a diameter of $580 \mathrm{~mm}$ and a length of $50 \mathrm{~mm}$. To do a measurement at a specific temperature, the top section of the holder which has the sample in it is raised into the furnace, and held at least $5 \mathrm{~min}$ to ensure that the equilibrium furnace temperature is achieved in the sample. Then the hot sample and holder are rapidly lowered into the central, maximum electric 
field, region of thick-walled, well-cooled copper $\mathrm{TM}_{0 \mathrm{n} 0}$ cavity. The resonant frequencies and loaded quality factor, $Q_{L, S}$, of the cavity modes are rapidly measured by a Hewlett-Packard 8753 network analyzer and stored for off-line analysis (which includes subtraction of hot empty sample holder effects which were previously measured). The sample and holder are typically out of the furnace for $\sim 1.5$ seconds for each frequency measured - in the present case of two frequencies, which means about 3 seconds out of the furnace. The sample temperature decrease during this period is small (typically $<10$ degrees up to $1000{ }^{\circ} \mathrm{C}$ ) and the cooling rates are known and included in the calculation of the exact temperature of a measurement. The permittivity measurements can be done from room temperature in specified steps to the given temperature (up to $1200{ }^{\circ} \mathrm{C}$ ), and then cooled down.

After the completion of the sequence of measurements, the mass and dimension of the sample are remeasured. The whole measurement sequence is preprogrammed and computer-controlled over the desired set of temperatures, with furnace, linear motion actuator (sample movement), and network analyzer all controlled by a PC running Labview control software.

\section{Permeability Measurements}

The complex permeability was measured using a ridge-loaded-WR284 waveguidebased resonant cavity which produces a region of maximum magnetic field strength at 
the shorted end of the waveguide. ${ }^{102}$ The sample was moved rapidly into this maximum field, and again the frequency shift and change in loaded $Q$ are measured. The complex permeability is calculated using a formula identical in form to eq. (1.7), but with the appropriate frequency and $Q$ and shape factors and calibration constants for the magnetic cavity.

The permeability measurements are, in practice, less accurate than permittivity measurements because large corrections have to be made for the frequency and $Q$ shift in the magnetic cavity that are caused by the small, but significant, electric field that exists in the high magnetic field region. Since the complex dielectric constant is almost always much larger (typically 10 times) than the complex permeability, the small electric field causes frequency shifts and $Q$ changes that must be taken into account. Thus the magnetic measurements require subtractions that depend upon reliable values at each temperature of the complex permittivity. Only after these corrections have been made, can the complex magnetic susceptibility $\chi_{m}$, be determined by using eq. (1.8):

$$
\frac{\Delta f}{f_{m}}+j\left(\frac{1}{2 Q_{L, S}}-\frac{1}{2 Q_{L, E}}\right)=\frac{-\chi_{m}}{1+F_{s h} \chi_{m}}\left(\frac{V_{s}}{V_{c}}\right) A,
$$

where $f_{m}$ is the cavity mode frequency in the permeability test, and $\Delta f$ and $Q$ are only from the magnetic field induced contribution. Even after these corrections, other problems exist in the interpretation of the apparent measured magnetic susceptibility. If the sample has a moderately large high-frequency electrical conductivity (which is determined by the permittivity measurements), the time-varying microwave magnetic 
field can induce electrical fields in the sample that produce azimuthal induced currents in the sample, completely analogous to low frequency induction heating. This represents another mechanism of power loss in the sample, and produces power loss and a reduction in $Q$. However, these losses are mainly due to the electrical conductivity of the sample, which is a part of the imaginary permittivity. These "induced electric field" losses are not significant in the permittivity measurements because of the very low magnetic field in the central region of the $\mathrm{TM}_{0 \mathrm{n} 0}$ cavity, relative to the electric field.

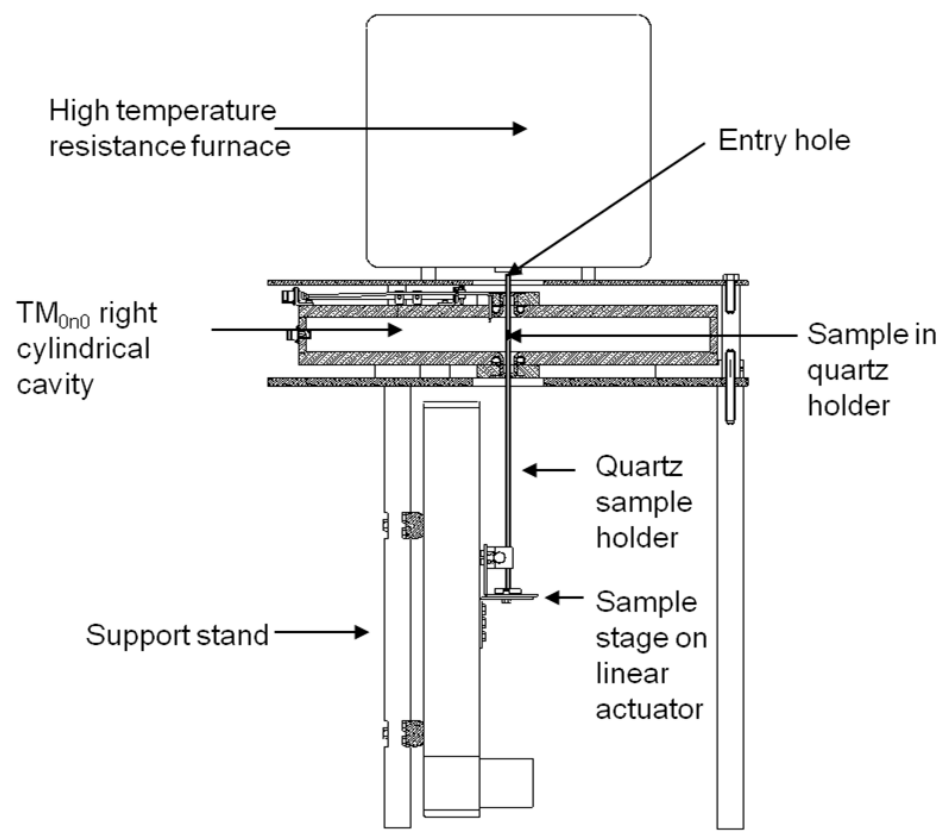

Fig. 1.2. Schematic of the $T M_{0 n 0}$ cavity system (in cross-section) showing the linear actuator with the quartz sample holder and a sample located on axis in the center of the cavity.

Another problem occurs if the sample conductivity is very high, as with an almost metallic sample. If the conductivity is such that the skin depth is small relative to the 
diameter of the sample, this implies that the induced currents produce a "bucking" magnetic field in the sample interior that exactly counters the cavity magnetic field. In this way, all magnetic fields are excluded from the interior of the sample. This is the typical Lenz's law situation, and implies an "apparent" magnetic susceptibility, $\chi_{m}=-1$, or a value $\mu_{r}=0$ if interpreted incorrectly using eq. (1.8). These problems can only be avoided by a detailed knowledge of the physical and solid state properties of the sample at all temperatures.

\subsection{Heat Transfer Analysis of Microwave Heating}

Although microwave has shown its superiority in materials heating, a major drawback known as the nonuniform temperature distribution inside materials has also been observed by many researchers. ${ }^{103,104}$ To solve this problem, the accurate temperature determination inside the materials under microwave irradiation is quite necessary. However, an exact temperature measurement in microwave heating has been identified as a hard work since most common temperature measurement tools like thermocouple and pyrometer may not provide precise measurement data. The interaction between thermocouple and microwave lowers the measurement accuracy while the complexity of emissivity considerations required to properly apply optical pyrometry heavily limits its extensive application. ${ }^{105}$ 
In comparison with the direct temperature measurement, temperature prediction by analytical and numerical methods seems to offer a promising solution to this problem. Both analytical and numerical methods are required to solve the heat transfer differential equation coupled with Maxwell's equations, but the former is found to be much more difficult since the heat generation from microwave heating and complex boundary conditions including convective and radiative heat transfer need to be considered simultaneously to obtain a closed-form mathematical solution. ${ }^{106-108}$ Conversely, numerical modeling has been proved as an efficient and accurate method to predict the temperature of materials during microwave heating in the past 20 years. ${ }^{109-}$ ${ }^{113}$ Most of those studies have been focused on the utilization of microwave in the field of food processing where only heat diffusion and/or convection were considered. Meanwhile, the variations of dielectric properties of materials during the heating were generally ignored due to a relatively low temperature range investigated (generally < $120^{\circ} \mathrm{C}$ ). It is obvious that the same assumption cannot be applied at high temperatures where heat radiation becomes quite strong and the dielectric properties may change dramatically. Therefore, to accurately simulate heat transfer in high-temperature microwave processing of materials, the radiation effect and the temperature dependency of dielectric properties of materials have to be considered. 


\section{Chapter 2 Goals and Hypotheses}

The main objective of this research is to investigate the heating fundamentals for achieving highly efficient microwave assisted steelmaking. The specific goals include:

(1) Derive new equations for characterizing microwave decay.

(2) Identify the contribution of magnetic loss to microwave heating.

(3) Characterize microwave absorption properties of materials for ironmaking.

(4) Model microwave propagation in dielectric media.

(5) Simulate heat transfer in microwave heating.

(6) Improve microwave absorption and heating uniformity.

\subsection{Derive New Equations for Characterizing Microwave Decay}

As mentioned previously, one of key parameters in microwave heating is the microwave penetration depth $\left(D_{p}\right)$. The conventional equation for $D_{p}$ does not include the contribution of permeability, which is quite important in the microwave heating of various materials including powdered metal, semiconductors, magnetic dielectrics, etc. Thus, we suppose that a complete and simplified equation for determining the transverse electromagnetic mode (TEM) power penetration depth of microwaves in materials having both magnetic and dielectric responses is necessary and useful for describing the behaviors of microwave propagation and dissipation. Because two other parameters, field attenuation length $\left(D_{f}\right)$ and half-power depth $\left(D_{h}\right)$, are also employed 
to indicate the microwave dissipation in materials in microwave heating, the corresponding equations for determining these parameters also deserve attention and need to be developed, too.

\subsection{Identify the Contribution of Magnetic Loss to Microwave Heating}

It is expected that magnetic response contributes to the increase in temperature of materials in microwave heating. This can be indicated by the comparison between microwave heating of magnetite and that of hematite. With magnetic response, the former can be heated much faster than the later under the same experimental conditions. The present work seeks to determine the contribution of magnetic loss to microwave heating through the equation derived for magnetic loss in magnetic dielectric materials.

\subsection{Investigate Microwave Absorption Properties of Materials for Ironmaking}

The above discussion shows that microwave heating strongly depends on the microwave absorption properties of materials. However, so far, no systematic work has been conducted to study these properties of materials for ironmaking. In this study, the dielectric and magnetic properties of hematite (nonmagnetic), magnetite concentrate (magnetic), ferrous oxide (nonmagnetic) and coal (organic) will be measured using the cavity perturbation measurement technique (CPMT). Various techniques, such as X-ray 
diffraction (XRD), field emission-scanning electron microscopy (FE-SEM), high temperature X-ray diffraction (HT-XRD), thermal gravimetric analysis (TGA), and Fourier transform infrared spectroscopy (FTIR), will be employed to help characterize the microwave absorption properties of the materials. Microwave penetration depths and dielectric losses as well as magnetic losses for these materials will be calculated based on the measured values of permittivity and permeability.

\subsection{Model Microwave Propagation in Dielectric Media}

The purpose of this part of research is to further the understanding of microwave propagation and dissipation in various media through the simulation using finitedifference time-domain (FDTD) method. The FDTD method is a popular computational electrodynamics modeling technique, where the time-dependent Maxwell's equations in the partial differential form are discretized using central-difference approximations to the space and time partial derivatives. The resulting finite-difference equations are solved in either software or hardware in a leapfrog manner. ${ }^{114-119}$ The typical packages include CFDTD (PSU version, Pennsylvania) ${ }^{120}$ and Concerto 7.0 (Vector Fields, U.K.). ${ }^{121-123}$ These packages perform the simulation quickly while the corresponding computational process cannot be visualized. To guarantee the simulation results, instead of using these available packages, a specific computational code will be developed using Mathematica 7.0 (Wolfram Research of Champaign, Illinois) in this part of 
research. The simulation patterns for various materials under microwave irradiations will be obtained and analyzed.

\subsection{Simulate Heat Transfer in Microwave Heating}

Actually, the accurate simulation of heat transfer process in microwave heating requires the solution of the equation where the heat diffusion equation is coupled with Maxwell's equations. This needs to consider both the vibrations of electric and magnetic fields in the computation and the standing wave effect inside the materials, which will significantly enhance the computation cost in the simulation. To save the computation power, Lambert's law rather than Maxwell's equations will be applied in the computation. This treatment has been shown as an acceptable technique in the simulation of large-scale microwave heating of materials. ${ }^{124}$ The corresponding codes will be developed using Mathematica 7.0. The effects of various parameters including heating time, microwave power, microwave frequency, and object dimension on the heat transfer in microwave heating of magnetite will be investigated.

\subsection{Improve Microwave Absorption and Heating Uniformity}

Due to the relatively larger penetration depth of microwaves in materials at commonly used frequencies, 915 and $2450 \mathrm{MHz}$, it can be assumed that microwave absorption of materials closely relies on the sample dimension. An appropriate size of the material is 
important for increasing microwave heating efficiency. Furthermore, because the rate of heat conduction in materials is much slower than the oscillation speed of microwave electric and magnetic fields, heating nonuniformity is always observed in experiments. Heat transfer in microwave heating becomes more important in large-scale microwave heating. In this section of the research, we suppose that the dimensions of materials subjected to microwave heating can be optimized to increase microwave absorption and to improve heating uniformity. Reflection loss and impedance matching will be introduced to achieve this aim. 


\title{
Chapter 3 New Equations for Characterizing Microwave
}

\author{
Decay $^{*}$
}

\subsection{Derivation of Microwave Power Penetration Depth Equation}

In a homogeneous medium and without convection or external currents, the differential Maxwell's equations in lossy dielectrics are given by

$$
\begin{gathered}
\nabla \times E=-j \omega \mu H, \\
\nabla \times H=j \omega \varepsilon E, \\
\nabla \cdot E=0, \\
\nabla \cdot H=0,
\end{gathered}
$$

\footnotetext{
* The content of this chapter was previously published in ISIJ International ${ }^{65}$ by Zhiwei Peng, Jiann-Yang Hwang, Joe Mouris, Ron Hutcheon, Xiaodi Huang and in TMS Annual Meeting, 2nd International Symposium on High-Temperature Metallurgical Processing - Held During the TMS 2011 Annual Meeting and Exhibition ${ }^{125}$ by Zhiwei Peng, Jiann-Yang Hwang, Xiaodi Huang, Matthew Andriese, Wayne Bell.

Reproduced with permission from ISIJ International: Zhiwei Peng, Jiann-Yang Hwang, Joe Mouris, Ron Hutcheon, Xiaodi Huang. Microwave Penetration Depth in Materials with Non-zero Magnetic Susceptibility. ISIJ International. 2010;50(11):1590-1596. Copyright (C) 2010 The Iron and Steel Institute of Japan. (See Appendix A-1 for copyright permission).

Reproduced with permission from TMS Annual Meeting, 2nd International Symposium on HighTemperature Metallurgical Processing - Held During the TMS 2011 Annual Meeting and Exhibition: Zhiwei Peng, Jiann-Yang Hwang, Xiaodi Huang, Matthew Andriese, Wayne Bell. Microwave Field Attenuation Length and Half-power Depth in Magnetic Materials. TMS Annual Meeting, 2nd International Symposium on High-Temperature Metallurgical Processing - Held During the TMS 2011 Annual Meeting and Exhibition. 2011;51-57. Copyright (C) 2011 The Minerals, Metals and Materials Society. (See Appendix A-2 for copyright permission).
} 
where $E$ is the electric field intensity, $H$ is the magnetic field intensity and $\omega$ is the angular frequency of microwaves. Considering vector multiplication, for Maxwell's equations, we have

$$
\begin{aligned}
& \nabla \times \nabla \times E=\nabla(\nabla \cdot E)-\nabla^{2} E=-j \omega \mu \nabla \times H=\omega^{2} \mu \varepsilon E=-\gamma^{2} E, \\
& \nabla \times \nabla \times H=\nabla(\nabla \cdot H)-\nabla^{2} H=j \omega \varepsilon \nabla \times E=\omega^{2} \mu \varepsilon H=-\gamma^{2} H,
\end{aligned}
$$

where $\gamma$ is the propagation constant. Thus, we obtain

$$
\begin{aligned}
& \nabla^{2} E-\gamma^{2} E=0, \\
& \nabla^{2} H-\gamma^{2} H=0 .
\end{aligned}
$$

The two equations above are also known as the Helmholtz equations. Assuming a TEM plane wave traveling in the $z$ direction with the $x$ component of electric field intensity, the Helmholtz equations would yield

$$
\frac{d^{2} E_{x}}{d z^{2}}-\gamma^{2} E_{x}=0
$$

The general solution to this wave equation is

$$
E_{x}(z)=C_{1} \mathrm{e}^{-\gamma z}+C_{2} \mathrm{e}^{+\gamma z}=C_{1} \mathrm{e}^{-\alpha z} \mathrm{e}^{-j \beta z}+C_{2} \mathrm{e}^{\alpha z} \mathrm{e}^{j \beta z},
$$

where $C_{1}$ and $C_{2}$ are constants. In time domain, the representation of $E$ is

$$
\begin{gathered}
E_{x}(z, t)=\operatorname{Re}\left\{E_{x}(z) \mathrm{e}^{j \omega t}\right\}, \\
E_{x}(z, t)=C_{1} \mathrm{e}^{-\alpha z} \cos (\omega t-\beta z)+C_{2} \mathrm{e}^{\alpha z} \cos (\omega t+\beta z) .
\end{gathered}
$$

Here, the propagation constant $(\gamma)$ is a complex number and can be expressed as

$$
\gamma=\alpha+j \beta,
$$


where $\alpha$ is the field attenuation factor and $\beta$ is the phase constant. From the definition, we have

$$
\begin{gathered}
\gamma=j \omega(\mu \varepsilon)^{1 / 2}, \\
\gamma=j \omega\left(\mu_{0} \mu_{r} \varepsilon_{0} \varepsilon_{r}\right)^{1 / 2}, \\
\gamma=j \omega\left[\mu_{0}\left(\mu_{r}{ }^{\prime}-j \mu_{r}{ }^{\prime \prime}\right) \varepsilon_{0}\left(\varepsilon_{r}{ }^{\prime}-j \varepsilon_{r}{ }^{\prime \prime}\right)\right]^{1 / 2}, \\
\gamma=\left[-\omega^{2} \varepsilon_{0} \mu_{0}\left(\varepsilon_{r}{ }^{\prime} \mu_{r}{ }^{\prime}-\varepsilon_{r}{ }^{\prime \prime} \mu_{r}{ }^{\prime \prime}\right)+j \omega^{2} \varepsilon_{0} \mu_{0}\left(\varepsilon_{r}{ }^{\prime} \mu_{r}{ }^{\prime \prime}+\varepsilon_{r}{ }^{\prime \prime} \mu_{r}{ }^{\prime}\right)\right]^{1 / 2} .
\end{gathered}
$$

By separating the real and imaginary parts of eq. (3.17) and equating the real part with $\alpha$, we have

$$
\begin{aligned}
& \alpha=\omega\left(\frac{\varepsilon_{0} \mu_{0}}{2}\right)^{1 / 2}\left\{\varepsilon_{r}{ }^{\prime \prime} \mu_{r}{ }^{\prime \prime}-\varepsilon_{r}{ }^{\prime} \mu_{r}{ }^{\prime}+\left[\begin{array}{l}
\left(\varepsilon_{r}{ }^{\prime} \mu_{r}{ }^{\prime}\right)^{2}+\left(\varepsilon_{r}{ }^{\prime} \mu_{r}{ }^{\prime \prime}\right)^{2}+ \\
\left(\varepsilon_{r}{ }^{\prime} \mu_{r}{ }^{\prime}\right)^{2}+\left(\mu_{r}{ }^{\prime} \varepsilon_{r}{ }^{\prime \prime}\right)^{2}
\end{array}\right]^{1 / 2}\right\}^{1 / 2}, \\
& \alpha=2 \pi f\left(\frac{\varepsilon_{0} \mu_{0}}{2}\right)^{1 / 2}\left\{\varepsilon_{r}{ }^{\prime \prime} \mu_{r}{ }^{\prime \prime}-\varepsilon_{r}{ }^{\prime} \mu_{r}{ }^{\prime}+\left[\begin{array}{l}
\left(\varepsilon_{r}{ }^{\prime} \mu_{r}{ }^{\prime}\right)^{2}+\left(\varepsilon_{r}{ }^{\prime \prime} \mu_{r}{ }^{\prime \prime}\right)^{2}+ \\
\left(\varepsilon_{r}{ }^{\prime} \mu_{r}{ }^{\prime \prime}\right)^{2}+\left(\mu_{r}{ }^{\prime} \varepsilon_{r}{ }^{\prime \prime}\right)^{2}
\end{array}\right]^{1 / 2}\right\}^{1 / 2}, \\
& \alpha=\frac{\sqrt{2} \pi}{\lambda_{0}}\left\{\varepsilon_{r}{ }^{\prime \prime} \mu_{r}{ }^{\prime \prime}-\varepsilon_{r}{ }^{\prime} \mu_{r}{ }^{\prime}+\left[\begin{array}{l}
\left(\varepsilon_{r}{ }^{\prime} \mu_{r}{ }^{\prime}\right)^{2}+\left(\varepsilon_{r}{ }^{\prime} \mu_{r}{ }^{\prime \prime}\right)^{2}+ \\
\left(\varepsilon_{r}{ }^{\prime} \mu_{r}{ }^{\prime \prime}\right)^{2}+\left(\mu_{r}{ }^{\prime} \varepsilon_{r}{ }^{\prime \prime}\right)^{2}
\end{array}\right]^{1 / 2}\right\}^{1 / 2},
\end{aligned}
$$

The wave is attenuated as it traverses the medium and therefore the power is dissipated. According to the definition of the (1/e) power penetration depth, we obtain

$$
D_{p}=\frac{1}{2 \alpha},
$$




$$
D_{p}=\frac{\lambda_{0}}{2 \sqrt{2} \pi}\left\{\varepsilon_{r}{ }^{\prime \prime} \mu_{r}{ }^{\prime \prime}-\varepsilon_{r}{ }^{\prime} \mu_{r}{ }^{\prime}+\left[\begin{array}{l}
\left(\varepsilon_{r}{ }^{\prime} \mu_{r}{ }^{\prime}\right)^{2}+\left(\varepsilon_{r}{ }^{\prime \prime} \mu_{r}{ }^{\prime \prime}\right)^{2}+ \\
\left(\varepsilon_{r}{ }^{\prime} \mu_{r}{ }^{\prime \prime}\right)^{2}+\left(\mu_{r}{ }^{\prime} \varepsilon_{r}{ }^{\prime \prime}\right)^{2}
\end{array}\right]^{1 / 2}\right\}^{-1 / 2},
$$

or, in terms of loss tangent,

$$
D_{p}=\frac{\lambda_{0}}{2 \pi\left(2 \varepsilon_{r}{ }^{\prime} \mu_{r}^{\prime}\right)^{1 / 2}}\left[\begin{array}{l}
\left(1+\tan ^{2} \delta_{\varepsilon} \tan ^{2} \delta_{\mu}+\tan ^{2} \delta_{\mu}+\tan ^{2} \delta_{\varepsilon}\right)^{1 / 2} \\
+\tan \delta_{\varepsilon} \tan \delta_{\mu}-1
\end{array}\right]^{-1 / 2} .
$$

Equation (3.22) is, therefore, the equation for the determination of microwave penetration depth of magnetic dielectrics. Assuming $\mu_{r}{ }^{\prime \prime}=0$, eq. (3.22) simplifies to

$$
D_{p}=\frac{\lambda_{0}}{2 \pi\left(2 \varepsilon_{r}\right)^{1 / 2}}\left[\left(1+\left(\frac{\varepsilon_{r}^{\prime \prime}}{\varepsilon_{r}^{\prime}}\right)^{2}\right)^{1 / 2}-1\right]^{-1 / 2}
$$

which is the same as eq. (1.5). For magnetic dielectrics, eq. (3.22) should be applied in the calculation of the power penetration depth.

\subsection{Derivation of Field Attenuation Length Equation}

According to the definition of the field attenuation length (skin depth), $D_{f}$, we obtain

$$
D_{f}=\frac{1}{\alpha}=\frac{\lambda_{0}}{\sqrt{2} \pi}\left\{\varepsilon_{r}{ }^{\prime} \mu_{r}{ }^{\prime \prime}-\varepsilon_{r}{ }^{\prime} \mu_{r}{ }^{\prime}+\left[\begin{array}{l}
\left(\varepsilon_{r}{ }^{\prime} \mu_{r}{ }^{\prime}\right)^{2}+\left(\varepsilon_{r}{ }^{\prime} \mu_{r}{ }^{\prime \prime}\right)^{2}+ \\
\left(\varepsilon_{r}{ }^{\prime} \mu_{r}{ }^{\prime}\right)^{2}+\left(\mu_{r}{ }^{\prime} \varepsilon_{r}{ }^{\prime \prime}\right)^{2}
\end{array}\right]^{1 / 2}\right\}^{-1 / 2},
$$

or, in terms of loss tangent, 


$$
D_{f}=\frac{\lambda_{0}}{\pi\left(2 \varepsilon_{r}{ }^{\prime} \mu_{r}{ }^{\prime}\right)^{1 / 2}}\left[\begin{array}{l}
\left(1+\tan ^{2} \delta_{\varepsilon} \tan ^{2} \delta_{\mu}+\tan ^{2} \delta_{\mu}+\tan ^{2} \delta_{\varepsilon}\right)^{1 / 2} \\
+\tan \delta_{\varepsilon} \tan \delta_{\mu}-1
\end{array}\right]^{-1 / 2},
$$

where $\tan \delta_{\varepsilon}$ and $\tan \delta_{\mu}$ are dielectric loss tangent and magnetic loss tangent, respectively.

\subsection{Derivation of Half-power Depth Equation}

For the microwave half-power depth, $D_{h}$, it is defined as the distance from the surface into the dielectric at which the traveling wave power drops to $1 / 2$ from its value at the surface. From this definition, we have

$$
\begin{aligned}
& D_{h}=\frac{\ln (2)}{2 \alpha}, \\
& D_{h}=\frac{\ln (2) \lambda_{0}}{2 \sqrt{2} \pi}\left\{\varepsilon_{r}{ }^{\prime \prime} \mu_{r}{ }^{\prime \prime}-\varepsilon_{r}{ }^{\prime} \mu_{r}{ }^{\prime}+\left[\begin{array}{l}
\left(\varepsilon_{r}{ }^{\prime} \mu_{r}{ }^{\prime}\right)^{2}+\left(\varepsilon_{r}{ }^{\prime \prime} \mu_{r}{ }^{\prime \prime}\right)^{2}+ \\
\left(\varepsilon_{r}{ }^{\prime} \mu_{r}{ }^{\prime}\right)^{2}+\left(\mu_{r}{ }^{\prime} \varepsilon_{r}{ }^{\prime \prime}\right)^{2}
\end{array}\right]^{1 / 2}\right\}^{-1 / 2},
\end{aligned}
$$

or, in terms of loss tangent,

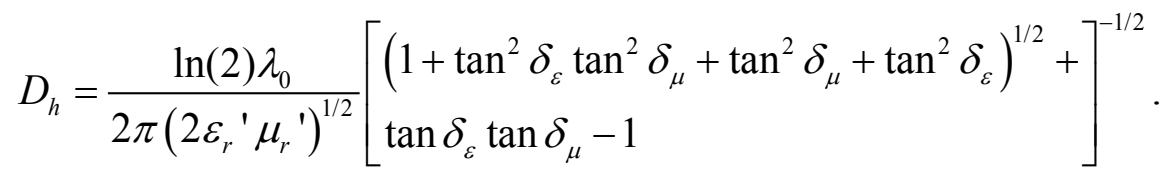




\section{Chapter 4 Magnetic Loss in Microwave Heating ${ }^{\dagger}$}

\subsection{Magnetic Loss and Heat Generation}

As aforementioned, microwave heating has gained exceptionally broad applications in the field of material synthesis and processing. The understanding of physical mechanism for this technique further advances these utilizations. Among the various intriguing aspects of microwave heating, one point receiving the increasing attention is the superior advantage of microwave magnetic field heating over electric field heating for materials including magnetic dielectrics and metal powders. The different types of heating may generate a distinguished difference between microwave magnetic loss and dielectric loss. ${ }^{86}$ The relative experimental phenomenon was first observed in the heating of various metal powders and magnetic as well as nonmagnetic ceramics by using separated microwave electric and magnetic fields. ${ }^{86,88,127}$ It was found that microwave magnetic field heating achieves a much faster temperature increase than electric field heating for magnetic dielectric materials (e.g., ferrites) and certain conductive powder materials (e.g., cobalt). The similar results and observations were then reported and confirmed by other studies. ${ }^{128-130}$ These experimental studies clearly

\footnotetext{
$\dagger$ The content of this chapter was previously published in Applied Physics Express ${ }^{126}$ by Zhiwei Peng, Jiann-Yang Hwang, Matthew Andriese.

Reproduced with permission from Applied Physics Express: Zhiwei Peng, Jiann-Yang Hwang, Matthew Andriese. Magnetic Loss in Microwave Heating. Applied Physics Express. 2012;5(2):027304-027304-3. Copyright ( 2012 The Japan Society of Applied Physics. (See Appendix B-1 for copyright permission).
} 
show that magnetic loss contributes significantly to the microwave heating and deserves further investigations, especially on the theoretical aspect.

In the past few years, several theoretical works were carried out to explore magnetic loss mechanism. Among them, the selective heating mechanism of magnetic metal oxides by a microwave magnetic field using dissipative spin dynamics simulation was reported. It was claimed that the heating occurs due to the response of magnetization to microwaves, originating from electron spins. ${ }^{131}$ More recently, the effect of magnetic anisotropy on magnetic loss in microwave magnetic field heating of ferromagnetic materials was identified. ${ }^{132}$ These studies, however, generally focused on several specific materials and did not quantify the magnetic loss from a theoretical viewpoint.

The quantification of magnetic loss is essential for clarifying the microwave heating mechanism and analyzing heat transfer in materials under microwave irradiation. This is attributed to the fact that temperature increase in materials depends on the heat generation during microwave processing, which includes the contributions from dielectric loss and magnetic loss.

The work in this chapter seeks to determine the contribution of magnetic loss to microwave heating through the equation derived for magnetic loss in magnetic dielectric materials. It was started by the derivation of the equation for microwave power dissipation in media. The difference between dielectric loss and magnetic loss 
was subsequently evaluated for five ferrites, namely $\mathrm{BaFe}_{12} \mathrm{O}_{19}, \mathrm{SrFe}_{12} \mathrm{O}_{19}, \mathrm{CuFe}_{2} \mathrm{O}_{4}$, $\mathrm{CuZnFe}_{4} \mathrm{O}_{4}$ and $\mathrm{NiZnFe}_{4} \mathrm{O}_{4}$. It is demonstrated that magnetic loss contributes greater to microwave heating of magnetic dielectrics than dielectric loss, which agrees well with the observation in previous experimental work. From the calculations, it is believed that the formula developed in this work can be used to study the heating characteristics of materials in the microwave magnetic field and to analyze the heat transfer process in microwave heating of materials.

\subsection{Derivation of Microwave Power Dissipation Equation}

The power flows through a closed surface can be calculated from the integration of the Poynting vector $S$ :

$$
S=E \times H .
$$

The Average Power is

$$
P_{a v}=\int_{s^{\prime}}\langle S\rangle d s^{\prime}=-\frac{1}{2} \int_{s^{\prime}} \operatorname{Real}\left(E \times H^{*}\right) \cdot d s^{\prime} .
$$

The deduction process is as follows:

$$
\begin{gathered}
\nabla \times H=j \omega \varepsilon E+J, \\
\nabla \times H=j \omega \varepsilon_{0} \varepsilon_{r, d} E+\sigma E, \\
\nabla \times H=\left(\omega \varepsilon_{0} \varepsilon_{r, d}{ }^{\prime \prime}+j \omega \varepsilon_{0} \varepsilon_{r, d}^{\prime}\right) E+\sigma E,
\end{gathered}
$$




$$
\begin{gathered}
\nabla \times H=\omega \varepsilon_{0}\left(\varepsilon_{r, d}{ }^{\prime}+\frac{\sigma}{\omega \varepsilon_{0}}\right) E+j \omega \varepsilon_{0} \varepsilon_{r, d}{ }^{\prime} E, \\
\nabla \times H=\omega \varepsilon_{0} \varepsilon_{r}{ }^{\prime} E+j \omega \varepsilon_{0} \varepsilon_{r}{ }^{\prime} E .
\end{gathered}
$$

From mathematics, we have

$$
\begin{gathered}
\nabla \cdot\left(E \times H^{*}\right)=(\nabla \times E) \cdot H^{*}-\left(\nabla \times H^{*}\right) \cdot E, \\
\nabla \cdot\left(E \times H^{*}\right)=\left[\left(-\omega \mu_{0} \mu_{r}{ }^{\prime \prime}-j \omega \mu_{0} \mu_{r}{ }^{\prime}\right) H\right] \cdot H^{*}-\left(\omega \varepsilon_{0} \varepsilon_{r}{ }^{\prime \prime} E-j \omega \varepsilon_{0} \varepsilon_{r}{ }^{\prime} E\right) \cdot E, \\
\nabla \cdot\left(E \times H^{*}\right)=-\omega \mu_{0} \mu_{r}{ }^{\prime \prime} H \cdot H^{*}-\omega \varepsilon_{0} \varepsilon_{r}{ }^{\prime \prime} E \cdot E^{*}-j \omega \mu_{0} \mu_{r}{ }^{\prime} H \cdot H^{*}+j \omega \varepsilon_{0} \varepsilon_{r}{ }^{\prime} E \cdot E^{*} .
\end{gathered}
$$

By using the divergence theorem, we have

$$
\begin{gathered}
\int_{V} \nabla \cdot\left(E \times H^{*}\right) d V=\int_{s^{\prime}}\left(E \times H^{*}\right) d s^{\prime}, \\
\int_{V} \nabla \cdot\left(E \times H^{*}\right) d V=\int_{V}\left(-\omega \mu_{0} \mu_{r}{ }^{\prime} H \cdot H^{*}-\omega \varepsilon_{0} \varepsilon_{r} " E \cdot E^{*}\right) d V \\
-j \omega \int_{V}\left(\mu_{0} \mu_{r}^{\prime} H \cdot H^{*}-\varepsilon_{0} \varepsilon_{r}{ }^{\prime} E \cdot E^{*}\right) d V .
\end{gathered}
$$

Thus,

$$
\begin{gathered}
P_{a v}=\int_{s^{\prime}}\langle S\rangle d s^{\prime}=-\frac{1}{2} \int_{s^{\prime}} \operatorname{Real}\left(E \times H^{*}\right) \cdot d s^{\prime}, \\
P_{a v}=\frac{1}{2} \omega \varepsilon_{0} \varepsilon_{r} " \int_{V}\left(E \cdot E^{*}\right) \cdot d V+\frac{1}{2} \omega \mu_{0} \mu_{r} " \int_{V}\left(H \cdot H^{*}\right) \cdot d V, \\
P_{a v}=\frac{1}{2} \omega \varepsilon_{0} \varepsilon_{r} " E^{2} V+\frac{1}{2} \omega \mu_{0} \mu_{r} " H^{2} V .
\end{gathered}
$$

As indicated in eq. (4.15), microwave heating is dependent on the microwave frequency, dielectric loss factor of the material, magnetic loss factor of the material, microwave electric field strength, and magnetic field strength. 


\subsection{Derivation of Magnetic Loss Equation}

Magnetic loss in a medium during microwave heating relies on the distribution of an electromagnetic field, which is governed by Maxwell's equations. Assuming the microwaves are uniform plane waves incident from air to magnetic dielectric interface propagating in the $z$ direction (Fig. 4.1), then the simplified equation for electric field (E) of the uniform plane wave developed from Maxwell's equations can be described by eq. $(3.9)^{65,133}$

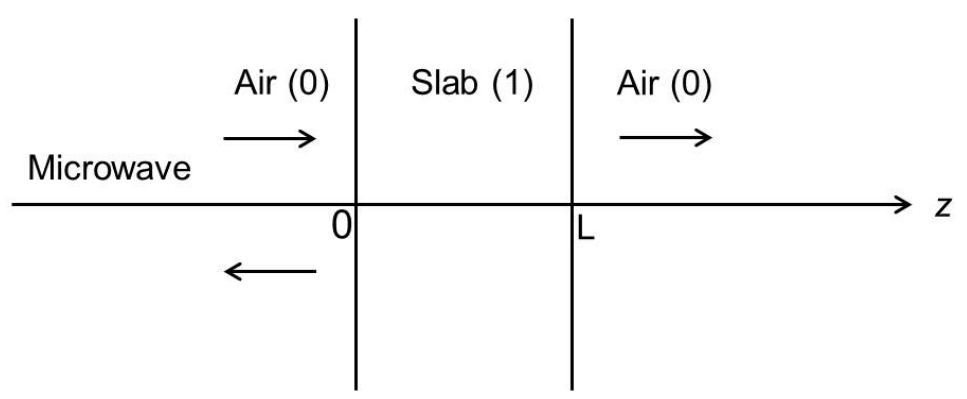

Fig. 4.1. A magnetic dielectric layer subjected to microwaves from the left side.

As shown before, the propagation constant, $\gamma$, can be given as eq. (3.14) with consideration of both permittivity and permeability. It can also be expressed as a complex number with two parts: $\alpha$ and $j \beta$. The simplified form for $\alpha$ is described by eq. (3.20) while the phase constant $\beta$ can be calculated and expressed as

$$
\beta=\frac{\sqrt{2} \pi}{\lambda_{0}}\left\{\varepsilon_{r}{ }^{\prime} \mu_{r}{ }^{\prime}-\varepsilon_{r}{ }^{\prime \prime} \mu_{r}{ }^{\prime \prime}+\left[\begin{array}{l}
\left(\varepsilon_{r}{ }^{\prime} \mu_{r}^{\prime}\right)^{2}+\left(\varepsilon_{r}{ }^{\prime \prime} \mu_{r}{ }^{\prime \prime}\right)^{2}+ \\
\left(\varepsilon_{r}{ }^{\prime} \mu_{r}{ }^{\prime \prime}\right)^{2}+\left(\mu_{r}{ }^{\prime} \varepsilon_{r}{ }^{\prime \prime}\right)^{2}
\end{array}\right]^{1 / 2}\right\}^{1 / 2} .
$$


For the microwave incident from the left side the boundary conditions (at $z=0, L$ ) for the plane wave propagating in the $z$ direction can be simplified as

$$
\begin{gathered}
E_{x, 0}=E_{x, 1}, \\
H_{y, 0}=H_{y, 1},
\end{gathered}
$$

where the subscripts 0 and 1 denote the free space and magnetic dielectric domain, respectively; $H_{y}$ is the magnetic field component of plane wave, which can be determined by using the following formula:

$$
\frac{d E_{x}}{d z}=j \mu \omega H_{y}
$$

The general solution of the plane wave can be obtained as

$$
\begin{gathered}
E_{x}=A e^{-\gamma z}+B e^{\gamma z}, \\
H_{y}=\frac{1}{\eta}\left(A e^{-\gamma z}-B e^{\gamma z}\right),
\end{gathered}
$$

where $\eta$ is the impedance and given as

$$
\eta=j \mu \omega / \gamma
$$

With consideration of incident electric field $\left(E_{0}\right)$, the electric and magnetic fields within magnetic dielectrics $(0 \leq z \leq L)$ can be determined using the following equations:

$$
\begin{gathered}
E_{x}=E_{0}\left(C e^{-\gamma_{1} z}+D e^{\gamma_{1} z}\right), \\
H_{y}=\frac{1}{\eta_{1}} E_{0}\left(C e^{-\gamma_{1} z}-D e^{\gamma_{1} z}\right),
\end{gathered}
$$

where $C$ and $D$ are the coefficients dependent on the permittivity and permeability of materials. They can be determined by applying boundary conditions described by eqs. 
(4.17) and (4.18) in the general solution. The coefficients $C$ and $D$ are then solved and given as

$$
\begin{gathered}
C=\frac{T_{t}}{1-R_{r}^{2} e^{-2 \gamma_{1} L}}, \\
D=\frac{-T_{t} R_{r} e^{-2 \gamma_{1} L}}{1-R_{r}^{2} e^{-2 \gamma_{1} L}},
\end{gathered}
$$

where $T_{t}$ and $R_{r}$ are the transmission and reflection coefficients at the interface between free space and magnetic dielectric medium, respectively, with the following forms:

$$
\begin{gathered}
T_{t}=\frac{2 \eta_{1}}{\eta_{1}+\eta_{0}}, \\
R_{r}=\frac{\eta_{1}-\eta_{0}}{\eta_{1}+\eta_{0}} .
\end{gathered}
$$

The electric field within material can thus be obtained as

$$
E_{x}=E_{0} \frac{T_{t}\left(e^{-\gamma_{1} z}-R_{r} e^{-2 \gamma_{1} L+\gamma_{1} z}\right)}{1-R_{r}^{2} e^{-2 \gamma_{1} L}} .
$$

Both $T_{t}$ and $R_{r}$ are complex quantities and thus can be represented as

$$
\begin{gathered}
T_{t}=\left|T_{t}\right| e^{j \tau}, \\
R_{r}=\left|R_{r}\right| e^{j \delta},
\end{gathered}
$$

where $\tau$ and $\delta$ are the phase angles for transmission coefficient and reflection coefficient, respectively. With above definitions for $T_{t}, R_{r}$ and $\gamma$, eq. (4.29) is then transformed as 


$$
E_{x}=E_{0}\left|T_{t}\right| \frac{e^{-\alpha z} e^{j(\tau-\beta z)}-\left|R_{r}\right| e^{-\alpha(2 L-z)} e^{j[\tau+\delta-\beta(2 L-z)]}}{1-\left|R_{r}\right|^{2} e^{-2 \alpha L} e^{j(2 \delta-2 \beta L)}}
$$

In microwave heating, the contribution of dielectric loss $Q_{E}$ to the power dissipated (heat generated) per unit volume $\left(\mathrm{W} \mathrm{m}^{-3}\right)$ is determined by the Poynting vector. ${ }^{134}$ It can be simplified as

$$
Q_{E}(z)=\frac{1}{2} \omega \varepsilon_{0} \varepsilon_{r} "\left|E_{x}\right|^{2}
$$

Thus, $Q_{E}$ is determined by the following formula:

$$
\begin{aligned}
& Q_{E}(z)=\frac{1}{2} \omega \varepsilon_{0} \varepsilon_{r}{ }^{\prime}\left|E_{0}\right|^{2}\left|T_{t}\right|^{2} \times \\
& \frac{e^{-2 \alpha z}-2\left|R_{r}\right| e^{-2 \alpha L} \cos (2 \beta L-2 \beta z-\delta)+\left|R_{r}\right|^{2} e^{-2 \alpha L} e^{-2 \alpha(L-z)}}{1-2\left|R_{r}\right|^{2} e^{-2 \alpha L} \cos (2 \beta L-2 \delta)+\left|R_{r}\right|^{4} e^{-4 \alpha L}} .
\end{aligned}
$$

For microwave heating of magnetic dielectric materials the magnetic loss depends on the permeability. Using eqs. (4.24) and (4.29), the magnetic field within the materials can be solved and given by

$$
H_{y}=\frac{E_{0}}{\eta_{1}} \frac{T_{t}\left(e^{-\gamma_{1} z}+R_{r} e^{-2 \gamma_{1} L+\gamma_{1} z}\right)}{1-R_{r}^{2} e^{-2 \gamma_{1} L}} .
$$

Considering the phase angle of impedance of the magnetic dielectric medium $(\theta$, rad), eq. (4.35) can be transformed as

$$
H_{y}=\frac{E_{0}}{\left|\eta_{1}\right|}\left|T_{t}\right| \frac{e^{-\alpha z} e^{j(\tau-\beta z)}+\left|R_{r}\right| e^{-\alpha(2 L-z)} e^{j[\tau+\delta-\beta(2 L-z)]}}{e^{j \theta}-\left|R_{r}\right|^{2} e^{-2 \alpha L} e^{j(\theta+2 \delta-2 \beta L)}} .
$$


Similarly, the contribution of magnetic loss $Q_{H}$ to the power dissipated (heat generated) per unit volume $\left(\mathrm{W} \mathrm{m}^{-3}\right)$ can also be determined by the Poynting vector. It is simplified as

$$
Q_{H}(z)=\frac{1}{2} \omega \mu_{0} \mu_{r} "\left|H_{y}\right|^{2} .
$$

The magnetic loss in the microwave heating of the magnetic dielectric material can then be determined as

$$
\begin{aligned}
& Q_{H}(z)=\frac{1}{2} \omega \mu_{0} \mu_{r} " \frac{\left|E_{0}\right|^{2}}{\left|\eta_{1}\right|^{2}}\left|T_{t}\right|^{2} \times \\
& \frac{e^{-2 \alpha z}+2\left|R_{r}\right| e^{-2 \alpha L} \cos (2 \beta L-2 \beta z-\delta)+\left|R_{r}\right|^{2} e^{-2 \alpha L} e^{-2 \alpha(L-z)}}{1-2\left|R_{r}\right|^{2} e^{-2 \alpha L} \cos (2 \beta L-2 \delta)+\left|R_{r}\right|^{4} e^{-4 \alpha L}} .
\end{aligned}
$$

The total heat generation $Q_{z}$ throughout the magnetic dielectric materials under microwave irradiation can thus be determined by the following equation:

$$
\begin{gathered}
Q_{z}=Q_{E}(z)+Q_{H}(z), \\
Q_{z}=\frac{1}{2} \omega \varepsilon_{0} \varepsilon_{r} "\left|E_{0}\right|^{2}\left|T_{t}\right|^{2} \frac{e^{-2 \alpha z}-2\left|R_{r}\right| e^{-2 \alpha L} \cos (2 \beta L-2 \beta z-\delta)+\left|R_{r}\right|^{2} e^{-2 \alpha L} e^{-2 \alpha(L-z)}}{1-2\left|R_{r}\right|^{2} e^{-2 \alpha L} \cos (2 \beta L-2 \delta)+\left|R_{r}\right|^{4} e^{-4 \alpha L}} \\
+\left.\frac{1}{2} \omega \mu_{0} \mu_{r}{ }^{\prime}\left|\frac{\left|E_{0}\right|^{2}}{\left|\eta_{1}\right|^{2}}\right| T_{t}\right|^{2} \frac{e^{-2 \alpha z}+2\left|R_{r}\right| e^{-2 \alpha L} \cos (2 \beta L-2 \beta z-\delta)+\left|R_{r}\right|^{2} e^{-2 \alpha L} e^{-2 \alpha(L-z)}}{1-2\left|R_{r}\right|^{2} e^{-2 \alpha L} \cos (2 \beta L-2 \delta)+\left|R_{r}\right|^{4} e^{-4 \alpha L}} .
\end{gathered}
$$

To demonstrate the contribution of magnetic loss to microwave heating of magnetic dielectric materials, the dielectric and magnetic losses of five ferrites, namely, barium ferrite $\left(\mathrm{BaFe}_{12} \mathrm{O}_{19}\right)$, strontium ferrite $\left(\mathrm{SrFe}_{12} \mathrm{O}_{19}\right)$, copper ferrite $\left(\mathrm{CuFe}_{2} \mathrm{O}_{4}\right)$, copper zinc ferrite $\left(\mathrm{CuZnFe}_{4} \mathrm{O}_{4}\right)$ and nickel zinc ferrite $\left(\mathrm{NiZnFe}_{4} \mathrm{O}_{4}\right)$ were determined using the 
formulas derived above. Ferrites were chosen because: 1) they are important for preparation of various electronic and microwave devices and 2) synthesis of ferrites by microwave heating has been widely studied. ${ }^{135-137}$ The permittivity and permeability of these ferrites are summarized in Table $4.1 .^{138^{*}}$

Table 4.1

Permittivity and permeability of the ferrites at 2-40 GHz.

\begin{tabular}{lllll}
\hline Ferrite & $\varepsilon_{r}{ }^{\prime}$ & $\varepsilon_{r}{ }^{\prime \prime}$ & $\mu_{r}{ }^{\prime}$ & $\mu_{r}{ }^{\prime \prime}$ \\
\hline $\mathrm{BaFe}_{12} \mathrm{O}_{19}$ & 1.4647 & 0.0516 & 1.1046 & 0.0706 \\
$\mathrm{SrFe}_{12} \mathrm{O}_{19}$ & 1.4061 & 0.04465 & 1.0941 & 0.0730 \\
$\mathrm{CuFe}_{2} \mathrm{O}_{4}$ & 1.5073 & 0.0906 & 1.029 & 0.1061 \\
$\mathrm{CuZnFe}_{4} \mathrm{O}_{4}$ & 1.5951 & 0.0888 & 1.0376 & 0.2092 \\
$\mathrm{NiZnFe}_{4} \mathrm{O}_{4}$ & 1.3132 & 0.1232 & 1.0797 & 0.2012 \\
\hline
\end{tabular}

*Average values were used due to slight frequency dependences of permittivity and permeability.

Table 4.2 shows the microwave absorption parameters of the ferrites at $2450 \mathrm{MHz}$, which were determined based on the calculations using the formulas developed above with the values of corresponding permittivity and permeability. It is seen that all the ferrites have high microwave transmission coefficients, suggesting a partial contribution to efficient microwave heating. To confirm this effect, it is necessary to characterize the field distributions in the ferrites, as presented in Fig. 4.2. The incident microwave energy flux $\left(I=c \varepsilon_{0} E_{0}{ }^{2} / 2, c\right.$ is the speed of microwave in free space) was kept constant at $3 \mathrm{~W} \mathrm{~cm}^{-2}$, which corresponds to a $1.2 \mathrm{~kW}$ household microwave. ${ }^{134}$ This gives the incident electric field $\left(E_{0}\right)$ having an amplitude of $4754 \mathrm{~V} \mathrm{~m}^{-1}$. 
Table 4.2

Microwave absorption parameters of the ferrites at $2450 \mathrm{MHz}$.

\begin{tabular}{llllll}
\hline Properties & $\mathrm{BaFe}_{12} \mathrm{O}_{19}$ & $\mathrm{SrFe}_{12} \mathrm{O}_{19}$ & $\mathrm{CuFe}_{2} \mathrm{O}_{4}$ & $\mathrm{CuZnFe}_{4} \mathrm{O}_{4}$ & $\mathrm{NiZnFe}_{4} \mathrm{O}_{4}$ \\
\hline$\left|T_{t}\right|$ & 0.9300 & 0.9378 & 0.9058 & 0.8980 & 0.9545 \\
$\left|R_{r}\right|$ & 0.0704 & 0.0628 & 0.0949 & 0.1086 & 0.0511 \\
$\tau(\mathrm{rad})$ & -0.0077 & -0.0093 & -0.0117 & -0.0395 & -0.0237 \\
$\delta(\mathrm{rad})$ & -3.0404 & -3.0029 & -3.0298 & -2.8090 & -2.6824 \\
$\alpha(\mathrm{Np} / \mathrm{m})$ & 3.2351 & 3.1332 & 5.2140 & 8.4702 & 8.5501 \\
$\beta(\mathrm{rad} / \mathrm{m})$ & 65.2749 & 63.6543 & 63.9193 & 66.1863 & 61.1642 \\
$\left|\eta_{1}\right|(\Omega)$ & 327.3945 & 332.6047 & 311.8169 & 306.6524 & 343.7765 \\
\hline
\end{tabular}

As expected, Fig. 4.2 shows that the electric and magnetic fields keep relatively high values after transmission and reflection at the air-ferrite interface due to high transmission coefficients. It is also noticed that microwave fields of $\mathrm{CuZnFe}_{4} \mathrm{O}_{4}$ and $\mathrm{NiZnFe}_{4} \mathrm{O}_{4}$ dissipate faster than the others in the $0.05-\mathrm{m}$-thick slabs. This is mainly attributed to their shallower microwave penetration depths $\left(D_{p}=1 / 2 \alpha\right){ }^{65}$ The faster field dissipation would probably lead to high microwave heat power generation (high dielectric loss and/or magnetic loss) in the materials. 

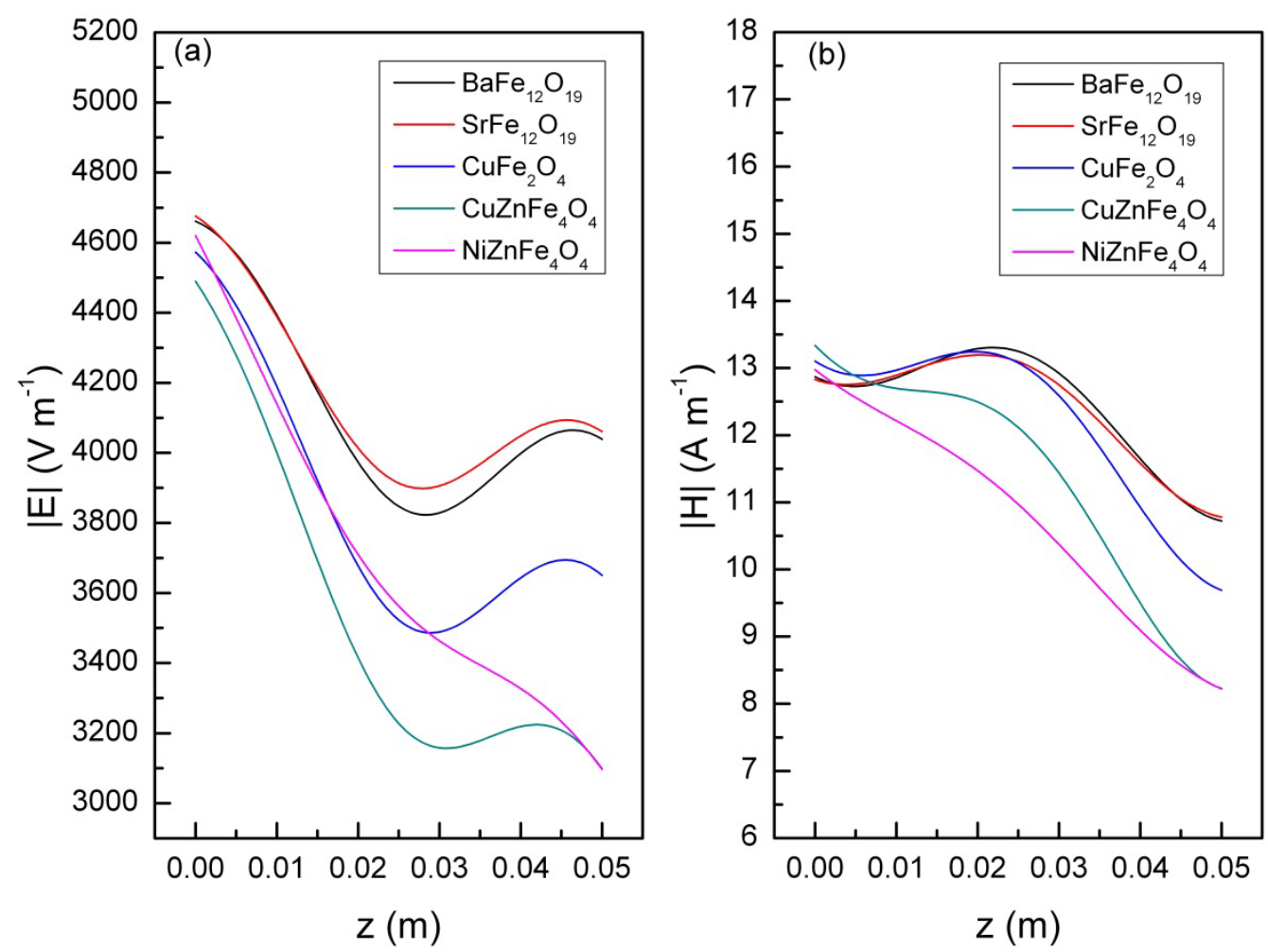

Fig. 4.2. (a) Electric field and (b) magnetic field distributions for microwave heating of the 0.05-m-thick ferrite slabs.

Figure 4.3 shows the dielectric loss and magnetic loss distributions for microwave heating of the 0.05 -m-thick ferrite slabs. Inspection of the figure indicates that magnetic losses for the ferrites are much larger than the corresponding dielectric losses $\left(\mathrm{Q}_{\mathrm{H}} \gg>\right.$ $\mathrm{Q}_{\mathrm{E}}$ ). The magnetic loss can be up to approximately four times greater than dielectric loss (e.g., $\left.\mathrm{CuZnFe}_{4} \mathrm{O}_{4}\right)$. It suggests that the magnetic loss is more important during the heating than the dielectric loss. This result is in good agreement with the observation in the experimental work where magnetic dielectric materials are heated much faster in the magnetic field than in the electric field of the microwave applicator. Hence, through our calculations based on the derived formula, it can be concluded that more efficient 
heating for magnetic dielectric materials using microwave magnetic field could be achieved. Also, note that $\mathrm{CuZnFe}_{4} \mathrm{O}_{4}$ and $\mathrm{NiZnFe}_{4} \mathrm{O}_{4}$ exhibit much larger magnetic losses than the others. It is thus expected that the ferrites have the following heating rate order in microwave magnetic field heating: $\mathrm{CuZnFe}_{4} \mathrm{O}_{4}>\mathrm{NiZnFe}_{4} \mathrm{O}_{4}>\mathrm{CuFe}_{2} \mathrm{O}_{4}>$ $\mathrm{SrFe}_{12} \mathrm{O}_{19} \approx \mathrm{BaFe}_{12} \mathrm{O}_{19}$.
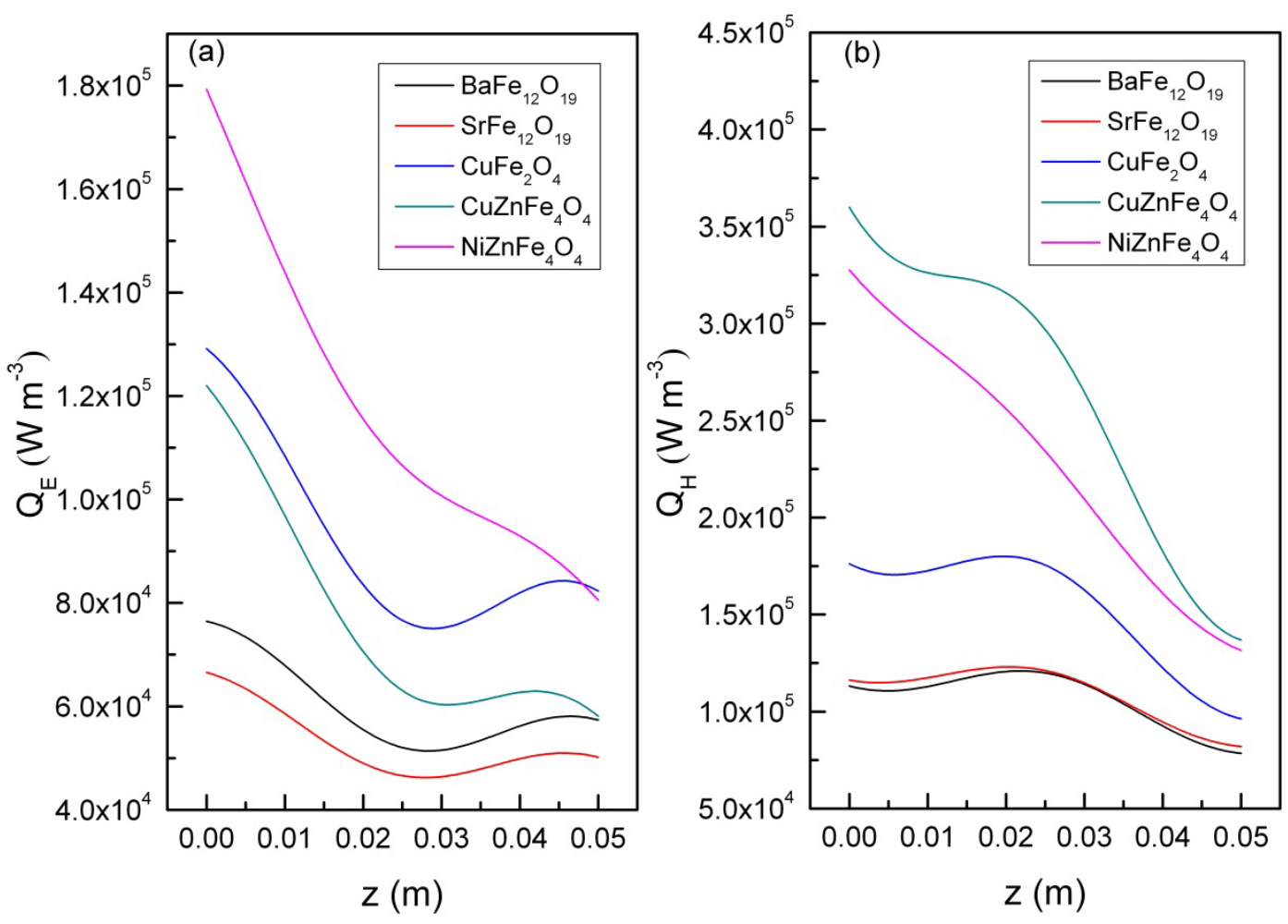

Fig. 4.3. (a) Dielectric loss and (b) magnetic loss distributions for microwave heating of the 0.05-m-thick ferrite slabs.

In summary, a simplified equation for determining magnetic loss in materials subjected to microwave irradiation was derived in this work. The magnetic losses for five ferrites including $\mathrm{BaFe}_{12} \mathrm{O}_{19}, \mathrm{SrFe}_{12} \mathrm{O}_{19}, \mathrm{CuFe}_{2} \mathrm{O}_{4}, \mathrm{CuZnFe}_{4} \mathrm{O}_{4}$ and $\mathrm{NiZnFe}_{4} \mathrm{O}_{4}$ were calculated 
at $2450 \mathrm{MHz}$ using the derived theoretical formula. The calculations of loss distributions in the ferrites show that magnetic loss is up to approximately four times greater than the dielectric loss in the microwave heating of ferrites. This demonstrates that magnetic loss exhibits a primary effect on the microwave heating of ferrites, indicating that efficient heating could be achieved in the microwave magnetic field. Additionally, due to the large difference of magnetic losses between the ferrites, it is anticipated that the ferrites have the following heating rate order in microwave magnetic field heating: $\mathrm{CuZnFe}_{4} \mathrm{O}_{4}>\mathrm{NiZnFe}_{4} \mathrm{O}_{4}>\mathrm{CuFe}_{2} \mathrm{O}_{4}>\mathrm{SrFe}_{12} \mathrm{O}_{19} \approx \mathrm{BaFe}_{12} \mathrm{O}_{19}$. The developed formula is not only beneficial to the analysis of the heating characteristics of materials in the microwave magnetic field but also useful in the future investigation of the heat transfer process in the microwave heating of magnetic dielectrics. 


\title{
Chapter 5 Microwave Absorption Properties of Materials for
}

\author{
Ironmaking
}

\begin{abstract}
The content of this chapter was previously published in ISIJ International ${ }^{139}$ by Zhiwei Peng, JiannYang Hwang, Chong-Lyuck Park, Byoung-Gon Kim, Matthew Andriese, Xinli Wang and in ISIJ International ${ }^{65}$ by Zhiwei Peng, Jiann-Yang Hwang, Joe Mouris, Ron Hutcheon, Xiaodi Huang and in Metallurgical and Materials Transactions $\mathrm{A}^{140}$ by Zhiwei Peng, Jiann-Yang Hwang, Joe Mouris, Ron Hutcheon, Xiang Sun and in TMS Annual Meeting, 3rd International Symposium on High-Temperature Metallurgical Processing - Held During the TMS 2012 Annual Meeting and Exhibition ${ }^{141}$ by Zhiwei Peng, Jiann-Yang Hwang, Zheng Zhang, Matthew Andriese, Xiaodi Huang and in Energy \& Fuels by Zhiwei Peng, Jiann-Yang Hwang, Byoung-Gon Kim, Joe Mouris, Ron Hutcheon. ${ }^{142}$
\end{abstract}

Reproduced with permission from ISIJ International: Zhiwei Peng, Jiann-Yang Hwang, Chong-Lyuck Park, Byoung-Gon Kim, Matthew Andriese, Xinli Wang. Microwave Permittivity, Permeability, and Absorption Capability of Ferric Oxide. ISIJ International. 2012;52(9):1541-1544. Copyright (C) 2012 The Iron and Steel Institute of Japan. (See Appendix C-1 for copyright permission).

Reproduced with permission from ISIJ International: Zhiwei Peng, Jiann-Yang Hwang, Joe Mouris, Ron Hutcheon, Xiaodi Huang. Microwave Penetration Depth in Materials with Non-zero Magnetic Susceptibility. ISIJ International. 2010;50(11):1590-1596. Copyright (C) 2010 The Iron and Steel Institute of Japan. (See Appendix C-2 for copyright permission).

Reproduced with permission from Metallurgical and Materials Transactions A: Zhiwei Peng, Jiann-Yang Hwang, Joe Mouris, Ron Hutcheon, Xiang Sun. Microwave Absorption Characteristics of Conventionally Heated Nonstoichiometric Ferrous Oxide. Metallurgical and Materials Transactions A. 2011;42A(8):2259-2263. Copyright (C) 2011 The Minerals, Metals and Materials Society. (See Appendix C-3 for copyright permission).

Reproduced with permission from TMS Annual Meeting, 3rd International Symposium on HighTemperature Metallurgical Processing - Held During the TMS 2012 Annual Meeting and Exhibition: Zhiwei Peng, Jiann-Yang Hwang, Zheng Zhang, Matthew Andriese, Xiaodi Huang. Thermal Decomposition and Regeneration of Wüstite. TMS Annual Meeting, 3rd International Symposium on High-Temperature Metallurgical Processing - Held During the TMS 2012 Annual Meeting and Exhibition. 2012;147-156. Copyright (C) 2012 The Minerals, Metals and Materials Society. (See Appendix C-4 for copyright permission).

Reproduced with permission from Energy \& Fuels: Zhiwei Peng, Jiann-Yang Hwang, Byoung-Gon Kim, Joe Mouris, Ron Hutcheon. Microwave Absorption Capability of High Volatile Bituminous Coal during Pyrolysis. Energy \& Fuels. 2012;26(8):5146-5151. Copyright (C) 2012 The American Chemical Society. (See Appendix C-5 for copyright permission). 


\subsection{Hematite}

\subsubsection{Permittivity and Permeability of Hematite}

Hematite (ferric oxide, $99.98 \%$ purity) powders were supplied by Sigma-Aldrich Corp., St. Louis, MO. The chemical composition was confirmed by X-ray diffraction (XRD) using a conventional Scintag XDS2000 powder X-ray diffractometer (Scintag Inc., Cupertino, CA) with a graphite monochromator and $\mathrm{Cu} K_{\alpha}$ radiation. The sample microstructure and particle distribution were characterized by using a Hitachi S-4700 field-emission scanning electron microscope (FE-SEM, Hitachi Ltd., Tokyo, Japan).

The permittivity and permeability of the $\mathrm{Fe}_{2} \mathrm{O}_{3}$ sample were measured by the cavity perturbation technique. ${ }^{65}$ As for the permittivity test, $\mathrm{Fe}_{2} \mathrm{O}_{3}$ powders were first uniaxially pressed at $\sim 207 \mathrm{MPa}$ in a die lined with tungsten carbide to form pellets with a diameter of $\sim 3.62 \mathrm{~mm}$ having a total stacked length (height) of $12.97 \mathrm{~mm}$. The bulk density (room temperature) of the sample was $2.79 \mathrm{~g} \mathrm{~cm}^{-3}$. During the measurements, the sample was step-heated in the conventional resistance furnace to the designated temperatures in $0.01 \mathrm{~L} \mathrm{~min}^{-1}$ flowing argon. The permittivity measurements started at room temperature $\left(24{ }^{\circ} \mathrm{C}\right.$ ) and heated in $50{ }^{\circ} \mathrm{C}$ steps to $\sim 1100{ }^{\circ} \mathrm{C}$. In the permeability measurements, the same punch/die unit was used to form pellets with a diameter of about $3.62 \mathrm{~mm}$ and total length of $13.38 \mathrm{~mm}$. The bulk density of pellets (room 
temperature) was $2.80 \mathrm{~g} \mathrm{~cm}^{-3}$. The measurements were performed in the same argon, starting at room temperature, then in $50^{\circ} \mathrm{C}$ steps to $\sim 1000{ }^{\circ} \mathrm{C}$.

It is widely known that microwaves are electromagnetic radiation having a broad frequency range of 0.3 to $300 \mathrm{GHz}$. However, to avoid interference with communication networks, all microwave heaters (domestic or scientific) are designed to work at either 915 or $2450 \mathrm{MHz}$. Due to this reason, this study focuses on the characterizations of the permittivity and permeability at these two frequencies.

Figure 5.1 shows the XRD pattern of hematite (ferric oxide, $\mathrm{Fe}_{2} \mathrm{O}_{3}$ ). It is found that all of the marked peaks belong to the $\alpha$ phase, indicating the sample having rhombohedral structure. The morphology of $\mathrm{Fe}_{2} \mathrm{O}_{3}$ was characterized using FE-SEM, as presented in Fig. 5.2. It shows that the sample powders are spherical in shape and have particle sizes between 0.05 and $0.2 \mu \mathrm{m}$.

Figure 5.3 illustrates the temperature dependences of real part $\left(\varepsilon_{r}{ }^{\prime}\right)$ and imaginary part $\left(\varepsilon_{r}{ }^{\prime \prime}\right)$ of complex relative permittivity of $\mathrm{Fe}_{2} \mathrm{O}_{3}$ at 915 and $2450 \mathrm{MHz}$. It is seen that $\varepsilon_{r}{ }^{\prime}$ and $\varepsilon_{r}{ }^{\prime \prime}$ slightly increase with temperature up to $450{ }^{\circ} \mathrm{C}$. The examination and calibration of experimental data demonstrate that the dried $\mathrm{Fe}_{2} \mathrm{O}_{3}$ has the $\varepsilon_{r}{ }^{\prime \prime}$ values of about 0.014 and 0.012 at $24{ }^{\circ} \mathrm{C}$ for 915 and $2450 \mathrm{MHz}$, respectively. It suggests that $\mathrm{Fe}_{2} \mathrm{O}_{3}$ possesses a very low dielectric loss at room temperature. 


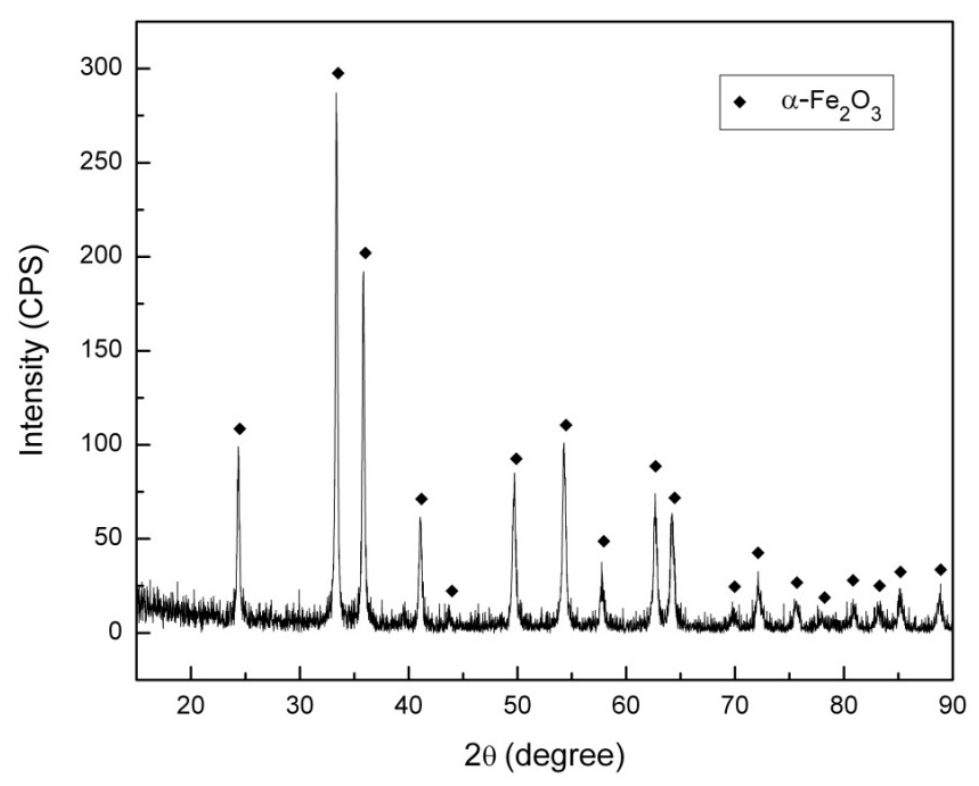

Fig. 5.1. XRD pattern of $\mathrm{Fe}_{2} \mathrm{O}_{3}$.

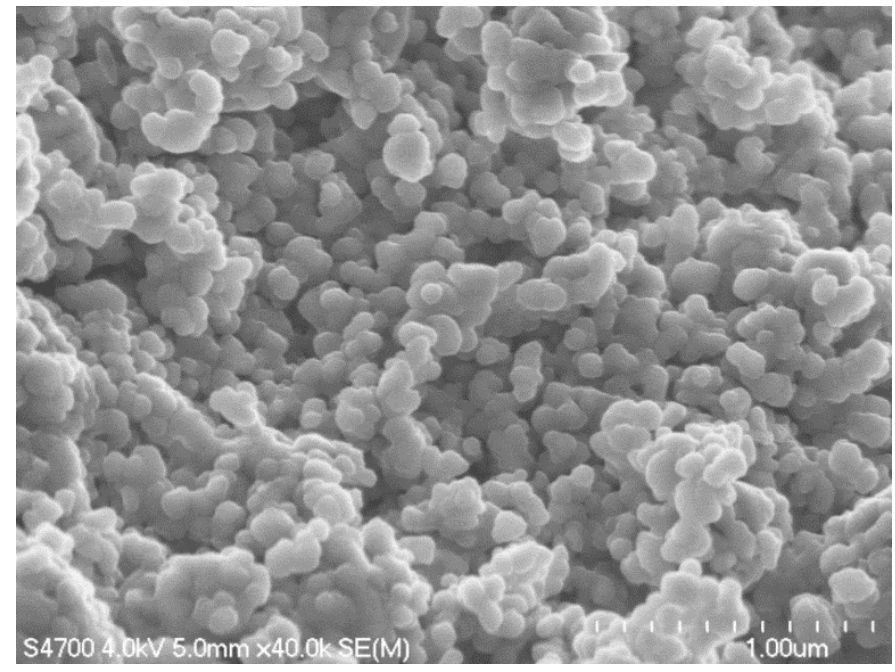

Fig. 5.2. Field emission-scanning electron microscope (FE-SEM) image of $\mathrm{Fe}_{2} \mathrm{O}_{3}$ particles. 


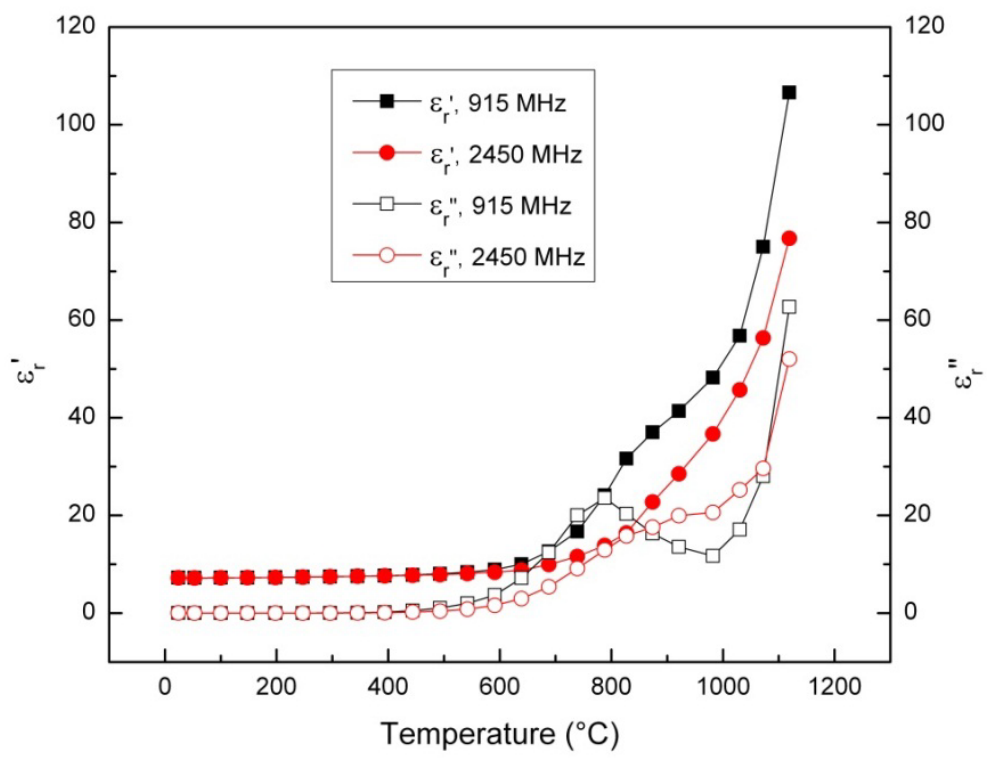

Fig. 5.3. Temperature dependence of complex relative permeability $\left(\varepsilon_{r}{ }^{\prime}\right.$ and $\left.\varepsilon_{r}{ }^{\prime \prime}\right)$ of $\mathrm{Fe}_{2} \mathrm{O}_{3}$.

Inspection of Fig. 5.3 shows that $\varepsilon^{\prime \prime}$ and $\varepsilon_{r}{ }^{\prime \prime}$ vary similarly below $450{ }^{\circ} \mathrm{C}$. As temperature increases, however, they begin to show different variation behaviors. The $\varepsilon_{r}{ }^{\prime}$ value rapidly increases with temperature beyond $450{ }^{\circ} \mathrm{C}$ while the $\varepsilon_{r}{ }^{\prime \prime}$ values show a broad dielectric loss peak between 450 and $1000{ }^{\circ} \mathrm{C}$. This is known as a typical relaxation/interfacial polarization phenomena behavior, usually indicating a change in the material associated with the loss of an insulating barrier between particles, or the presence of a transient species during a phase change. ${ }^{139,140}$ Such observation is associated with microstructure change due to sintering occurred at high temperatures, which can be demonstrated by the sample bulk density variation during the measurement, as depicted in Fig. 5.4. 


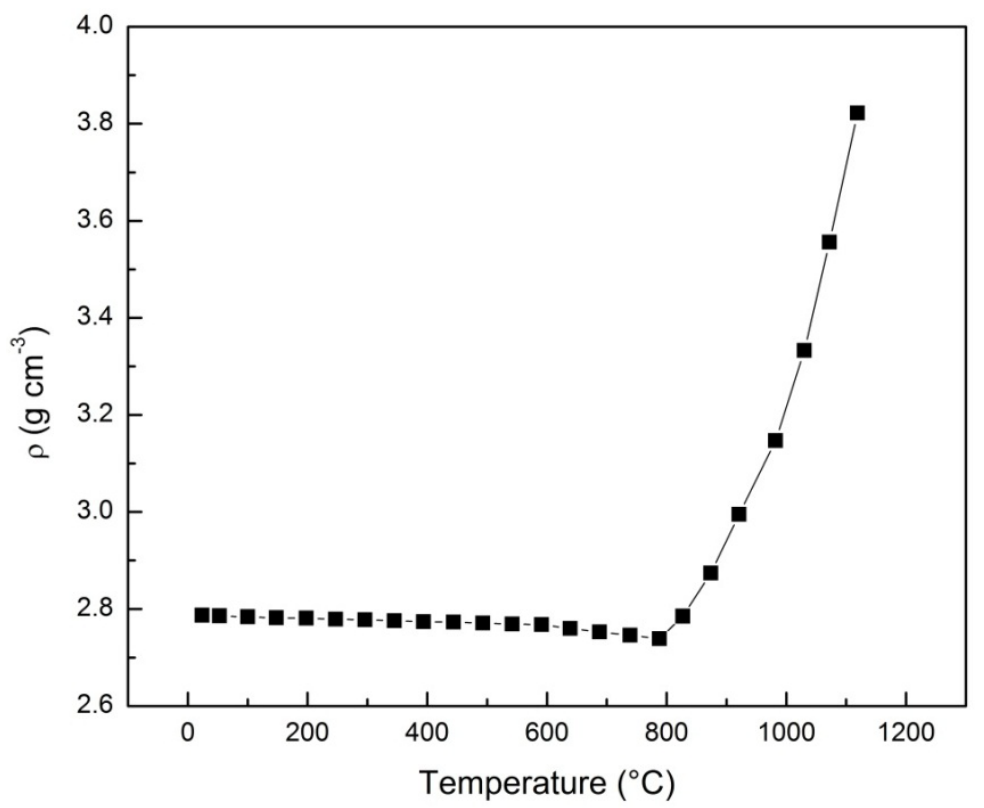

Fig. 5.4. Variation of bulk density of the sample pellet with temperature during the measurement.

Figure 5.4 shows that the sample bulk density $(\rho)$ remains constant until $800{ }^{\circ} \mathrm{C}$ and then increases proportionally with temperature. This suggests that sintering starts at 800 ${ }^{\circ} \mathrm{C}$ and becomes more evident at higher temperatures. Sintering makes the sample particles adhere together, resulting in a strong surface densification with the possibility of grain growth during extended heating time. The increase in the average grain size, as shown in Fig. 5.5, may hinder the diffusion of iron and oxygen ions, decreasing dielectric polarization. ${ }^{140}$ This effect is found to be more obvious above $1000{ }^{\circ} \mathrm{C}$. However, it should be emphasized that the measured dielectric loss of materials comprises of dipole/ion contribution $\left(\varepsilon_{r, d}^{\prime \prime}\right)$ and conductivity $(\sigma)$ contribution, as given by the following equation: ${ }^{143}$ 


$$
\varepsilon_{r}^{\prime \prime}=\varepsilon_{r, d}^{\prime \prime}+\frac{\sigma}{2 \pi f \varepsilon_{0}}
$$

Thus, one can expect that a possible increase in electrical conductivity due to thermal activation at high temperatures may overwhelm the adverse effect of sintering, leading to a high dielectric loss. This possibility can be confirmed by the fact that electrical conductivity of $\mathrm{Fe}_{2} \mathrm{O}_{3}$ increases with temperature, as expressed by the following formula: ${ }^{144}$

$$
\sigma=2.7 \times 10^{5} e^{\left(-0.96 / k_{B} T\right)}
$$

where $\sigma$ is the electrical conductivity, $k_{B}$ is the Boltzmann constant, and $T$ is the temperature (Kelvin).

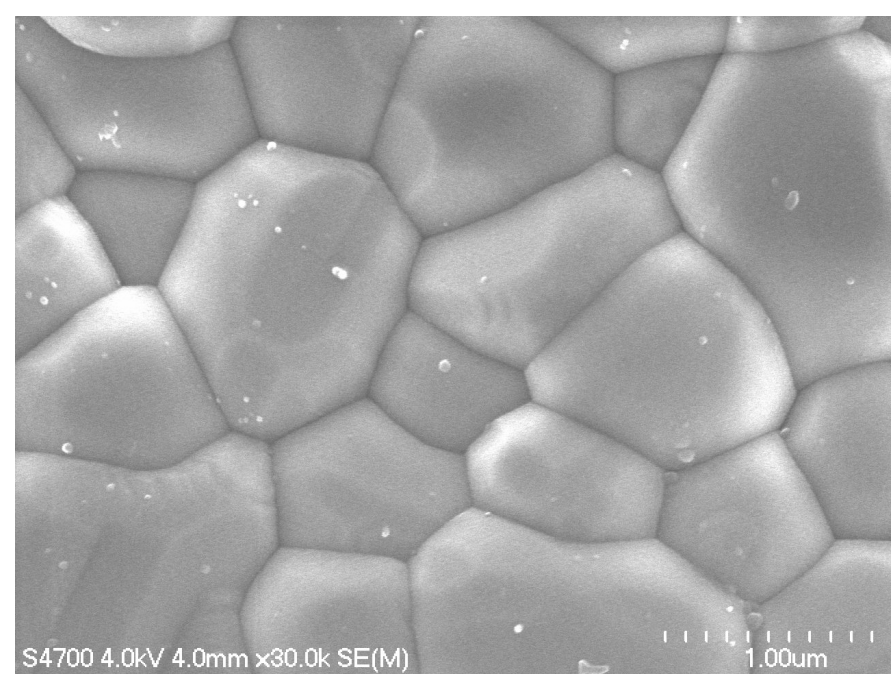

Fig. 5.5. FE-SEM image of the sintered sample pellet.

Figure 5.6 presents the permeability change in the temperature range between 24 and $1000{ }^{\circ} \mathrm{C}$. It reveals that both the real part $\left(\mu_{r}{ }^{\prime}\right)$ and imaginary part $\left(\mu_{r}{ }^{\prime \prime}\right)$ of complex 
relative permeability keep relatively constant below $700{ }^{\circ} \mathrm{C}$. Their values vary around 1 and 0 , respectively. It is also observed that there are small variations of permeability ( \pm 0.05 ) in the temperature range, resulting from the statistical error in the measurements. These results are consistent with the fact that $\mathrm{Fe}_{2} \mathrm{O}_{3}$ becomes weakly ferromagnetic above the Morin transition at $-13{ }^{\circ} \mathrm{C}$ and below its Néel temperature at $675{ }^{\circ} \mathrm{C} .145,146$ Continuous heating to higher temperatures gives rise to different variation behaviors of $\mu_{r}{ }^{\prime}$ and $\mu_{r}{ }^{\prime \prime}$. The $\mu_{r}{ }^{\prime}$ value decreases with increasing temperature beyond $700{ }^{\circ} \mathrm{C}$, which can be attributed to the increased electrical conductivity. As discussed before, the conductivity of $\mathrm{Fe}_{2} \mathrm{O}_{3}$ increases with temperature, making the sample more "conductive". According to Lenz's law, a time-varying magnetic field (e.g., microwave) induces large currents near the surface of a good conductor, producing a magnetic field opposite to the external magnetic field, leading to zero magnetic field inside. This means that a substance has an "effective magnetic susceptibility" of -1 , and thus appears to have a permeability value of $\mu_{r}{ }^{\prime} \approx 0$ when it exhibits high conductivity. Therefore, in the present case, the $\mu_{r}{ }^{\prime}$ values of $\mathrm{Fe}_{2} \mathrm{O}_{3}$ show a decreasing tendency as temperature increases. Contrary to $\mu_{r}^{\prime}$, it is noticed that the $\mu_{r}{ }^{\prime \prime}$ values stay negligible at temperatures higher than $700{ }^{\circ} \mathrm{C}$. This is because $\mathrm{Fe}_{2} \mathrm{O}_{3}$ exhibits paramagnetism above its Néel temperature. ${ }^{145}$ 
From above results, it can be inferred that $\mathrm{Fe}_{2} \mathrm{O}_{3}$ does not show noticeable magnetic response at 915 and $2450 \mathrm{MHz}$ below $1000{ }^{\circ} \mathrm{C}$. The dielectric loss is the primary factor contributing to the microwave absorption of $\mathrm{Fe}_{2} \mathrm{O}_{3}$ and the contribution of magnetic loss to microwave heating of $\mathrm{Fe}_{2} \mathrm{O}_{3}$ can be ignored.

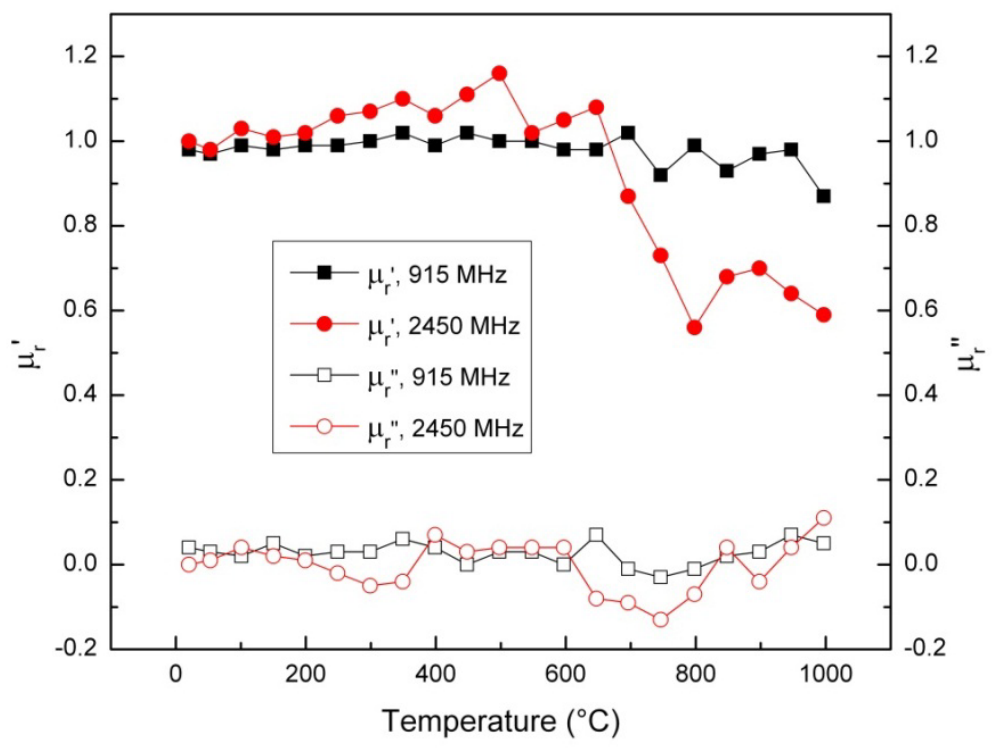

Fig. 5.6. Temperature dependence of complex relative permeability $\left(\mu_{r}{ }^{\prime}\right.$ and $\left.\mu_{r}{ }^{\prime \prime}\right)$ of $\mathrm{Fe}_{2} \mathrm{O}_{3}$.

\subsubsection{Microwave Absorption Capability of Hematite}

As aforementioned, microwave energy loss in materials relies on the permittivity and permeability. This suggests that a reasonable evaluation of microwave absorption capability of $\mathrm{Fe}_{2} \mathrm{O}_{3}$ has to take the combined effect of dielectric and magnetic properties. According to the previous studies, a quick evaluation of microwave 
absorption properties of materials can be achieved by determining the microwave penetration depth $\left(D_{p}\right){ }^{65}$ The equation for $D_{p}$ calculation is given by eq. (3.22).

Considering the negligible microwave magnetic loss of $\mathrm{Fe}_{2} \mathrm{O}_{3}\left(\mu_{\mathrm{r}}{ }^{\prime} \approx 1\right.$ and $\left.\mu_{\mathrm{r}}{ }^{\prime \prime} \approx 0\right)$ in the tested temperature range, the calculation of $D_{p}$ is determined by the variation of permittivity. Figure 5.7 shows the variation of $D_{p}$ with temperature. It is seen that $\mathrm{Fe}_{2} \mathrm{O}_{3}$ has large microwave penetration depths $(10.05$ and $4.36 \mathrm{~m}$ at 915 and $2450 \mathrm{MHz}$, respectively) at room temperature, indicating a very slow microwave dissipation inside the oxide. This is due to the small permittivity and permeability of $\mathrm{Fe}_{2} \mathrm{O}_{3}$ at low temperatures. As temperature increases, the $D_{p}$ value decreases rapidly mainly because of a significant increase in permittivity. Since the relative permittivity is much larger than the relative permeability at high temperatures, the permittivity dominates the variation of microwave penetration depth. Figure 5.6 indicates $D_{p}$ having values less than $0.045 \mathrm{~m}$ at both frequencies beyond $600^{\circ} \mathrm{C}$. This shows that ferric oxide undergoes a transition from a microwave transparent material to a good microwave absorber with increasing temperature. 


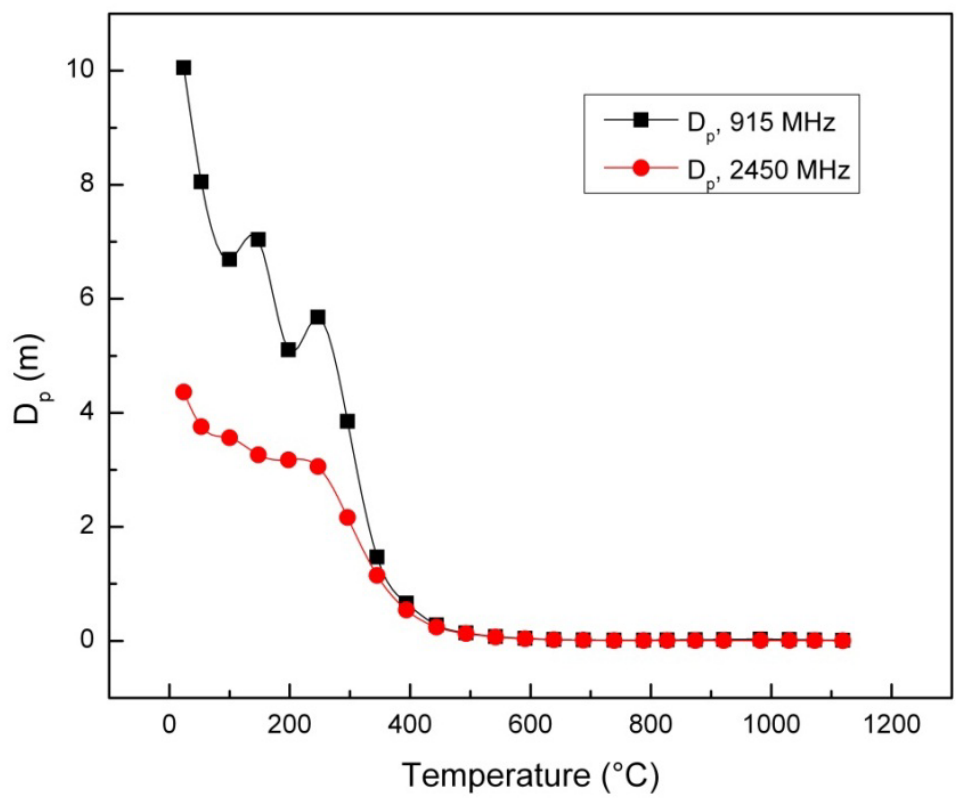

Fig. 5.7. Calculated microwave penetration depth of $\mathrm{Fe}_{2} \mathrm{O}_{3}$ as a function of temperature.

\subsubsection{Microwave Loss of Hematite}

As mentioned in Chapter 4, both dielectric and magnetic losses contribute to microwave heating. Because hematite is one of main materials for ironmaking, we also examine its dielectric loss and magnetic loss in a broad temperature range. To demonstrate the temperature effect, the calculations of microwave losses at various temperatures $(2450$ MHz, $1.2 \mathrm{~kW}$ ) were performed. The results are shown in Figs. 5.8 and 5.9. 


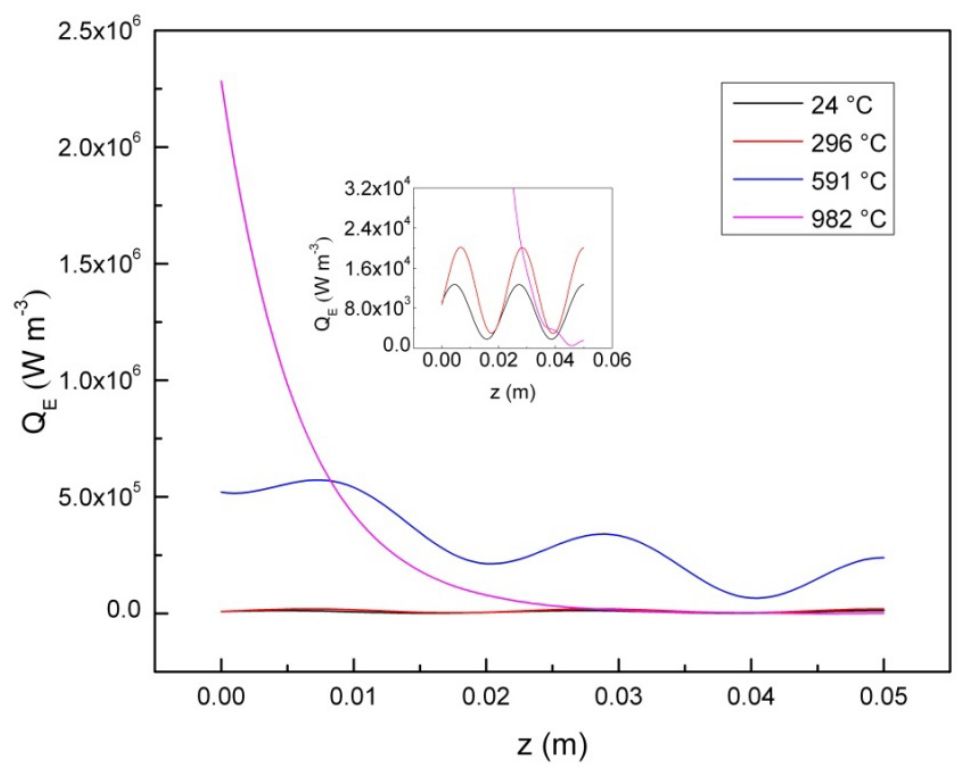

Fig. 5.8. Dielectric loss distributions for microwave heating of the 0.05-m-thick hematite slabs.

A comparison between dielectric loss and magnetic loss indicates that the dielectric loss of hematite is much larger than the magnetic loss of hematite. This trend becomes more apparent as temperature increases. Therefore, it can be concluded that microwave heating of hematite is mainly attributed to the dielectric loss of hematite. 


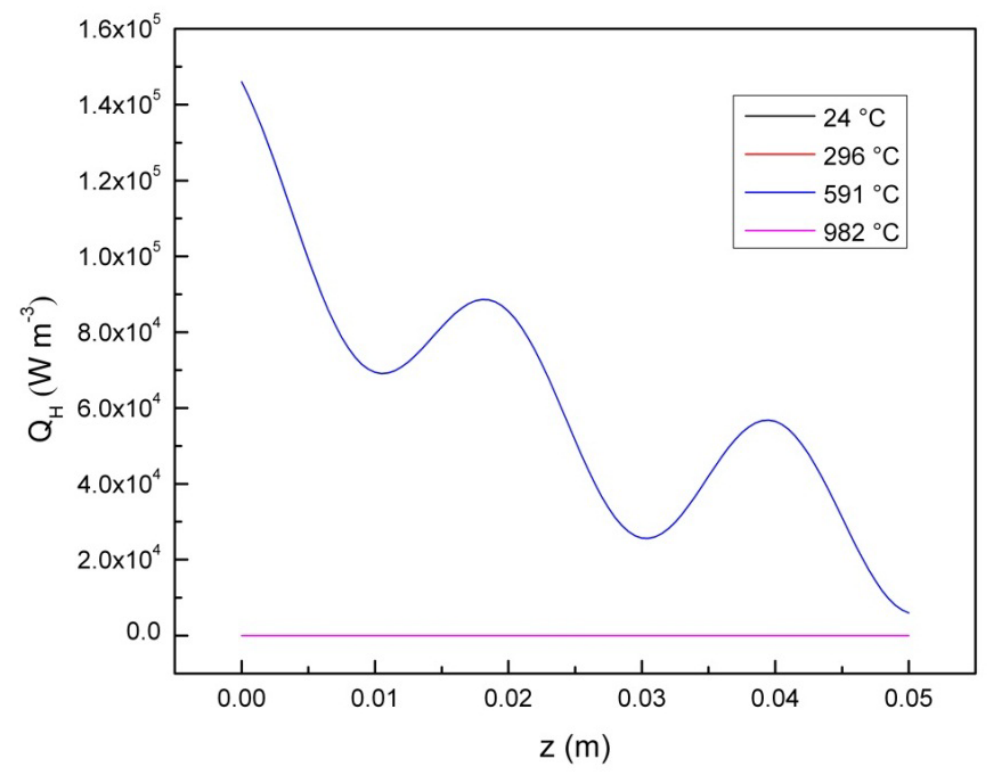

Fig. 5.9. Magnetic loss distributions for microwave heating of the 0.05 -m-thick hematite slabs.

\subsection{Magnetite Concentrate}

\subsubsection{Permittivity and Permeability of Magnetite Concentrate}

A typical ferromagnetic material, magnetite concentrate, obtained from the Tilden Mine in Michigan, was used to demonstrate the temperature and frequency dependences of dielectric and magnetic properties (permittivity and permeability, respectively). The phase compositions were determined using a Scintag XDS2000 powder x-ray diffractometer (Scintag Inc., Cupertino, CA) with a graphite monochromator and $\mathrm{Cu} K_{\alpha}$ radiation and the X-ray diffraction pattern is shown in Fig. 5.10. The analysis shows that the sample contains 3 phases, mainly magnetite $\left(\mathrm{Fe}_{3} \mathrm{O}_{4}, \mathrm{JCPDS}\right.$ card: $\left.79-0419\right)$ with 
a small amount of quartz ( $\mathrm{SiO}_{2}$, JCPDS card: $\left.88-2302\right)$ and siderite $\left(\mathrm{FeCO}_{3}, \mathrm{JCPDS}\right.$ card: 29-0696).

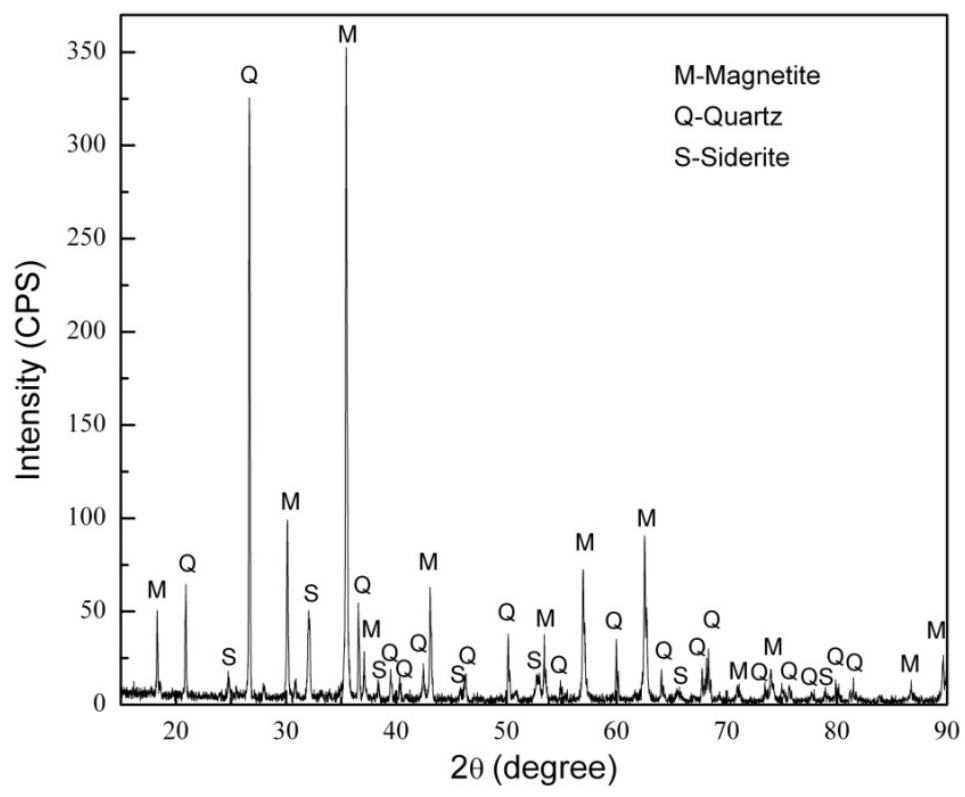

Fig. 5.10. X-ray diffraction pattern of magnetite concentrate.

In the permittivity measurement, magnetite concentrate powders with particle sizes less than $0.075 \mathrm{~mm}$ were uniaxially pressed at about $172 \mathrm{MPa}$ in a die lined with tungsten carbide to form 3 pellets with a diameter of about $3.63 \mathrm{~mm}$ and a total, stacked length (height) of $13.75 \mathrm{~mm}$. The bulk density (room temperature) of the pellets was $2.77 \mathrm{~g}$ $\mathrm{cm}^{-3}$. During the measurements, the pellets were step-heated in a resistance furnace to the designated temperatures in $0.01 \mathrm{~L} \mathrm{~min}^{-1}$ flowing argon. The present permittivity measurements were done from room temperature $\left(24{ }^{\circ} \mathrm{C}\right)$ in $\sim 50{ }^{\circ} \mathrm{C}$ steps to $1030{ }^{\circ} \mathrm{C}$, and then cooled down. 
The XRD after the measurement confirmed that no main phase changed during the heating. In the permeability experiment, the same punch/die unit was used to form pellets with a diameter of about $3.65 \mathrm{~mm}$ and length of $14.6 \mathrm{~mm}$. The bulk density of pellets (room temperature) was $2.84 \mathrm{~g} \mathrm{~cm}^{-3}$. The permeability measurements started at room temperature $\left(24^{\circ} \mathrm{C}\right.$ ) and used $\sim 50{ }^{\circ} \mathrm{C}$ steps to $850^{\circ} \mathrm{C}$. The argon gas flow was the same as that for permittivity test.

The measured values of the real and imaginary parts of complex relative permittivity and permeability of magnetite concentrate are shown in Figs. 5.11 and 5.12, respectively. In order to characterize the temperature dependences of the permittivity and permeability of magnetite, the curves in Figs. 5.11 and 5.12 were fitted to a polynomial equation of high degree (6), given as

$$
f(T)=a+b T+c T^{2}+d T^{3}+e T^{4}+f T^{5}+g T^{6},
$$

where $f(T)$ represents the temperature $\left(T,{ }^{\circ} \mathrm{C}\right)$ dependent permittivity $\left(\varepsilon_{r}{ }^{\prime}\right.$ and $\left.\varepsilon_{r}{ }^{\prime \prime}\right)$ or permeability $\left(\mu_{r}^{\prime}\right.$ and $\left.\mu_{r}{ }^{\prime \prime}\right)$, and the coefficients, $a, b, c, d, e, f$, and $g$, are constants. The variations of permittivity and permeability with temperature were characterized through the determination of the function. The fitted parameters are listed in Table 5.1. 


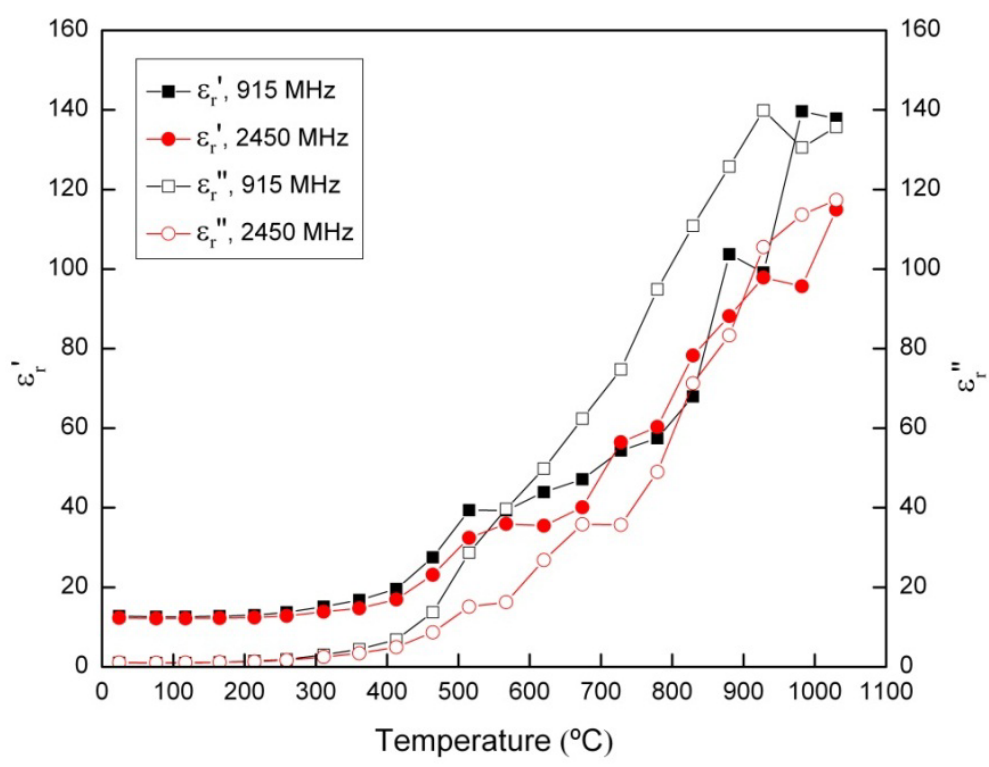

Fig. 5.11. Temperature dependence of complex relative permittivity of magnetite concentrate.

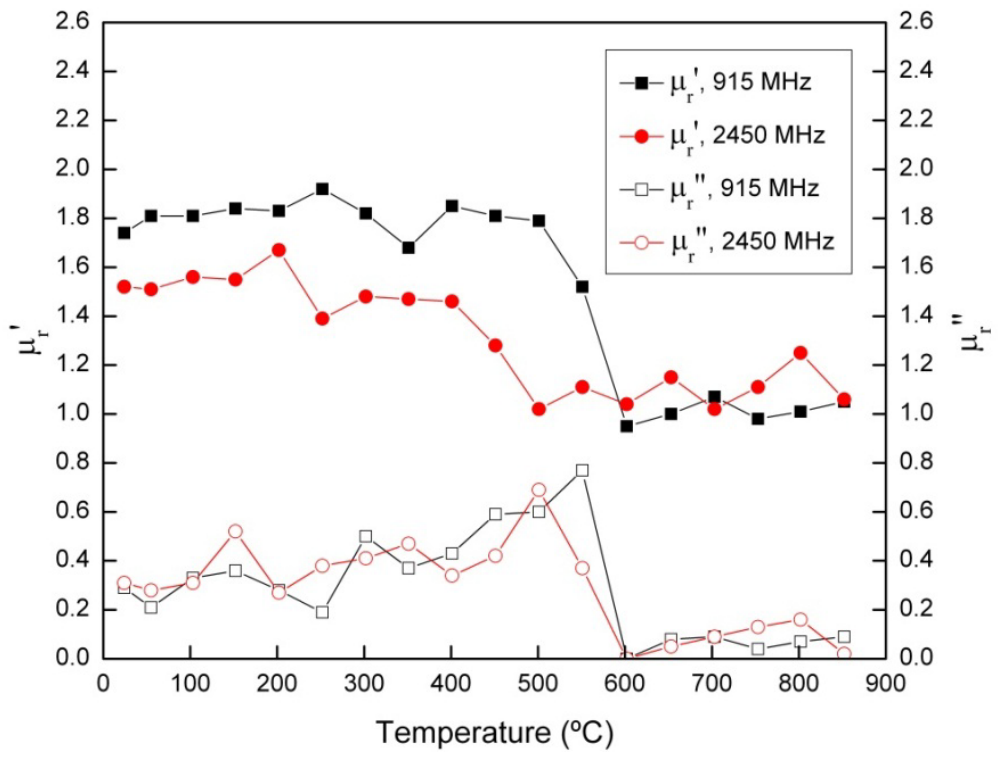

Fig. 5.12. Temperature dependence of complex relative permeability of magnetite concentrate. 
Table 5.1

Values of constants in the polynomial function (for $\varepsilon_{r}$ and $\mu_{r}$ ) represented by eq. (5.3).

\begin{tabular}{ccccccccc}
\hline$f(T)$ & $a$ & $b$ & $c / \times 10^{-4}$ & $d / \times 10^{-7}$ & $e / \times 10^{-9}$ & $f / \times 10^{-12}$ & $g / \times 10^{-16}$ & $R^{2}$ \\
\hline$\varepsilon_{\mathrm{r}}{ }^{\prime}(915 \mathrm{MHz})$ & 3.762 & 0.382 & -40.60 & 169.204 & -31.68 & 27.479 & -89.10 & 0.978 \\
$\varepsilon_{\mathrm{r}}{ }^{\prime}(2450 \mathrm{MHz})$ & 12.482 & 0.005 & -1.838 & 10.562 & -2.016 & 1.998 & -7.651 & 0.985 \\
$\varepsilon_{\mathrm{r}}{ }^{\prime}(915 \mathrm{MHz})$ & -1.086 & 0.085 & -8.021 & 27.600 & -3.966 & 3.343 & -12.82 & 0.995 \\
$\varepsilon_{\mathrm{r}}{ }^{\prime \prime}(2450 \mathrm{MHz})$ & -3.307 & 0.187 & -19.90 & 84.768 & -16.55 & 15.358 & -53.56 & 0.994 \\
$\mu_{\mathrm{r}}{ }^{\prime}(915 \mathrm{MHz})$ & 1.504 & 0.0113 & -1.229 & 5.758 & -1.273 & 1.293 & -4.887 & 0.890 \\
$\mu_{\mathrm{r}}{ }^{\prime}(2450 \mathrm{MHz})$ & 1.461 & 0.00234 & -0.225 & 1.139 & -0.312 & 0.390 & -1.751 & 0.828 \\
$\mu_{\mathrm{r}}{ }^{\prime \prime}(915 \mathrm{MHz})$ & 0.0094 & 0.0114 & -1.289 & 5.920 & -1.238 & 1.185 & -4.230 & 0.467 \\
$\mu_{\mathrm{r}}{ }^{\prime \prime}(2450 \mathrm{MHz})$ & 0.0478 & 0.0109 & -1.191 & 5.559 & -1.215 & 1.231 & -4.681 & 0.473 \\
\hline
\end{tabular}

The experimental data of relative permittivity and permeability were used to calculate the power penetration depths as a function of temperature at 915 and $2450 \mathrm{MHz}$ (Fig. 5.13). Below $600{ }^{\circ} \mathrm{C}$, the penetration depths determined by eq. (3.22) are much smaller than those calculated by eq. (1.5) as expected when the magnetic losses are included. Above $600{ }^{\circ} \mathrm{C}$, the difference between penetration depths determined by the two equations becomes negligible, reflecting the fact that the magnetic absorption is very small (in principle, zero) above the Curie point of magnetite $\left(585^{\circ} \mathrm{C}\right) .{ }^{147}$ For example, at $780{ }^{\circ} \mathrm{C}$ and $915 \mathrm{MHz}$, the penetration depths determined by eqs. (1.5) and (3.22) are 0.00505 and $0.00491 \mathrm{~m}$, respectively. This shows that the permeability should be considered in the determination of penetration depth of magnetic dielectrics, especially at temperatures below the dielectric's Curie point. 
It is useful to characterize the penetration depth with increasing temperature by curvefitting the values calculated by eq. (3.22). The temperature $\left(T,{ }^{\circ} \mathrm{C}\right)$ dependent penetration depth $\left(D_{p}\right.$, meters) can be expressed in the same function represented by eq. (5.3). Table 5.2 lists the values of constants yielding the polynomial function of penetration depth in meters.

Table 5.2

Values of constants in the polynomial function (for $D_{p}$ ) represented by eq. (5.3).

\begin{tabular}{ccccccccc}
\hline$D_{p}$ & $a$ & $b / \times 10^{-5}$ & $c / \times 10^{-7}$ & $d / \times 10^{-9}$ & $e / \times 10^{-11}$ & $f / \times 10^{-14}$ & $g / \times 10^{-18}$ & $R^{2}$ \\
\hline $915 \mathrm{MHz}$ & 0.0471 & -0.453 & 4.184 & -4.845 & 1.298 & -1.366 & 5.099 & 0.921 \\
& & & & & & & & \\
$2450 \mathrm{MHz}$ & 0.0161 & -1.863 & 2.182 & -1.603 & 0.395 & -0.405 & 1.502 & 0.901 \\
\hline
\end{tabular}

\subsubsection{Microwave Absorption Capability of Magnetite Concentrate}

As can be seen from Fig. 5.13, the optimum dimension for uniform heat deposition in a sample being irradiated from both sides in a $2450 \mathrm{MHz}$ microwave field is about $0.03 \mathrm{~m}$ (approximately two power penetration depths). The deposited microwave energy can be relatively uniformly distributed by double-sided irradiation in this dimension, and rapid relatively uniform temperature increases can be achieved. This is important since, with a larger sample, there will be obvious temperature gradients. In a smaller sample, a central hot spot may be produced by surface cooling. The accurate determination of penetration depth helps to optimize the load size in the microwave applicator. 


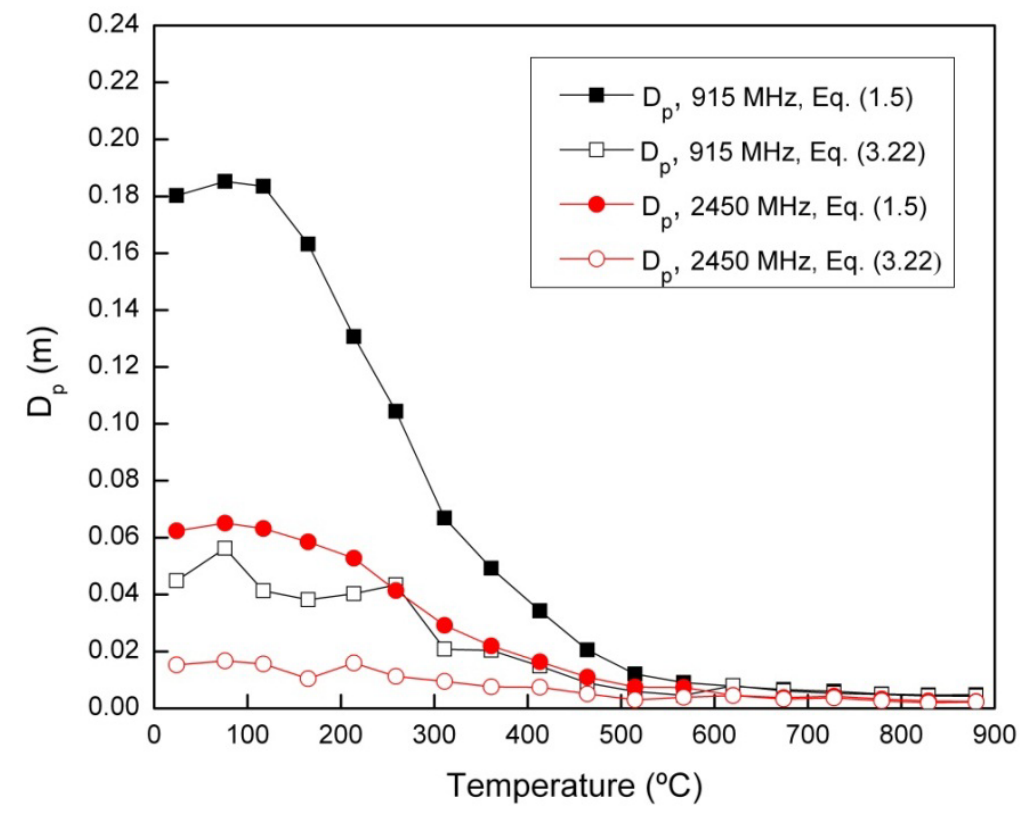

Fig. 5.13. Temperature dependence of microwave penetration depth of magnetite concentrate.

\subsubsection{Microwave Loss of Magnetite Concentrate}

Due to the strong magnetism of magnetite, it is expected that the magnetic loss of magnetite concentrate plays an important role in microwave heating. For this reason, a comparison between dielectric loss and magnetic loss of magnetite concentrate $(2450$ $\mathrm{MHz}, 1.2 \mathrm{~kW}$ ) were performed. The results are shown in Figs. 5.14 and 5.15. It is obvious that magnetic loss contributes more than dielectric loss to microwave heating of magnetite concentrate at low temperatures (e.g., $\left.24^{\circ} \mathrm{C}\right)$. However, as temperature goes up to the Curie point of magnetite, magnetic loss decreases significantly. Temperature increase in the sample is mainly ascribed to microwave dielectric loss beyond $600{ }^{\circ} \mathrm{C}$. 


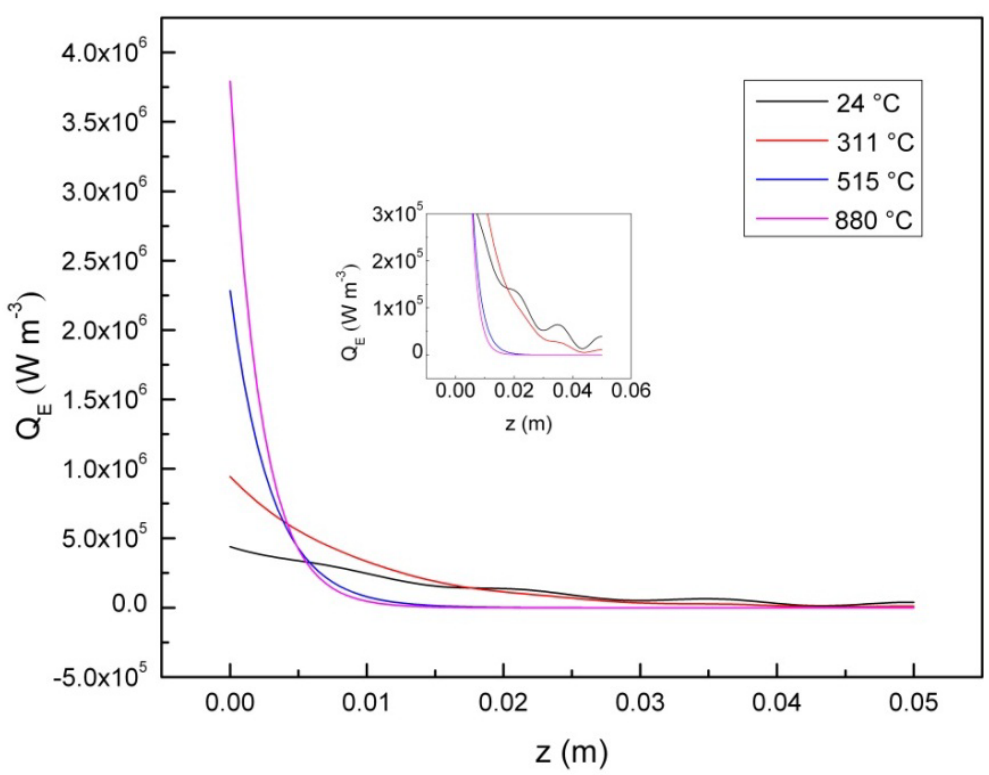

Fig. 5.14. Dielectric loss distributions for microwave heating of the 0.05-m-thick magnetite concentrate slabs.

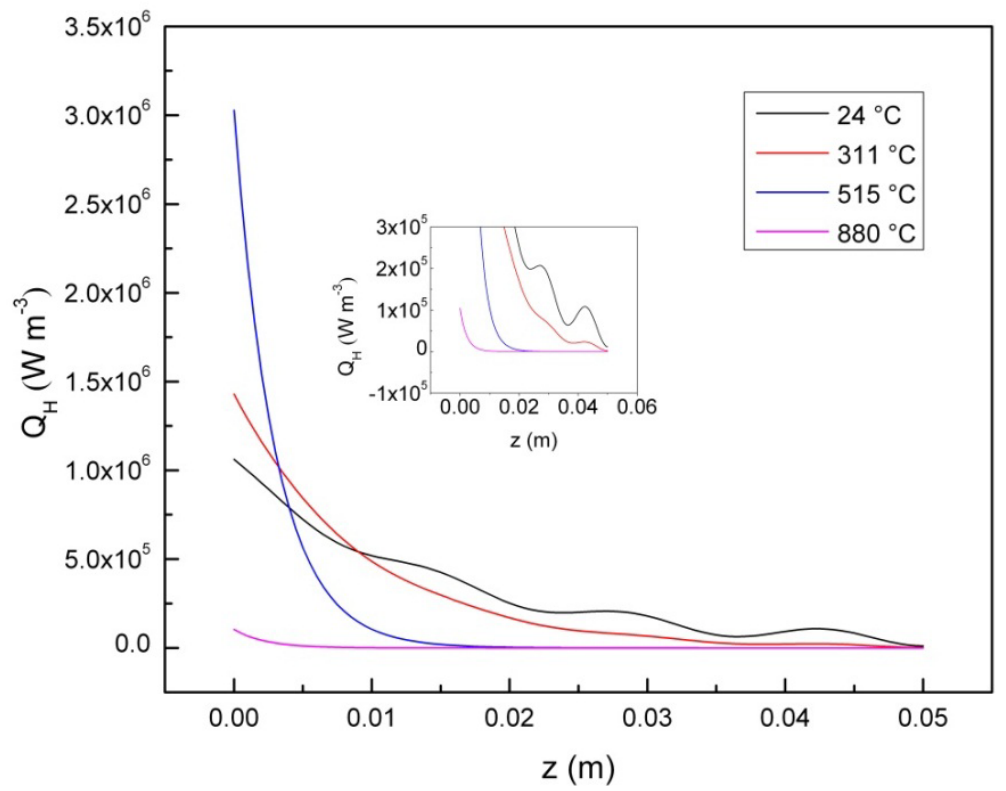

Fig. 5.15. Magnetic loss distributions for microwave heating of the 0.05-m-thick magnetite concentrate slabs. 


\subsection{Wüstite}

\subsubsection{Permittivity and Permeability of Wüstite}

Wüstite (nonstoichiometric ferrous oxide) powder samples (99\% purity; they were fabricated starting with a higher iron oxide, then reducing the oxide by introducing iron metal) were purchased from Sigma-Aldrich (Sigma-Aldrich Corp., St. Louis, MO). The particle sizes, microstructure, and distribution of the oxides were characterized by a Hitachi S-4700 field-emission scanning electron microscope (FE-SEM, Hitachi Ltd., Tokyo, Japan).

Room temperature X-ray diffraction (RT-XRD) pattern for the sample was obtained using a conventional Scintag XDS2000 powder X-ray diffractometer (Scintag Inc., Cupertino, CA) with a graphite monochromator and $\mathrm{Cu} K_{\alpha}$ radiation. The specific molecular formula of the oxide was determined by calculating the corresponding lattice parameter from the RT-XRD pattern and comparing it with the JCPDS card.

The phase transformation of the sample during the heating was identified by high temperature X-ray diffraction (HT-XRD). The measurements were conducted in vacuum at room temperature $(\mathrm{RT}), 100{ }^{\circ} \mathrm{C}, 200{ }^{\circ} \mathrm{C}, 300{ }^{\circ} \mathrm{C}, 400{ }^{\circ} \mathrm{C}, 500{ }^{\circ} \mathrm{C}, 550{ }^{\circ} \mathrm{C}$, $600{ }^{\circ} \mathrm{C}, 650{ }^{\circ} \mathrm{C}, 700{ }^{\circ} \mathrm{C}, 1000{ }^{\circ} \mathrm{C}$, and $1100{ }^{\circ} \mathrm{C}$ using a PANalytical X'Pert PRO X-ray diffractometer (PANalytical B.V., Almelo, The Netherlands) with $\mathrm{Cu} K_{\alpha}$ radiation. 
The permittivity and permeability of the sample were measured by the cavity perturbation technique. In the permittivity test, ferrous oxide powders passing the 0.1 $\mathrm{mm}$ screen were uniaxially pressed at about $172 \mathrm{MPa}$ in a die lined with tungsten carbide to form 3 pellets with a diameter of about $4.03 \mathrm{~mm}$ and a total, stacked length (height) of $11.7 \mathrm{~mm}$. The bulk density (room temperature) of the sample was $2.43 \mathrm{~g} \mathrm{~cm}^{-}$

${ }^{3}$. During the measurements, the sample was step-heated in the conventional (resistance) furnace to the designated temperatures in $0.01 \mathrm{~L} \mathrm{~min}^{-1}$ flowing argon. The permittivity measurements started at room temperature $\left(\sim 21^{\circ} \mathrm{C}\right)$ and heated in $50^{\circ} \mathrm{C}$ steps to $\sim 1100$ ${ }^{\circ} \mathrm{C}$. In the permeability measurements, the same punch/die unit was used to form pellets with a diameter of about $4.03 \mathrm{~mm}$ and length of $12.6 \mathrm{~mm}$. The bulk density of pellets (room temperature) was $2.59 \mathrm{~g} \mathrm{~cm}^{-3}$. The measurements were done in the same argon, starting at room temperature, then in $25^{\circ} \mathrm{C}$ steps to $550^{\circ} \mathrm{C}$.

The molecular formula of nonstoichiometric ferrous oxide was determined through the RT-XRD pattern (Fig. 5.16), from which the lattice parameter was calculated. The ferrous oxide is identified as $\mathrm{Fe}_{0.925} \mathrm{O}$ (JCPDS card: 89-0686) with the lattice parameter of $4.036 \AA ̊$ using DMSNT (Scintag Inc., Cupertino, CA). 


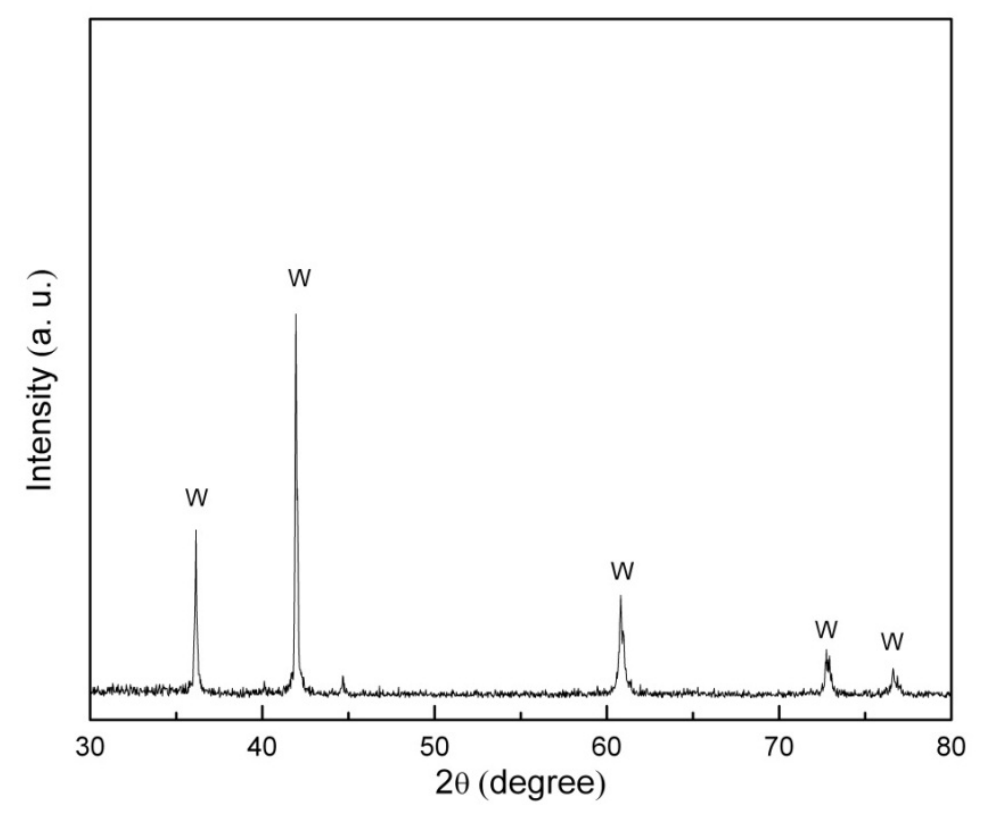

Fig. 5.16. RT-XRD pattern of the sample: $\mathrm{w}-\mathrm{Fe}_{0.925} \mathrm{O}$.

Figure 5.17 presents the measured temperature dependence of permittivity involving the relative dielectric constant and the relative dielectric loss factor $\left(\varepsilon_{r}{ }^{\prime}\right.$ and $\varepsilon_{r}{ }^{\prime \prime}$, respectively) of $\mathrm{Fe}_{0.925} \mathrm{O}$. The permittivity increases slightly along with temperature up to $200{ }^{\circ} \mathrm{C}$, which is possibly associated with promotion of ionic diffusion with increasing temperature. ${ }^{140}$ The FE-SEM image (Fig. 5.18) shows most particles of the sample are distributed in the size range of 0.5 to $1.0 \mu \mathrm{m}$, and accumulate together and form interstices between them. 


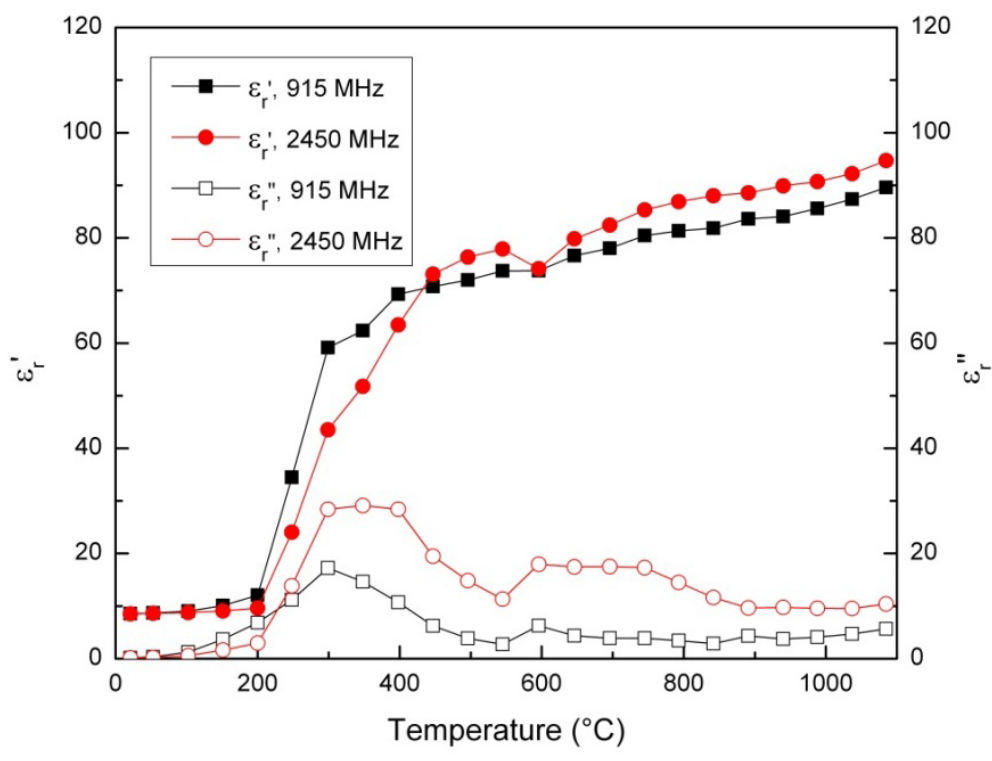

Fig. 5.17. Variation of complex relative permittivity of the sample as a function of temperature.

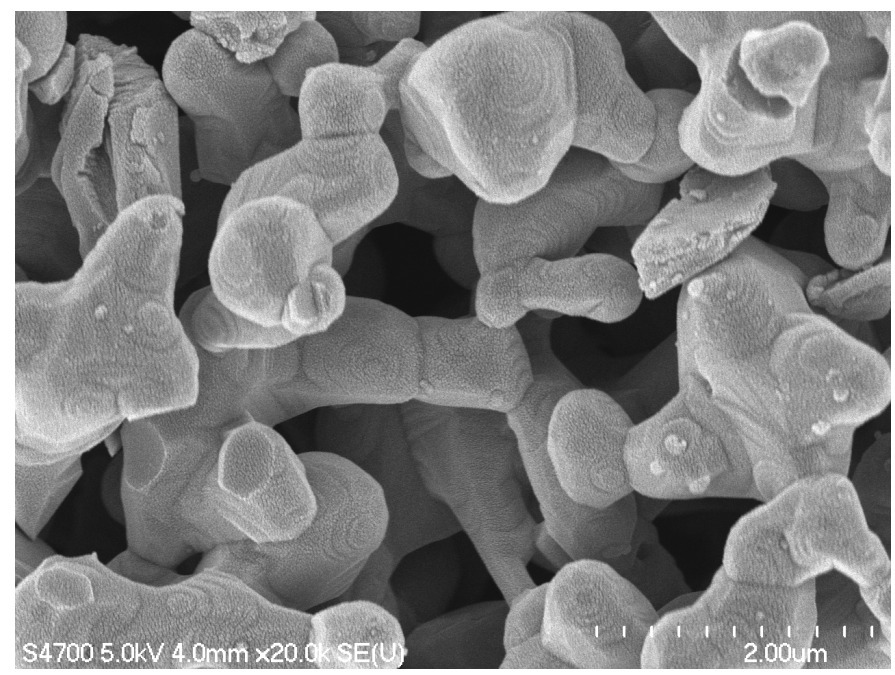

Fig. 5.18. FE-SEM image of $\mathrm{Fe}_{0.925} \mathrm{O}$ particles in 0.5-1.0 $\mu \mathrm{m}$ size accumulating together and forming interstices between them. 
Note that $\varepsilon_{r}{ }^{\prime}$ increases dramatically between 200 and $400{ }^{\circ} \mathrm{C}$, while $\varepsilon_{r}{ }^{\prime \prime}$ exhibits a dielectric loss peak in the range of 200 to $550{ }^{\circ} \mathrm{C}$. This is a typical relaxation/interfacial polarization phenomena behavior, usually indicating a change in the material associated with the loss of an insulating barrier between particles, or the presence of a transient species during a phase change. In this case, it is expected to be mainly associated with the phase transformation resulting from the thermal decomposition of $\mathrm{Fe}_{0.925} \mathrm{O}$, which is clearly proved by the HT-XRD patterns shown in Fig. 5.19. As revealed by the patterns, at temperatures below $200{ }^{\circ} \mathrm{C}$, no phase change occurs. However, in the temperature range of $\sim 300$ to $550{ }^{\circ} \mathrm{C}$, the peaks of magnetite and iron are observed, demonstrating the emergence of thermal decomposition of $\mathrm{Fe}_{0.925} \mathrm{O}$. Thus, the phase change probably contributes to the dramatic change in permittivity between 200 and $550{ }^{\circ} \mathrm{C}$. This observation is in agreement with previous research, which shows the ferrous oxide is thermodynamically unstable below $575^{\circ} \mathrm{C}$, dissociating to metal and $\mathrm{Fe}_{3} \mathrm{O}_{4}{ }^{148-}$ ${ }^{150}$ According to this investigation, the reaction can be expressed as follows:

$$
4 \mathrm{Fe}_{0.925} \mathrm{O} \rightarrow 0.7 \mathrm{Fe}+\mathrm{Fe}_{3} \mathrm{O}_{4}
$$

As temperature continues to increase, the regernation of the ferrous oxide occurs:

$$
0.7 \mathrm{Fe}+\mathrm{Fe}_{3} \mathrm{O}_{4} \rightarrow 4 \mathrm{Fe}_{0.925} \mathrm{O}
$$

As displayed in Fig. 5.19, the HT-XRD patterns of the sample exactly confirm these reactions. The peaks of the magnetite and iron disappear at $600{ }^{\circ} \mathrm{C}$. At even higher temperatures up to $1100{ }^{\circ} \mathrm{C}, \mathrm{Fe}_{0.925} \mathrm{O}$ becomes quite stable. The relative dielectric constant continues to increase in this stage with a much slower rate. The relative 
dielectric loss factor remains almost stable between 600 and $800{ }^{\circ} \mathrm{C}$, and then decreases a little up to $1100^{\circ} \mathrm{C}$. This may be associated with the "sintering effect" occurred during the heating process, as revealed by the FE-SEM microstructure image (Fig. 5.20) of the heated product after the dielectric measurement. In fact, the sintering occurring at temperatures above $1000{ }^{\circ} \mathrm{C}$ makes the particles adhere to each other. During this process densification and grain growth were observed and the decrease in the porosity of the sample and the increase in the average grain size were noticed, which hinders the diffusions of iron and oxygen ions, ${ }^{151}$ possibly influencing the microwave absorption capability in this temperature range.

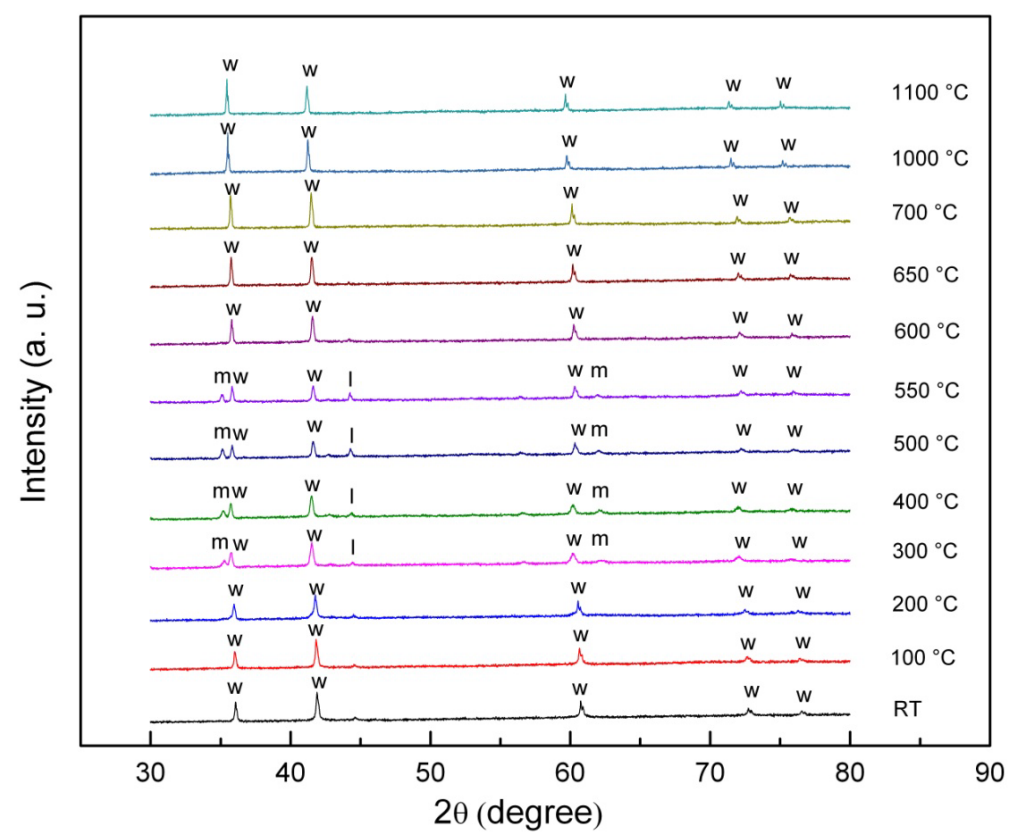

Fig. 5.19. HT-XRD patterns of the sample at various temperatures: $w-\mathrm{Fe}_{0.925} \mathrm{O}$, $\mathrm{m}-\mathrm{Fe}_{3} \mathrm{O}_{4}$, and $\mathrm{I}-\mathrm{Fe}$. 
Figure 5.21 describes the variation of complex relative permeability of the sample as a function of temperature. At the temperature below $\sim 200{ }^{\circ} \mathrm{C}$, the relative magnetic constant and magnetic loss factor $\left(\mu_{r}^{\prime}\right.$ and $\mu_{r}{ }^{\prime \prime}$, respectively) are around 1 and 0 , respectively. It indicates the nonstoichiometric ferrous oxide exhibits little magnetism, which is in agreement with the paramagnetism it exhibits at room temperature. As the temperature increases, the magnetic loss gradually grows likely due to the formation of iron and magnetite from the thermal decomposition of the ferrous oxide, as discussed above. The metal $\mathrm{Fe}$ (ferromagnetic) and $\mathrm{Fe}_{3} \mathrm{O}_{4}$ (ferromagnetic) formed due to the decomposition are expected to contribute to the substantial increase in relative magnetic loss factor. But this trend changes at a temperature of around $550{ }^{\circ} \mathrm{C}$, which is probably related both to the "Curie point" effect where $\mathrm{Fe}_{3} \mathrm{O}_{4}$ loses magnetism, and to the regeneration of $\mathrm{Fe}_{0.925} \mathrm{O} .{ }^{150}$ Thus, the thermal decomposition of $\mathrm{Fe}_{0.925} \mathrm{O}$ hinders the determination of the dielectric and magnetic properties of "true" $\mathrm{Fe}_{0.925} \mathrm{O}$ from $\sim 200$ to $\sim 550{ }^{\circ} \mathrm{C}$. At the temperature higher than $550{ }^{\circ} \mathrm{C}$, however, this "disturbance" can be excluded due to the regeneration of nonstoichiometric ferrous oxide. It also implies a low magnetic loss of the ferrous oxide could be estimated at even higher temperatures $\left(>550{ }^{\circ} \mathrm{C}\right)$ although the experiment did not continue the permeability measurement in this range. Moreover, it should be mentioned that the relative magnetic constant decreases during the decomposition process (above $200{ }^{\circ} \mathrm{C}$ ). This is because the magnetic response of materials depends on the saturation magnetization, which decreases with increasing temperature. ${ }^{152}$ The conversion to magnetite does turn on a ferromagnetic response, but it decreases with increasing temperature eventually. 


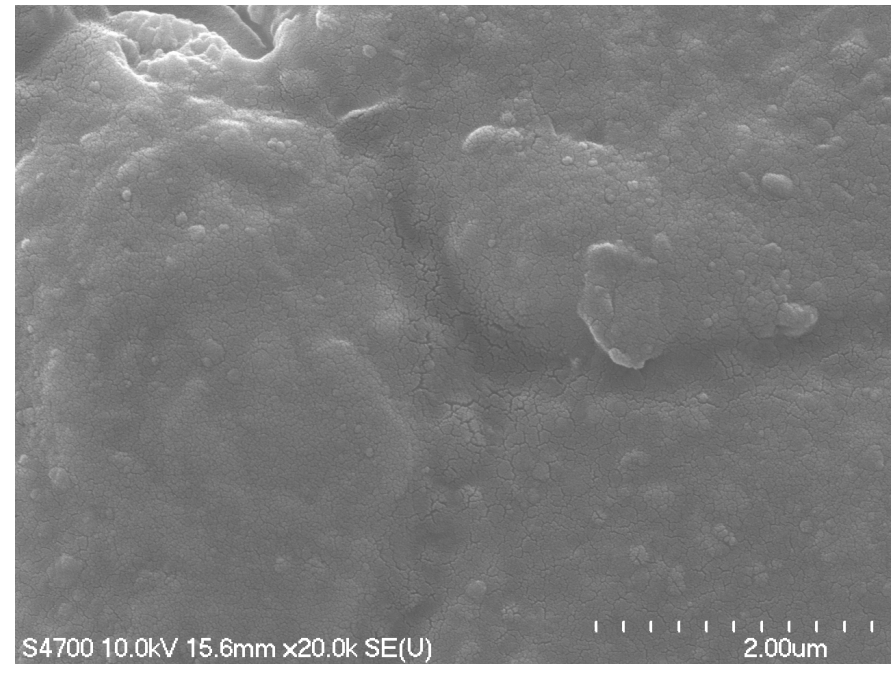

Fig. 5.20. FE-SEM image of $\mathrm{Fe}_{0.925} \mathrm{O}$ after the dielectric measurement.

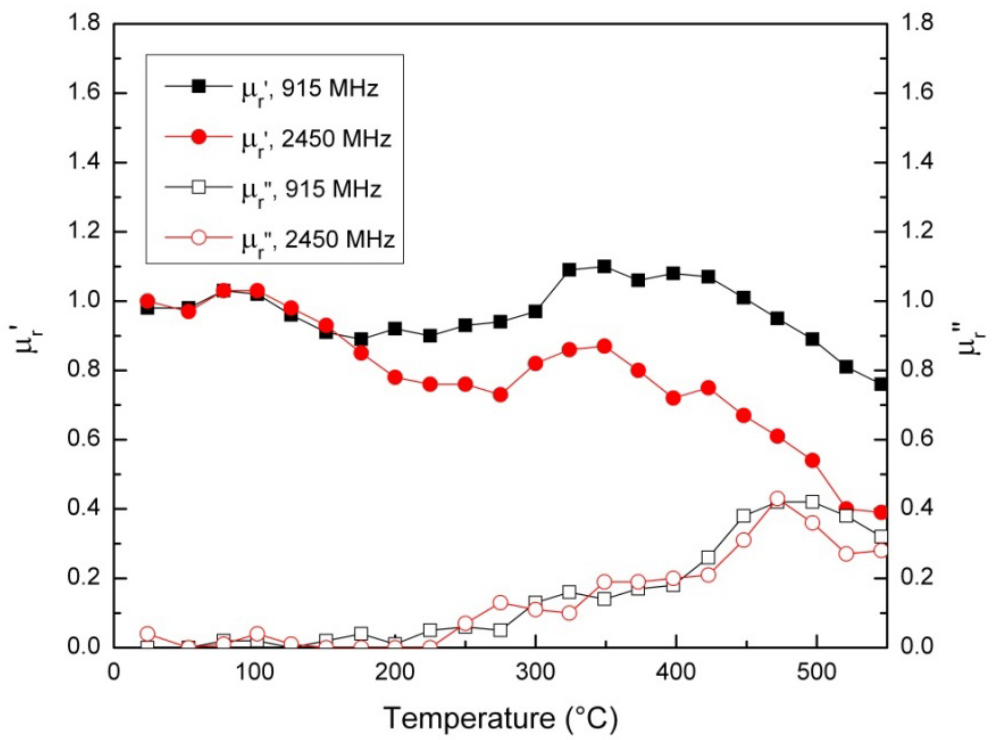

Fig. 5.21. Variation of complex relative permeability of the sample as a function of temperature. 


\subsubsection{Microwave Absorption Capability of Wüstite}

As demonstrated before, the characterization of the microwave propagation behavior of materials should use both the permittivity and permeability to determine the microwave attenuation. Figure 5.22 reveals little difference between the penetration depths calculated by using eqs. (1.5) and (3.22) at low temperatures $\left(20-200{ }^{\circ} \mathrm{C}\right)$. In the low temperature range, the tiny magnetic loss factor of $\mathrm{Fe}_{0.925} \mathrm{O}$ presents little effect on the penetration depth, while the increase in permittivity (actually, the dielectric loss factor, $\left.\varepsilon_{r}{ }^{\prime \prime}\right)$ results in a rapid decrease in penetration depth along with temperature. The small penetration depth (less than $0.03 \mathrm{~m}$ for both frequencies at $200{ }^{\circ} \mathrm{C}$ ) also implies $\mathrm{Fe}_{0.925} \mathrm{O}$ exhibits strong microwave absorption capability at a relatively low temperature. At temperatures between 200 and $550{ }^{\circ} \mathrm{C}$, the phase change due to thermal decomposition of $\mathrm{Fe}_{0.925} \mathrm{O}$ influences the determination of penetration depth of $\mathrm{Fe}_{0.925} \mathrm{O}$, but it still can be expected that the permittivity dominates the microwave absorption of the sample because of much higher complex relative permittivity than the complex relative permeability. This can be further proved by the microwave penetration depth of the sample at the high temperatures $\left(>550^{\circ} \mathrm{C}\right)$, as shown in Fig. 5.23. In this temperature range, it is reasonable to neglect the influence of permeability since the relative permittivity is so high, and the strength of paramagnetism decreases with temperature. It is found that the penetration depth remains almost constant $(\sim 0.11$ and $\sim 0.015 \mathrm{~m}$ at 915 and $2450 \mathrm{MHz}$, respectively) in this range, indicating the quite stable phase composition (actually, pure $\mathrm{Fe}_{0.925} \mathrm{O}$ ) in the measurement, which is in agreement with the HT-XRD 
pattern of the product after the heating. Note that the penetration depth at $915 \mathrm{MHz}$ is relatively longer than that at $2450 \mathrm{MHz}$ mainly due to the apparent difference between the microwave wavelengths $\left(\lambda_{0}\right)$ at these frequencies rather than the frequency dependencies of permittivity and permeability. At both frequencies, the permittivity and permeability show the similar variation trends and close values with increasing temperature. The shallow microwave penetration depths indicate the nonstoichiometric ferrous oxide presents useful microwave absorption capability at high temperatures (550-1100 $\left.{ }^{\circ} \mathrm{C}\right)$.

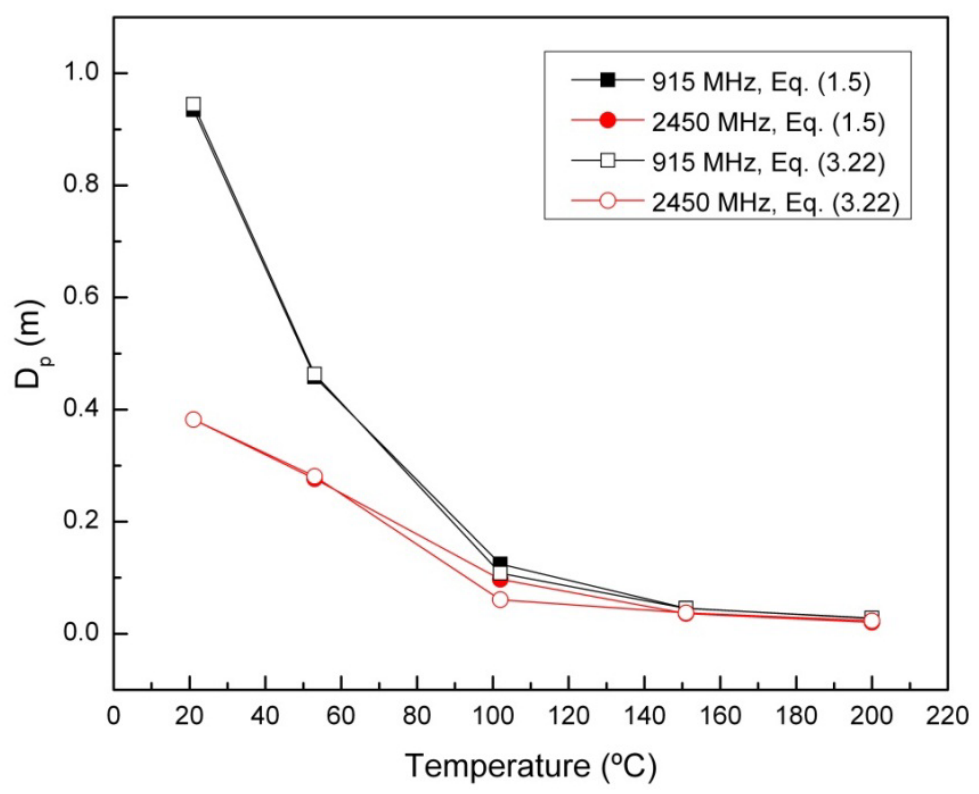

Fig. 5.22. Microwave penetration depth of $\mathrm{Fe}_{0.925} \mathrm{O}$ in the temperature range of 20 to 200 ${ }^{\circ} \mathbf{C}$. 


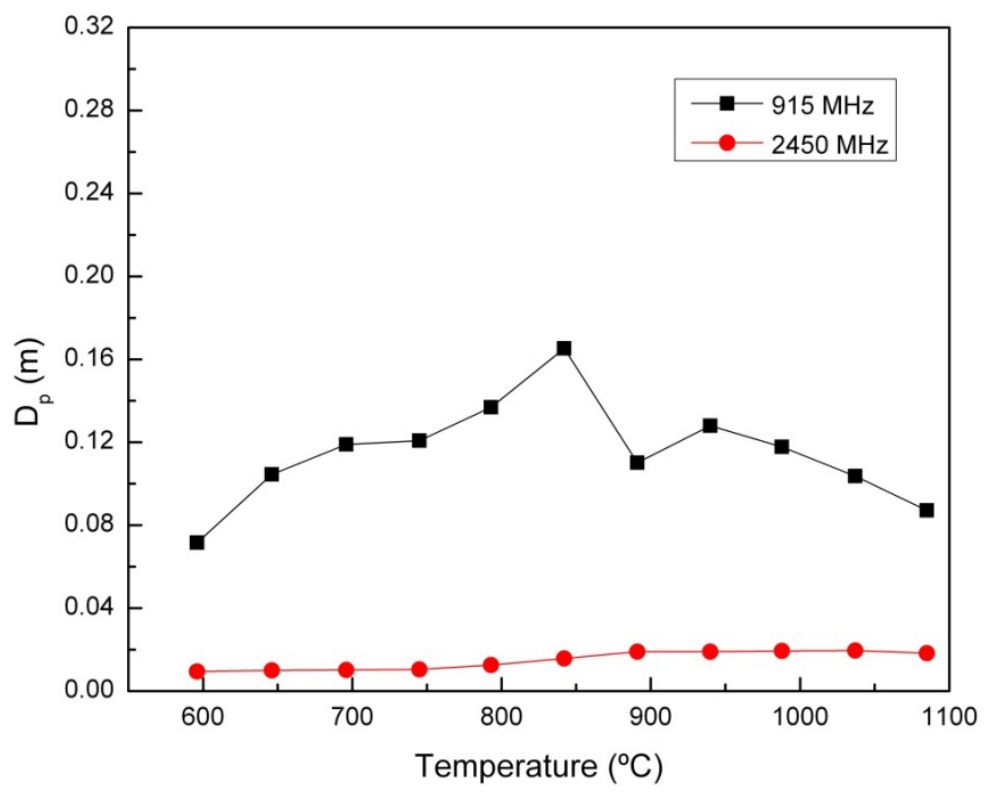

Fig. 5.23. Microwave penetration depth of $\mathrm{Fe}_{0.925} \mathrm{O}$ at temperatures between 550 and 1100 ${ }^{\circ} \mathbf{C}$.

\subsubsection{Microwave Loss of Wüstite}

In the ironmaking process, wüstite begins to form at temperature around $600{ }^{\circ} \mathrm{C}$. Although it decomposes to magnetite and iron at lower temperatures, this thermal behavior does not influence the microwave absorption of wüstite in ironmaking. Thus, only dielectric loss $(2450 \mathrm{MHz}, 1.2 \mathrm{~kW})$ is considered here, as shown in Fig. 5.24. It is found that dielectric loss of wüstite remains relatively stable above $600{ }^{\circ} \mathrm{C}$. 


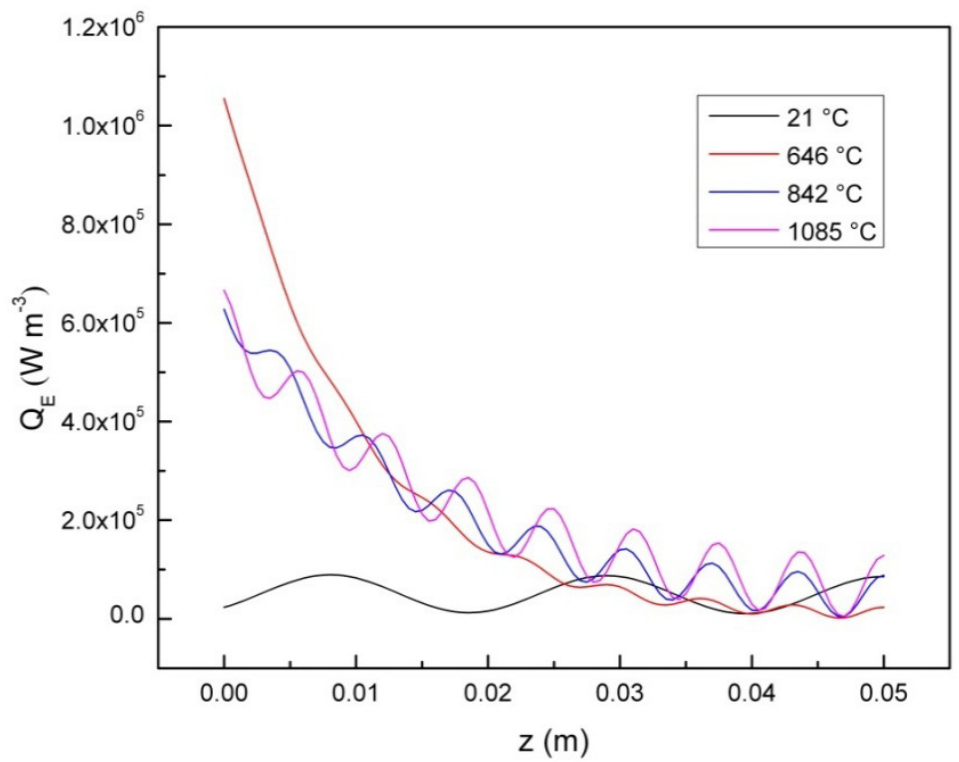

Fig. 5.24. Dielectric loss distributions for microwave heating of the 0.05-m-thick wüstite slabs.

\subsubsection{Kinetics of Wüstite Decomposition}

Nonstoichiometric ferrous oxide (wüstite) is basically an intermediate phase in the ironmaking process. ${ }^{153-155}$ It is also considered as an important transitional material and precursor for various magnetic ceramic/metal nanocompostites or nanocrystals with high values of coercivity for the application as recording media. ${ }^{156-158}$ The substance has been attracting the attention of scientists over a long period. ${ }^{156,159-161}$ Its thermal behavior was investigated by several researchers and a phase transformation due to thermal decomposition of ferrous oxide in a specific temperature range during the heating process was observed. ${ }^{140,148,149}$ Although it has been found that ferrous oxide 
dissociates to magnetite and iron at temperatures lower than $848 \mathrm{~K}$, the decomposition reaction mechanism remains unclear. ${ }^{140}$

The decomposition kinetics has not been well studied mainly due to the product characteristics. Most of investigations on the decomposition kinetics of materials including carbonates, ${ }^{162-166}$ selenites, ${ }^{167}$ permanganates $^{168}$ and metal oxalates ${ }^{169-171}$ are based on the corresponding thermogravimetric measurements. In those studies one product of the decomposition reactions is gas or vapor, leading to the weight loss during the measurements where the decomposition degree (reaction fraction) of reactants can be easily determined. Because the products formed in the decomposition of ferrous oxide are solids, the conventional kinetic analysis relying on the weight change via thermogravimetry (TG) during heating does not work.

In this study, in situ high temperature X-ray diffraction (HT-XRD) was proposed to determine the decomposition degree of ferrous oxide. Then a kinetic function representing the decomposition reaction mechanism of ferrous oxide was determined using the Coats-Redfern method.

The phase transformation of the sample during heating was identified by high temperature X-ray diffraction (HT-XRD), as discussed before. The HT-XRD pattern at each specified temperature was rapidly produced $(<5$ minutes $)$ and collected. The phases presented in the patterns were identified and the decomposition degrees of 
ferrous oxide at various temperatures were calculated through area integrations of the phase peaks.

\section{Theoretical Background}

For a decomposition reaction $\mathrm{A}$ (solid) $\rightarrow \mathrm{B}$ (solid) $+\mathrm{C}$ (solid), the decomposition rate of A can be expressed using the decomposition or conversion degree $(\alpha)$ of A at given time:

$$
\frac{d \boldsymbol{\alpha}}{d t}=k f(\boldsymbol{\alpha})
$$

where $t$ is the reaction time, $k$ is the rate constant and $f(\boldsymbol{\alpha})$ is the conversion function dependent on a specific reaction mechanism. The temperature dependence of the rate constant is normally given by the Arrhenius equation:

$$
k=A^{*} \exp \left(-\frac{E_{a}}{R^{*} T}\right),
$$

where $A^{*}$ is the frequency factor, $E_{a}$ is the activation energy of the decomposition reaction, $R^{*}$ is the gas constant $\left(R^{*}=8.314 \mathrm{~J} \mathrm{~mol}^{-1} \mathrm{~K}^{-1}\right)$ and $T$ is the temperature. With consideration of a constant heating rate $\beta^{*}$, we have

$$
\frac{d T}{d t}=\beta^{*}
$$

Substituting eqs. (5.7) and (5.8) into eq. (5.6) yields the following equation:

$$
\int_{0}^{\boldsymbol{\alpha}} \frac{d \boldsymbol{\alpha}}{f(\boldsymbol{\alpha})}=\frac{A^{*}}{\beta^{*}} \int_{T_{0}}^{T} \exp \left(-\frac{E_{a}}{R^{*} T}\right) d T .
$$


Generally, the left-hand side of eq. (5.9) is denoted with

$$
g(\boldsymbol{\alpha})=\int_{0}^{\boldsymbol{\alpha}} \frac{d \boldsymbol{\alpha}}{f(\boldsymbol{\alpha})}
$$

The integral in the right-hand side of eq. (5.9) does not have an exact analytical solution. Because the value of the integral between 0 and $T_{0}$ is negligible, ${ }^{172}$ the following approximation can be made:

$$
\frac{A^{*}}{\beta^{*}} \int_{T_{0}}^{T} \exp \left(-\frac{E_{a}}{R^{*} T}\right) d T \approx \frac{A^{*}}{\beta^{*}} \int_{0}^{T} \exp \left(-\frac{E_{a}}{R^{*} T}\right) d T .
$$

Further approximation of the right-hand side of eq. (5.11) by applying Cauchy's rule leads to

$$
\frac{A^{*}}{\beta^{*}} \int_{0}^{T} \exp \left(-\frac{E_{a}}{R^{*} T}\right) d T \approx \frac{A^{*} R^{*} T^{2}}{\beta^{*} E_{a}}\left(1-\frac{2 R^{*} T}{E_{a}}\right) \exp \left(-\frac{E_{a}}{R^{*} T}\right) .
$$

The following expression can then be obtained:

$$
\ln \left[\frac{g(\boldsymbol{\alpha})}{T^{2}}\right]=\ln \frac{A^{*} R^{*}}{\beta^{*} E_{a}}\left(1-\frac{2 R^{*} T}{E_{a}}\right)-\frac{E_{a}}{R^{*} T} .
$$

Since $2 R^{*} T / E_{a}<<1$, eq. (5.13) can be simplified as the equation showing the CoatsRedfern relation: ${ }^{173,174}$

$$
\ln \left[\frac{g(\boldsymbol{\alpha})}{T^{2}}\right]=\ln \frac{A^{*} R^{*}}{\beta^{*} E_{a}}-\frac{E_{a}}{R^{*} T}
$$

Thus, a plot of $\ln \left[g(\alpha) / T^{2}\right]$ against $1 / T$ gives the best linear fit representing the most probable decomposition mechanism of the material. The activation energy $\left(E_{a}\right)$ and frequency factor $\left(A^{*}\right)$ can be derived from the slope and intercept of the straight line, 
respectively. As shown in eq. (5.10), the kinetic function of $g(\boldsymbol{\alpha})$ is related to the algebraic expression of function $f(\boldsymbol{\alpha})$. The general formula of $f(\boldsymbol{\alpha})$ is given as ${ }^{175,176}$

$$
f(\boldsymbol{\alpha})=\boldsymbol{\alpha}^{m}(1-\boldsymbol{\alpha})^{n}[-\ln (1-\boldsymbol{\alpha})]^{p},
$$

where $m, n$ and $p$ are the empirical exponent factors and one of them is always zero. The function $g(\boldsymbol{\alpha})$ is obtained through the integration of corresponding expressions of $f(\boldsymbol{\alpha})$, depending on different reaction mechanisms. The most commonly used $f(\boldsymbol{\alpha})$ and $g(\boldsymbol{\alpha})$ corresponding to different kinetic models of the decomposition reaction are summarized in Table 5.3. ${ }^{172,177-179}$

To further understand the decomposition mechanism, other kinetic parameters need to be determined, including enthalpy of activation $\Delta H^{\ddagger}$, entropy of activation $\Delta S^{\ddagger}$, and Gibbs free energy at the temperature of the maximum rate of decomposition $\Delta G_{m}{ }^{\neq}$. They can be calculated using the Eyring-Polanyi equation: ${ }^{180}$

$$
k=\frac{k_{B} T}{h^{*}} \exp \left(\frac{\Delta S^{\neq}}{R^{*}}\right) \exp \left(-\frac{\Delta H^{\neq}}{R^{*} T}\right),
$$

where $k_{B}$ is the Boltzmann constant $\left(k_{B}=1.3806503 \times 10^{-23} \mathrm{~m}^{2} \mathrm{~kg} \mathrm{~s}^{-2} \mathrm{~K}^{-1}\right)$ and $h^{*}$ is the Planck's constant $\left(h=6.6261 \times 10^{-34} \mathrm{~J} \mathrm{~s}\right)$. A linear form of the Eyring-Polanyi equation is given as

$$
\ln \frac{k}{T}=-\frac{\Delta H^{\ddagger}}{R^{*}} \frac{1}{T}+\ln \frac{k_{B}}{h^{*}}+\frac{\Delta S^{\ddagger}}{R^{*}} .
$$

The plot of $\ln (k / T)$ versus $1 / T$ gives a straight line with a slop of $-\Delta H^{\ddagger} / R$, from which the enthalpy of activation $\Delta H^{\ddagger}$ can be derived, and an intercept of $\ln \left(k_{B} / h^{*}\right)+\Delta S^{\ddagger} / R$, from which the entropy of activation $\Delta S^{\neq}$can be calculated. The Gibbs free energy at the 
temperature when decomposition is at its maximum reaction rate can be determined by using the following equation:

$$
\Delta G_{m}^{\neq}=\Delta H^{\neq}-T_{m} \Delta S^{\neq}
$$

where $T_{m}$ is the temperature at which the maximum reaction rate occurs.

Table 5.3

Algebraic expressions of $f(\alpha)$ and $g(\alpha)$ for various kinetic models.

\begin{tabular}{|c|c|c|c|}
\hline Symbol & $f(\boldsymbol{\alpha})$ & $g(\boldsymbol{\alpha})$ & Kinetic models \\
\hline$F_{0}$ & 1 & $\alpha$ & Zero-order kinetics \\
\hline$F_{1 / 2}$ & $(1-\alpha)^{1 / 2}$ & $2\left[1-(1-\boldsymbol{\alpha})^{1 / 2}\right]$ & One-half order kinetics \\
\hline$F_{2 / 3}$ & $(1-\alpha)^{2 / 3}$ & $3\left[1-(1-\boldsymbol{\alpha})^{1 / 3}\right]$ & Two-thirds order kinetics \\
\hline$F_{1}$ & $(1-\alpha)$ & $-\ln (1-\alpha)$ & First-order kinetics \\
\hline$F_{3 / 2}$ & $(1-\alpha)^{3 / 2}$ & $\left.2[1-\alpha)^{-1 / 2}-1\right]$ & Three-halves order kinetics \\
\hline$F_{2}$ & $(1-\alpha)^{2}$ & $\alpha /(1-\alpha)$ & Second-order kinetics \\
\hline$A_{1}$ & $(1-\alpha)$ & $-\ln (1-\alpha)$ & Random nucleation \\
\hline$A_{3 / 2}$ & $(1-\boldsymbol{\alpha})[-\ln (1-\boldsymbol{\alpha})]^{1 / 3}$ & $(3 / 2)[-\ln (1-\alpha)]^{2 / 3}$ & Avrami-Erofeev equation $(\mathrm{n}=1.5)$ \\
\hline$A_{2}$ & $(1-\alpha)[-\ln (1-\alpha)]^{1 / 2}$ & $2[-\ln (1-\boldsymbol{\alpha})]^{1 / 2}$ & Avrami-Erofeev equation $(\mathrm{n}=2)$ \\
\hline$A_{3}$ & $(1-\alpha)[-\ln (1-\alpha)]^{2 / 3}$ & $3[-\ln (1-\boldsymbol{\alpha})]^{1 / 3}$ & Avrami-Erofeev equation $(\mathrm{n}=3)$ \\
\hline$R_{l}$ & $(1-a)^{0}$ & $\alpha$ & $\begin{array}{l}\text { One-dimensional advance of the } \\
\text { reaction interface }\end{array}$ \\
\hline$R_{2}$ & $(1-a)^{1 / 2}$ & $2\left[1-(1-\boldsymbol{\alpha})^{1 / 2}\right]$ & $\begin{array}{l}\text { Two-dimensional advance of the } \\
\text { reaction interface }\end{array}$ \\
\hline$R_{3}$ & $(1-\alpha)^{2 / 3}$ & $3\left[1-(1-\alpha)^{1 / 3}\right]$ & $\begin{array}{l}\text { Three-dimensional advance of the } \\
\text { reaction interface }\end{array}$ \\
\hline$D_{1}$ & $1 / \alpha$ & $\alpha^{2} / 2$ & $\begin{array}{l}\text { One dimensional diffusion or } \\
\text { parabolic law }\left(\boldsymbol{\alpha}=\mathrm{K} t^{1 / 2}\right)\end{array}$ \\
\hline$D_{2}$ & $1 /-\ln (1-\alpha)$ & $(1-\alpha) \ln (1-\alpha)+\alpha$ & $\begin{array}{l}\text { Two dimensional diffusion (Holt- } \\
\text { Cutler-Wadsworth equation) }\end{array}$ \\
\hline$D_{3}$ & $(1-\boldsymbol{\alpha})^{2 / 3} / 1-(1-\boldsymbol{\alpha})^{1 / 3}$ & $3 / 2\left[1-(1-\alpha)^{1 / 3}\right]^{2}$ & $\begin{array}{l}\text { Three dimensional diffusion (Jander } \\
\text { equation) }\end{array}$ \\
\hline$D_{4}$ & $(1-\alpha)^{1 / 3} / 1-(1-\alpha)^{1 / 3}$ & $3 / 2\left[1-2 \alpha / 3-(1-\alpha)^{2 / 3}\right]$ & $\begin{array}{l}\text { Three dimensional diffusion } \\
\text { (Ginstling-Brounshtein equation) }\end{array}$ \\
\hline$D_{5}$ & $(1-\alpha)^{5 / 3} / 1-(1-\alpha)^{1 / 3}$ & $3 / 2\left[(1-\alpha)^{-1 / 3}-1\right]^{2}$ & $\begin{array}{l}\text { Zhuravlev-Lesokhin-Tempelman } \\
\text { equation }\end{array}$ \\
\hline$D_{6}$ & $(1+\boldsymbol{\alpha})^{2 / 3} /(1+\boldsymbol{\alpha})^{1 / 3}-1$ & $3 / 2\left[(1+\alpha)^{1 / 3}-1\right]^{2}$ & $\begin{array}{l}\text { Komatsu-Uemura equation (anti- } \\
\text { Jander equation) }\end{array}$ \\
\hline
\end{tabular}

To study decomposition kinetics of $\mathrm{Fe}_{0.925} \mathrm{O}$, the variation of the decomposition degree with temperature needs to be determined. As demonstrated by the HT-XRD patterns, 
phase change instead of weight loss is observed during the thermal decomposition of the nonstoichiometric ferrous oxide. Decomposition degree derived from traditional TG curves cannot be used and area integrations of peaks for the phases observed in HTXRD patterns may provide a good approximation for the decomposition degree at each temperature. The decomposition degree can be calculated using the following equation:

$$
\boldsymbol{\alpha}=\frac{S_{T}}{S_{T_{0}}},
$$

where $S_{T}$ is the area of ferrous oxide peaks at given temperature and $S_{T 0}$ is the area of the ferrous oxide peaks at room temperature $(294 \mathrm{~K})$. The areas of the peaks were calculated precisely through the function of integration using OriginPro 8.1.

The variation of decomposition degree in the entire temperature range is shown in Fig. 5.25. As shown in this figure, there is only small decomposition when temperature is below $573 \mathrm{~K}$. This result agrees well with the observation from the HT-XRD patterns. In the temperature range of $573-773 \mathrm{~K}$, the decomposition degree increases rapidly with temperature. This is confirmed by the apparent weaker nonstoichimetric ferrous oxide peaks in the patterns. At temperatures higher than $823 \mathrm{~K}$, the degree drops down dramatically corresponding to the absence of peaks of the magnetite and iron. This is, as aforementioned, because of the regeneration of the nonstoichiometric ferrous oxide. 


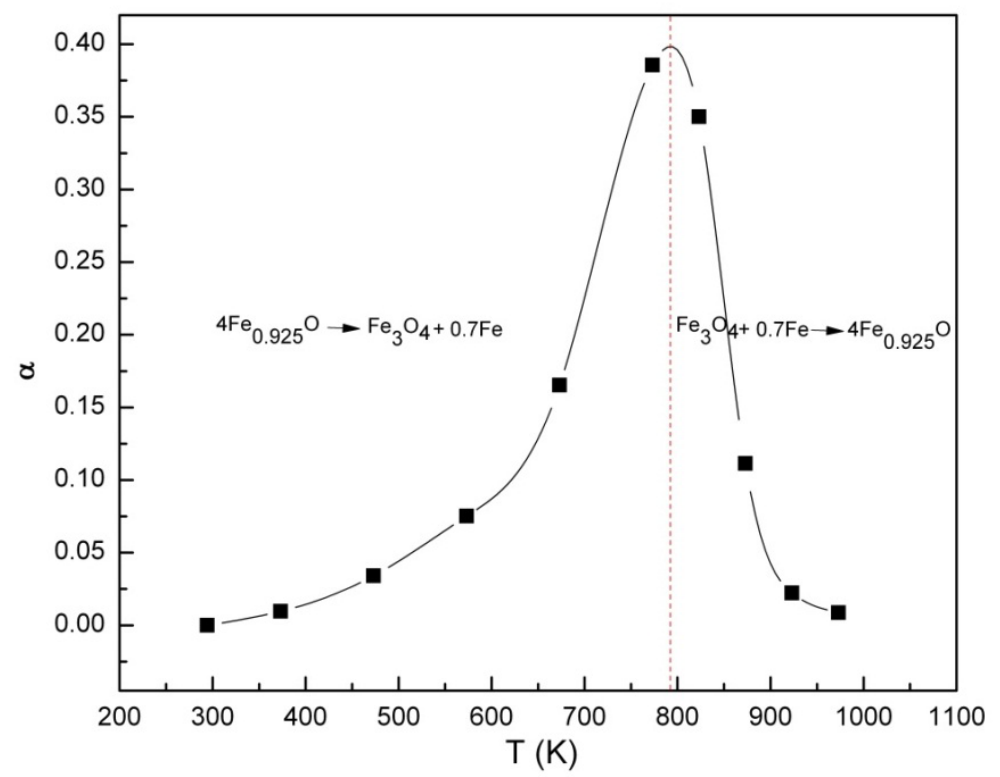

Fig. 5.25. Variation of decomposition degree of $\mathrm{Fe}_{0.925} \mathrm{O}$ with temperature.

For the analysis of kinetics of the decomposition reaction, a reasonable kinetic model needs to be determined. This is dependent on the selection of the algebraic expressions of $f(\boldsymbol{\alpha})$ and $g(\boldsymbol{\alpha})$, from which the reaction mechanism can be estimated. The algebraic expressions of the function $g(\boldsymbol{\alpha})$ listed in Table 5.3 were substituted into eq.(5.14) with the temperatures of the decomposition stage to find the function which would produce the highest correlation coefficient of linear regression $R^{2}$. It is found that the experimental data best delineate a straight line by using the Komatsu-Uemura (antiJander) equation based on a diffusion-controlled mechanism $\left(D_{6}\right)$, as shown in Fig. 5.26. The activation energy and frequency factor were derived from the straight line and other kinetic parameters discussed above were subsequently calculated. The values are listed in Table 5.4 . 


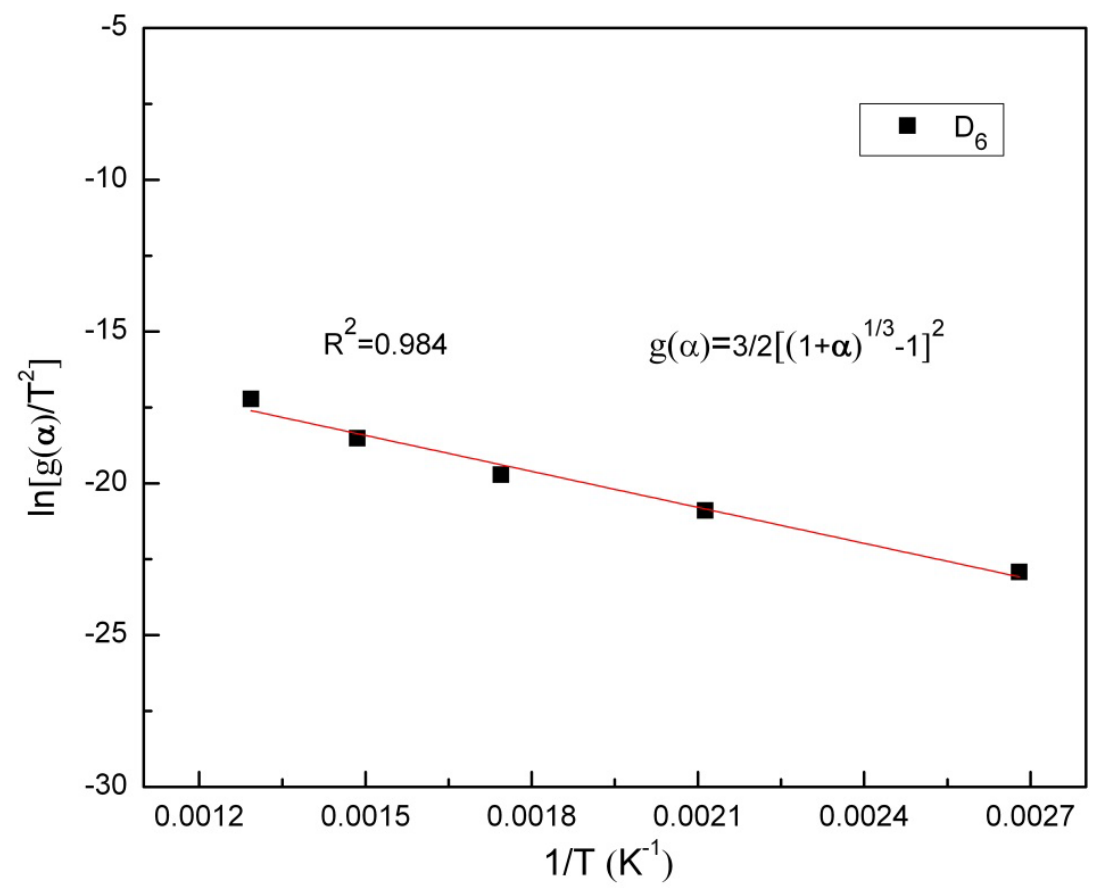

Fig. 5.26. Coats-Redfern plot for the decomposition of $\mathrm{Fe}_{0.925} \mathrm{O}$ using the Komatsu-Uemura equation $\left(D_{6}\right)$.

Table 5.4

Calculated kinetic parameters of the decomposition of $\mathrm{Fe}_{0.925} \mathrm{O}$.

\begin{tabular}{llllll}
\hline Compound & $E_{a} / \mathrm{kJ} \mathrm{mol}^{-1}$ & $A^{*} / \mathrm{min}^{-1}$ & $\Delta H^{\ddagger} / \mathrm{kJ} \mathrm{mol}^{-1}$ & $-\Delta S^{\ddagger} / \mathrm{J} \mathrm{mol}^{-1} \mathrm{~K}^{-1}$ & $\Delta G_{m}^{ \pm} / \mathrm{kJ} \mathrm{mol}^{-1}$ \\
\hline $\mathrm{Fe}_{0.925} \mathrm{O}$ & 32.860 & 0.0594 & 28.537 & 315.651 & 261.535 \\
\hline
\end{tabular}

It is widely accepted that the reactions proceed under diffusion control with activation energy lower than $100 \mathrm{~kJ} \mathrm{~mol}^{-1}$, whereas at higher values they proceed under kinetic control. ${ }^{181}$ As shown in Table 5.4, the decomposition of $\mathrm{Fe}_{0.925} \mathrm{O}$ occurs under diffusion control with activation energy of $32.860 \mathrm{~kJ} \mathrm{~mol}^{-1}$. This indicates that the molecules masses of the reactant and product truly influence the decomposition mechanism. During the decomposition process, the ferrous oxide surface is covered with the incipiently formed magnetite and iron. The diffusion and migration of the formed 
magnetite and iron away from the reactant-product interface control the decomposition rate. Additionally, it can be inferred that the interface between the reactant and product is somewhat steady and the mass transfer through the oxide layer takes place steadily during the decomposition process from the diffusion-controlled model. ${ }^{182}$ The migration rate of $\mathrm{Fe}_{3} \mathrm{O}_{4}$ formed away from the reactant-product interface is relatively slow due to its higher molecular mass and thus strongly affects the reaction kinetics.

In summary, the decomposition kinetics of ferrous oxide was studied under nonisothermal conditions by using HT-XRD. The activation energy and frequency factor of the decomposition reaction were calculated using the Coats-Redfern method and enthalpy of activation $\Delta H^{\ddagger}$, entropy of activation $\Delta S^{\ddagger}$, and Gibbs free energy at the temperature of the maximum rate of decomposition $\Delta{G_{m}}^{\neq}$were determined by the Eyring-Polanyi equation. It is observed that the decomposition reaction of the nonstoichiometric ferrous oxide follows a Komatsu-Uemura model-based diffusion mechanism.

\subsection{Coal}

\subsubsection{Permittivity of Coal}

The sample was an eastern high volatile bituminous coal from West Virginia after drying at $105^{\circ} \mathrm{C}$ for 15 hours in an oven. The proximate analysis (dry basis) shows the 
coal has $9.89 \%$ ash, $30.25 \%$ volatile, $59.86 \%$ fixed carbon and $1.03 \%$ sulfur. In the permittivity test, coal powders with particle size less than $150 \mu \mathrm{m}$ were uniaxially pressed at about $227 \mathrm{MPa}$ in a die lined with tungsten carbide to form 3 pellets with a diameter of about $3.72 \mathrm{~mm}$ and a total, stacked length (height) of $13.08 \mathrm{~mm}$. The bulk density (room temperature) of the sample was $1.11 \mathrm{~g} \mathrm{~cm}^{-3}$.

The cavity perturbation technique was used to determine the dielectric parameters (complex permittivity, ie., relative dielectric constant and loss factor), at 915 and 2450 $\mathrm{MHz}$, the two frequencies available for industrial and domestic applications. During the measurements, the sample was step-heated in the conventional (resistance) furnace to the designated temperatures, starting at $24^{\circ} \mathrm{C}$ and heating in $50{ }^{\circ} \mathrm{C}$ steps (ramp rate: $5{ }^{\circ} \mathrm{C}$ $\min ^{-1}$ ) to $\sim 900{ }^{\circ} \mathrm{C}$, all done in $0.01 \mathrm{~L} \mathrm{~min}^{-1}$ flowing UHP argon.

IR spectra $\left(4000-650 \mathrm{~cm}^{-1}\right)$ of the coal were detected by using a Jasco Fourier Transformed Infrared (FTIR) system, which consists of a FT/IR-4200 spectrometer and an IRT-3000 FTIR microscope.

The phase compositions of the coal during pyrolysis were determined using a Scintag XDS2000 powder X-ray diffractometer (XRD) with a graphite monochromator and $\mathrm{Cu}$ $K_{\alpha}$ radiation. The XRD patterns of the coal at different temperatures were recorded for analysis. 
Under the assumption that the dielectric loss is mainly caused by free electron conductivity, the final state dielectric loss factor was estimated at room temperature by measuring the final direct current (DC) resistance of the sample pellets. The theoretical relationship between the DC resistance and the dielectric loss factor is given by

$$
\varepsilon_{r} "=\frac{1}{2 \cdot \pi \cdot f \cdot \varepsilon_{0} \cdot R \cdot\left(\frac{\pi}{4} \cdot \frac{D^{2}}{l}\right)},
$$

where $f$ is the microwave frequency, $R$ is the resistance measured in the experiment, $D$ is the diameter of the coal pellet, and $L$ is the length of the coal pellet. The DC resistance value was used to calculate a theoretical dielectric loss factor which was then compared with the measured dielectric loss factor as a test of the microwave loss mechanism.

The penetration depth $\left(D_{p}\right)$ is one of the parameters for determining the microwave absorption capability of materials and can be defined as the distance from the surface into the materials at which the traveling wave power drops to $e^{-1}$ from its value at the surface. From the relative dielectric constant and loss factor the penetration depth can be calculated using eq. (1.5).

The temperature dependences of the dielectric parameters are shown in Fig. 5.27. It can be separated into three distinct stages. Up to $500{ }^{\circ} \mathrm{C}$ (stage I), the dielectric constant decreases slightly with increasing temperature. Then a substantial increase in dielectric constant is found in the temperature range of $500-750{ }^{\circ} \mathrm{C}$ (stage II) for both 
frequencies. In the last stage (stage III, Temperature $>750{ }^{\circ} \mathrm{C}$ ), the dielectric constant remains high and relatively stable. The dielectric loss factor behaves similarly, the only difference being the obvious dielectric loss "peak" in the temperature range of 500-750 ${ }^{\circ} \mathrm{C}$ (stage II) shown in Fig. 5.27(c) and Fig. 5.27(d). This is a typical relaxation/interfacial polarization phenomena behavior, usually indicating a change in the material associated with the loss of an insulating barrier. It may also indicate there is a main phase transformation in this temperature range.
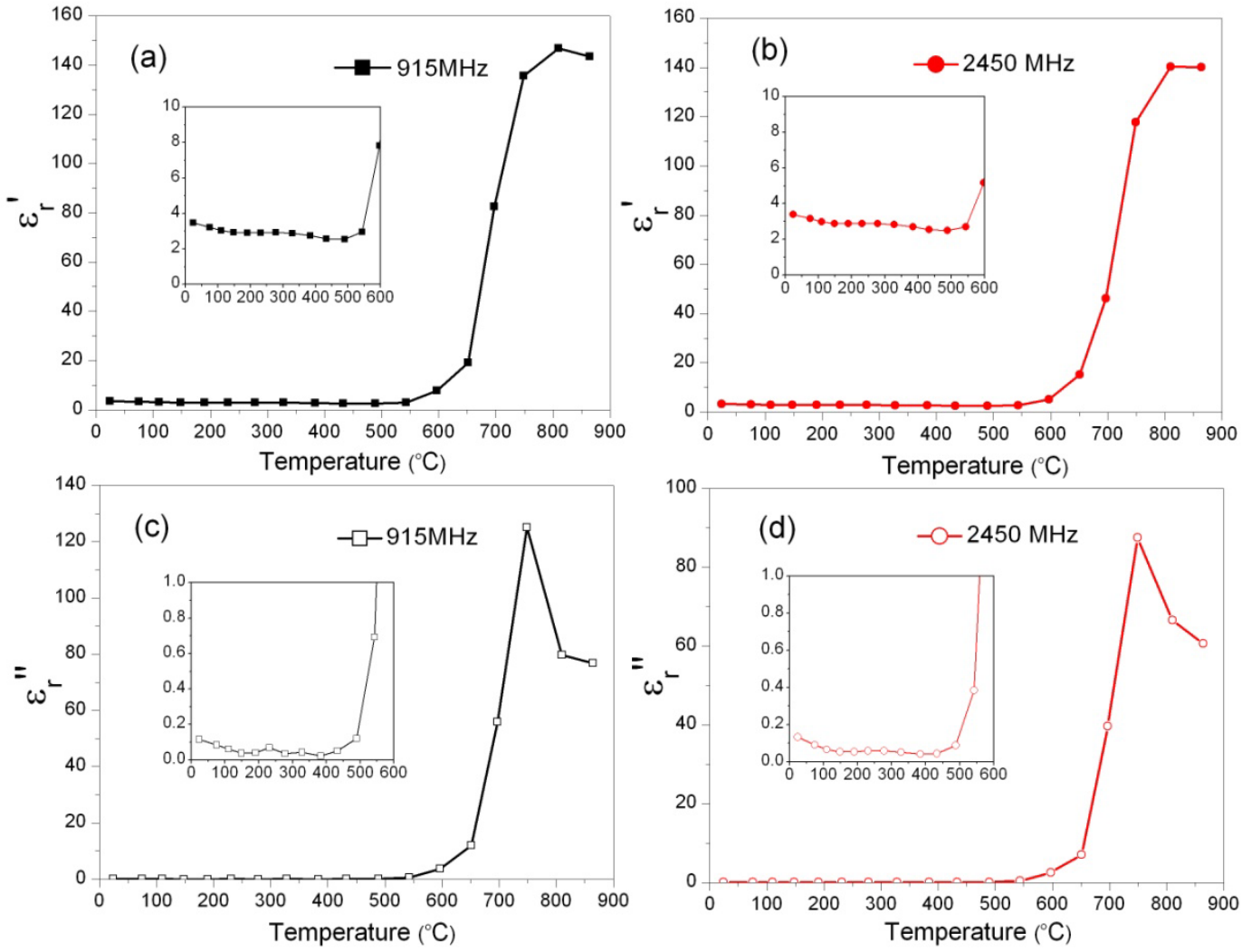

Fig. 5.27. Temperature dependences of dielectric properties of the pressed coal pellets under UHP argon, at 915 and $2450 \mathrm{MHz}$. Insets: magnification patterns as temperature varies from 24 to $600{ }^{\circ} \mathrm{C}$. The initial pellet density is $1.11 \mathrm{~g} \mathrm{~cm}^{-3}$ and the final density is $1.02 \mathrm{~g} \mathrm{~cm}^{-3}$. The mass loss is $43 \%$ of the initial mass (TGA). 
To characterize this phenomenon, the weight change of the coal with temperature during the dielectric measurement was also recorded in the experiment. It is found that about $43 \%$ of the initial mass was lost in stage II, probably due to the loss of the volatile in the coal, especially the organic radicals with high molecular mass. This can also be further characterized by the FTIR spectra of coal shown in Fig. 5.28.

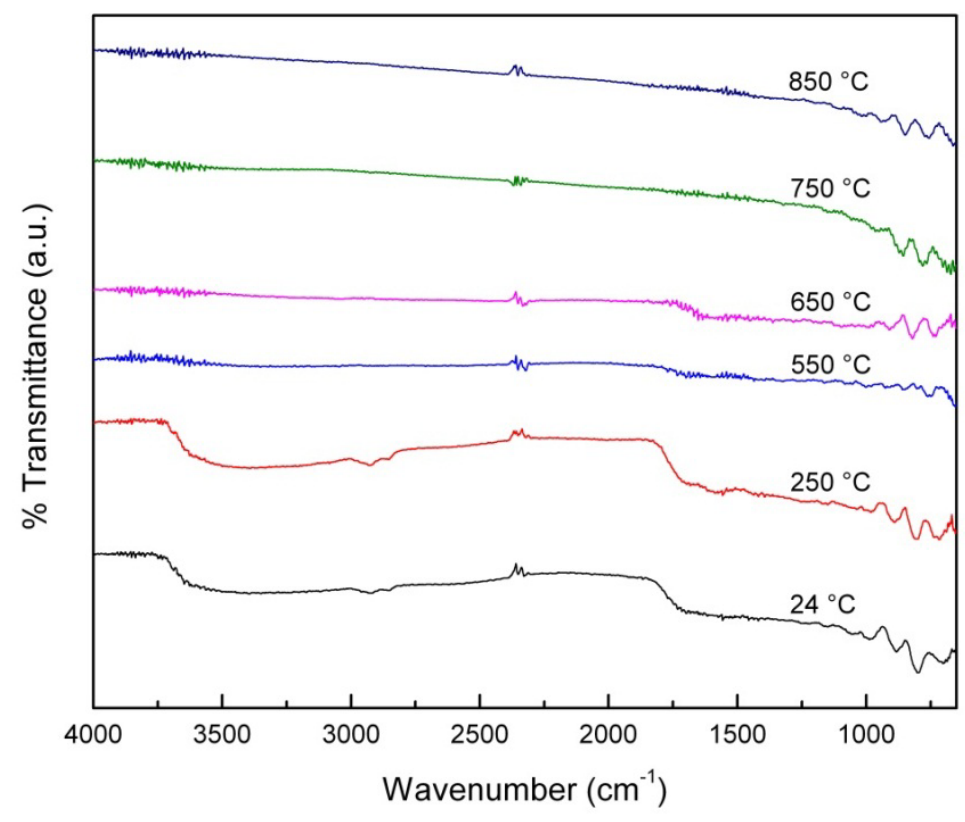

Fig. 5.28. FTIR spectra of the coal at $24^{\circ} \mathrm{C}, 250{ }^{\circ} \mathrm{C}, 550{ }^{\circ} \mathrm{C}, 650{ }^{\circ} \mathrm{C}, 750{ }^{\circ} \mathrm{C}$, and $850{ }^{\circ} \mathrm{C}$.

In the FTIR spectra, a broad absorption band is observed at $3400-3750 \mathrm{~cm}^{-1}$ in the raw coal $\left(24{ }^{\circ} \mathrm{C}\right)$ to be mainly due to O-H groups and a lesser amount of N-H. ${ }^{183,184}$ The small peaks at $2917 \mathrm{~cm}^{-1}$ and $2856 \mathrm{~cm}^{-1}$ may be assigned to aliphatic and alicyclic $\mathrm{CH}_{3}$, $\mathrm{CH}_{2}$ and $\mathrm{CH}$ group (mainly $\mathrm{CH}_{2}$ groups). ${ }^{183}$ Note that the intensity of the peak at 2917 $\mathrm{cm}^{-1}$ is greater than that at $2856 \mathrm{~cm}^{-1}$, indicating the presence of longer aliphatic chains 
in the coal. Also, a strong "abnormal" band at $2360 \mathrm{~cm}^{-1}$ in the coal was observed. This is attributed to the atmospheric $\mathrm{CO}_{2}$ interference during the FTIR measurement. The peak at $1699 \mathrm{~cm}^{-1}$ indicates the presence of carbonyl $(\mathrm{C}=\mathrm{O})$. The peak around $1600 \mathrm{~cm}^{-1}$ (also known as the typical "coal peak") in the coal is observed due to aromatic $\mathrm{C}=\mathrm{C}$, vinylic $\mathrm{C}=\mathrm{C}$ and $\mathrm{O}$-containing functional groups. ${ }^{184}$ Such oxygen-containing functional groups include phenols, alcohols, ethers, carboxylic acid and carbonyls. However, it seems unlikely to differentiate them or identify each oxygen-containing functional group. The absorption peaks at $1005 \mathrm{~cm}^{-1}$ and $1032 \mathrm{~cm}^{-1}$ are caused by the Si-O-Si stretching vibration. The band between $880 \mathrm{~cm}^{-1}$ and $750 \mathrm{~cm}^{-1}$ can be assigned to polycyclic aromatic skeleton. The peak at $702 \mathrm{~cm}^{-1}$ observed in the coal is probably due to aromatic $-\mathrm{CH}_{3}{ }^{183}$

The FTIR spectra in Fig. 5.29 also show the peak variation with temperature. The loss of the $\mathrm{O}-\mathrm{H}$ group at relatively low temperature $\left(\right.$ e.g., $\left.250{ }^{\circ} \mathrm{C}\right)$ is likely what produces the small decreases in dielectric constant and loss factor, as illustrated in Fig. 5.27. The peaks at $2917 \mathrm{~cm}^{-1}$ and $2856 \mathrm{~cm}^{-1}$ also disappear at this temperature indicating the emission of a part of aliphatic $\mathrm{CH}_{2}$ groups. The apparent weight loss in stage II, as stated above, can be assigned to the loss of carbonyl and the typical "coal peak". This broad peak remains until the temperature goes up to $750{ }^{\circ} \mathrm{C}$, as indicated by Fig. 5.29. In other words, $\mathrm{C}=\mathrm{O}$, aromatic $\mathrm{C}=\mathrm{C}$, vinylic $\mathrm{C}=\mathrm{C}$ and $\mathrm{O}$-containing functional groups with high molecular mass are lost in stage II, which is in good agreement with the 
results in the literature, where carbon monoxide is found to be the main emission component in the temperature range of $550-850{ }^{\circ} \mathrm{C} .{ }^{185}$

The Si-O-Si stretching vibration is relatively stable with increasing temperature, which also has been proved by the quartz $\left(\mathrm{SiO}_{2}\right)$ peaks in the XRD patterns (See Fig. 5.30). Besides, the polycyclic aromatic skeleton band between $880 \mathrm{~cm}^{-1}$ and $750 \mathrm{~cm}^{-1}$ and the aromatic $-\mathrm{CH}_{3}$ band at $702 \mathrm{~cm}^{-1}$ are also remained even at $850{ }^{\circ} \mathrm{C}$, indicating a relatively stable aromatic carbon structure during pyrolysis.

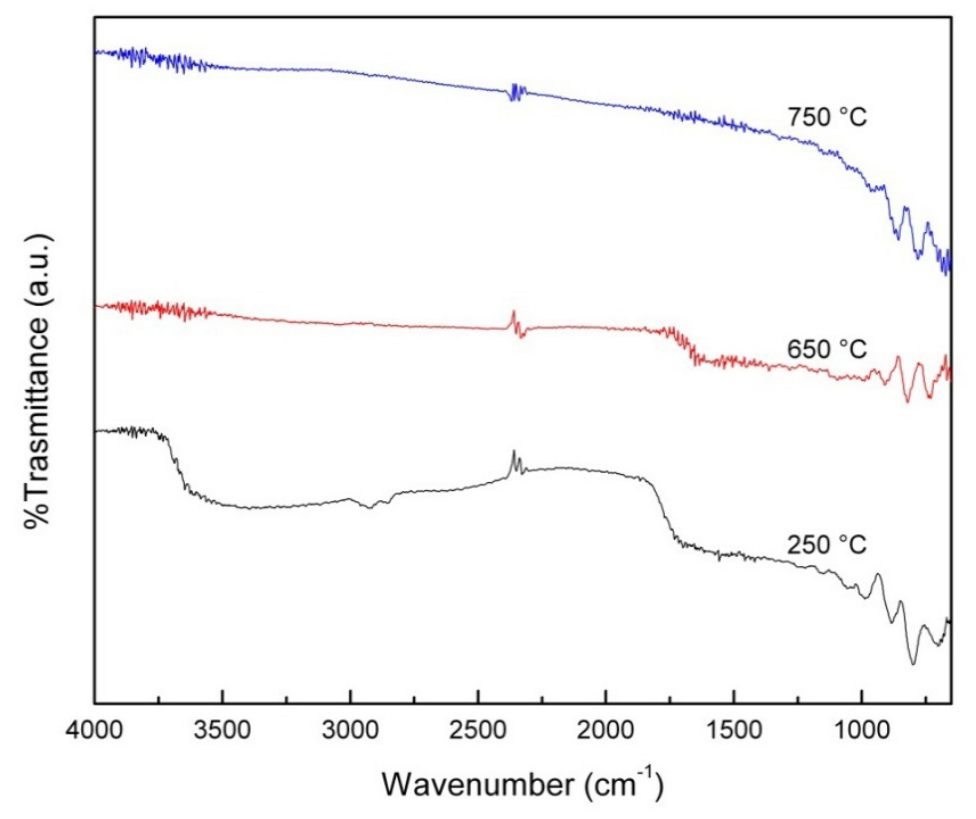

Fig. 5.29. FTIR spectra of the coal at $250{ }^{\circ} \mathrm{C}, 650{ }^{\circ} \mathrm{C}$, and $750{ }^{\circ} \mathrm{C}$. 


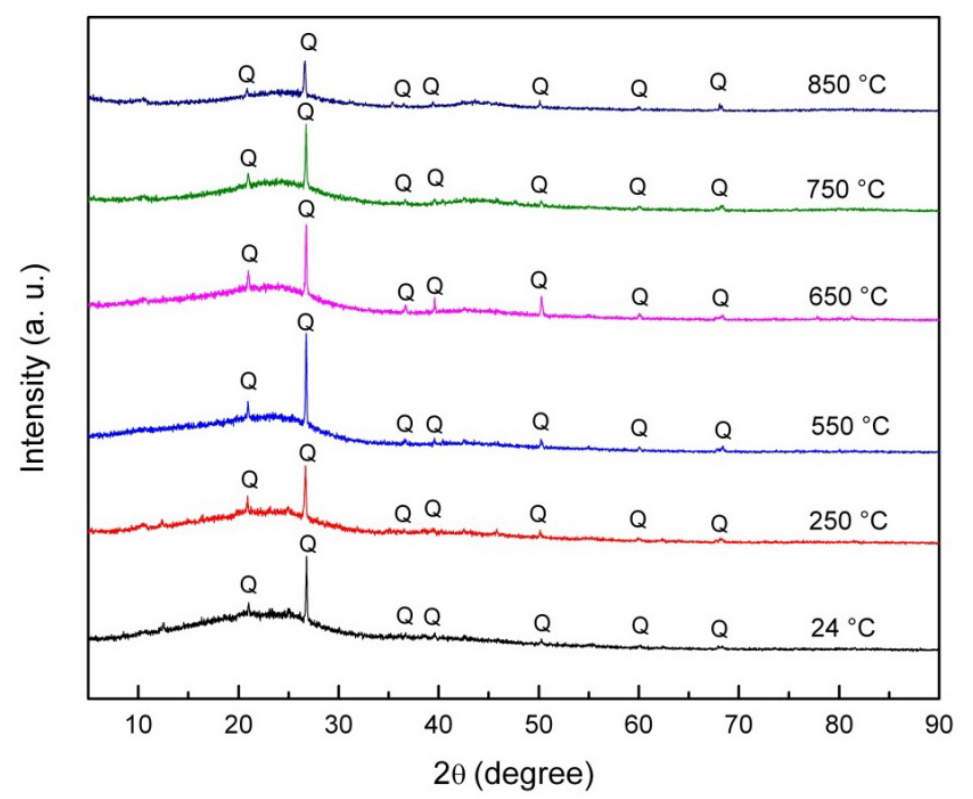

Fig. 5.30. XRD patterns of the coal showing the sharp peaks of quartz $\left(\mathrm{SiO}_{2}\right.$, denoted by "Q").

Because the dielectric loss can be influenced by the conductivity, as indicated by eq. (5.20), a comparison between the calculated and measured (after heating) values of dielectric loss factor may provide further information on the microwave loss mechanism. The DC resistance across the coal pellet after the dielectric measurement at $2450 \mathrm{MHz}$ was $\sim 30 \Omega$. If the microwave loss mechanism is conduction electrons (i.e., normal carbon resistor mechanism), then the value of dielectric loss factor at 2450 $\mathrm{MHz}$, given by eq. (5.20), is $\varepsilon_{r}{ }^{\prime \prime}=32$, very close to the measured value of dielectric loss factor after heating $\left(\varepsilon_{r}{ }^{\prime \prime}=30\right)$. It suggests the microwave loss mechanism for pyrolyzed coal is free electron conduction. 


\subsubsection{Microwave Absorption Capability of Coal}

It is probable that the devolatilization mainly due to the loss of carbonyl $(\mathrm{C}=\mathrm{O})$, aromatic $\mathrm{C}=\mathrm{C}$, vinylic $\mathrm{C}=\mathrm{C}$ and other $\mathrm{O}$-containing functional groups in the coal during pyrolysis leads to the increased conductivity, which produces a dramatic increase in the dielectric loss of the coal in stage II. This also suggests good microwave absorption of the coal can be expected when the temperature exceeds $500{ }^{\circ} \mathrm{C}$. It can be further confirmed by the calculated microwave penetration depths of the coal at 915 and 2450 $\mathrm{MHz}$, as shown in Fig. 5.31. When the temperature goes beyond $500{ }^{\circ} \mathrm{C}$ the penetration depth decreases rapidly. The shallow penetration depths above $750{ }^{\circ} \mathrm{C}$ at both frequencies (approximately 0.008 and $0.003 \mathrm{~m}$ at 915 and $2450 \mathrm{MHz}$, respectively) indicate the excellent microwave absorption capability of the coal at high temperatures.
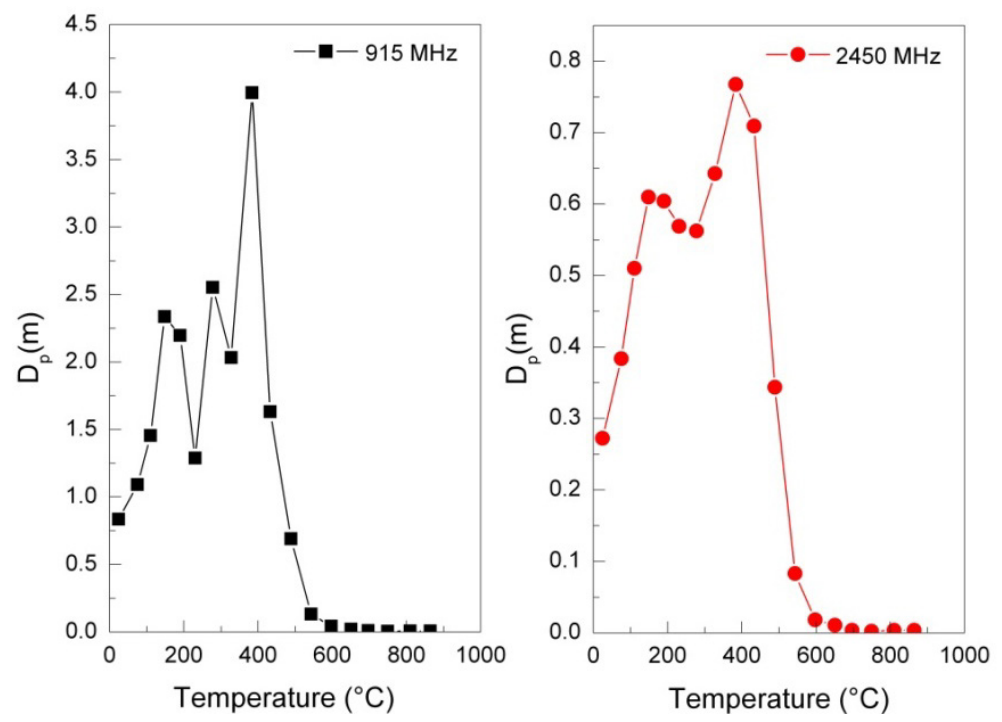

Fig. 5.31. Variations of microwave penetration depth of the coal with temperature at 915 and 2450 MHz. 


\subsubsection{Microwave Loss of Coal}

In general, coal without moisture is a poor microwave absorber at room temperature. The temperature increase of coal is benefited by its microwave dielectric loss. The distribution of dielectric loss for microwave heating $(2450 \mathrm{MHz}, 1.2 \mathrm{~kW})$ of the $0.05-$ m-thick coal slabs is shown in Fig. 5.32. As can be seen from Fig. 5.32, the coal presents strong microwave absorption at high temperatures when a large amount of volatiles are lost.

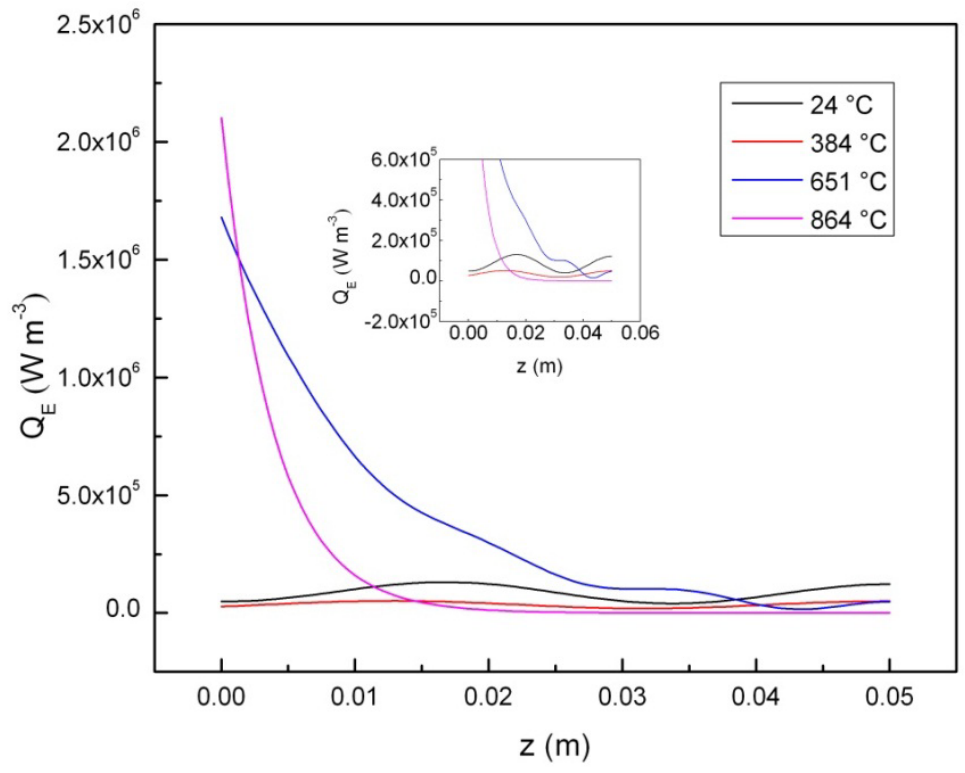

Fig. 5.32. Dielectric loss distributions for microwave heating of the 0.05-m-thick coal slabs. 


\title{
Chapter 6 Microwave Propagation Behaviors in Dielectric
}

\author{
$\operatorname{Media}^{\S}$
}

\subsection{FDTD Method}

The governing differential equations for an electromagnetic field in a general, linear, and isotropic dielectric can be written as ${ }^{187}$

$$
\begin{gathered}
\varepsilon^{\prime} \frac{\partial E}{\partial t}=\nabla \times H-J, \\
\mu^{\prime} \frac{\partial H}{\partial t}=-\nabla \times E-M,
\end{gathered}
$$

where $\varepsilon^{\prime}, \mu^{\prime}, E$, and $H$ are the real part of complex permittivity, real part of complex permeability, electric field strength, and magnetic field strength, respectively.

$J$ is the electric current density and $M$ is the magnetic current density. They can be written as

$$
J=\sigma \cdot E
$$

\footnotetext{
$\S$ The content of this chapter was previously published in TMS Annual Meeting, Extraction and Processing Division - 2012 EPD Congress - Held During the TMS 2012 Annual Meeting and Exhibition ${ }^{186}$ by Zhiwei Peng, Jiann-Yang Hwang, Matthew Andriese, Zheng Zhang, Xiaodi Huang.

Reproduced with permission from TMS Annual Meeting, Extraction and Processing Division - 2012 EPD Congress - Held During the TMS 2012 Annual Meeting and Exhibition: Zhiwei Peng, Jiann-Yang Hwang, Matthew Andriese, Zheng Zhang, Xiaodi Huang. Heat Transfer Characteristics of Magnetite under Microwave Irradiation. TMS Annual Meeting, Extraction and Processing Division - 2012 EPD Congress - Held During the TMS 2012 Annual Meeting and Exhibition. 2012;121-128. Copyright (C) 2012 The Minerals, Metals and Materials Society. (See Appendix D-1 for copyright permission).
} 


$$
M=\sigma^{*} \cdot H,
$$

where $\sigma$ and $\sigma^{*}$ are the electric conductivity and equivalent magnetic loss, respectively.

Expansion of the vector components of the curl operators in the above equations yields the following system of six coupled scalar equations under Cartesian coordinates:

$$
\begin{gathered}
\frac{\partial E_{x}}{\partial t}=\frac{1}{\varepsilon^{\prime}}\left(\frac{\partial H_{z}}{\partial y}-\frac{\partial H_{y}}{\partial z}\right)-\frac{\sigma}{\varepsilon^{\prime}} E_{x}, \\
\frac{\partial E_{y}}{\partial t}=\frac{1}{\varepsilon^{\prime}}\left(\frac{\partial H_{x}}{\partial z}-\frac{\partial H_{z}}{\partial x}\right)-\frac{\sigma}{\varepsilon^{\prime}} E_{y}, \\
\frac{\partial E_{z}}{\partial t}=\frac{1}{\varepsilon^{\prime}}\left(\frac{\partial H_{y}}{\partial x}-\frac{\partial H_{x}}{\partial y}\right)-\frac{\sigma}{\varepsilon^{\prime}} E_{z}, \\
\frac{\partial H_{x}}{\partial t}=-\frac{1}{\mu^{\prime}}\left(\frac{\partial E_{z}}{\partial y}-\frac{\partial E_{y}}{\partial z}\right)-\frac{\sigma^{*}}{\mu^{\prime}} H_{x}, \\
\frac{\partial H_{z}}{\partial t}=-\frac{1}{\mu^{\prime}}\left(\frac{\partial E_{x}}{\partial z}-\frac{\partial E_{z}}{\partial x}\right)-\frac{\sigma^{*}}{\mu^{\prime}} H_{y}, \\
\left.\frac{\partial E_{y}}{\partial x}-\frac{\partial E_{x}}{\partial y}\right)-\frac{\sigma^{*}}{\mu^{\prime}} H_{z} .
\end{gathered}
$$

The system of six coupled partial differential eqs. (6.5)-(6.10) forms the basis of the FDTD numerical algorithm for modeling electromagnetic wave interactions with arbitrary three-dimensional objects. 
Yee's FDTD scheme (see Fig. 6.1) discretizes Maxwell's curl equations by approximating the time and space first-order partial derivatives with central differences, and then solving the resulting equations by using a leapfrog scheme. ${ }^{114}$

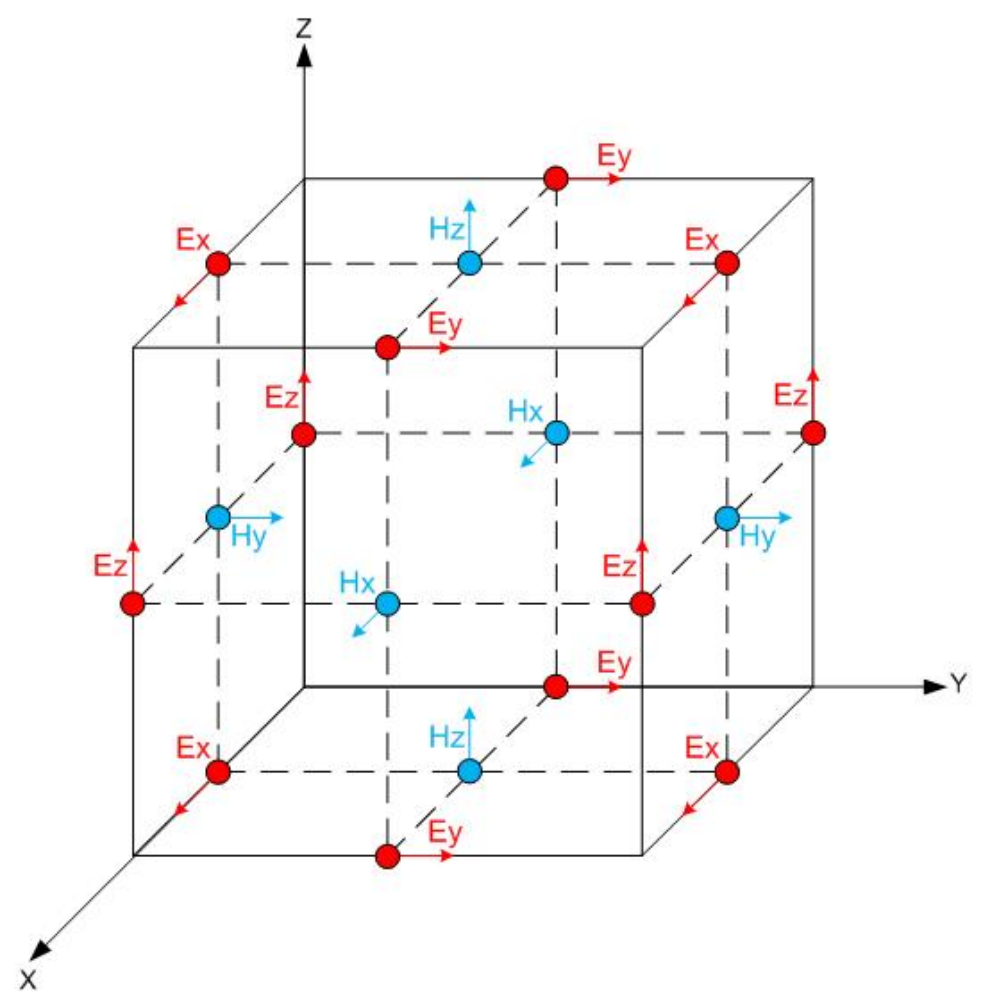

Fig. 6.1. Yee cell in FDTD method.

\subsection{Formulations of the FDTD Algorithm}

Since $\varepsilon_{0}$ and $\mu_{0}$ differ by several orders of magnitude, $E$ and $H$ will differ by several orders of magnitude, too. To simplify the computation result, the electric field strength is normalized: ${ }^{188}$ 


$$
\tilde{E}=\sqrt{\frac{\varepsilon_{r}{ }^{\prime} \varepsilon_{0}}{\mu_{r}{ }^{\prime} \mu_{0}}} E .
$$

Considering one-dimensional equation, we have

$$
\begin{gathered}
\frac{\partial E_{x}(t)}{\partial t}=-\frac{1}{\varepsilon_{r}{ }^{\prime} \varepsilon_{0}} \frac{\partial H_{y}(t)}{\partial z}-\frac{\sigma}{\varepsilon_{r}{ }^{\prime} \varepsilon_{0}} E_{x}(t), \\
\frac{\partial H_{y}(t)}{\partial t}=-\frac{1}{\mu_{r}{ }^{\prime} \mu_{0}} \frac{\partial E_{x}(t)}{\partial z}-\frac{\sigma^{*}}{\mu_{r}{ }^{\prime} \mu_{0}} H_{y}(t), \\
\frac{\tilde{E}_{x}^{n+\frac{1}{2}}(k)-\tilde{E}_{x}^{n-\frac{1}{2}}(k)}{\Delta t}= \\
\frac{1}{\varepsilon_{r}{ }^{\prime} \sqrt{\varepsilon_{0} \mu_{0}}} \frac{H_{y}^{n}\left(k+\frac{1}{2}\right)-H_{y}^{n}\left(k-\frac{1}{2}\right)}{\Delta z}-\frac{\sigma}{\varepsilon_{r}{ }^{\prime} \varepsilon_{0}} \frac{\tilde{E}_{x}^{n+\frac{1}{2}}(k)+\tilde{E}_{x}^{n-\frac{1}{2}}(k)}{2}, \\
\frac{H_{y}^{n+1}\left(k+\frac{1}{2}\right)-H_{y}^{n}\left(k+\frac{1}{2}\right)}{\Delta t}= \\
-\frac{1}{\mu_{r}{ }^{\prime} \sqrt{\varepsilon_{0} \mu_{0}}} \frac{\tilde{E}_{y}^{n+\frac{1}{2}}(k+1)-\tilde{E}_{x}^{n+\frac{1}{2}}(k)}{\Delta z}-\frac{\sigma^{*}}{\mu_{r}{ }^{\prime} \mu_{0}} \frac{H_{y}^{n+1}\left(k+\frac{1}{2}\right)+H_{y}^{n}\left(k+\frac{1}{2}\right)}{2} .
\end{gathered}
$$

To simplify eqs. (6.14) and (6.15), we apply the following equation:

$$
\frac{1}{\sqrt{\varepsilon_{0} \mu_{0}}} \frac{\Delta t}{\Delta x}=\frac{1}{2} .
$$

Then, eq. (6.14) becomes 


$$
\tilde{E}_{x}^{n+\frac{1}{2}}(k)\left[1+\left(\frac{\Delta t \cdot \sigma}{2 \varepsilon_{r}{ }^{\prime} \varepsilon_{0}}\right)\right]=\tilde{E}_{x}^{n-\frac{1}{2}}(k)\left[1-\left(\frac{\Delta t \cdot \sigma}{2 \varepsilon_{r}{ }^{\prime} \varepsilon_{0}}\right)\right]-\frac{\frac{1}{2}}{\varepsilon_{r}{ }^{\prime}}\left[H_{y}^{n}\left(k+\frac{1}{2}\right)-H_{y}^{n}\left(k-\frac{1}{2}\right)\right],
$$

or

$$
\tilde{E}_{x}^{n+\frac{1}{2}}(k)=\tilde{E}_{x}^{n-\frac{1}{2}}(k)\left[\frac{1-\left(\frac{\Delta t \cdot \sigma}{2 \varepsilon_{r}{ }^{\prime} \varepsilon_{0}}\right)}{1+\left(\frac{\Delta t \cdot \sigma}{2 \varepsilon_{r}{ }^{\prime} \varepsilon_{0}}\right)}\right]-\frac{\frac{1}{2}}{\varepsilon_{r}{ }^{\prime} \cdot\left[1+\left(\frac{\Delta t \cdot \sigma}{2 \varepsilon_{r}{ }^{\prime} \varepsilon_{0}}\right)\right]}\left[H_{y}^{n}\left(k+\frac{1}{2}\right)-H_{y}^{n}\left(k-\frac{1}{2}\right)\right] .
$$

Equation (6.15) becomes

$$
\begin{gathered}
H_{y}^{n+1}\left(k+\frac{1}{2}\right)\left[1+\left(\frac{\Delta t \cdot \sigma^{*}}{2 \mu_{r}{ }^{\prime} \mu_{0}}\right)\right]= \\
H_{y}^{n}\left(k+\frac{1}{2}\right)\left[1-\left(\frac{\Delta t \cdot \sigma^{*}}{2 \mu_{r} \mu_{0}}\right)\right]-\frac{\frac{1}{2}}{\mu_{r}^{\prime}}\left[\tilde{E}_{x}^{n+\frac{1}{2}}(k+1)-\tilde{E}_{x}^{n+\frac{1}{2}}(k)\right], \\
H_{y}^{n+1}\left(k+\frac{1}{2}\right)= \\
H_{y}^{n}\left(k+\frac{1}{2}\right)\left[\frac{1-\left(\frac{\Delta t \cdot \sigma^{*}}{2 \mu_{r}^{\prime} \mu_{0}}\right)}{1+\left(\frac{\Delta t \cdot \sigma^{*}}{2 \mu_{r} \mu_{0}}\right)}\right]-\frac{\frac{1}{2}}{\mu_{r}^{\prime} \cdot\left[1+\left(\frac{\Delta t \cdot \sigma^{*}}{2 \mu_{r}^{\prime} \mu_{0}}\right)\right]}\left[\tilde{E}_{x}^{n+\frac{1}{2}}(k+1)-\tilde{E}_{x}^{n+\frac{1}{2}}(k)\right] .
\end{gathered}
$$

Thus, the following equations can be obtained to perform the computation simulation:

$$
\begin{aligned}
& \operatorname{ex}[k]=c a[k] \cdot \operatorname{ex}[k]+c b[k] \cdot(h y[k-1]-h y[k]), \\
& h y[k]=c c[k] \cdot h y[k]+c d[k] \cdot(\operatorname{ex}[k]-\operatorname{ex}[k+1]),
\end{aligned}
$$

where 


$$
\begin{gathered}
c a[k]=\frac{1-\left(\frac{\Delta t \cdot \sigma}{2 \varepsilon_{r}{ }^{\prime} \varepsilon_{0}}\right)}{1+\left(\frac{\Delta t \cdot \sigma}{2 \varepsilon_{r}{ }^{\prime} \varepsilon_{0}}\right)}, \\
c b[k]=\frac{\frac{1}{2}}{\varepsilon_{r}{ }^{\prime} \cdot\left[1+\left(\frac{\Delta t \cdot \sigma}{2 \varepsilon_{r}{ }^{\prime} \varepsilon_{0}}\right)\right]}, \\
c c[k]=\frac{1-\left(\frac{\Delta t \cdot \sigma^{*}}{2 \mu_{r}{ }^{\prime} \mu_{0}}\right)}{1+\left(\frac{\Delta t \cdot \sigma^{*}}{2 \mu_{r}{ }^{\prime} \mu_{0}}\right)}, \\
c d[k]=\frac{\frac{1}{2}}{\mu_{r}{ }^{\prime} \cdot\left[1+\left(\frac{\Delta t \cdot \sigma^{*}}{2 \mu_{r}{ }^{\prime} \mu_{0}}\right)\right]} .
\end{gathered}
$$

\subsection{Modeling of Microwave Propagation in Various Media}

This work simulated the microwave propagation in several typical media, which were characterized by different permittivity, permeability, conductivity and equivalent magnetic loss. The modeling geometry is shown in Fig. 6.2. As shown in the figure, the whole space domain $(1 \mathrm{~m})$ was divided into two equal parts. The left half domain was specified as free space, where the pulse source $(2450 \mathrm{MHz})$ was excited at the cell position of 20. The right half domain was a given medium with varying dielectric and magnetic properties. Note that there were absorbing boundary conditions at both ends of 
the space domain, which guaranteed no microwaves reflection occurring in the simulation area. In the modeling, the pulse source ( $E$ field) was given by

$$
\text { pulse }=E_{x}=\sin \left(2 \pi \cdot f \cdot \frac{\Delta z}{2 \times 3 \times 10^{8}} \cdot N\right)
$$

where $N$ is the computation steps (used for counting the propagation time), and $\Delta z$ is the space step in the calculation $(0.00125 \mathrm{~m})$.

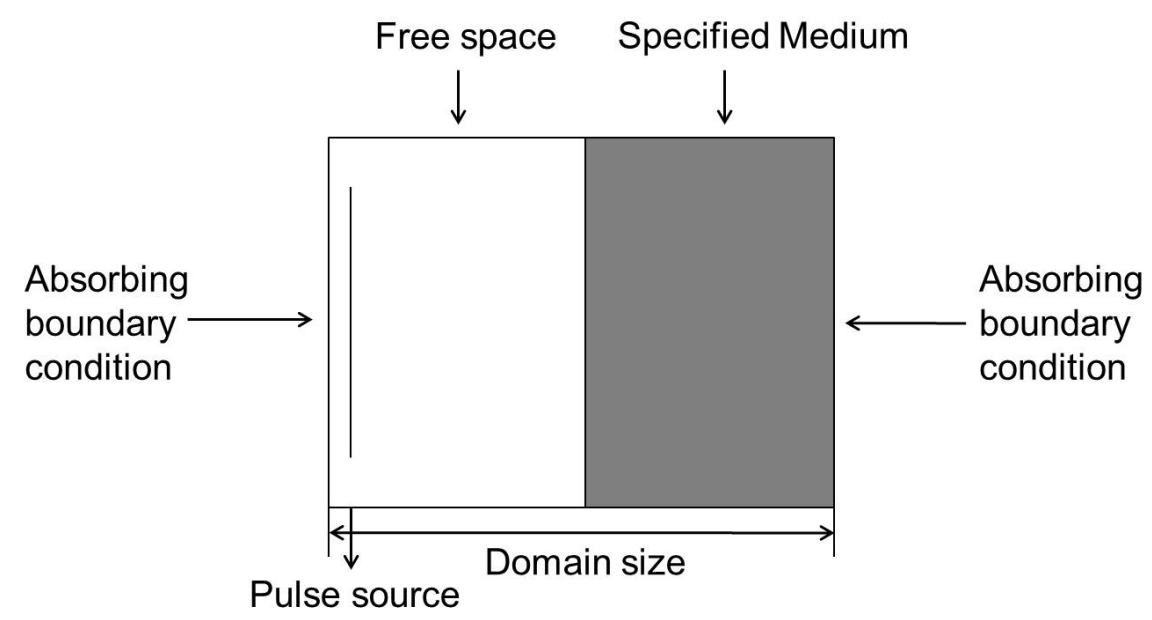

Fig. 6.2. Geometry of space domain (800 cells) in the simulation.

The distributions of microwave fields ( $E$ and $H$ fields) in the space domain after the given computation steps for different media (free space, metal, non-lossy dielectric medium, lossy dielectric medium, magnetic dielectric without magnetic loss, and magnetic dielectric with magnetic loss) are shown in Figs. 6.3-6.16. As shown in these figures, there is no dissipation of $E$ or $H$ fields in free space $\left(\varepsilon_{r}{ }^{\prime}=1, \varepsilon_{r}{ }^{\prime \prime}=0\right)$, while all microwaves are reflected by the metal $\left(\sigma=1 \times 10^{7} \mathrm{~S} \mathrm{~m}^{-1}, \mathrm{Fe}\right)$. As for the non-lossy dielectric medium, the magnitude of $E$ field decreases due to the real part of permittivity 
$\left(\varepsilon_{r}^{\prime}=4, \varepsilon_{r}{ }^{\prime \prime}=0\right)$, giving rise to energy stored in the material. For microwave lossy materials, microwave fields decay rapidly in the lossy dielectric medium $\left(\varepsilon_{r}=4-0.3 \mathrm{j}, \mu_{r}\right.$ $=1)$, the magnetic dielectric without magnetic loss $\left(\varepsilon_{r}=4-0.3 \mathrm{j}, \mu_{r}=1.5\right)$ and the magnetic dielectric with magnetic loss $\left(\varepsilon_{r}=4-0.3 \mathrm{j}, \mu_{r}=1.5-0.2 \mathrm{j}\right)$. Also, it is observed that the fields reduce much faster in the magnetic dielectrics than in other media owing to the contribution of magnetic response under microwave irradiation.

\subsubsection{Free Space}

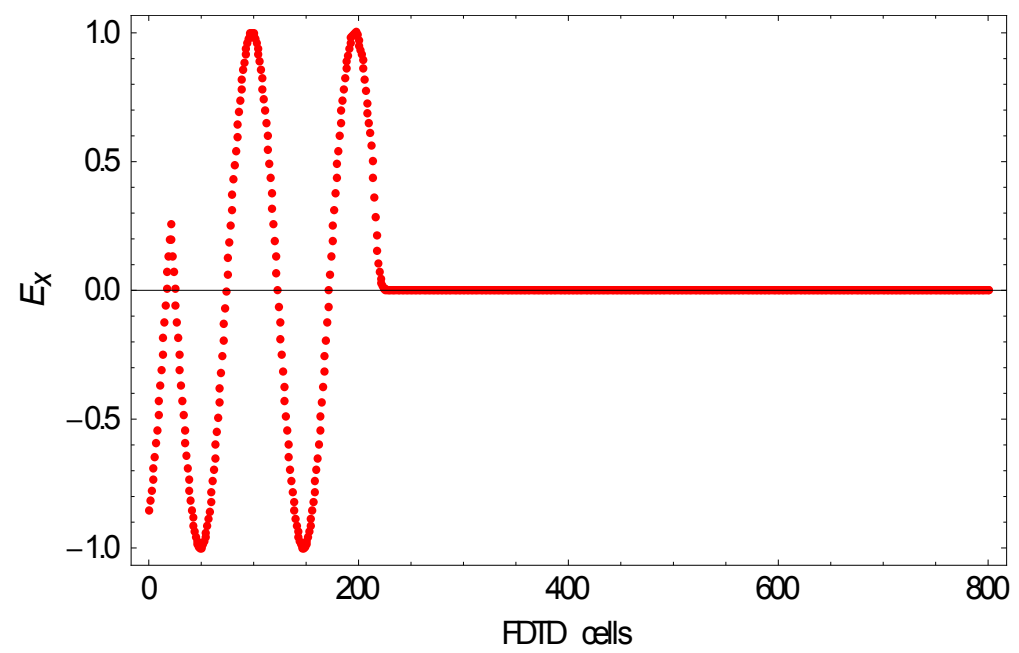

Fig. 6.3. Electric field distribution in free space $(N=400)$. 


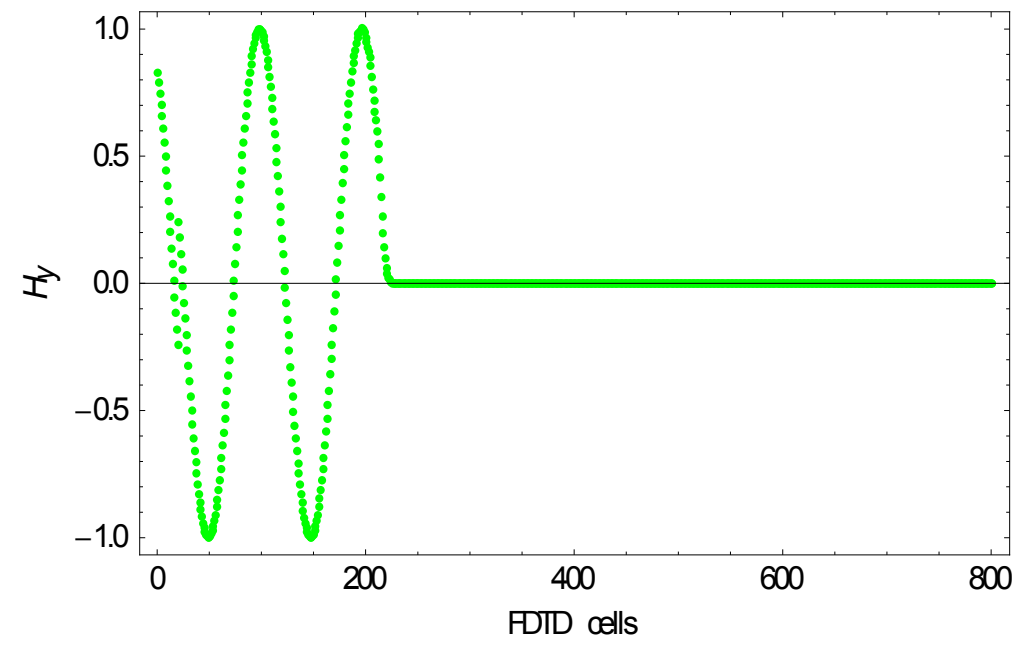

Fig. 6.4. Magnetic field distribution in free space $(N=400)$.

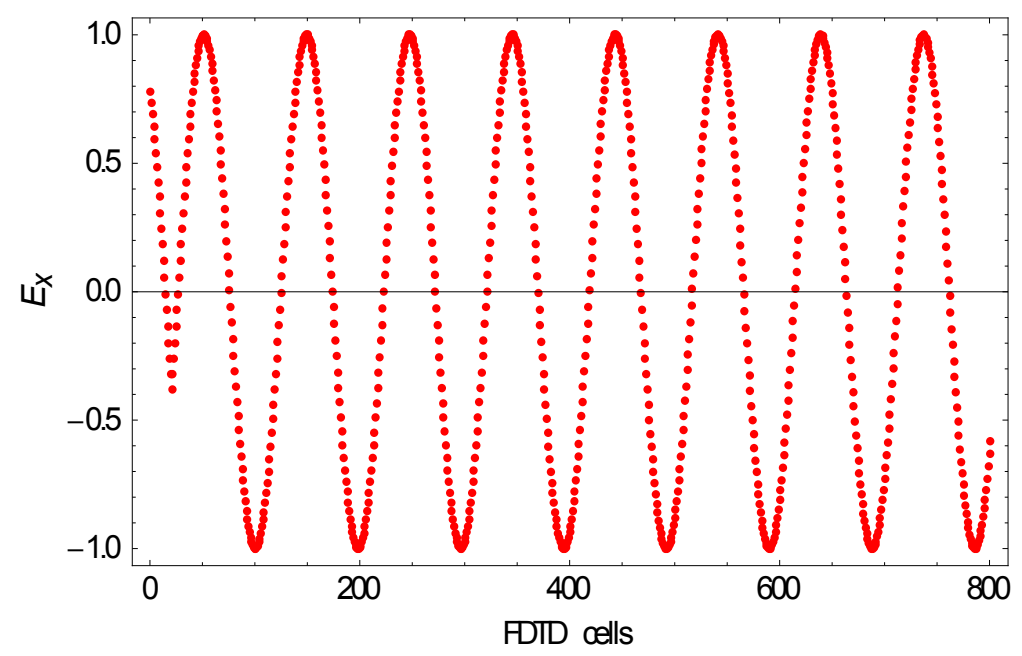

Fig. 6.5. Electric field distribution in free space $(N=5400)$. 


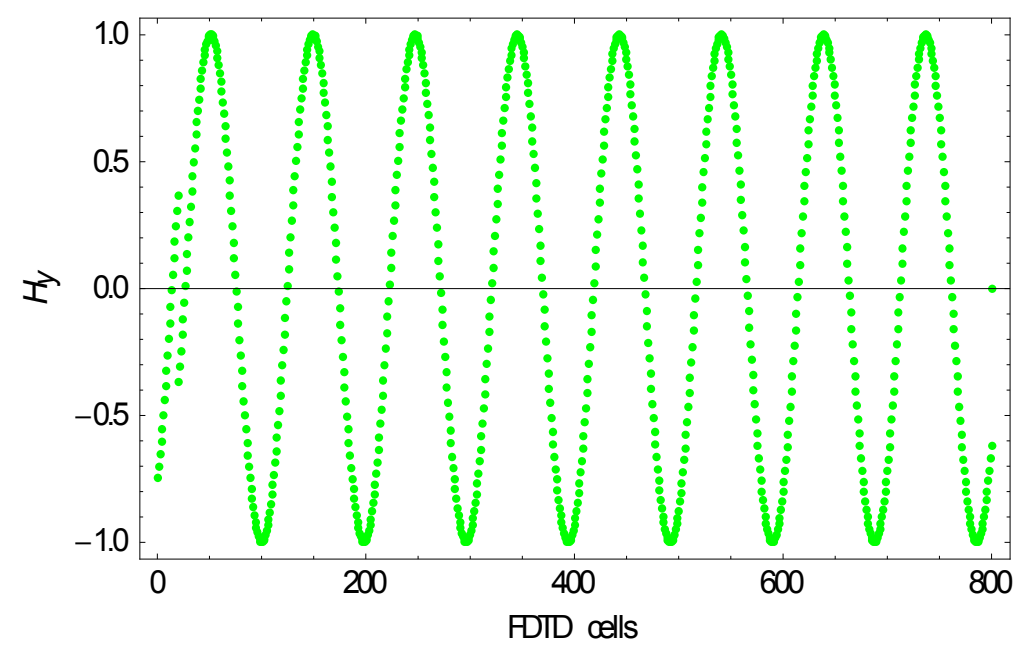

Fig. 6.6. Magnetic field distribution in free space $(N=5400)$.

\subsubsection{Metal}

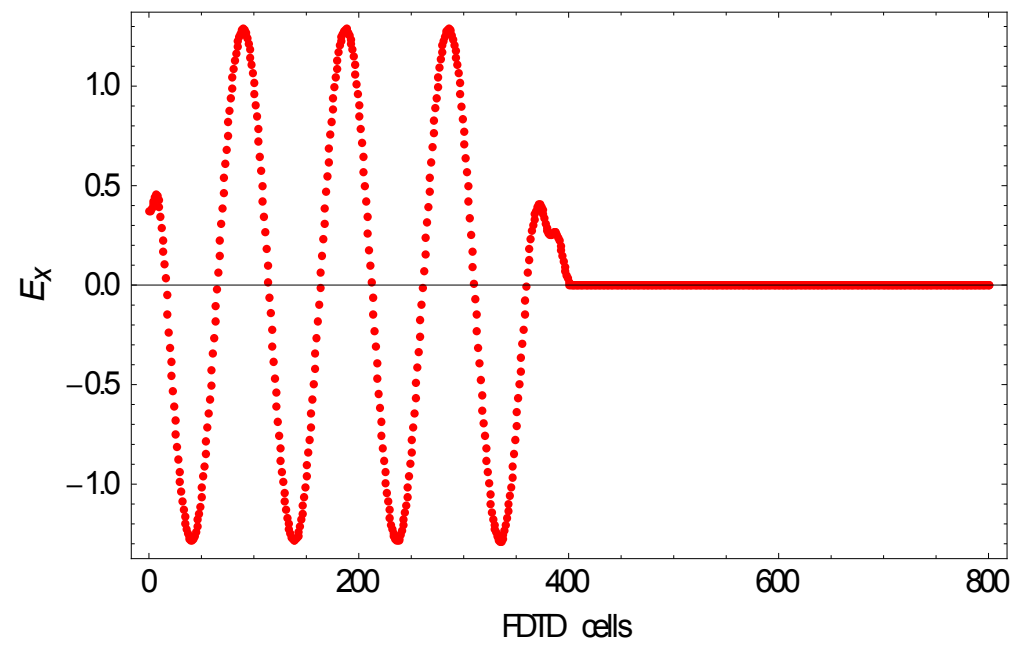

Fig. 6.7. Electric field distribution in metal $(N=5400)$. 


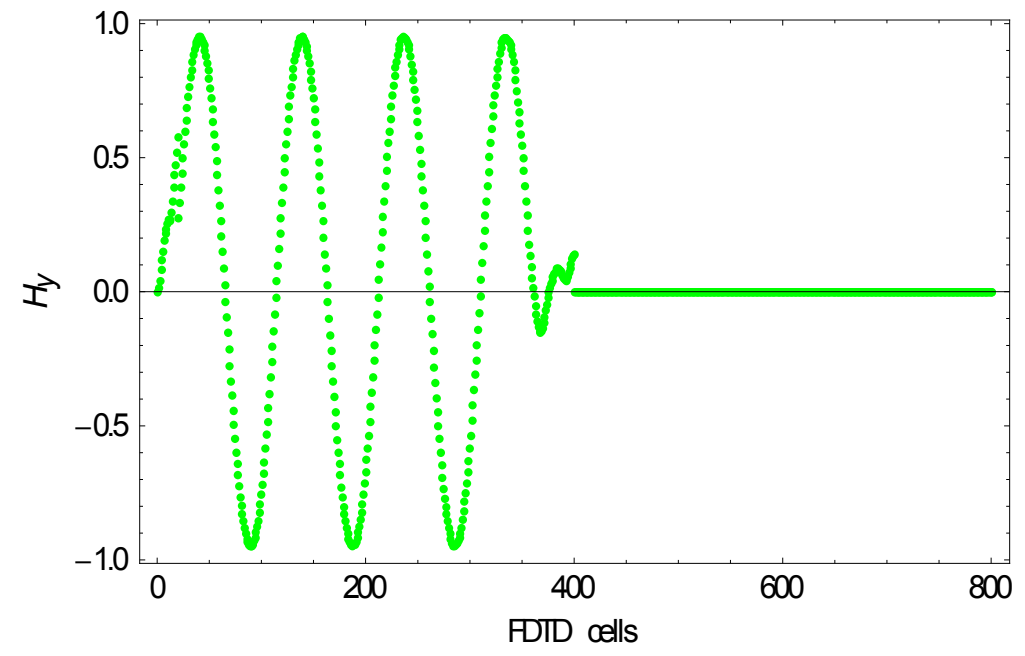

Fig. 6.8. Magnetic field distribution in metal $(N=5400)$.

\subsubsection{Non-lossy Dielectric Medium}

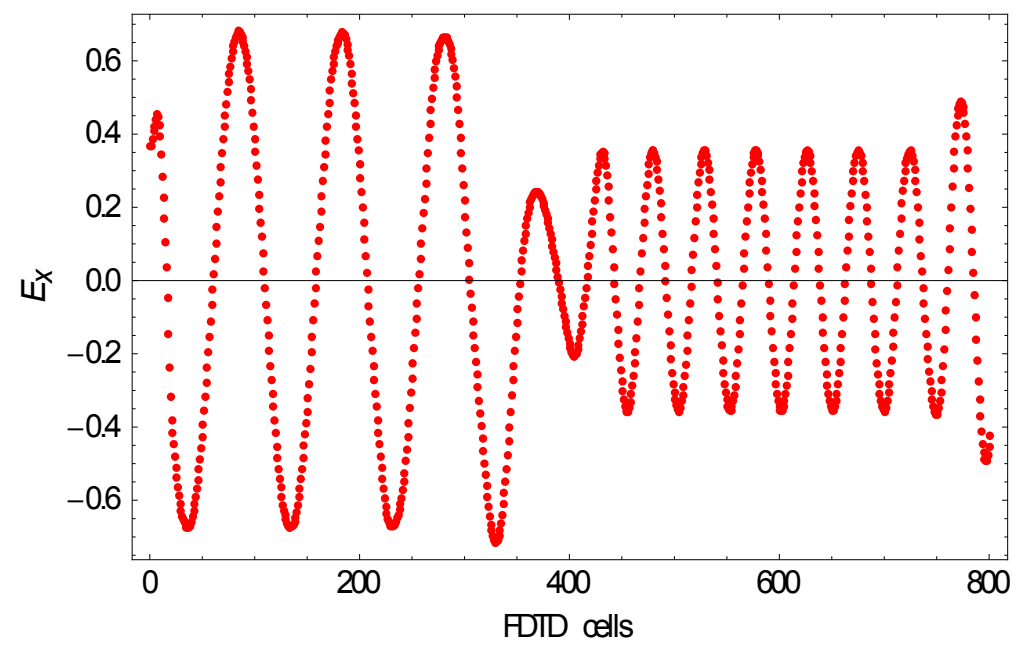

Fig. 6.9. Electric field distribution in the non-lossy dielectric medium $(\mathrm{N}=5400)$. 


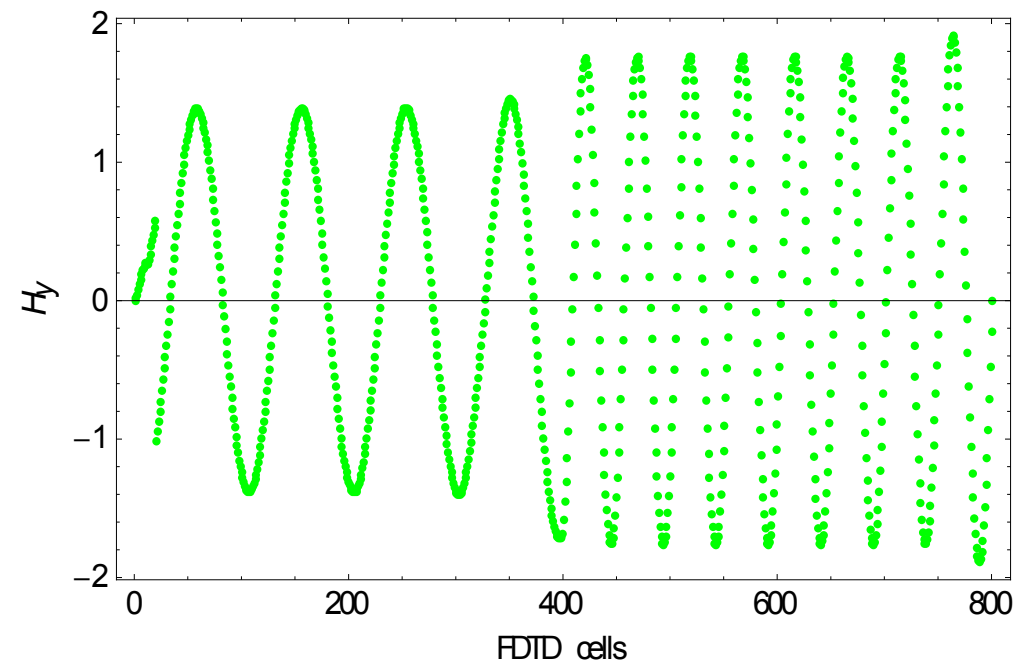

Fig. 6.10. Magnetic field distribution in the non-lossy dielectric medium $(N=5400)$.

\subsubsection{Lossy Dielectric Medium}

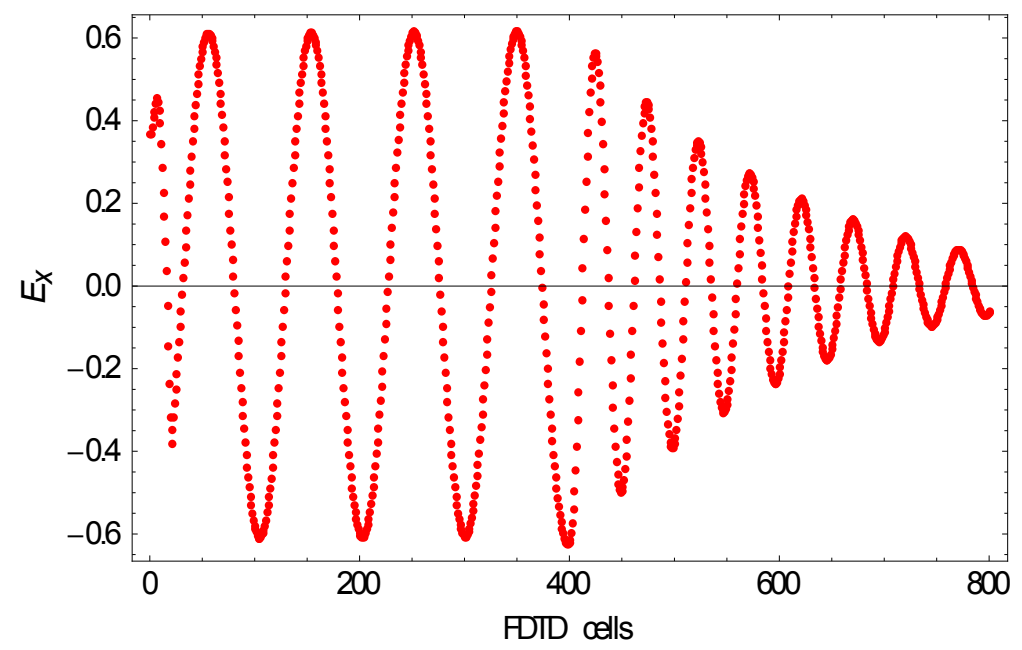

Fig. 6.11. Electric field distribution in the lossy dielectric medium $(N=5400)$. 


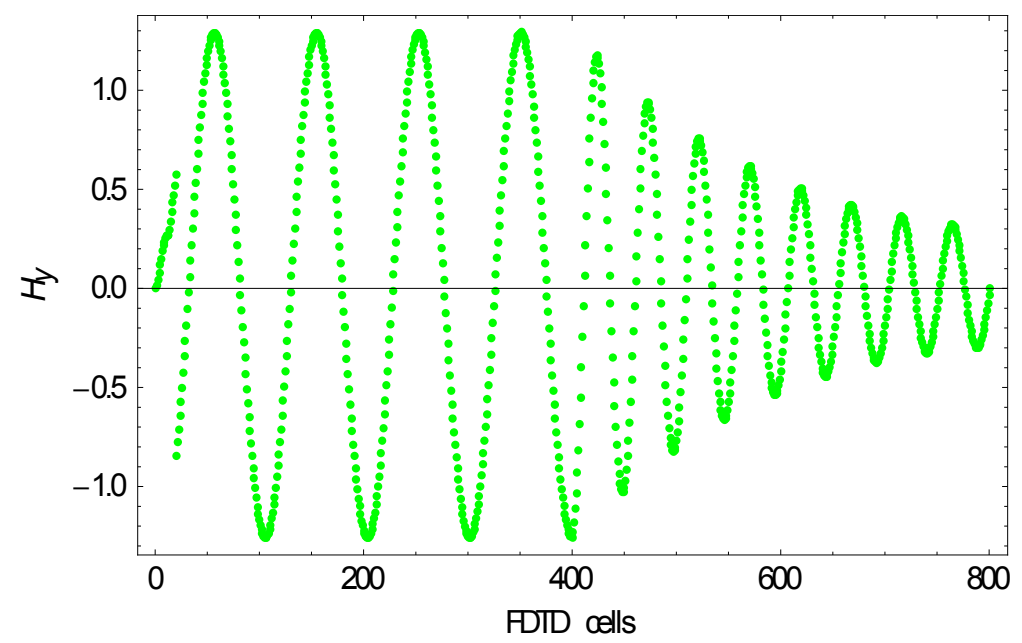

Fig. 6.12. Magnetic field distribution in the lossy dielectric medium $(\mathrm{N}=5400)$.

\subsubsection{Lossy Magnetic Dielectric without Magnetic loss}

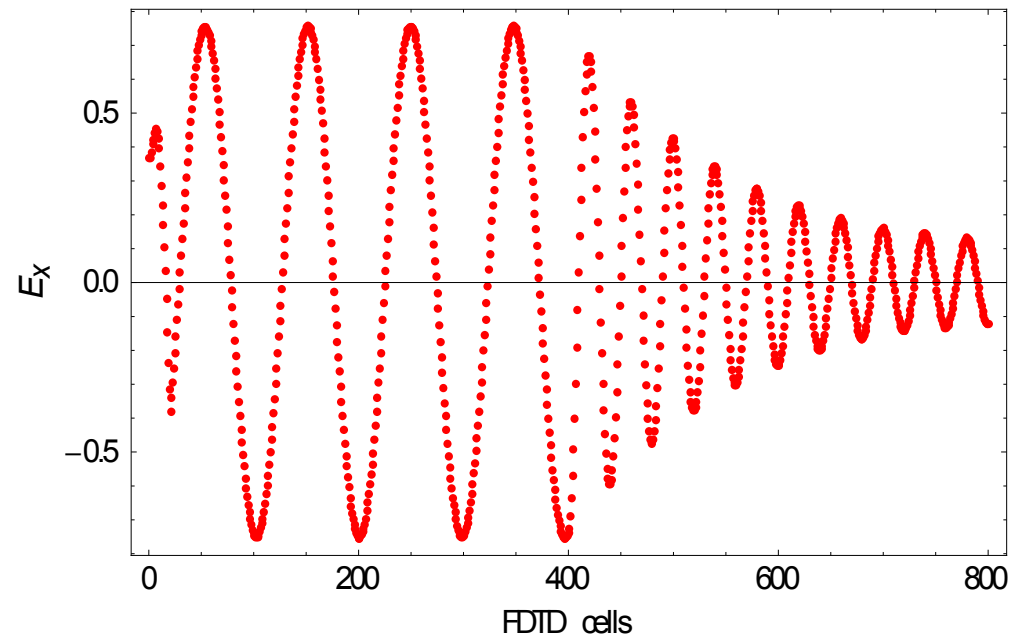

Fig. 6.13. Electric field distribution in the lossy magnetic dielectric without magnetic loss $(\mathrm{N}=\mathbf{5 4 0 0})$. 


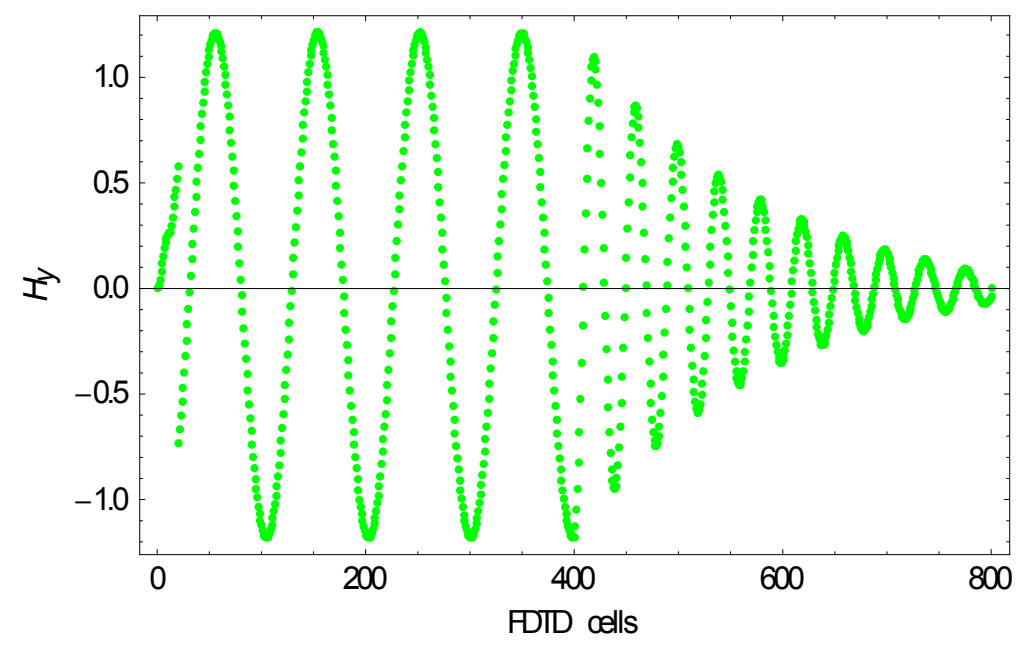

Fig. 6.14. Magnetic field distribution in the lossy magnetic dielectric without magnetic loss $(\mathrm{N}=\mathbf{5 4 0 0})$.

\subsubsection{Lossy Magnetic Dielectric with Magnetic loss}

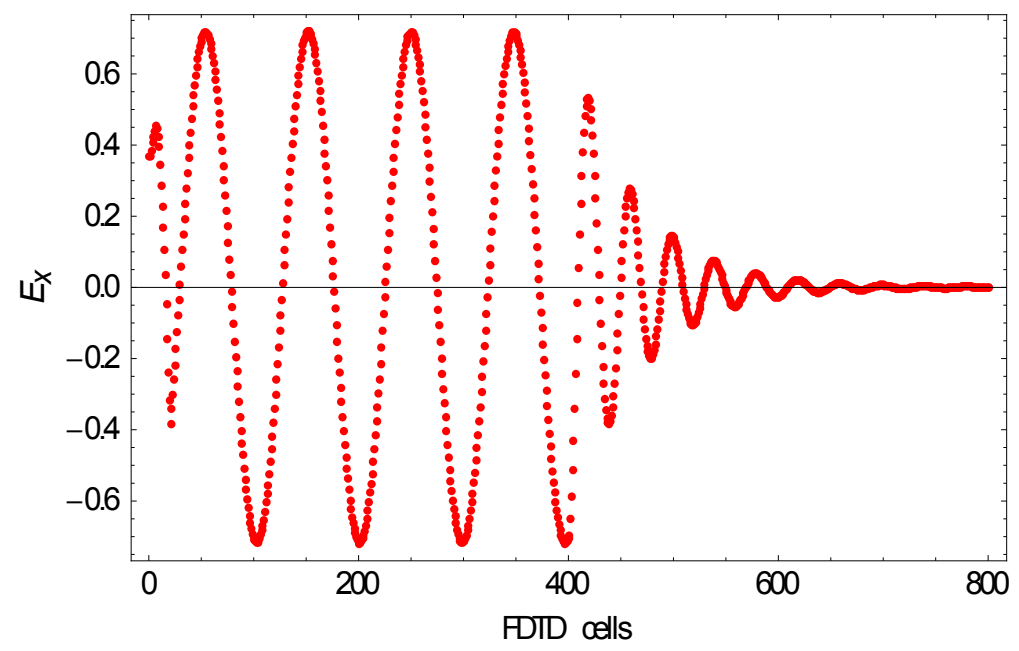

Fig. 6.15. Electric field distribution in the lossy magnetic dielectric with magnetic loss $(\mathrm{N}=$ $5400)$. 


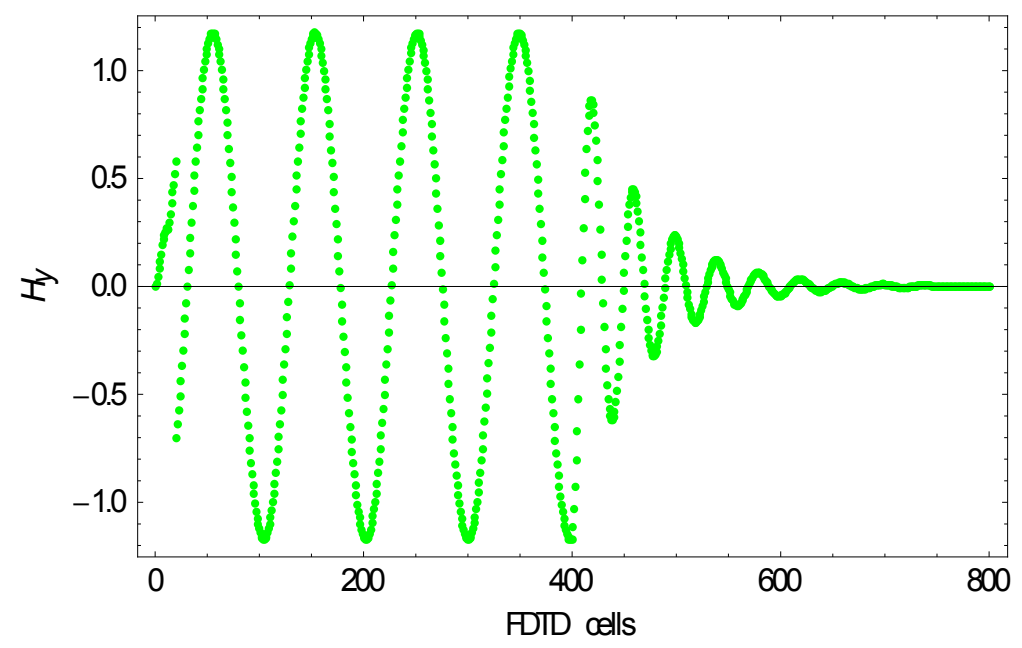

Fig. 6.16. Magnetic field distribution in the lossy magnetic dielectric with magnetic loss (N $=5400)$.

Careful inspection of the above microwave field distribution patterns (e.g., Figs. 6.13 and 6.15) also shows that not only the imaginary parts of permittivity and permeability but also the real parts of permittivity and permeability contribute to microwave decay in the magnetic dielectric materials. This observation is in agreement with the fact that microwave penetration depth depends on both the real part and imaginary part of permittivity and permeability, as indicated in Chapter 3. However, it should be noted that the traditional viewpoint on heat generation (power absorption, as discussed in Chapter 4) in microwave heating does not take into account the effect of real parts of permittivity and permeability. This difference may offer information to account for the non-thermal microwave effects in microwave chemistry.

In fact, non-thermal effect in microwave heating has received considerable attention in the past thirty years and it is still a subject of intense debate in the scientific 108 
community. ${ }^{189-198}$ This effect was assumed to result from a direct stabilizing interaction of the microwave electric field with specific molecules in the dielectric medium which does not associate with the macroscopic temperature effect. ${ }^{195}$ Although a large body of published work has claimed the existence of microwave non-thermal effect, exact reasons for its occurrence is still unknown. Most of the published cases on this topic, which "confirmed" the non-thermal effect, were misinterpreted due to inaccurate temperature measurements by using thermocouple or IR temperature sensors. It thus seems quite difficult to give a definitive answer about the existence or nonexistence of the effect by experiments. More theoretical investigations on the microwave field distributions in various substances may provide reliable evidences for clarifying this issue. 


\title{
Chapter 7 Simulation of Heat Transfer in Microwave
}

\author{
Heating***
}

\subsection{One-dimensional Simulation}

A one-dimensional (1-D) slab of a homogeneous solid having dimension of $2 L$ (Fig. 7.1) heated with microwaves was considered. Microwave energy was assumed to be of uniform intensity and parallel polarization, impinging on both faces of the object. It was delivered in a transverse electric and magnetic (TEM) mode at 915/2450 $\mathrm{MHz}$ and the microwave dissipation in the object followed Lambert's law (a satisfactory approximate alternative to Maxwell's equations applied in microwave heating provided no obvious standing wave pattern forms in materials). ${ }^{124,133}$ Since the same energy was delivered into both sides of the object, giving rise to a temperature distribution with mirror symmetry; thus only one-half of the slab needs to be considered.

\footnotetext{
** The content of this chapter was previously published in ISIJ International ${ }^{199}$ by Zhiwei Peng, JiannYang Hwang, Matthew Andriese, Wayne Bell, Xiaodi Huang, Xinli Wang and in Metallurgical and Materials Transactions $A^{200}$ by Zhiwei Peng, Jiann-Yang Hwang, Chong-Lyuck Park, Byoung-Gon Kim, Gerald Onyedika.

Reproduced with permission from ISIJ International: Zhiwei Peng, Jiann-Yang Hwang, Matthew Andriese, Wayne Bell, Xiaodi Huang, Xinli Wang. Numerical Simulation of Heat Transfer during Microwave Heating of Magnetite. ISIJ International. 2011;51(6):884-888. Copyright (C) 2011 The Iron and Steel Institute of Japan. (See Appendix E-1 for copyright permission).

Reproduced with permission from Metallurgical and Materials Transactions A: Zhiwei Peng, Jiann-Yang Hwang, Chong-Lyuck Park, Byoung-Gon Kim, Gerald Onyedika. Numerical Analysis of Heat Transfer Characteristics in Microwave Heating of Magnetic Dielectrics. Metallurgical and Materials Transactions A. 2012;43A(3):1070-1078. Copyright (C) 2012 The Minerals, Metals and Materials Society. (See Appendix E-2 for copyright permission).
} 


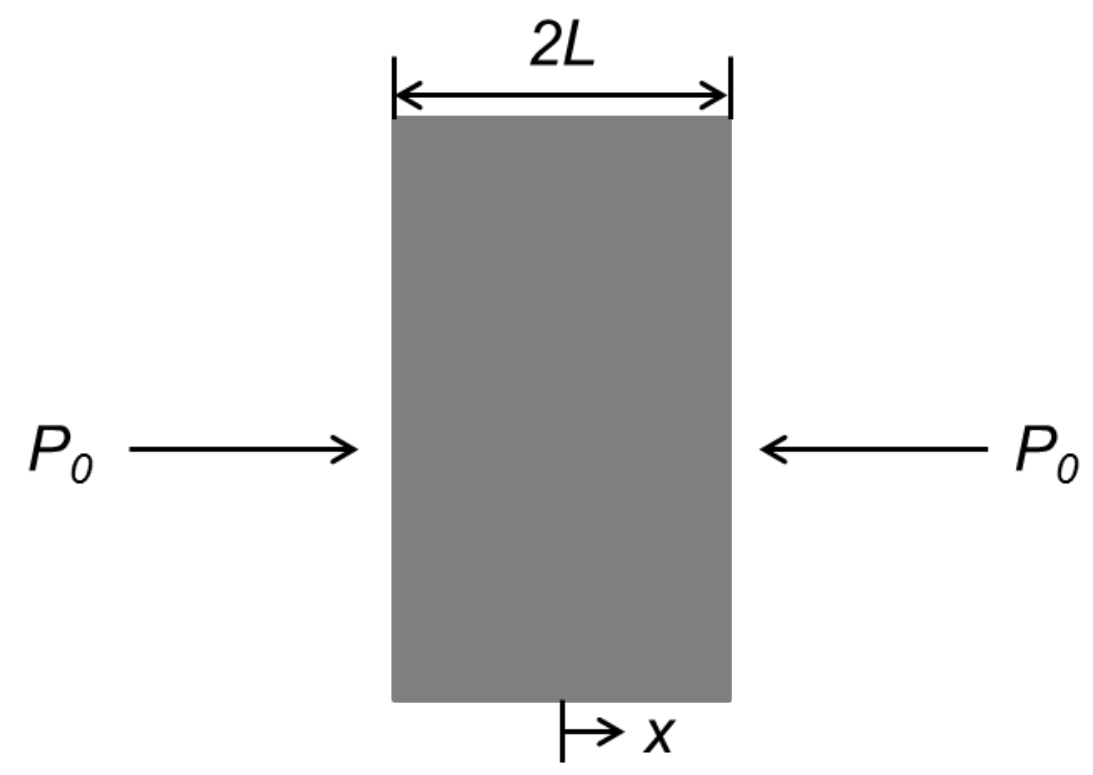

Fig. 7.1. Depiction of the slab geometry.

The mathematical analysis pertinent to the microwave heating process was based on Fourier's law of heat conduction. The shrinkage or deformation of the object during the heating was assumed to be negligible and the surrounding air temperature was considered constant.

The mathematical heat transfer equation governing the microwave heating process in 1$\mathrm{D}(x$ direction $)$ slab was given as ${ }^{109}$

$$
\frac{\partial T}{\partial t}=\frac{1}{\rho c_{p}} \frac{\partial \kappa}{\partial x} \frac{\partial T}{\partial x}+\frac{\kappa}{\rho c_{p}} \frac{\partial^{2} T}{\partial x^{2}}+\frac{P(x)}{\rho c_{p}},
$$

where $T, \rho, c_{p}$, and $\kappa$ are the temperature, density, specific heat capacity, and thermal conductivity, respectively; $P(x)$ is the heat generation term by microwave absorption. 
According to Lambert's law, $P(x)$ can be expressed in terms of microwave power flux $\left(P_{0}\right)$ and penetration depth $\left(D_{p}\right)$ as follows:

$$
P(x)=\frac{P_{0}}{D_{p}} e^{-(L-x) / D_{p}} .
$$

The following initial and boundary conditions were proposed:

$$
\begin{gathered}
t=0, T=T_{0}, 0 \leq \mathrm{x} \leq \mathrm{L}, \\
x=0,-\kappa \frac{\partial T}{\partial x}=0, t>0, \\
x=L,-\kappa \frac{\partial T}{\partial x}=h\left(T-T_{\infty}\right)+\varepsilon \sigma\left[(T+273.15)^{4}-\left(T_{\infty}+273.15\right)^{4}\right], t>0,
\end{gathered}
$$

where $t, T_{0}, h, T_{\infty}, \varepsilon$, and $\sigma$ denote, respectively, time, initial temperature, heat transfer coefficient, environmental temperature, emissivity, and the Stefan-Boltzmann constant.

The method used in this study was the explicit finite-difference approximation, where the governing equations were transformed into difference equations by dividing the domain of solution to a grid of points in the form of mesh and the derivatives were expressed along each mesh point, referred as a node. The spatial domain $[0, L]$ was divided into $m$ sections, each of length $\Delta x=L / m$. Meanwhile, the time domain $[0, t]$ was divided into $n$ segments, each of duration $\Delta t=t / n$. The index $i$ represents the mesh points in the $x$ direction, starting with $i=0$ being one boundary (slab center) and ending at $i=m$ (slab surface). Specifically, the following difference equations were used:

$$
\frac{\partial T}{\partial t}=\frac{T_{i}^{n+1}-T_{i}^{n}}{\Delta t},
$$




$$
\begin{gathered}
\frac{\partial T}{\partial x}=\frac{T_{i+1}^{n}-T_{i-1}^{n}}{2 \Delta x}, \\
\frac{\partial^{2} T}{\partial x^{2}}=\frac{T_{i+1}^{n}+T_{i-1}^{n}-2 T_{i}^{n}}{(\Delta x)^{2}} .
\end{gathered}
$$

To evaluate the thermal conductivity spatial derivative in eq. (7.1), the following equation was applied:

$$
\frac{\partial \kappa}{\partial x}=\frac{\kappa_{i+1}^{n}-\kappa_{i-1}^{n}}{2 \Delta x}
$$

By substituting above difference equations into the heat transfer equation and the equations of initial and boundary conditions, the temperature of the sample at a given time could be determined. The solution was found by developing a computer code in a Mathematica 7.0 program.

The material considered in the simulation is magnetite derived from magnetite concentrate in Tilden Mine, Michigan. The thermophysical properties of the material and modeling parameters are tabulated in Table 7.1..$^{65,201-203}$ 
Table 7.1

Thermophysical properties and modeling parameters used in the simulation.

\begin{tabular}{lll}
\hline Parameter & Value & Unit \\
\hline$\kappa$ & $3.8558-1.37 \times 10^{-3} T^{*}$ & $\mathrm{~W} \mathrm{~K}^{-1} \mathrm{~m}^{-1}$ \\
$c_{p}$ & $611.84+1.384 T^{\S}$ & $\mathrm{J} \mathrm{kg}^{-1}{ }^{\circ} \mathrm{C}^{-1}$ \\
$\rho^{*}$ & $2800^{\ddagger}$ & $\mathrm{kg} \mathrm{m}^{-3}$ \\
$\alpha^{*}\left(9.001377-4.8929 \times 10^{-7} T\right) /(611.84+1.384 T)$ & $\mathrm{m}^{2} \mathrm{~s}^{-1}$ \\
$D_{p}(915 \mathrm{MHz})$ & $0.0471-0.453 \times 10^{-5} T+4.184 \times 10^{-7} T^{2}-4.845 \times 10^{-9} T^{3}+1.298 \times 10^{-11} T^{4}-$ & $\mathrm{m}$ \\
& $1.366 \times 10^{-14} T^{5}+5.099 \times 10^{-18} T^{6 \ddagger}$ & \\
$D_{p}(2450 \mathrm{MHz})$ & $0.0161-1.863 \times 10^{-5} T+2.182 \times 10^{-7} T^{2}-1.603 \times 10^{-9} T^{3}+0.395 \times 10^{-11} T^{4}-$ & $\mathrm{m}$ \\
& $0.405 \times 10^{-14} T^{5}+1.502 \times 10^{-18} T^{6 \ddagger}$ & \\
$h$ & 10 & $\mathrm{~W} \mathrm{~m}{ }^{-2}{ }^{\circ} \mathrm{C}^{-1}$ \\
$\varepsilon$ & $0.96^{\dagger}$ & $\mathrm{None}$ \\
$T_{0}$ & 25 & ${ }^{\circ} \mathrm{C}$ \\
$T_{\infty}$ & 25 & ${ }^{\circ} \mathrm{C}$ \\
\hline
\end{tabular}

* Value calculated based on the data reported in ref. 201.

$\S$ Value calculated based on the data reported in ref. 202.

${ }^{\ddagger}$ Values taken from ref. 65.

${ }^{\dagger}$ Value taken from ref. 203.

\subsubsection{Effect of Heating Time}

The temperature profiles for different heating time periods ranging from 1 to $60 \mathrm{~s}$ (at $915 \mathrm{MHz}$ ) are shown in Fig. 7.2. The highest temperatures inside the object are around $36{ }^{\circ} \mathrm{C}, 122{ }^{\circ} \mathrm{C}, 294{ }^{\circ} \mathrm{C}$, and $767{ }^{\circ} \mathrm{C}$ for $1 \mathrm{~s}, 10 \mathrm{~s}, 30 \mathrm{~s}$, and $60 \mathrm{~s}$, respectively. Temperature in the object increases rapidly with time due to the increase in thermal energy transformed from the microwave irradiation. Continued microwave heating creates a nonuniform temperature distribution in the slab. The temperature of slab center $(L=0 \mathrm{~m})$ stays colder $\left(37^{\circ} \mathrm{C}\right)$ after heating for $60 \mathrm{~s}$, giving an indication that the thermal runaway may occur during the microwave heating. Additionally, the surface of the object $(L=0.2 \mathrm{~m}, L / \Delta x=400)$ is found to be the position with the highest temperature in the initial period $(\sim 1 \mathrm{~s})$. Longer heating $(>60 \mathrm{~s})$ leads to a temperature 
peak, which migrates inward with time, as represented in Fig. 7.3. It is mainly attributed to the effects of microwave heat generation and thermal radiation. In the initial heating, the thermal contribution from microwave generation dominates the temperature rise in the sample and weak thermal radiation effect could be expected due to relatively low temperature of the object. As the heating continues the temperature of object increases considerably, leading to a high radiation effect. Thus, an obvious temperature peak is formed inside the object after relatively long heating time. Note that heat diffusion and convection also contribute to the heat transfer in microwave heating of materials. But for the magnetite in this study (actually, for many ceramic materials), their contributions are quite small, especially the heat diffusion. The heat diffusivity $\left(\alpha^{*}\right.$ in Table 7.1 and Fig. 7.4) is found to be in the order of $10^{-6} \mathrm{~m}^{2} \mathrm{~s}^{-1}$ and decreases with increasing temperature.

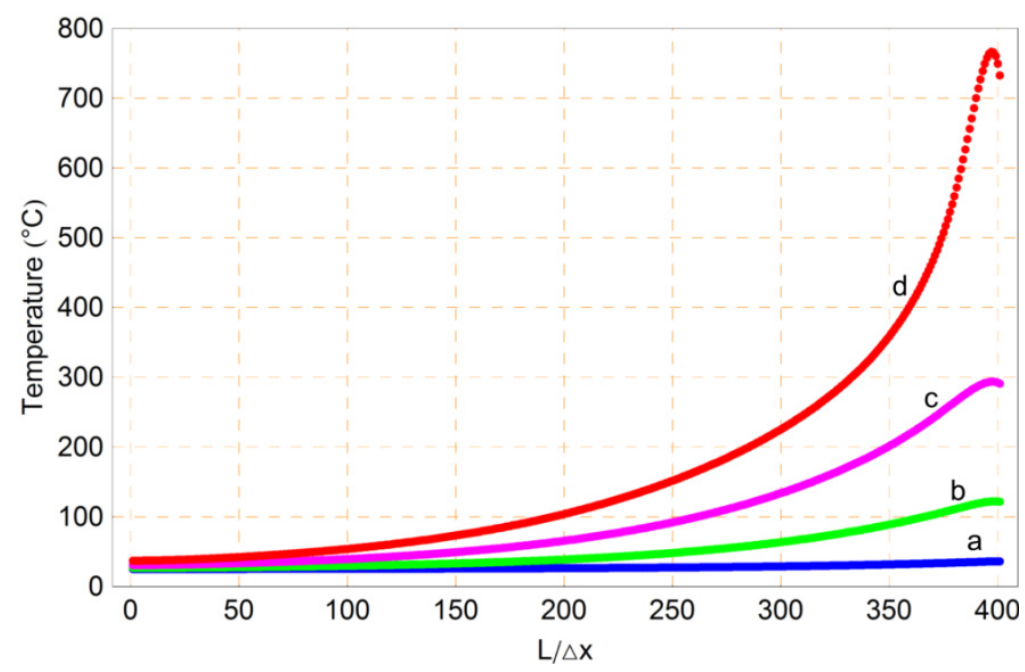

Fig. 7.2. Temperature distributions in the magnetite slab for different microwave heating periods at 915 MHz: a-1 s, b-10 s, c-30 s, and d-60 s. Power: $1 \mathrm{MW} \mathrm{m}^{-2}$; Dimension $(L): 0.2 \mathrm{~m}$. 


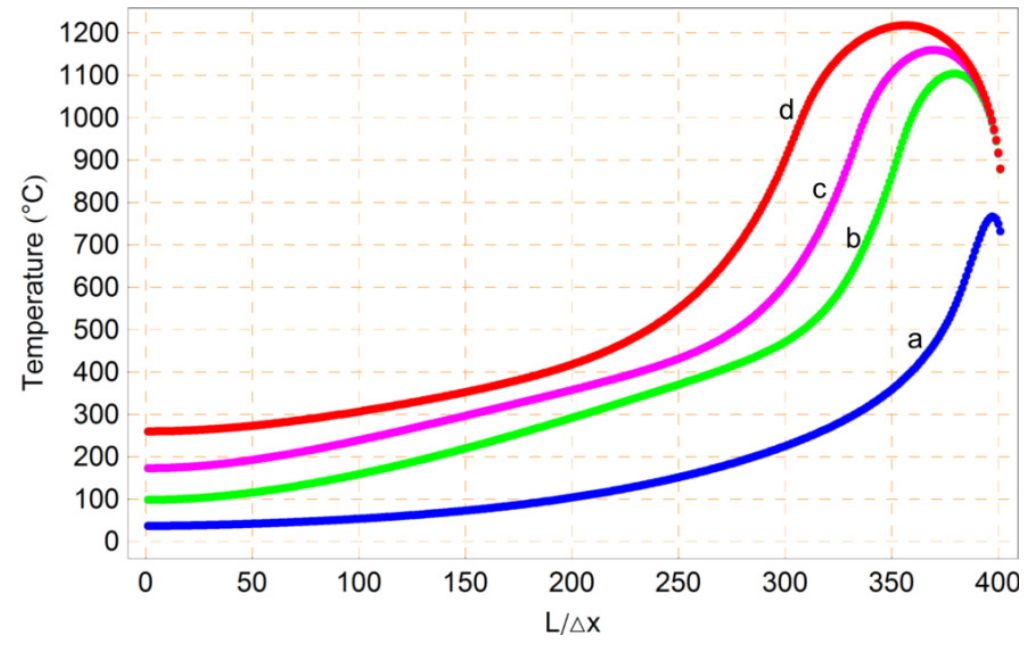

Fig. 7.3. Temperature distributions in the magnetite slab for different microwave heating

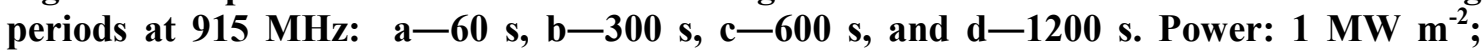
Frequency: 915 MHz; Dimension (L): $0.2 \mathrm{~m}$.

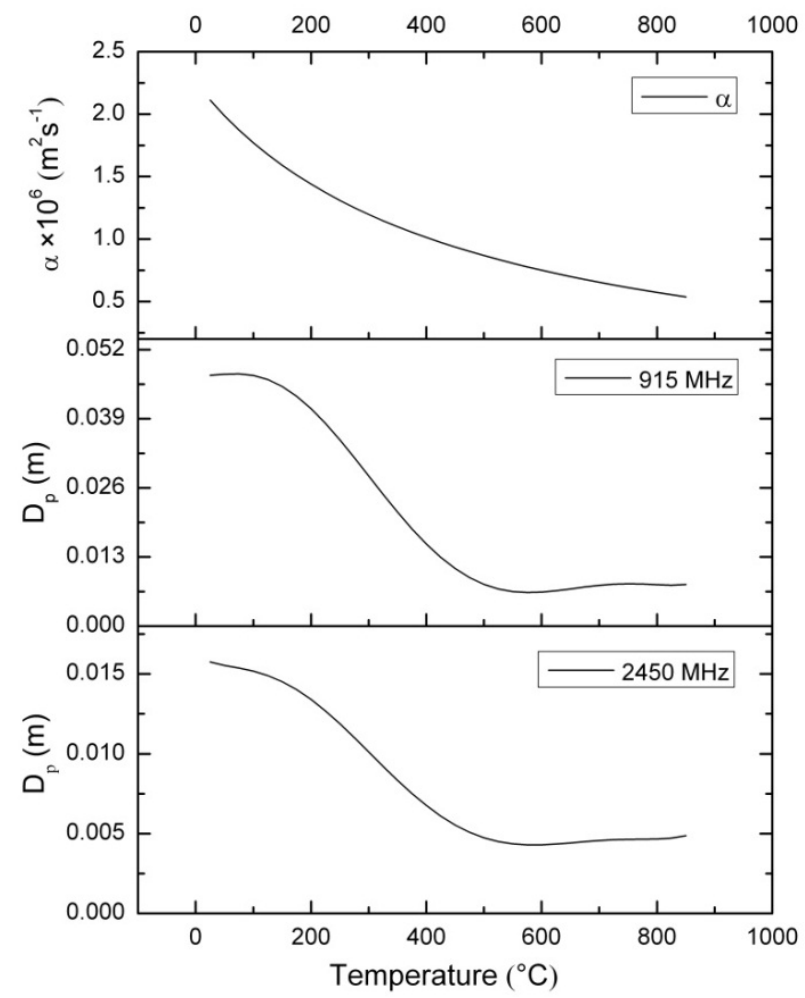

Fig. 7.4. Temperature dependences of magnetite thermal diffusivity $(\alpha)$ and microwave penetration depth $\left(D_{p}\right)$. 


\subsubsection{Effect of Heating Power}

The temperature profiles for different microwave powers $\left(P_{0}\right)$ in the range of 0.5 to 4 MW $\mathrm{m}^{-2}$ are given in Fig. 7.5. The temperature of the object increases with increasing microwave power. The highest temperatures after microwave heating for $60 \mathrm{~s}$ are 278 ${ }^{\circ} \mathrm{C}, 767{ }^{\circ} \mathrm{C}, 1047{ }^{\circ} \mathrm{C}$, and $1143{ }^{\circ} \mathrm{C}$ for $0.5 \mathrm{MW} \mathrm{m}^{-2}, 1 \mathrm{MW} \mathrm{m}^{-2}, 2 \mathrm{MW} \mathrm{m}^{-2}$, and $4 \mathrm{MW}$ $\mathrm{m}^{-2}$, respectively. It demonstrates that a suitable power applied in microwave heating is crucial to obtain high heating rate in a short time. Moreover, it is interesting to note that these highest temperatures locate at different mesh positions $(L / \Delta x): 396,396,390$, and 385 , respectively. It shows the temperature peak shifts to the center of the object with increasing power as the contribution of microwave heat generation from higher power to temperature increase becomes even considerable compared with heat convection and diffusion. Owing to the slow heat diffusion and strong heat radiation to environment at high temperatures the peak migrates inward to keep heat balance between the object and surrounding. 


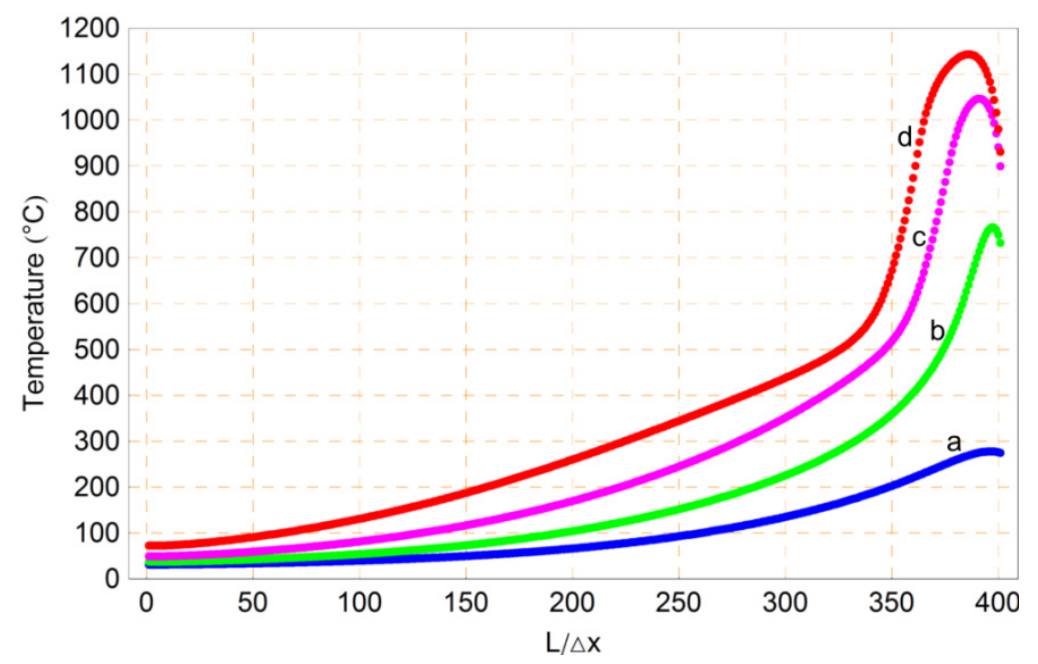

Fig. 7.5. Temperature distributions in the magnetite slab under different microwave

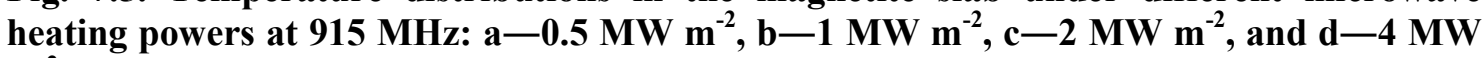
$\mathrm{m}^{-2}$. Heating time: $60 \mathrm{~s}$; Dimension $(L): 0.2 \mathrm{~m}$.

\subsubsection{Effect of Microwave Frequency}

In microwave processing of materials, the dissipation of microwave power in materials highly relies on the microwave frequency. It is known that two frequencies, 915 and $2450 \mathrm{MHz}$, are commonly assigned for industrial and domestic applications. To evaluate the effect of microwave frequency on temperature distribution in magnetite, the temperature profiles in the object for different microwave heating periods at frequency of $2450 \mathrm{MHz}$ are shown in Fig. 7.6 with a comparison with $915 \mathrm{MHz}$ in Fig. 7.2. The comparison indicates there is negligible temperature difference between 915 and $2450 \mathrm{MHz}$ in the initial heating period (1 s). As the heating time extends to $60 \mathrm{~s}$, the maximum temperature of the object at $2450 \mathrm{MHz}$ is found to be much higher than that at $915 \mathrm{MHz}$ (996 and $767{ }^{\circ} \mathrm{C}$, respectively) and the heating rate is consistent with the 
experimental data reported in literature. ${ }^{204}$ The heating rate difference between two frequencies is attributed to the different microwave wavelengths and microwave absorption properties (permittivity and permeability) of the material at 915 and 2450 MHz. Their effects on the heating can be indicated by the change in microwave penetration depth $\left(D_{p}\right)$ in the material [see eq. (3.22)].

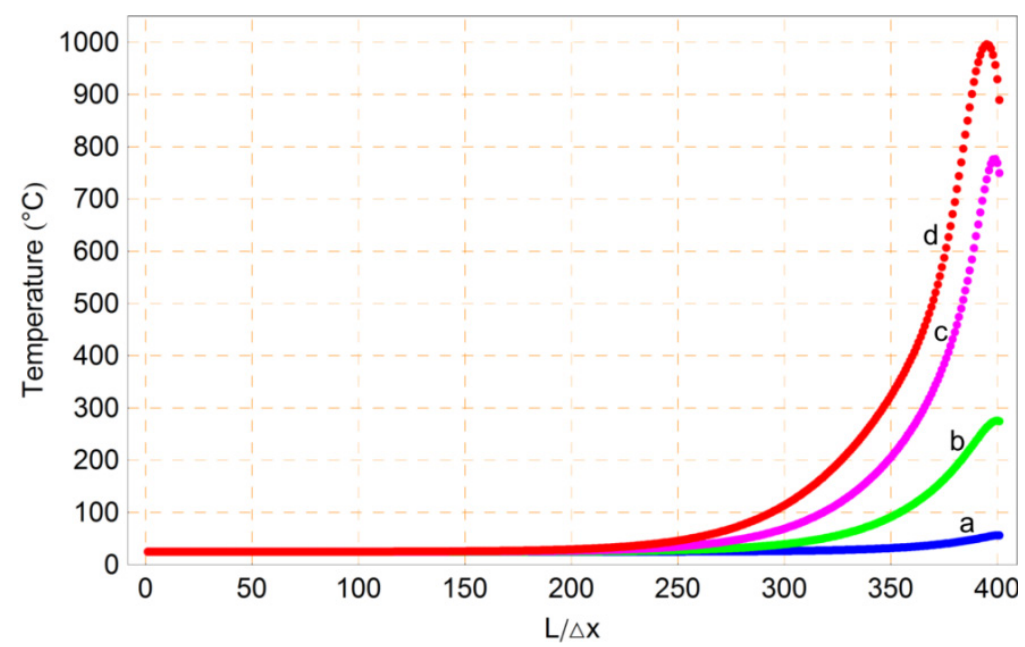

Fig. 7.6. Temperature distributions in the magnetite slab for different microwave heating periods at 2450 MHz: a-1 s, b-10 s, c-30 s, and d-60 s. Power: $1 \mathrm{MW} \mathrm{m}^{-2}$; Dimension (L): $0.2 \mathrm{~m}$.

In this simulation, the temperature dependences of microwave penetration depths at 915 $\mathrm{MHz}$ and $2450 \mathrm{MHz}$ were determined via the cavity perturbation technique, as shown in Table 7.1 and Fig. 7.4. The microwave penetration depth at $2450 \mathrm{MHz}$ is found to be much smaller than that at $915 \mathrm{MHz}$ below $500{ }^{\circ} \mathrm{C}$, mainly due to their different microwave wavelengths. As temperature increases, the microwave penetration depth is also greatly affected by the permittivity and permeability. The magnetite permittivity increases with temperature, while the permeability decreases apparently around the 
Curie point. Note that the magnitude of permittivity is much larger than that of permeability. Thus, the change in permittivity dominates the variation of microwave penetration depth in magnetite at high temperatures.

The small microwave penetration depth at $2450 \mathrm{MHz}$ results in a quick temperature increase in a short time (e.g., $<60 \mathrm{~s})$. This indicates, under the same conditions (power, heating time, object dimension, etc.), most of microwave energy at $2450 \mathrm{MHz}$ would dissipate in the area closer to surface than that at $915 \mathrm{MHz}$. As microwave heating continues, the temperature of object increases and the radiation effect at the surface of object becomes quite strong. The difference of the highest temperatures between 915 and $2450 \mathrm{MHz}$ decreases, as shown in Figs. 7.3 and 7.7. The highest temperatures at $915 \mathrm{MHz}$ after microwave heating for $60 \mathrm{~s}, 300 \mathrm{~s}, 600 \mathrm{~s}$, and $1200 \mathrm{~s}$ are $767{ }^{\circ} \mathrm{C}, 1104$ ${ }^{\circ} \mathrm{C}, 1160{ }^{\circ} \mathrm{C}$ and $1218{ }^{\circ} \mathrm{C}$, respectively. At $2450 \mathrm{MHz}$, the counterparts are $996{ }^{\circ} \mathrm{C}$, $1154{ }^{\circ} \mathrm{C}, 1215^{\circ} \mathrm{C}$, and $1285{ }^{\circ} \mathrm{C}$, respectively. Furthermore, owing to more energy is located in the section close to the surface, the temperature inside the object at 2450 $\mathrm{MHz}$ is much lower than that at $915 \mathrm{MHz}$. In other words, in the investigated heating time range, the temperature distribution at $915 \mathrm{MHz}$ is more uniform than that at 2450 MHz. Hence, $915 \mathrm{MHz}$ is more suitable for large-scale microwave heating of magnetite where the maximum temperature uniformity is demanded. 


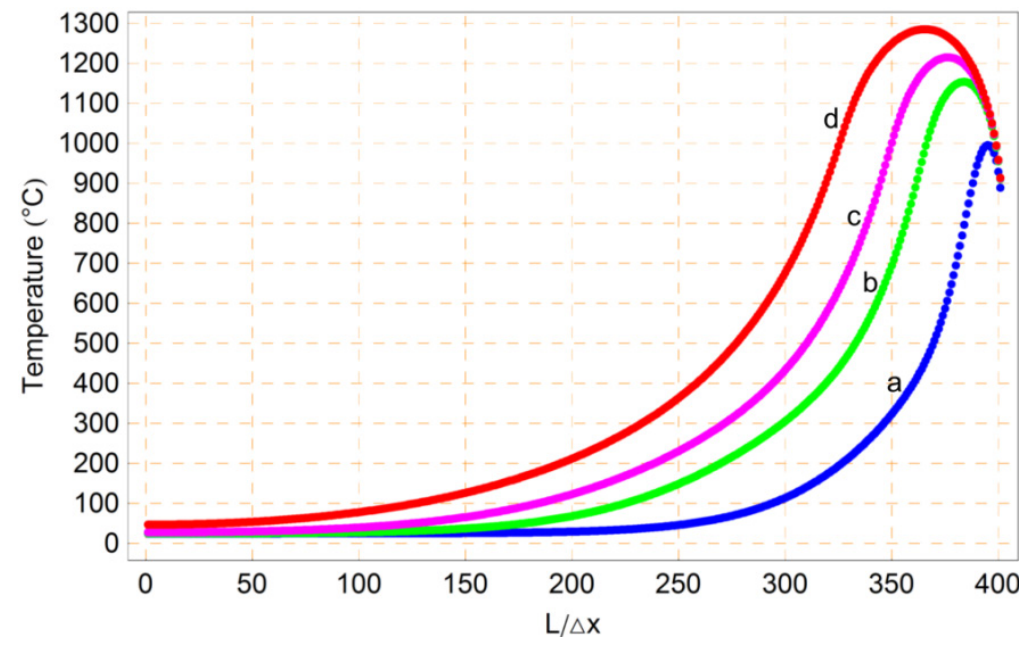

Fig. 7.7. Temperature distributions in the magnetite slab for different microwave heating

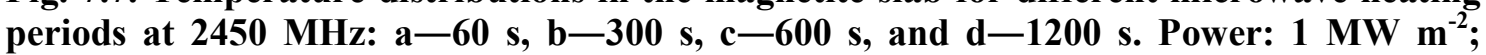
Dimension $(L): 0.2 \mathrm{~m}$.

\subsubsection{Effect of Object Dimension}

Volumetric heating is known as a main advantage of microwave processing of materials due to the propagation behaviors of microwaves. ${ }^{205}$ However, this superiority also depends on the object dimension, as demonstrated in Fig. 7.8. It shows the temperatures for the slab with different dimensions $(L=0.2 \mathrm{~m}, 0.15 \mathrm{~m}, 0.1 \mathrm{~m}$, and $0.05 \mathrm{~m}$, respectively) after microwave heating for $60 \mathrm{~s}$ at $2450 \mathrm{MHz}$. As the dimension decreases, the temperature homogeneity in the object is improved. The temperature peak magnitude remains almost constant while its position moves close to the center of the object. The object with dimension $(L)$ of $0.05 \mathrm{~m}$ under microwave irradiation exhibits a better temperature distribution than the others. This could be clearly demonstrated by the temperature increase at the slab center with decreasing dimension. 
The temperature at the slab center increases from 25 to $153{ }^{\circ} \mathrm{C}$ as the dimension decreases from 0.2 to $0.05 \mathrm{~m}$. This indicates an optimal dimension of the material is required to obtain the minimum temperature nonuniformity and high heating performance. Also, it should be noted that further reduction of dimension size (e.g., $L=$ $0.02 \mathrm{~m}$ ) would result in an apparent standing wave pattern, which may dramatically worsen the heating uniformity. ${ }^{206,207}$

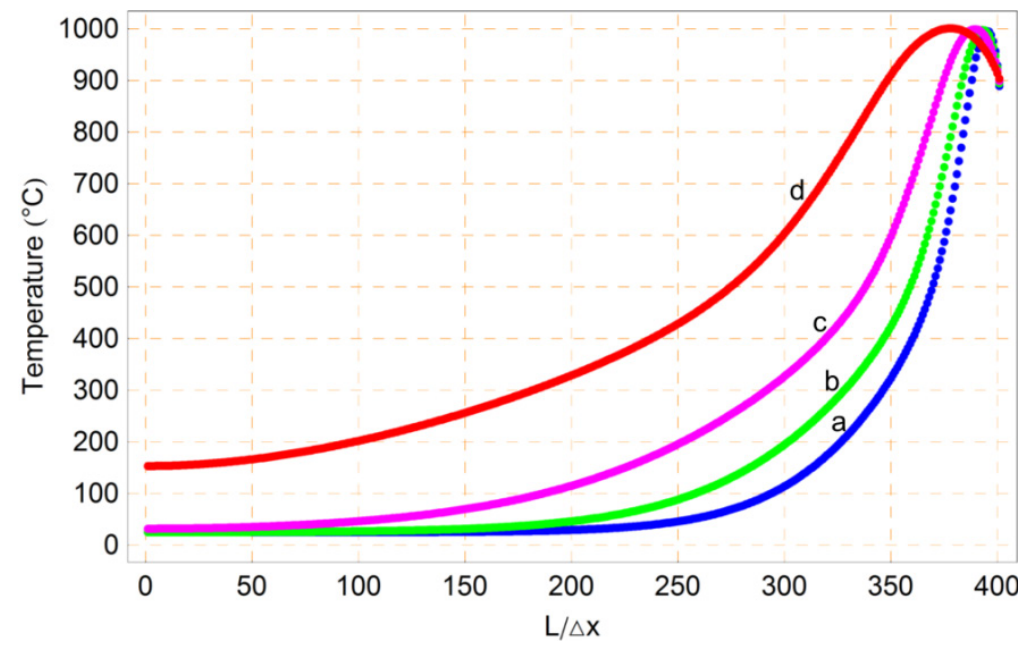

Fig. 7.8. Temperature distributions in the magnetite slab with different dimensions $(L)$ at 2450 MHz: $a-0.2 \mathrm{~m}, \mathrm{~b}-0.15 \mathrm{~m}, \mathrm{c}-0.1 \mathrm{~m}$, and $\mathrm{d}-0.05 \mathrm{~m}$. Heating time: $60 \mathrm{~s}$; Power: 1 MW $\mathbf{m}^{-2}$.

\subsection{Two-dimensional Simulation}

Microwave heating highly depends on the dimension of material. To visualize a clearer heat transfer process a two-dimensional (2-D) simulation was also performed. In the modeling, a 2-D homogeneous magnetite block having dimension of $2 L \times 2 L$ heated 
with microwaves with power, $P_{0}$, was considered. Microwave energy was assumed to be of uniform intensity and parallel polarization, impinging on four faces of the object in a transverse electric and magnetic (TEM) mode at specified frequencies. Owing to the two-fold symmetry of the block, only the temperature distribution of one-quarter of the object (upper right part in this simulation) was simulated, as shown in Fig. 7.9. The temperature distribution inside the 2-D object was predicted through the numerical method discussed above. Magnetite was taken as the object considered in the simulation and its thermophysical properties and the modeling parameters are used as the same in the 1-D case.

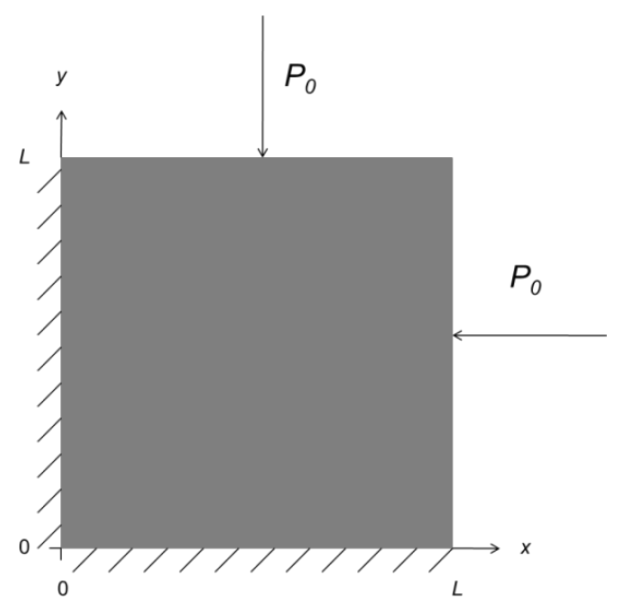

Fig. 7.9. Depiction of the 2-D object (one-quarter) geometry.

\subsubsection{Effect of Heating Time}

The temperature distributions in the object for different heating time periods ranging from 1 to $600 \mathrm{~s}$ (at $915 \mathrm{MHz}$ ) are given in Fig. 7.10. The highest temperatures 
(temperature of hot spot) inside the object are around $45^{\circ} \mathrm{C}, 1000{ }^{\circ} \mathrm{C}, 1200{ }^{\circ} \mathrm{C}$, and $1200{ }^{\circ} \mathrm{C}$ for $1 \mathrm{~s}, 60 \mathrm{~s}, 300 \mathrm{~s}$, and $600 \mathrm{~s}$, respectively. Temperature in the object increases rapidly in one minute as the time period progresses. This is because of the increase in thermal energy transformed from the microwave radiation due to high dielectric and magnetic loss of magnetite with increasing heating time. Also, it is apparent that the temperature distribution inside the object is nonuniform, giving an indication that the thermal runaway may occur during the experiment. In the initial period $(\sim 1 \mathrm{~s})$, the upper-right corner of the object $(L=0.2 \mathrm{~m})$ is found to be the position with the highest temperature. As the heating time increases, the maximum temperature position shifts towards the center of the object and the temperature of the object surface is tending to be lower than the inside section. This is mainly attributed to the heat balance between the microwave heat generation and thermal radiation. In the initial heating period, the thermal contribution from microwave generation dominates the temperature rise in the sample and the thermal radiation effect is weak due to a relatively low temperature of the object. As the heating time extends, the temperature of the object increases considerably, giving rise to a high radiation effect. Thus, as shown in Fig. 7.10, an obvious hot spot is formed inside the object after a relatively long heating time (300 s). Note that continuous heating up to $600 \mathrm{~s}$ did not contribute too much to the increase in the highest temperature inside and the temperature of the hot spot remains around 1200 ${ }^{\circ} \mathrm{C}$. It is also observed that the hot spot migrates inward with a slower speed due to smaller heat diffusivity $\left(\alpha^{*}\right.$ in Table 7.1$)$ at higher temperatures. This indicates that the microwave heat generation overwhelms the heat diffusion during the microwave 
heating of magnetic dielectrics. Meanwhile, it should be mentioned that the heat convection between the heated sample and environment also affects the heat transfer in microwave processing of materials, but it presents much smaller influence compared with heat radiation.
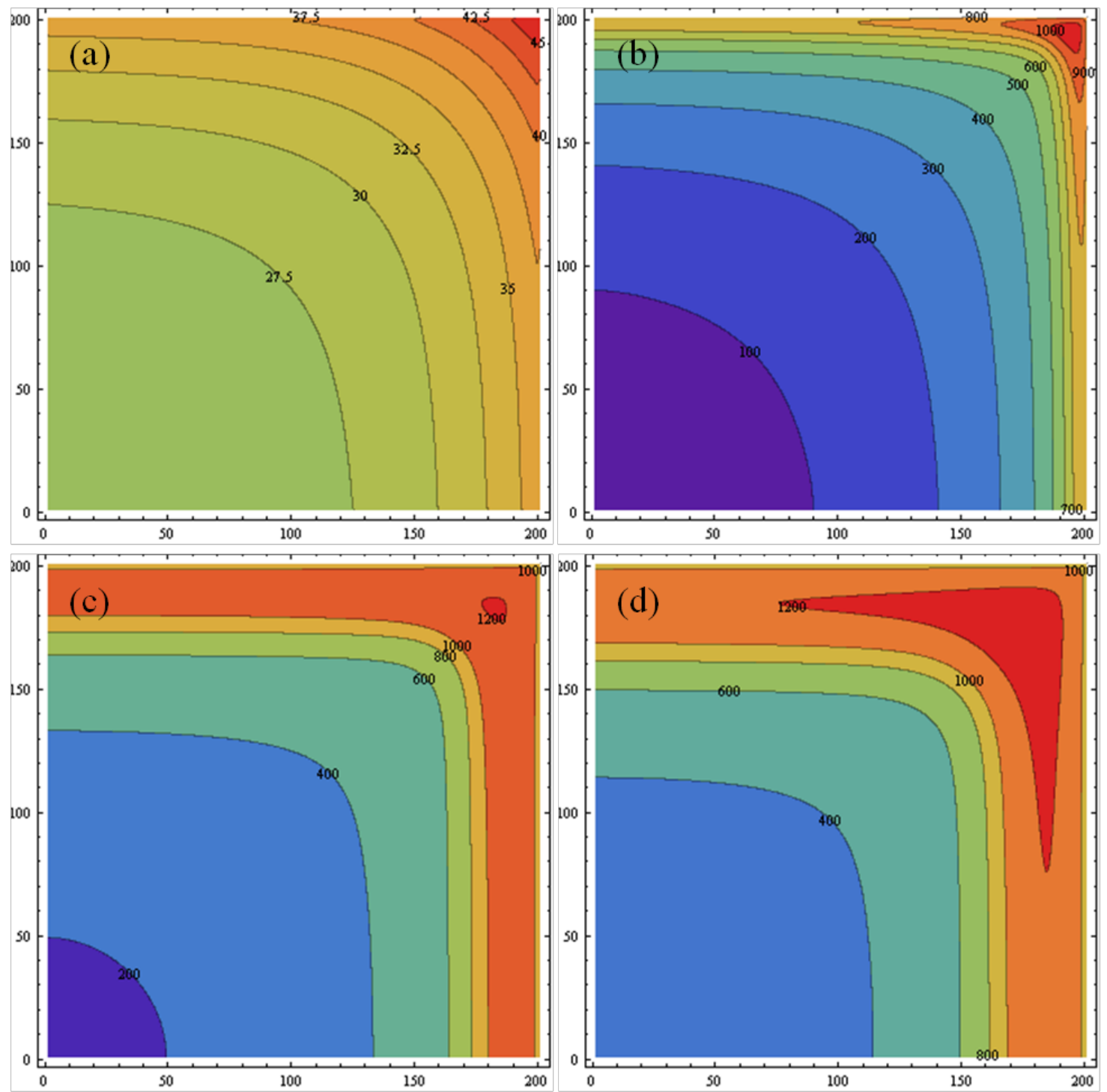

Fig. 7.10. Temperature $\left({ }^{\circ} \mathrm{C}\right)$ profiles in the 2-D object for different microwave heating

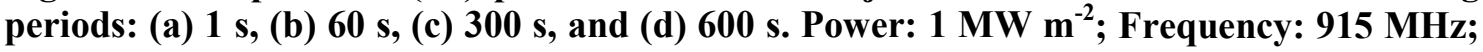
Dimension $(L): 0.2 \mathrm{~m}$. 


\subsubsection{Effect of Heating Power}

The temperature profiles of the object for different microwave powers in the range of 0.5 to $4 \mathrm{MW} \mathrm{m}^{-2}$ are presented in Fig. 7.11. The temperature of the object increases with increasing microwave power. The highest temperatures are $711^{\circ} \mathrm{C}, 1042{ }^{\circ} \mathrm{C}, 1144{ }^{\circ} \mathrm{C}$, and $1228{ }^{\circ} \mathrm{C}$ (not shown in Fig. 7.11) for $0.5 \mathrm{MW} \mathrm{m}^{-2}, 1 \mathrm{MW} \mathrm{m}^{-2}, 2 \mathrm{MW} \mathrm{m}^{-2}$, and 4 MW $\mathrm{m}^{-2}$, respectively. The temperature inside the object also increases apparently as the power level increases. As indicated in Fig. 7.11, the area with the temperature below $400{ }^{\circ} \mathrm{C}$ diminishes in a large amount after the power increases from 0.5 to $4 \mathrm{MW} \mathrm{m} \mathrm{m}^{-2}$. Moreover, it is interesting to note that these highest temperatures locate at different mesh positions: $(197,197),(194,194),(191,191)$, and $(189,189)$, respectively. This indicates the hot spot shifts towards the center of the object. In fact, a higher power input results in a larger temperature increase during the same heating time. The contribution to temperature increase from microwave generation becomes even considerable compared with other factors, especially heat diffusion. Although the heat radiation is quite strong at high temperatures, the diffusion also becomes slower due to smaller diffusivity at high temperatures. The hot spot locates at the position closer to the center of the object to keep heat balance during microwave heating. 

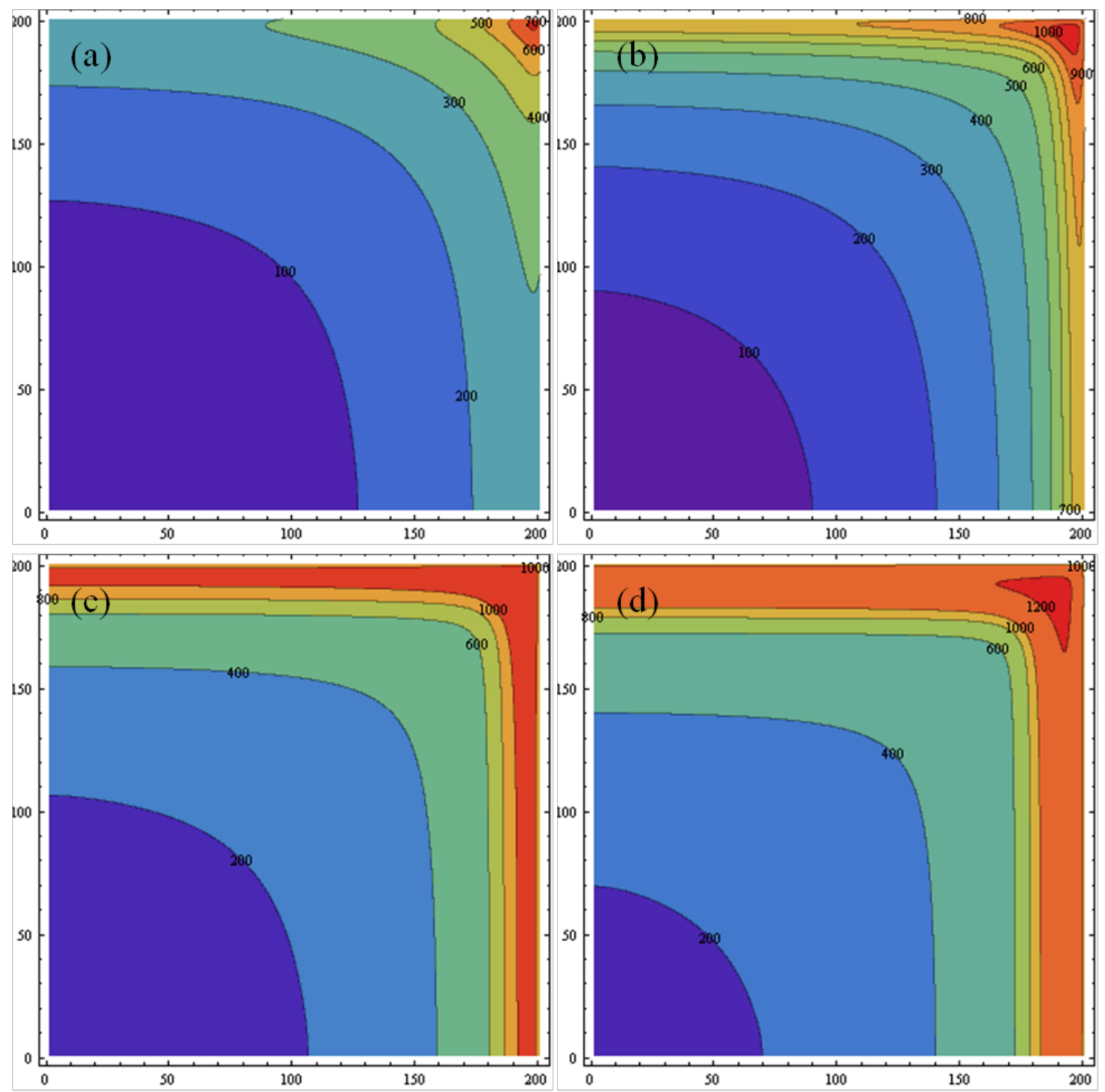

Fig. 7.11. Temperature $\left({ }^{\circ} \mathrm{C}\right)$ profiles in the 2-D object under different microwave heating powers: (a) $0.5 \mathrm{MW} \mathrm{m}$, (b) $1 \mathrm{MW} \mathrm{m}^{-2}$, (c) $2 \mathrm{MW} \mathrm{m}^{-2}$, and (d) $4 \mathrm{MW} \mathrm{m}^{-2}$. Heating time: 60 s; Frequency: $915 \mathrm{MHz}$; Dimension $(L): 0.2 \mathrm{~m}$.

\subsubsection{Effect of Microwave Frequency}

It is known that two frequencies, 915 and $2450 \mathrm{MHz}$, are commonly used in the microwave processing of materials. To evaluate the effect of microwave frequency on 
the temperature distribution in magnetic dielectrics, the temperature profiles in the object for different microwave heating periods at a frequency of $2450 \mathrm{MHz}$ is shown in Fig. 7.12 with a comparison with $915 \mathrm{MHz}$ in Fig. 7.10. A careful comparison indicates that the temperature increase rate at $2450 \mathrm{MHz}$ is much higher than that at $915 \mathrm{MHz}$ in the outer layer of the object and the heating rate is in agreement with the experimental measurement. ${ }^{203}$ Conversely, in the inner section of the sample, a lower temperature at $2450 \mathrm{MHz}$ is observed compared with $915 \mathrm{MHz}$. The difference also increases as microwave heating continues. This observation can be ascribed to the variation of dielectric and magnetic properties of magnetic dielectrics under microwave irradiation. These properties are strongly dependent on the microwave frequency and temperature. Such dependence can be indicated by the change in microwave penetration depth $\left(D_{p}\right)$ in magnetic dielectrics where the permeability cannot be ignored.

According to eq. (3.22), it is obvious that the penetration depth at $915 \mathrm{MHz}$ is larger than that at $2450 \mathrm{MHz}$ in a broad temperature range due to the much longer wavelength $\left(\lambda_{0}\right)$ at $915 \mathrm{MHz}$. However, it should be noted that the temperature-dependent dielectric and magnetic parameters (permittivity and permeability, respectively) also influence the penetration depth. ${ }^{65}$. The microwave penetration depth at $2450 \mathrm{MHz}$ is found to be much smaller than that at $915 \mathrm{MHz}$ below $500{ }^{\circ} \mathrm{C}$. This indicates, under the same conditions (power, heating time, object dimension, etc.), most of microwave energy at $2450 \mathrm{MHz}$ would dissipate in the area closer to surface than that at $915 \mathrm{MHz}$. With increasing heating time, the temperature of object increases and the radiation effect at 
the surface of materials grows rapidly. In other words, more energy is emitted (lost) into surrounding at $2450 \mathrm{MHz}$ than $915 \mathrm{MHz}$. Hence, the temperature of the object inner section under the same power level at $2450 \mathrm{MHz}$ is much lower than that at $915 \mathrm{MHz}$. Microwave heating at $915 \mathrm{MHz}$ thus exhibits better heating uniformity.
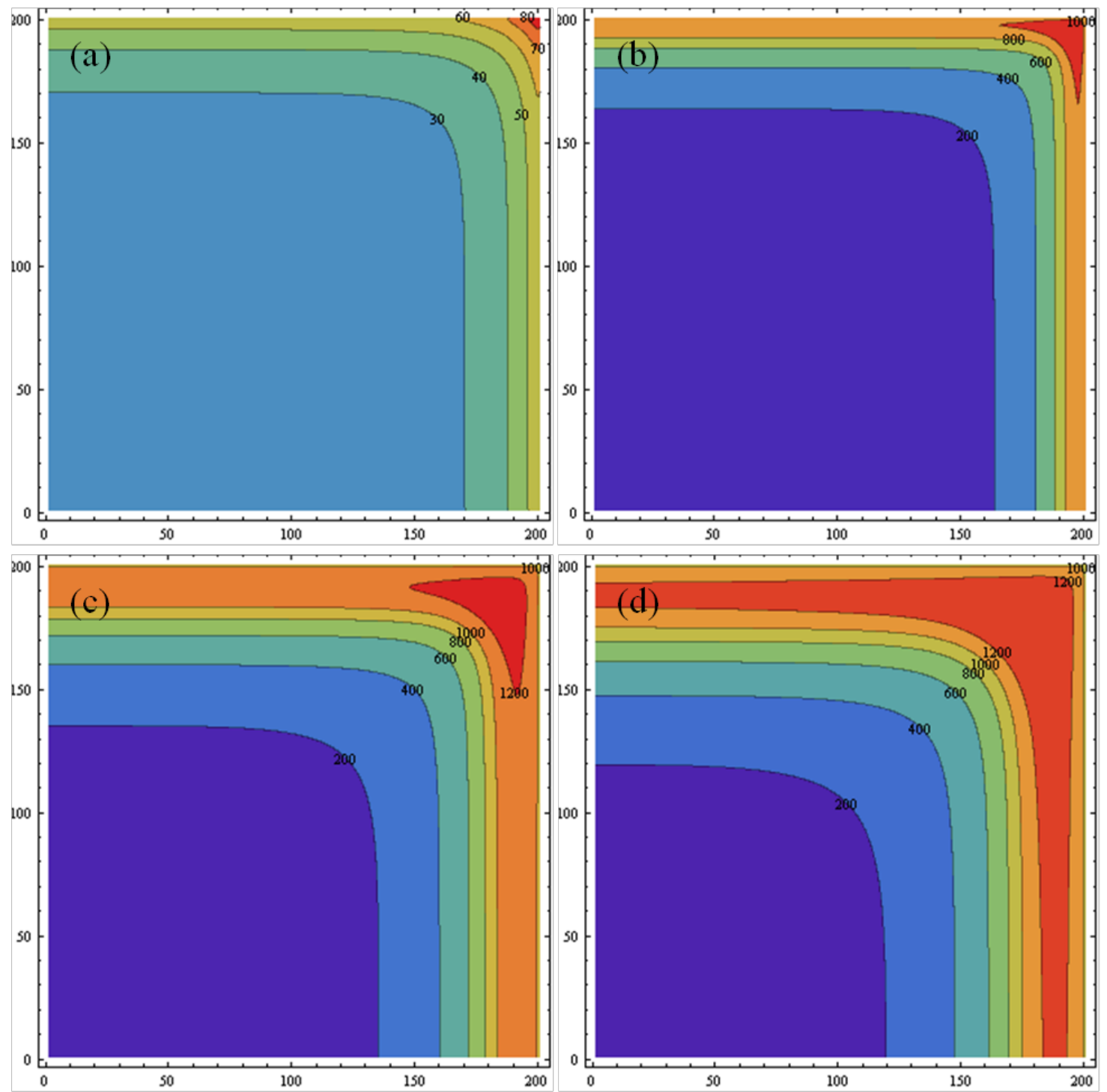

Fig. 7.12. Temperature $\left({ }^{\circ} \mathrm{C}\right)$ profiles in the 2-D object for different microwave heating

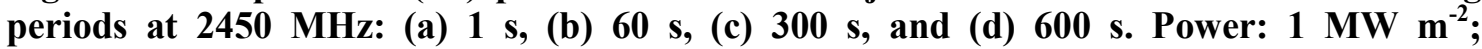
Dimension $(L): 0.2 \mathrm{~m}$. 


\subsubsection{Effect of Object Dimension}

Unlike the conventional thermal heating, relatively rapid uniform heating is much easier to achieve in microwave heating mainly due to the longer microwave penetration depth, and thus deeper microwave penetration in the materials. However, an obvious temperature gradient could also be expected provided the materials dimension is much larger than penetration depth, as shown in Fig. 7.13, where the temperature distributions for the object with different dimensions $(L=0.2 \mathrm{~m}, 0.15 \mathrm{~m}, 0.1 \mathrm{~m}$, and $0.05 \mathrm{~m}$, respectively) are displayed. The temperature homogeneity in the object improves as the dimension is reduced. The highest temperature area expands and moves close to the center of object. With continuous heating (e.g., to $300 \mathrm{~s}$ ), it is predicted that the overall body temperature would increases continuously. Because the penetration depth is determined by the microwave frequency and the materials response represented by both permittivity and permeability, it is better and more convenient to adjust the dimension of the magnetic dielectrics in microwave heating. Additionally, because of the much lower temperature of the surrounding area, the temperature of the internal block would be higher than that of the outer layer of the block. This accounts for the experimental observation in microwave heating, heat transfers from object center to the surface, which is opposite to the direction of heat transfer in conventional heating. ${ }^{1,65}$

However, it is expected that a further decrease in the dimension size (e.g., $L=0.02 \mathrm{~m})$ would result in an apparent "standing wave" phenomenon, which may dramatically 
worsen the heating uniformity. Under such conditions, the influence of microwave reflection at the interface between the magnetic dielectric and surrounding has to be considered and Maxwell's equations rather than Lambert's law should be used for the simulation.
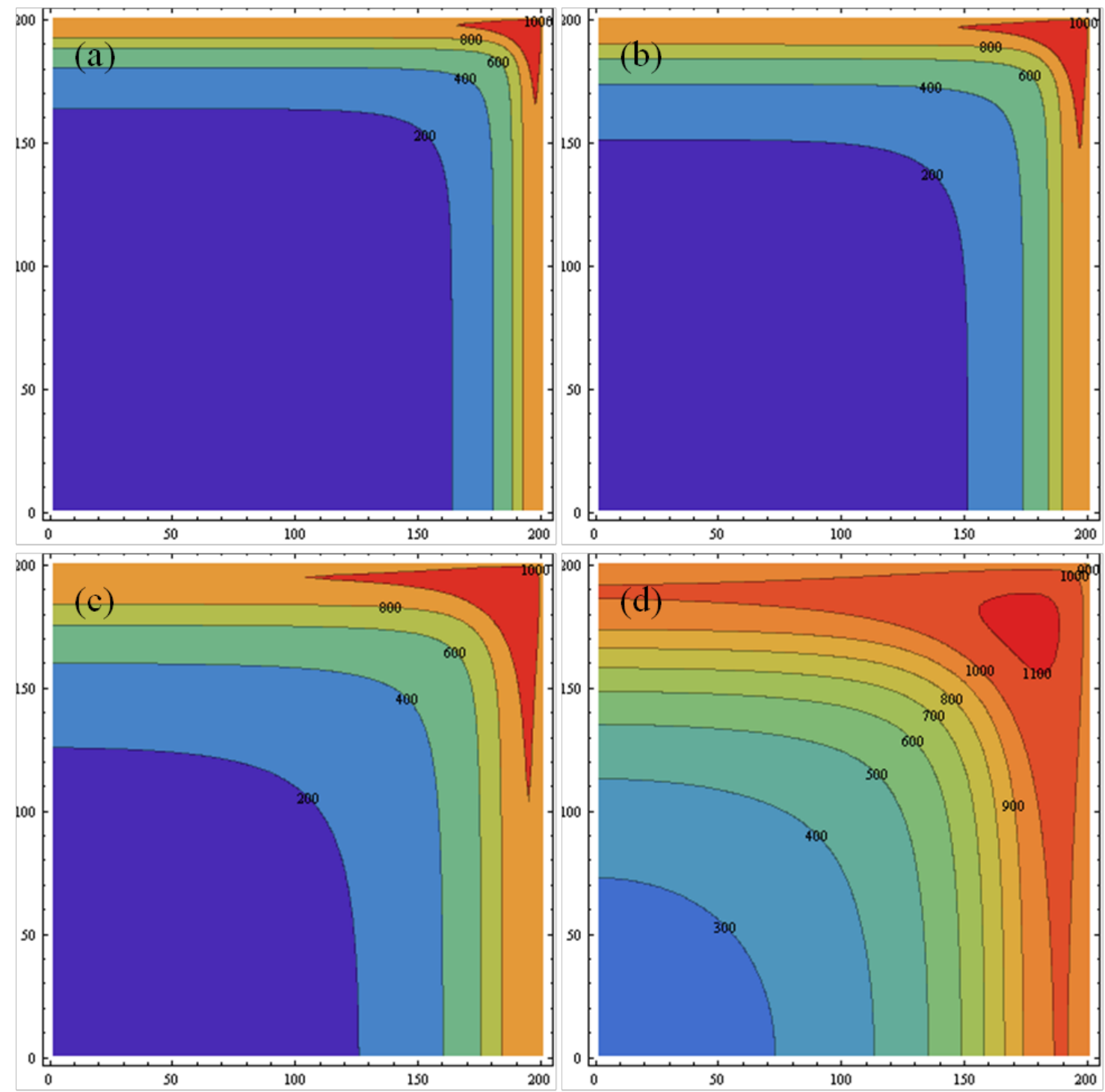

Fig. 7.13. Temperature $\left({ }^{\circ} \mathrm{C}\right)$ profiles in the 2-D object with different dimensions: (a) $0.2 \mathrm{~m}$,

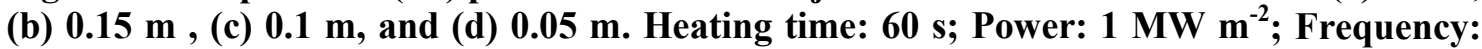
2450 MHz. 
The temperature profiles above demonstrate that heating time, microwave power, microwave frequency and object dimension affect the temperature distribution in the material. To obtain high heating performance and to avoid/minimize thermal runaway resulting from temperature nonuniformity, all these factors influencing the heat transfer in microwave processing of materials need to be considered. 


\title{
Chapter 8 Dimension Optimization for Absorbers in
}

\author{
Microwave Heating ${ }^{\dagger \dagger}$
}

\subsection{Effect of Absorber Dimension on Microwave Heating}

Microwave heating has shown its distinguishing characteristics such as volumetric nature compared with conventional methods, leading to extremely broad applications in materials heating and processing. The efficiency of microwave heating was found to be dependent on various factors, such as microwave power, ${ }^{128}$ frequency, ${ }^{199,209}$ radiation time, ${ }^{199}$ applicator design, ${ }^{199}$ sample microwave absorption properties and position as well as dimension, ${ }^{77,200,210}$ etc. Among these parameters, the effect of sample dimension was generally neglected by researchers in microwave processing of materials. In fact, although numerous studies have been focused on the utilization of microwave energy to assist materials heating and processing, few reports were released to investigate the effect of sample dimension. ${ }^{42,211}$ This is because all the mentioned factors influence

\footnotetext{
\# The content of this chapter was accepted for publication in TMS Annual Meeting, 4th International Symposium on High-Temperature Metallurgical Processing - Held During the TMS 2013 Annual Meeting and Exhibition ${ }^{208}$ by Zhiwei Peng, Jiann-Yang Hwang, Byoung-Gon Kim, Matthew Andriese, Xinli Wang.

Reproduced with permission from TMS Annual Meeting, 4th International Symposium on HighTemperature Metallurgical Processing - Held During the TMS 2013 Annual Meeting and Exhibition: Zhiwei Peng, Jiann-Yang Hwang, Byoung-Gon Kim, Matthew Andriese, Xinli Wang. Microwave Reflection loss of Ferric Oxide. TMS Annual Meeting, 4th International Symposium on HighTemperature Metallurgical Processing - Held During the TMS 2013 Annual Meeting and Exhibition. 2013; to be published. Copyright (C) 2013 The Minerals, Metals and Materials Society. (See Appendix F-1 for copyright permission).
} 
microwave heating and generally exert interactive effects to each other during the heating process, making clarification of the effect of sample dimension difficult. Therefore, it is quite necessary to quantify the effect of absorber dimension on microwave heating, advancing the understanding of microwave heating mechanism.

In the present study, the microwave dissipation behavior and heat transfer process in microwave absorbers were analyzed and the concept of reflection loss was introduced to optimize the absorber dimension in microwave heating. In order to show the importance of the dimension effect on microwave heating, hematite $\left(\mathrm{Fe}_{2} \mathrm{O}_{3}\right)$ was employed to determine the variation of reflection loss with thickness at widely used microwave frequencies based on the corresponding dielectric characterizations.

\subsection{Microwave Heat Generation and Heat Transfer}

Microwave heating is closely associated with the propagation and dissipation of microwaves in materials, which are normally described mathematically by Maxwell's equations with corresponding boundary conditions. The microwave field distributions in an absorber are defined by these equations and their variations are attributed to heating efficiency. Because of this characteristic, the first point that has to be considered for increasing microwave heating efficiency is to maximize the microwave energy absorption. 
From Poynting's theorem, ${ }^{133}$ the microwave energy absorption or heat generation $(P)$ in an absorber due to the microwave-matter interaction can be given as

$$
P=\pi f\left(\varepsilon_{0} \varepsilon_{r}|E|^{2}+\mu_{0} \mu_{r}^{\prime \prime}|H|^{2}\right)
$$

where $f$ is the microwave frequency, $\varepsilon_{0}$ and $\mu_{0}$ are the dielectric permittivity and magnetic permeability of free space, $\varepsilon_{r}{ }^{\prime \prime}$ and $\mu_{r}{ }^{\prime \prime}$ are the imaginary part of complex relative permittivity (dielectric loss factor) and the imaginary part of complex relative permeability (magnetic loss factor), and $|E|$ and $|H|$ denote the electric and magnetic fields inside the sample, respectively.

According to eq. (8.1), it is seen that the heat generation due to microwave irradiation is directly related to microwave frequency, microwave absorption properties (permittivity and permeability), and microwave field distribution. It seems that microwave heating is independent of absorber dimension. However, careful inspection would find that heat generation expressed in this equation does not consider the dynamic process of microwave propagation and dissipation in materials, whose effects are rather important in large-scale microwave heating and processing of materials.

Additionally, microwave heating efficiency not only depends on the heat generation within the absorber but also the heat transfer inside the absorber during the heating process. The increase in temperature of the absorber under microwave irradiation can be determined from the heat transfer differential equation [considering $x$ direction in this case, see eq. (7.1)]. ${ }^{199}$ The equation shows that the increase in temperature at given 
heating time $(\partial T / \partial t)$ depends on both heat conduction and microwave heat generation. With consideration of specific heat transfer boundary conditions, the final temperature within the absorber can be determined. However, the heat transfer process during microwave heating may give rise to nonuniform temperature distributions in large-scale materials, which have been observed by many researchers. ${ }^{212-214}$ Those inhomogeneous temperature distributions may further lead to some serious problems in microwave heating, such as the formation of hot spot and occurrence of thermal runaway, which make the heating out of control. ${ }^{62,63}$ Obviously, these problems are closely relevant to the dimension of the microwave absorber since the heat transfer rate in the absorber is relatively slow compared with the oscillation rate of microwave electric and magnetic fields. Considering this effect, the primary purpose of this study would be to increase the microwave absorption of the sample with an optimal dimension, and also to improve the microwave heating uniformity.

\subsection{Dimension Optimization using Reflection Loss}

In order to meet these two conditions, a parameter must be introduced to efficiently evaluate the dimension effect. Prior scientists employed the microwave penetration depth $\left(D_{p}\right)$ to show the microwave dissipation along the direction of microwave propagation ${ }^{65}$ It is defined as the distance from the surface into an absorber at which the traveling wave power drops to $e^{-1}(e=2.718)$ from its value at the surface. For an absorber having both dielectric loss and magnetic loss, the penetration depth can be 
calculated by eq. (3.22) ${ }^{65,140}$ Microwave power dissipates very quickly along the depth of the material in a penetration depth, beyond which only slow energy losses occur with increasing dimension. This suggests that an absorber having a dimension large than $D_{p}$ may have a nonuniform power (heat) distribution, resulting in inhomogeneous temperatures within the material. To address this problem, some researchers have proposed that the dimension (thickness) of an absorber under one-sided irradiation can be limited to a penetration depth. ${ }^{65}$ This would give high microwave absorption and also diminish the nonuniform temperature distribution. However, it does not consider the situation in most microwave heating, where the sample is loaded in a metallic applicator. Therefore, a suitable estimation of optimal dimension for microwave absorbers needs to take into account the reflection of microwaves in the cavity.

Regarding the microwave heating condition illustrated in Fig. 8.1, an extensive exploration of literature indicates that calculation of reflection loss (RL) at the interface between air and the metal-backed sample may be employed to investigate the effect of absorber dimension (thickness) on microwave heating. ${ }^{215-218}$ According to the transmission line theory, RL can be calculated using the following equation: ${ }^{215}$

$$
R L=20 \log \frac{\left|\sqrt{\frac{\mu_{r}}{\varepsilon_{r}}} \tanh \left(j \frac{2 \pi f}{c} \sqrt{\mu_{r} \varepsilon_{r}} d\right)-1\right|}{\left|\sqrt{\frac{\mu_{r}}{\varepsilon_{r}}} \tanh \left(j \frac{2 \pi f}{c} \sqrt{\mu_{r} \varepsilon_{r}} d\right)+1\right|},
$$

where $\mu_{r}$ and $\varepsilon_{r}$ are the complex relative permeability and permittivity, respectively, of the microwave absorber, $j$ is the imaginary unit, $c$ is the velocity of microwaves in free 
space, and $d$ is the thickness of the absorber. This parameter has been widely used in the analysis of microwave absorption properties of electromagnetic wave absorbers to overcome electromagnetic interference (EMI) shielding problems. ${ }^{216-218}$ However, no prior study has been devoted to apply it in microwave heating and to discuss the effect of absorber dimension on the microwave heating efficiency.

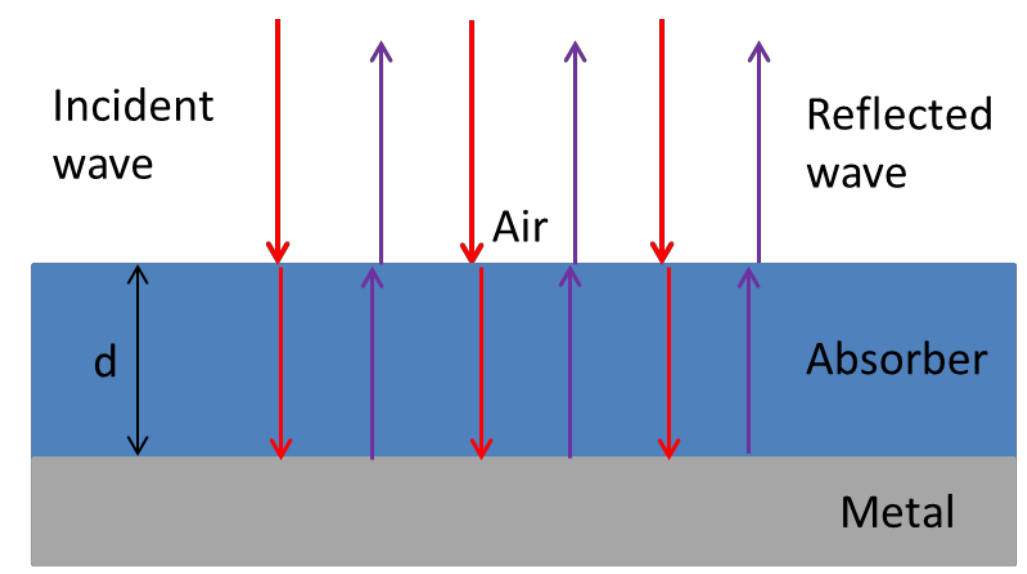

Fig. 8.1. Schematic of an absorber under microwave irradiation.

As shown by eq. (8.2), RL deals with microwave attenuation with consideration of permittivity, permeability, microwave frequency and microwave reflection. The calculated losses for absorbers at commonly used frequencies, mainly 915 and 2450 $\mathrm{MHz}$, over a broad temperature range probably provide useful information for the optimization of absorber dimension. To clarify this application, hematite $\left(\mathrm{Fe}_{2} \mathrm{O}_{3}\right)$ was taken for the microwave absorption characterizations in this work (also see Chapter 5). 
The dielectric parameters of hematite (compacted powders with a bulk density of $2.79 \mathrm{~g}$ $\mathrm{cm}^{-3}$ ) were measured using the cavity perturbation technique, ${ }^{65}$ and the results are shown in Fig. 8.2(a). The real and imaginary parts of complex relative permittivity $\left(\varepsilon_{r}{ }^{\prime}\right.$ and $\left.\varepsilon_{r}{ }^{\prime \prime}\right)$ of hematite slightly increase with temperature below $450{ }^{\circ} \mathrm{C}$, above which $\varepsilon_{r}{ }^{\prime}$ increases significantly while $\varepsilon_{r}$ " presents a broad dielectric loss peak between 450 and $1000{ }^{\circ} \mathrm{C}$. This leads to the formation of a broad dielectric loss tangent $\left(\tan \delta_{e}=\varepsilon_{r}{ }^{\prime \prime} / \varepsilon_{r}{ }^{\prime}\right)$ peak between 450 and $1000{ }^{\circ} \mathrm{C}$ at both frequencies, as illustrated in Fig. 8.2(b).
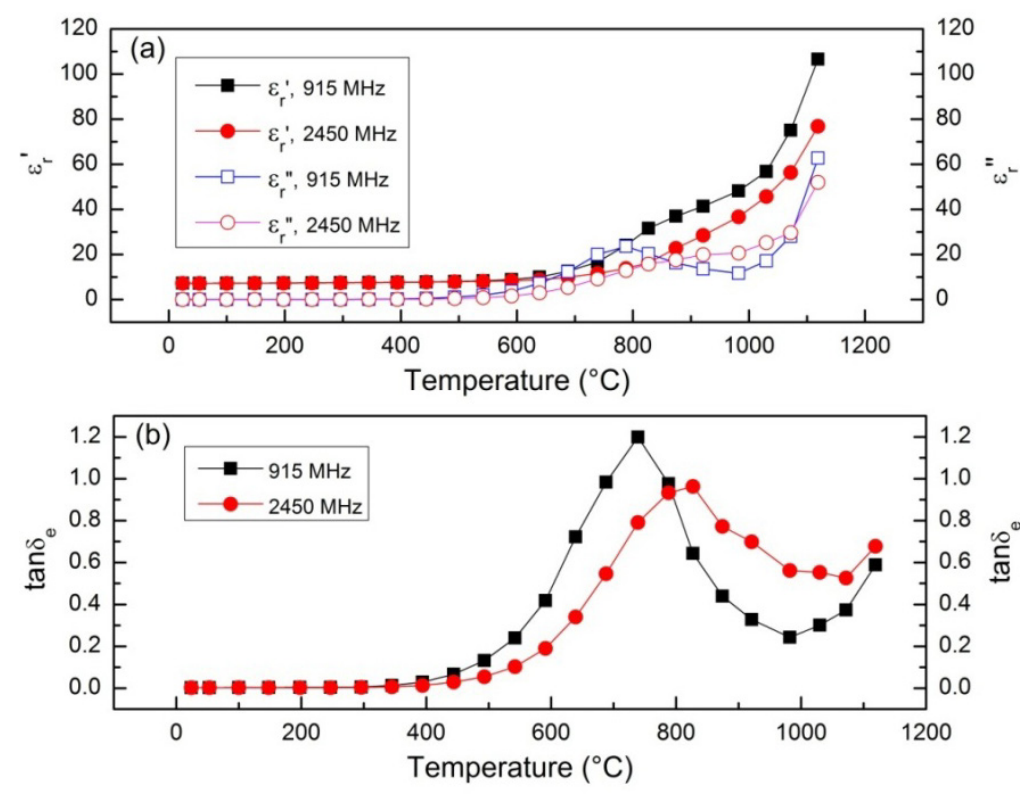

Fig. 8.2. (a) Complex relative permittivity of hematite vs. temperature at 915 and 2450 MHz. (b) Dielectric loss tangent of hematite vs. temperature at 915 and $2450 \mathrm{MHz}$. 
Based on the measurements RL of hematite can be determined using eq. (8.2). Considering hematite having very weak magnetism in the measured temperature range, ${ }^{145,146}$ unit complex relative permeability $\left(\mu_{r}=1\right)$ was taken in the calculation of reflection loss.

Figure 8.3(a) shows the calculated results of RL versus temperature for hematite with increasing sample thickness (from 0.005 to $0.05 \mathrm{~m}$ ) at $915 \mathrm{MHz}$. The sample with a thickness of $0.005 \mathrm{~m}$ exhibits a negligible absorption peak (RL trough) in the whole temperature range, suggesting it having rather weak microwave absorption under this condition. The intensity of the absorption peak increases as the thickness increases from 0.005 to $0.03 \mathrm{~m}$. It then decreases as the thickness increases from 0.03 to $0.05 \mathrm{~m}$. The maximum peak reaches $-38.46 \mathrm{~dB}$ at $\sim 600{ }^{\circ} \mathrm{C}$ as the sample has a thickness of $0.03 \mathrm{~m}$. The temperature range of the maximum absorption peak with RL below $-10 \mathrm{~dB}(>90 \%$ microwave absorption) is found to be $250{ }^{\circ} \mathrm{C}$ (the difference between 450 and $700{ }^{\circ} \mathrm{C}$ ). This shows that hematite of $0.03 \mathrm{~m}$ thickness can be heated rapidly at $915 \mathrm{MHz}$ above $450{ }^{\circ} \mathrm{C}$. 

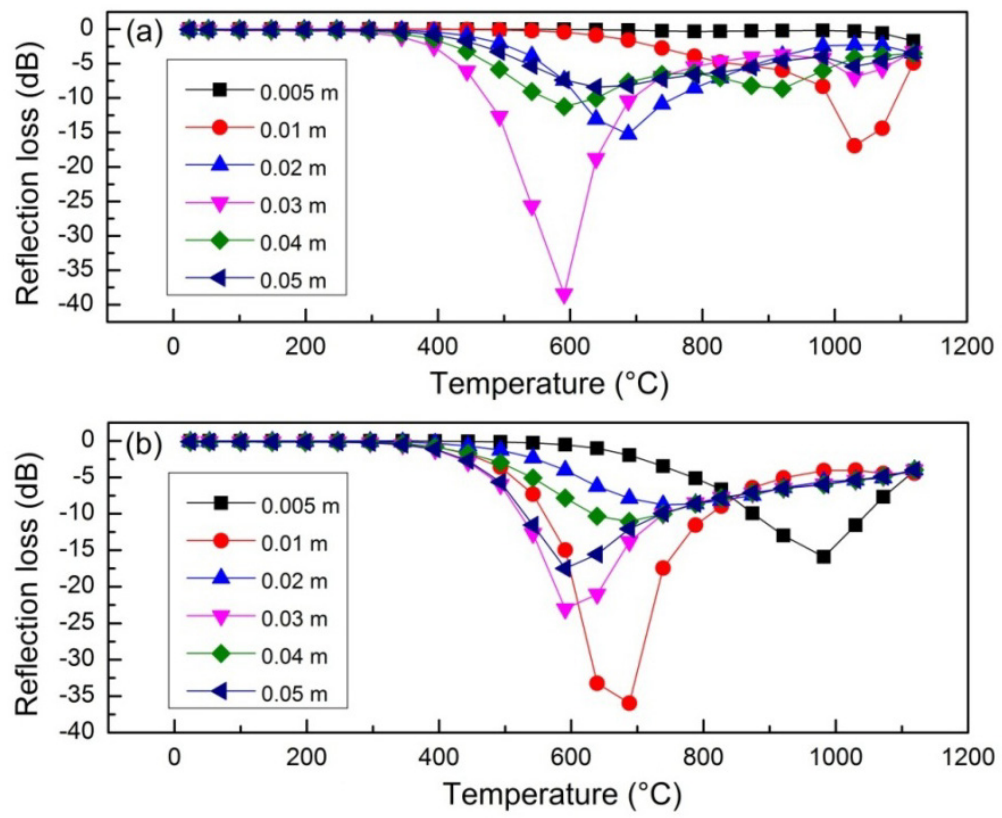

Fig. 8.3. High-temperature microwave absorption of hematite: (a) Calculated reflection loss vs. temperature at $915 \mathrm{MHz}$. (b) Calculated reflection loss vs. temperature at 2450 MHz.

Figure 8.3(b) shows the calculated results of RL versus temperature for hematite with increasing sample thickness (from 0.005 to $0.05 \mathrm{~m}$ ) at $2450 \mathrm{MHz}$. It is seen that the variation trend of reflection loss at $2450 \mathrm{MHz}$ is similar to that at $915 \mathrm{MHz}$. The maximum peak reaches $-35.97 \mathrm{~dB}$ at $\sim 700{ }^{\circ} \mathrm{C}$ as the sample has a thickness of $0.01 \mathrm{~m}$. The temperature range of the maximum absorption peak with RL below $-10 \mathrm{~dB}$ is 250 ${ }^{\circ} \mathrm{C}$ (the difference between 550 and $800{ }^{\circ} \mathrm{C}$ ), which is the same as that at $915 \mathrm{MHz}$. The only difference can be noted is that the absorption peak is shifted by $100{ }^{\circ} \mathrm{C}$ to higher temperatures. This shift is in exact agreement with the variation of dielectric loss tangent at the measured frequencies, as presented in Fig. 8.2(b). It indicates that the 
maximum absorption peak position of hematite is determined by the dielectric loss tangent.

From these results, it is observed that the microwave absorption of hematite presents a strong thickness dependence during heating. The maximum absorption peak indicated by RL shows that high microwave absorption in the material can be achieved in a wide temperature range. This meets the requirement for the first condition for optimal microwave heating. Also, it considers the variation of microwave dielectric properties with temperature, which has been ignored in most previous studies. Because this optimization of absorber dimension takes into account the whole heating temperature range, it minimizes the adverse effect of heat transfer during heating. Thus, it is easier to obtain relatively uniform microwave heating for absorbers with the optimal dimensions based on the analysis of reflection loss.

In summary, this study discusses the effect of absorber dimension on microwave heating. It shows that, to achieve high microwave heating efficiency, an optimal sample dimension needs to be determined via the analyses of microwave absorption and heat transfer in materials. Based on the characterization of microwave absorption properties, the calculated reflection loss over the temperature range of heating is found to be useful for obtaining a rapid optimization of the absorber dimension, which increases microwave absorption and achieves relatively uniform heating. 


\section{Chapter 9 Absorber Impedance Matching in Microwave}

\section{Heating}

\subsection{Perfect Impedance Matching}

As mentioned before, it is critical to reduce and suppress microwave reflection from the sample surface during microwave heating. To achieve this purpose, RL was used to optimize the sample dimension for absorbers in last chapter. The results clearly showed that it is useful for obtaining a rapid optimization of absorber dimension by determining $\mathrm{RL}$ in the given dimension range. Consequently, a high performance of microwave heating can be expected. However, it should be emphasized that the highest absorption of an absorber during the whole heating process is still unavailable without a general design formula or rule for the heating, which depends on the dielectric and magnetic parameters and matching thickness.

In fact, the search for a generalized design rule based on the calculation of reflection loss for optimizing microwave absorbing materials has been the subject of research for

\footnotetext{
${ }_{\ddagger}$ The content of this chapter was previously published in Applied Physics Express ${ }^{219}$ by Zhiwei Peng, Jiann-Yang Hwang, Matthew Andriese.

Reproduced with permission from Applied Physics Express: Zhiwei Peng, Jiann-Yang Hwang, Matthew Andriese. Absorber Impedance Matching in Microwave Heating. Applied Physics Express. 2012;5(7):077301-077301-3. Copyright (C) 2012 The Japan Society of Applied Physics. (See Appendix G-1 for copyright permission).
} 
a long time. ${ }^{220-224}$ To reduce the reflection loss in the materials, the concept of impedance matching was introduced. It was originally developed for designing the input impedance of an electrical load in electrical engineering to minimize the power reflection from the load. Over the past few years, several approaches were proposed to obtain the optimum microwave absorber structure by considering the effect of microwave bandwidth. ${ }^{225-235}$ However, there is no detailed study on the influence of the heating process in which heating temperature and heat transfer play important roles in the design; no general design rule for the absorber in microwave heating is yet available.

The present work is aimed to achieve the maximum microwave absorption in materials in microwave heating by developing a new mathematical function for designing absorbers having perfect impedance matching. The impedance matching degrees of a representative microwave absorber, hematite, in a broad temperature range up to $\sim 1000$ ${ }^{\circ} \mathrm{C}$ were subsequently calculated using the derived function. It is demonstrated that this function can be used to obtain perfect impedance matching for materials, achieving the highest absorption in the entire microwave heating process.

To attain the maximum absorption by using impedance matching, the microwave reflection should be as low as possible $(\mathrm{RL} \rightarrow-\infty)$. According to the definition of $\mathrm{RL}$ for a single-layer microwave absorber backed by a metal plate, when RL approaches $-\infty$, the following condition should be satisfied: 


$$
\sqrt{\frac{\mu_{r}}{\varepsilon_{r}}} \tanh \left(j \frac{2 \pi f}{c} \sqrt{\mu_{r} \varepsilon_{r}} d\right)=1
$$

The above equation could be also written as

$$
\operatorname{coth}\left(j \frac{2 \pi f}{c} \sqrt{\mu_{r} \varepsilon_{r}} d\right)=\sqrt{\frac{\mu_{r}}{\varepsilon_{r}}},
$$

or

$$
\operatorname{coth}(\gamma d)=\sqrt{\frac{\mu_{r}}{\varepsilon_{r}}} .
$$

Apparently, eq. (9.3) shows that an absorber having a specific dimension or optimum microwave parameters (permittivity and permeability) can have the maximum absorption without serious microwave reflection, namely, perfect impedance matching.

\subsection{Derivation of the Function for Evaluating Impedance Matching}

In general, it is quite difficult to achieve perfect impedance matching in microwave heating. This is due to the fact that absorbers have the specific microwave permeability and permittivity at the given temperature and microwave frequency. The reflection loss may vary significantly under different conditions. This can be revealed by the calculated RL values for microwave heating of hematite, as illustrated in Chapter 8 . Although hematite presents a very low reflection (approximately $-40 \mathrm{~dB}$ ) at high temperatures, the microwave absorption is still weak at low temperatures. In such a 
case, it is more important to improve the microwave absorption by optimizing the matching dimension and dielectric and magnetic parameters.

To obtain perfect matching, we may introduce a function used for calculating the impedance matching degree of the absorber to further analyze the effects of dimension and microwave parameters on microwave heating. As shown in the previous chapters, the propagation constant $\gamma$ is generally expressed as

$$
\gamma=\alpha+\beta j
$$

Thus, the left-hand side of eq. (9.3) can be presented as

$$
\operatorname{coth}(\gamma d)=\operatorname{coth}[(\alpha+\beta j) d]
$$

Thus, we have

$$
\operatorname{coth}(\gamma d)=\frac{\exp ([2(\alpha+\beta j) d])+1}{\exp ([2(\alpha+\beta j) d])-1}
$$

Because the relationships to ordinary trigonometric functions can be given using Euler's formula for complex numbers, eq. (9.6) may be described as

$$
\operatorname{coth}(\gamma d)=\frac{\exp (2 \alpha d) \cos (2 \beta d)+j \exp (2 \alpha d) \sin (2 \beta d)+1}{\exp (2 \alpha d) \cos (2 \beta d)+j \exp (2 \alpha d) \sin (2 \beta d)-1}
$$

Finally, we have

$$
\operatorname{coth}(\gamma d)=\frac{\exp (4 \alpha d)-1-j 2 \exp (2 \alpha d) \sin (2 \beta d)}{\exp (4 \alpha d)-2 \exp (2 \alpha d) \cos (2 \beta d)+1}
$$

Similarly, the right-hand side of eq. (9.3) can be presented as 


$$
\sqrt{\frac{\mu_{r}}{\varepsilon_{r}}}=\sqrt{\frac{\mu_{r}{ }^{\prime}-j \mu_{r}{ }^{\prime} \tan \delta_{m}}{\varepsilon_{r}{ }^{\prime}-j \varepsilon_{r}{ }^{\prime} \tan \delta_{e}}} .
$$

The derivation of eq. (9.9) may be briefly described as follows:

$$
\begin{aligned}
& \sqrt{\frac{\mu_{r}}{\varepsilon_{r}}}=\sqrt{\frac{\mu_{r}{ }^{\prime}}{\varepsilon_{r}{ }^{\prime}}} \sqrt{\frac{\cos \delta_{e}\left(\cos \delta_{m}-j \sin \delta_{m}\right)}{\cos \delta_{m}\left(\cos \delta_{e}-j \sin \delta_{e}\right)}}, \\
& \sqrt{\frac{\mu_{r}}{\varepsilon_{r}}}=\sqrt{\frac{\mu_{r}{ }^{\prime}}{\varepsilon_{r}{ }^{\prime}}} \sqrt{\frac{\cos \delta_{e} \exp \left(-j \delta_{m}\right)}{\cos \delta_{m} \exp \left(-j \delta_{e}\right)}}, \\
& \sqrt{\frac{\mu_{r}}{\varepsilon_{r}}}=\sqrt{\frac{\mu_{r}{ }^{\prime}}{\varepsilon_{r}{ }^{\prime}} \frac{\cos \delta_{e}}{\cos \delta_{m}}} \exp \left(-j \frac{\delta_{m}-\delta_{e}}{2}\right), \\
& \sqrt{\frac{\mu_{r}}{\varepsilon_{r}}}=\sqrt{\frac{\mu_{r}{ }^{\prime}}{\varepsilon_{r}{ }^{\prime}} \frac{\cos \delta_{e}}{\cos \delta_{m}}}\left[\cos \left(\frac{\delta_{m}-\delta_{e}}{2}\right)-j \sin \left(\frac{\delta_{m}-\delta_{e}}{2}\right)\right] .
\end{aligned}
$$

By comparing eqs. (9.8) and (9.13), the following two equations should be satisfied simultaneously to obtain perfect impedance matching:

$$
\begin{aligned}
& \frac{\exp (4 \alpha d)-1}{\exp (4 \alpha d)-2 \exp (2 \alpha d) \cos (2 \beta d)+1}=\sqrt{\frac{\mu_{r}{ }^{\prime}}{\varepsilon_{r}{ }^{\prime}} \frac{\cos \delta_{e}}{\cos \delta_{m}}} \cos \left(\frac{\delta_{m}-\delta_{e}}{2}\right), \\
& \frac{2 \exp (2 \alpha d) \sin (2 \beta d)}{\exp (4 \alpha d)-2 \exp (2 \alpha d) \cos (2 \beta d)+1}=\sqrt{\frac{\mu_{r}{ }^{\prime}}{\varepsilon_{r}{ }^{\prime}} \frac{\cos \delta_{e}}{\cos \delta_{m}}} \sin \left(\frac{\delta_{m}-\delta_{e}}{2}\right) .
\end{aligned}
$$

Derivations of eqs. (9.14) and (9.15) lead to

$$
\begin{gathered}
\frac{\exp (4 \alpha d)-1}{2 \exp (2 \alpha d) \sin (2 \beta d)}=\cot \left(\frac{\delta_{m}-\delta_{e}}{2}\right), \\
\frac{\exp (2 \alpha d)}{2 \sin (2 \beta d)}-\frac{1}{2 \exp (2 \alpha d) \sin (2 \beta d)}=\cot \left(\frac{\delta_{m}-\delta_{e}}{2}\right),
\end{gathered}
$$




$$
\frac{1}{2} \exp (2 \alpha d) \csc (2 \beta d)-\frac{1}{2} \exp (-2 \alpha d) \csc (2 \beta d)=\cot \left(\frac{\delta_{m}-\delta_{e}}{2}\right) .
$$

Considering heat transfer in microwave heating, it is more advantageous to introduce the penetration depth, $D_{p}$, into eq. (9.18). Thus, it can be transformed as

$$
\frac{1}{2} \exp \left(\frac{d}{D_{p}}\right) \csc (2 \beta d)-\frac{1}{2} \exp \left(-\frac{d}{D_{p}}\right) \csc (2 \beta d)=\cot \left(\frac{\delta_{m}-\delta_{e}}{2}\right) .
$$

Here, a function, $f(d)$, may be used to show the impedance matching degree of the absorber in microwave heating. It can be expressed as

$$
f(d)=\frac{1}{2} \exp \left(\frac{d}{D_{p}}\right) \csc (2 \beta d)-\frac{1}{2} \exp \left(-\frac{d}{D_{p}}\right) \csc (2 \beta d)-\cot \left(\frac{\delta_{m}-\delta_{e}}{2}\right) .
$$

For the absorber without magnetic loss (dielectrics), eq. (9.20) is simplified as

$$
f(d)=\frac{1}{2} \exp \left(\frac{d}{D_{p}}\right) \csc (2 \beta d)-\frac{1}{2} \exp \left(-\frac{d}{D_{p}}\right) \csc (2 \beta d)+\cot \left(\frac{\delta_{e}}{2}\right) .
$$

Also, according to the definitions of $D_{p}$ and $\beta$, the following equation can be derived:

$$
\beta=\frac{1}{2 D_{p}} \cot \left(\frac{\delta_{e}}{2}\right) \text {. }
$$

Thus, from eqs. (9.21) and (9.22), we have

$$
\begin{aligned}
& f(d)=\frac{1}{2} \exp \left(\frac{d}{D_{p}}\right) \csc \left[\frac{d}{D_{p}} \cot \left(\frac{\delta_{e}}{2}\right)\right] \\
& -\frac{1}{2} \exp \left(-\frac{d}{D_{p}}\right) \csc \left[\frac{d}{D_{p}} \cot \left(\frac{\delta_{e}}{2}\right)\right]+\cot \left(\frac{\delta_{e}}{2}\right) .
\end{aligned}
$$


As revealed by eq. (9.23), an appropriate value of $\delta_{e}$ has to be found in order to attain perfect impedance matching [indicated by $f(d) \rightarrow 0$ ] for an absorber having a certain dimension (indicated by $d / D_{p}$ ), and vice versa.

\subsection{Perfect Impedance Matching Map}

According to eq. (9.23), the relation between $f(d)$ and $d / D_{p}$ and $\delta_{e}$ can be obtained, as presented in Fig. 9.1. The impedance matching map in Fig. 9.1 shows that there are possible solutions of the function $f(d)$, depending on the values of $d / D_{p}$ and $\delta_{e}$. It can be further confirmed by the matching patterns from the top viewpoint (Fig. 9.2), right viewpoint (Fig. 9.3), and front viewpoint (Fig. 9.4).

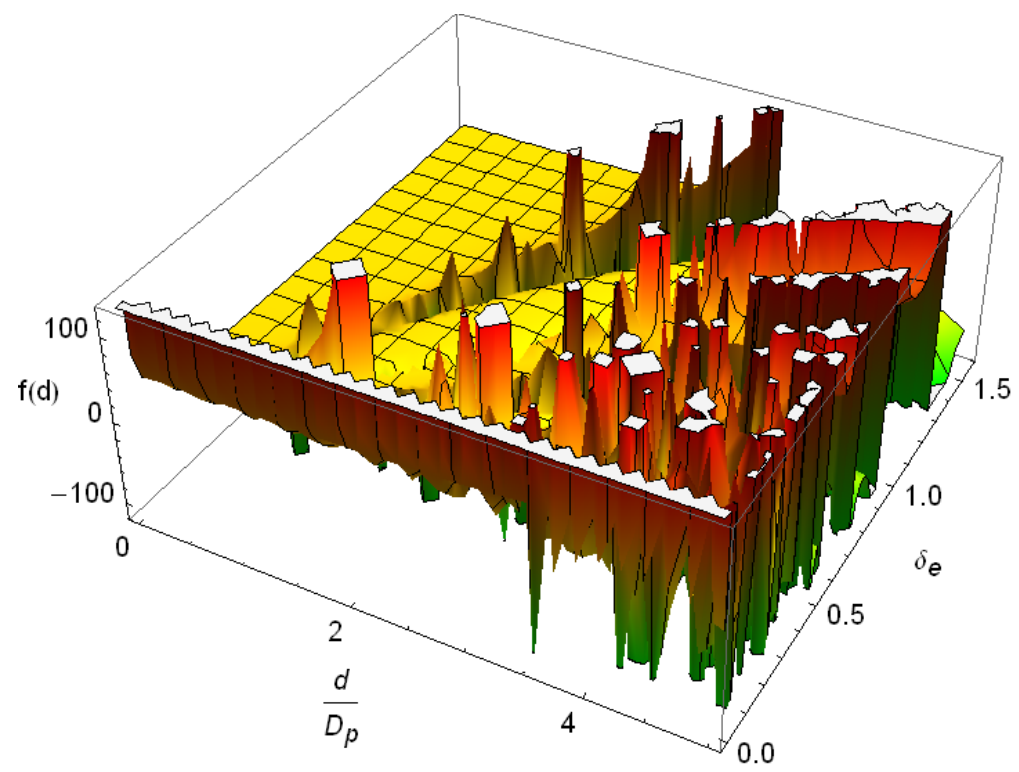

Fig. 9.1. Impedance matching map for a dielectric absorber (3-D view). 


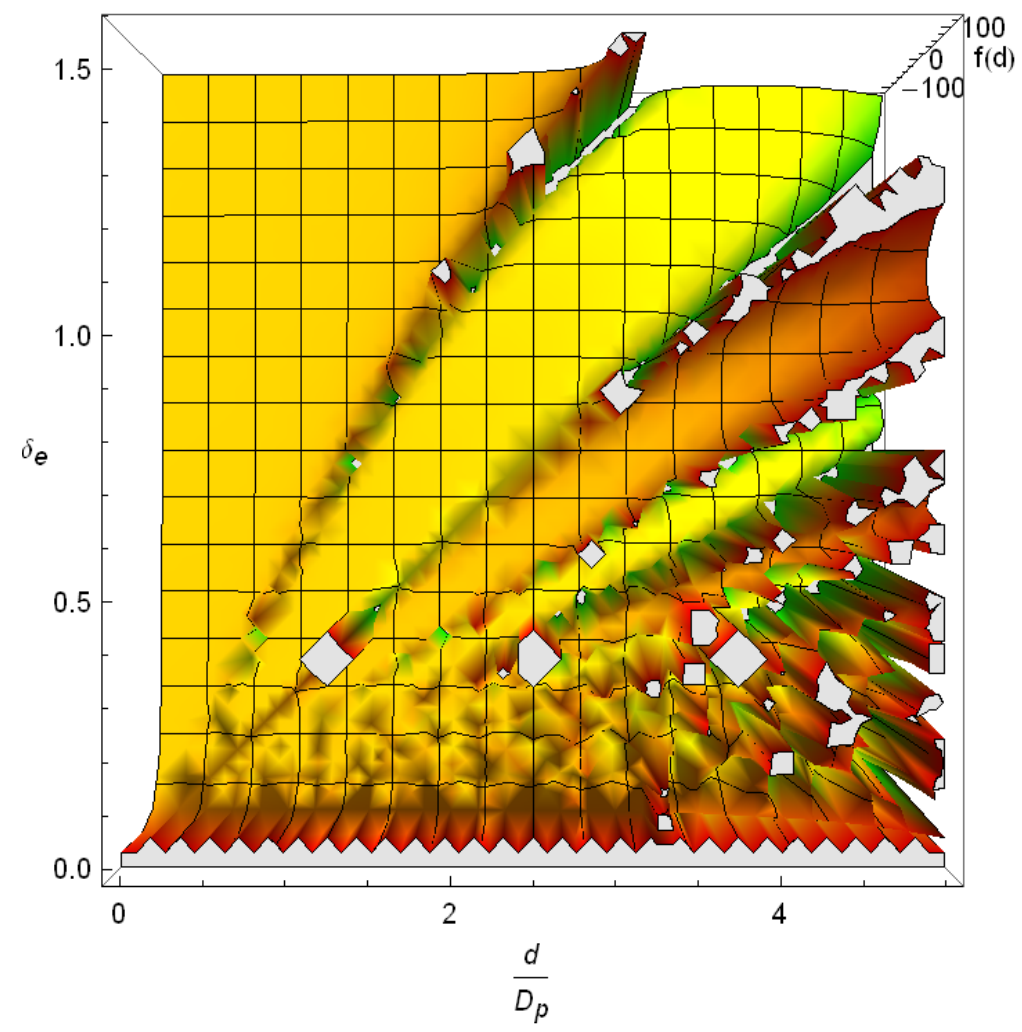

Fig. 9.2. Impedance matching map for a dielectric absorber (top viewpoint).

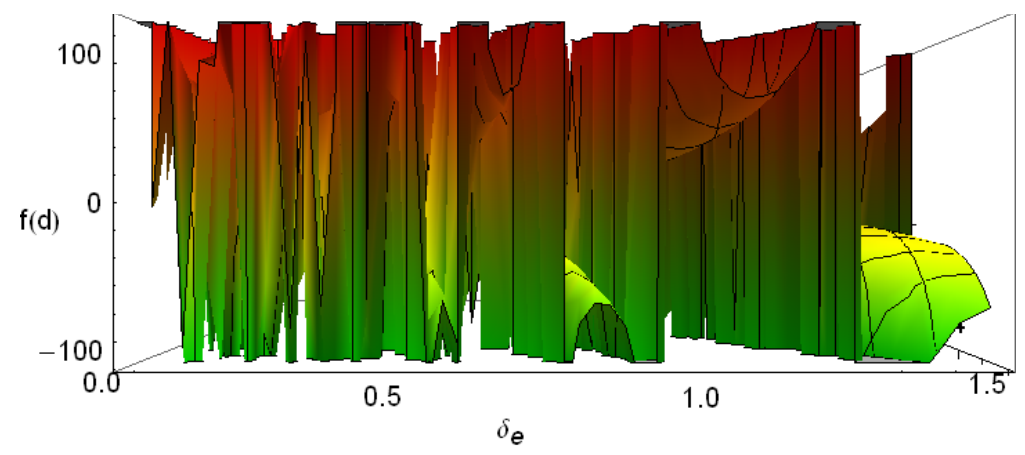

Fig. 9.3. Impedance matching map for a dielectric absorber (right viewpoint). 


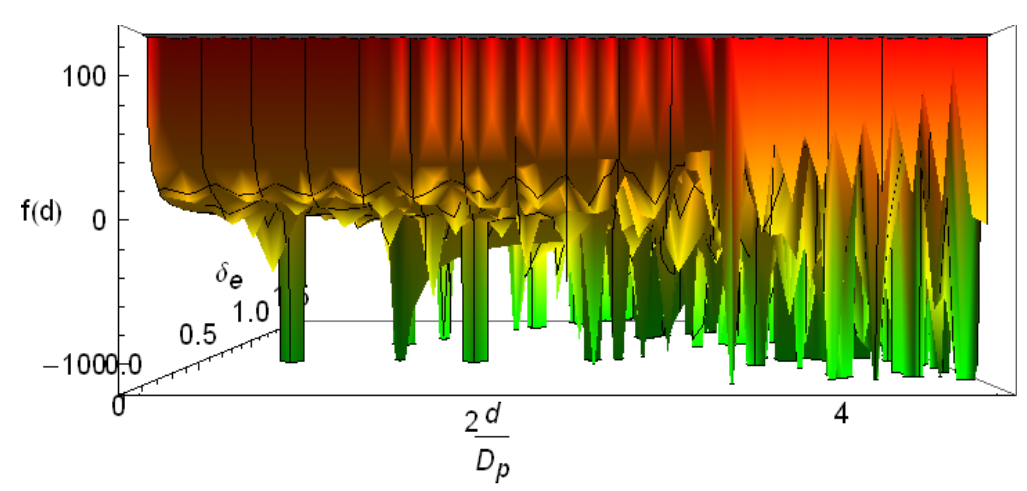

Fig. 9.4. Impedance matching map for a dielectric absorber (front viewpoint).

\subsubsection{Effect of $\delta_{e}$}

The effect of $\delta_{e}$ on the impedance matching can be demonstrated by the solutions of $f(d)$ with given loss tangent angles. Figures 9.5-9.14 show the possible solutions of the function with the $\delta_{e}$ values of $\pi / 24, \pi / 12, \pi / 6, \pi / 4$, and $3 \pi / 8$, respectively. It is indicated that there are numerous solutions of the function when the loss tangent phase angle is small. For example, it is easy to find a solution for perfect impedance matching in the $d / D_{p}$ range of $0-3.3$ when $\delta_{e}$ is $\pi / 24$. However, as the $\delta_{e}$ value increases, fewer solutions of the function can be observed in the given $d / D_{p}$ range. For instance, when the $\delta_{e}$ values are $\pi / 12$ and $\pi / 6$, the possible solutions are only available in the $d / D_{p}$ ranges of $0-2.4$ and $0-1.6$, respectively. Further increase in $\delta_{e}$ leads to no solution of the function (e.g., $\delta_{e}=\pi / 4$ or $3 \pi / 8$ ), as presented in Figs. 9.11-9.14. This clearly shows that it is impossible to gain the complete microwave absorption for absorbers having the $\delta_{e}$ values beyond $\pi / 4$. The maximum thickness of materials, which corresponds to 
perfect impedance matching, decreases with increasing dielectric loss tangent phase angle.

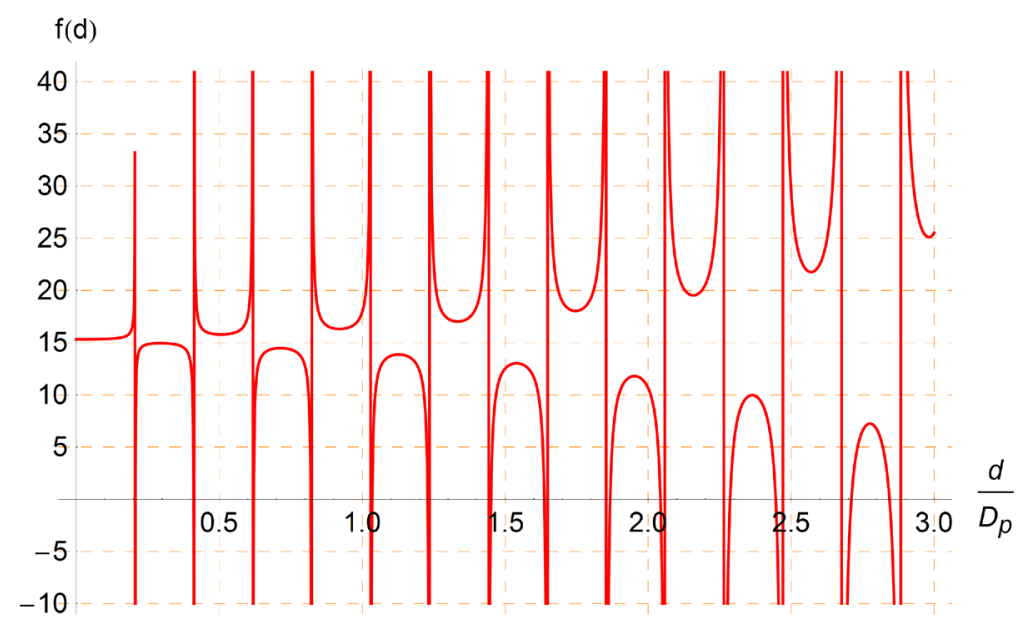

Fig. 9.5. $f(d)$ vs. $d / D_{p}$ ranging from 0 to $3\left(\delta_{e}=\pi / 24\right)$.

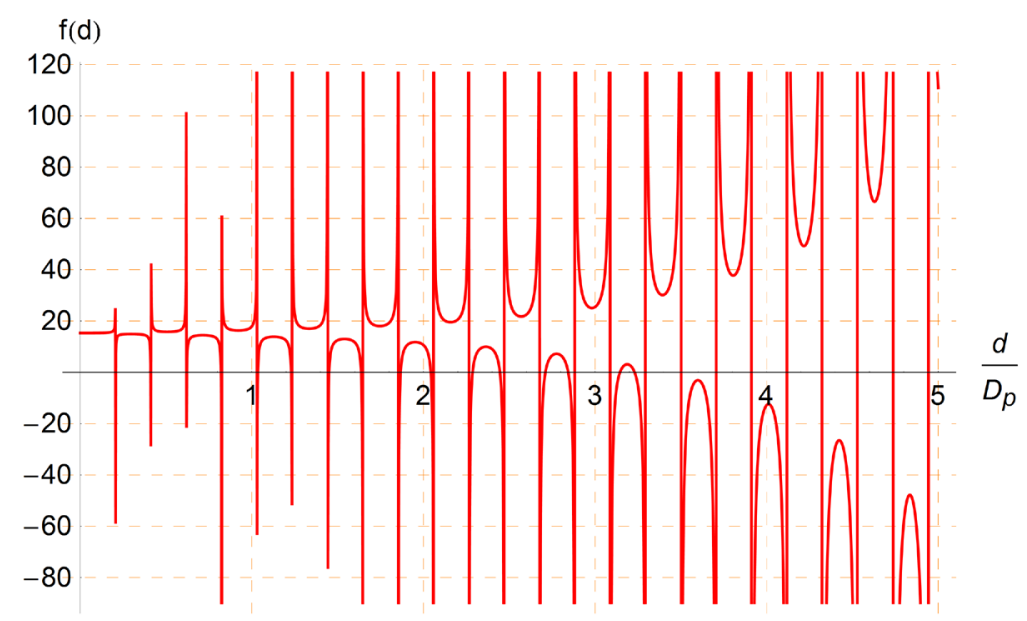

Fig. 9.6. $f(d)$ vs. $d / D_{p}$ ranging from 0 to $5\left(\delta_{e}=\pi / 24\right)$. 


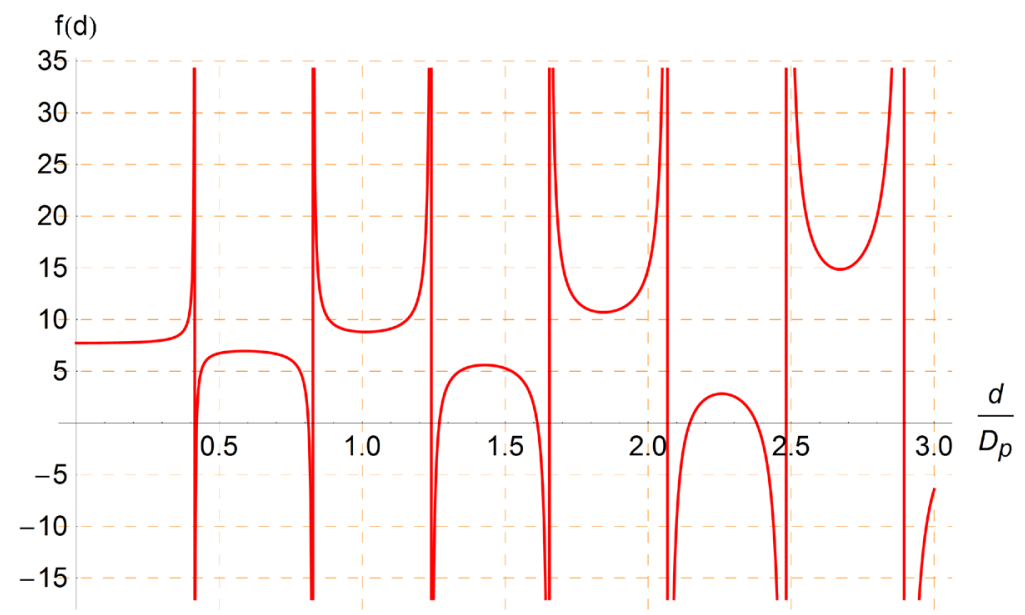

Fig. 9.7. $f(d)$ vs. $d / D_{p}$ ranging from 0 to $3\left(\delta_{e}=\pi / 12\right)$.

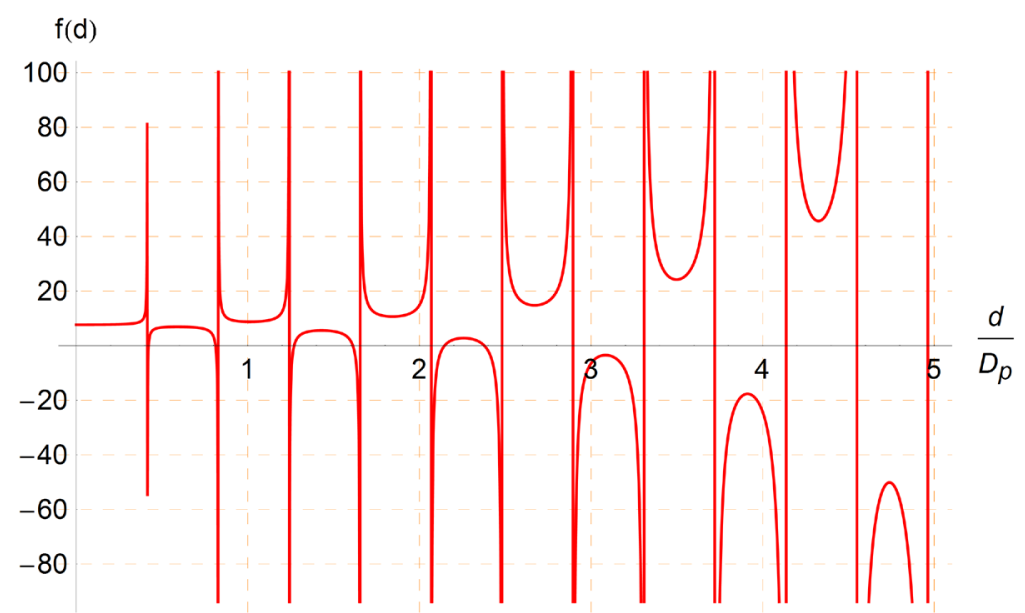

Fig. 9.8. $f(d)$ vs. $d / D_{p}$ ranging from 0 to $5\left(\delta_{e}=\pi / 12\right)$. 


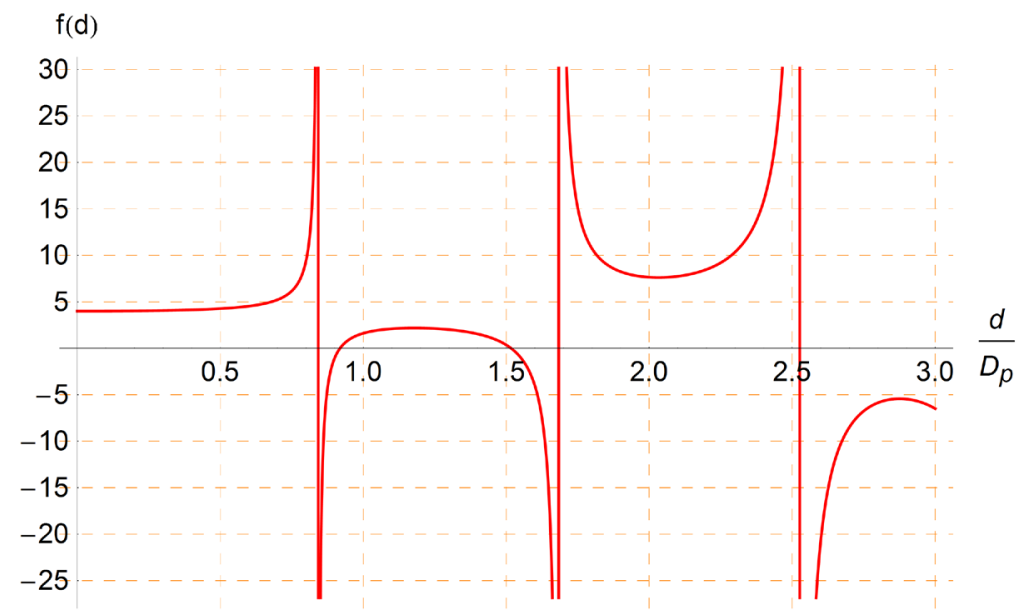

Fig. 9.9. $f(d)$ vs. $d / D_{p}$ ranging from 0 to $3\left(\delta_{e}=\pi / 6\right)$.

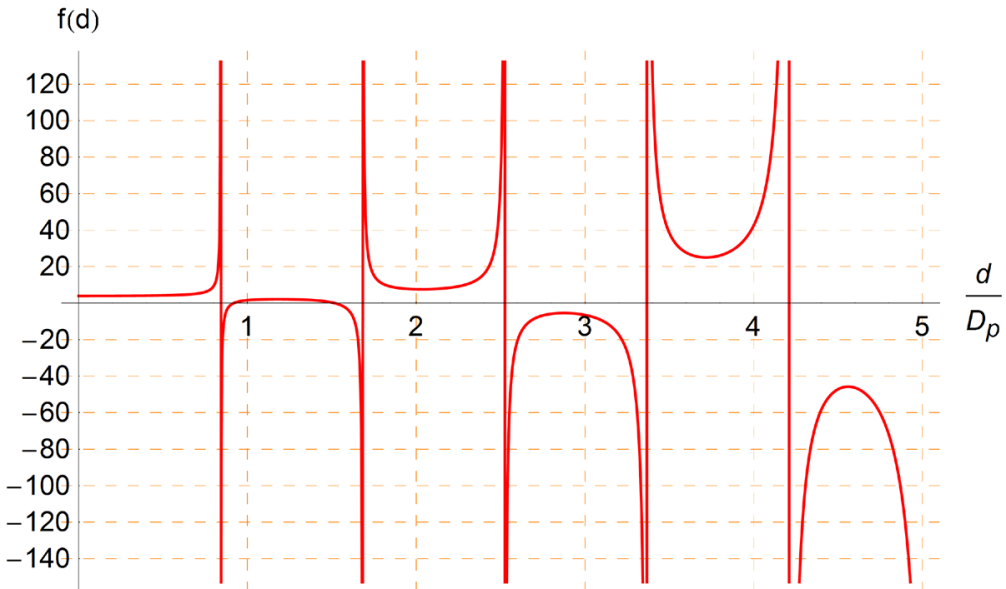

Fig. 9.10. $f(d)$ vs. $d / D_{p}$ ranging from 0 to $5\left(\delta_{e}=\pi / 6\right)$. 


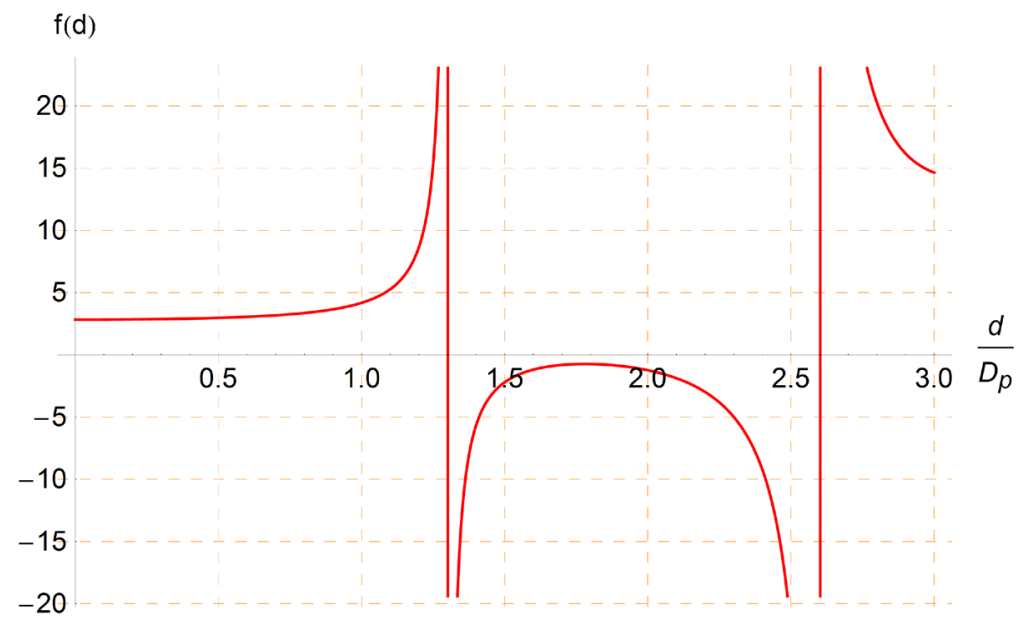

Fig. 9.11. $f(d)$ vs. $d / D_{p}$ ranging from 0 to $3\left(\delta_{e}=\pi / 4\right)$.

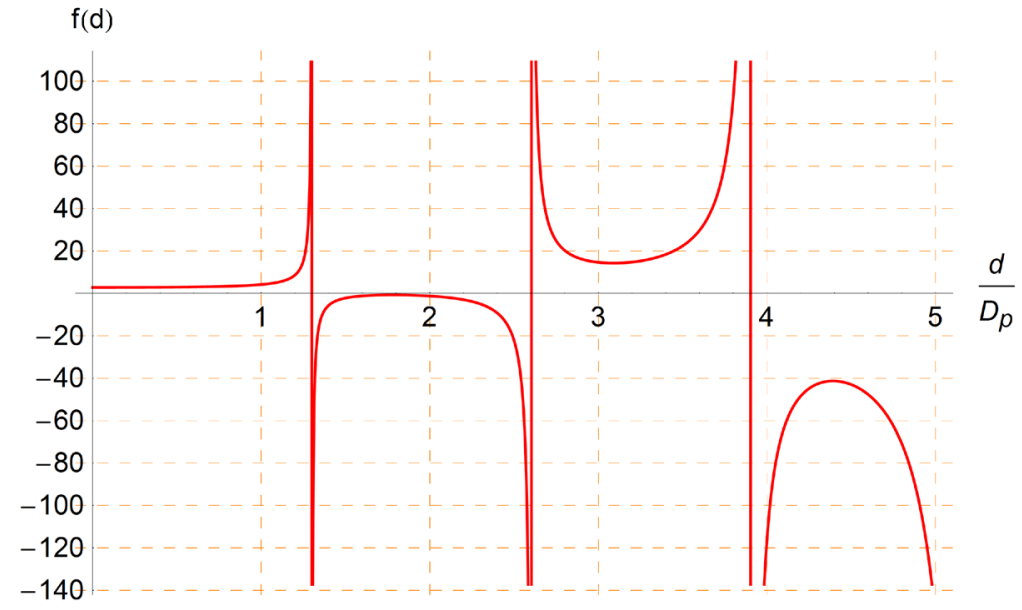

Fig. 9.12. $f(d)$ vs. $d / D_{p}$ ranging from 0 to $5\left(\delta_{e}=\pi / 4\right)$. 


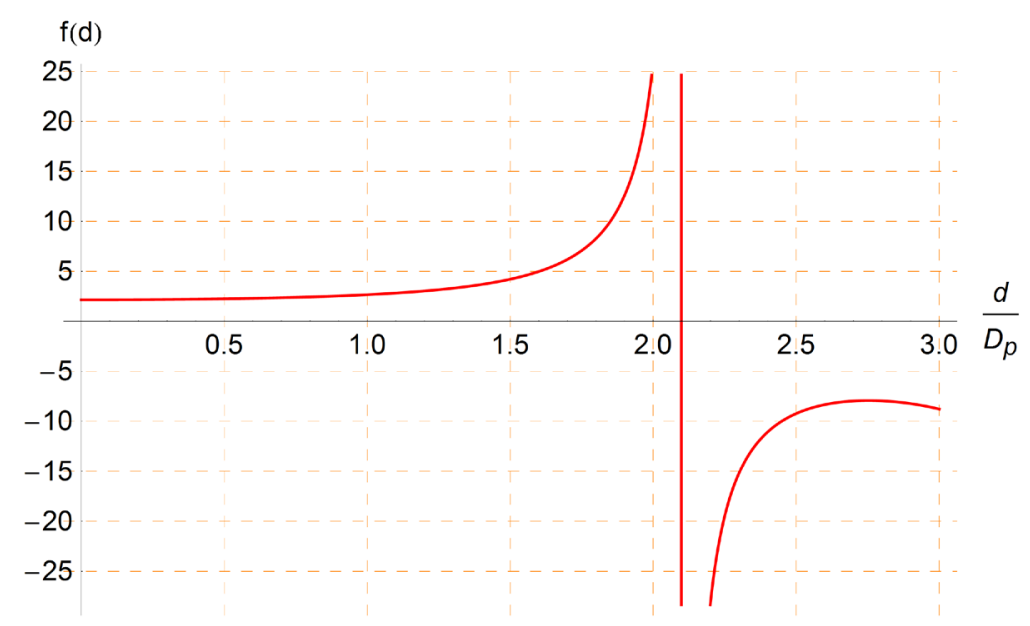

Fig. 9.13. $f(d)$ vs. $d / D_{p}$ ranging from 0 to $3\left(\delta_{e}=3 \pi / 8\right)$.

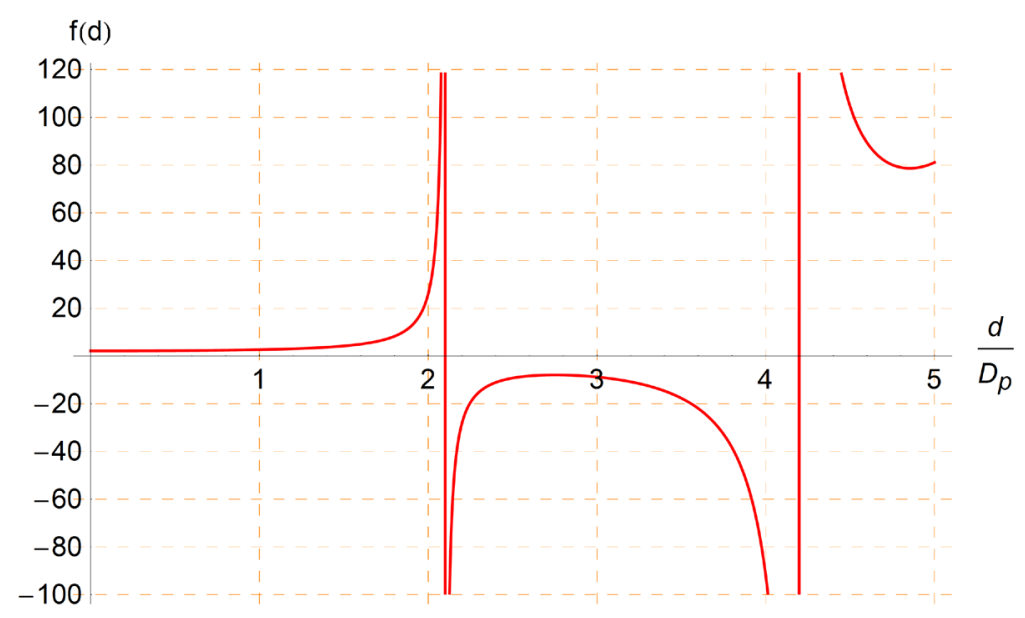

Fig. 9.14. $f(d)$ vs. $d / D_{p}$ ranging from 0 to $5\left(\delta_{e}=3 \pi / 8\right)$.

\subsubsection{Effect of $d / D_{p}$}

The effect of $d / D_{p}$ on the impedance matching is demonstrated by the solutions of the function $f(d)$ with the given values of $\delta_{e}$. Figures 9.15-9.24 display the possible solutions of the function as $d / D_{p}$ increases from 1 to 5 . When the values of $d / D_{p}$ are 1,2 , 
and 3 , it is easy to find solutions of $f(d)$ with the $\delta_{e}$ values below $0.6,0.4$, and 0.2 , respectively. Hence, it can be concluded that the optimum matching thickness increases with decreasing dielectric loss tangent phase angle, which agrees well with the finding indicated by the study on the effect of $\delta_{e}$.

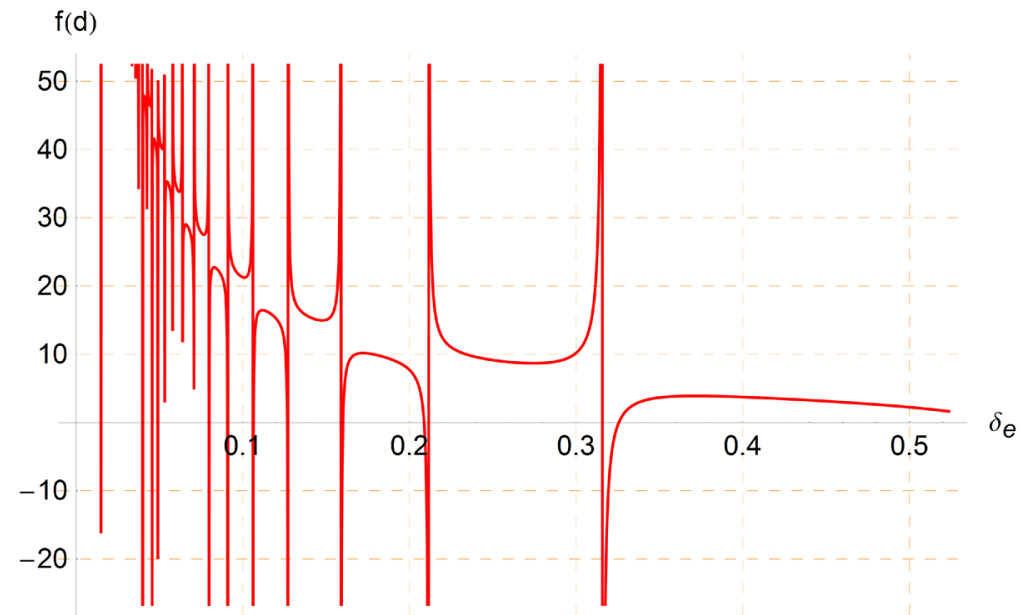

Fig. 9.15. $f(d)$ vs. $\delta_{e}$ ranging from 0 to $\pi / 6\left(d / D_{p}=1\right)$.

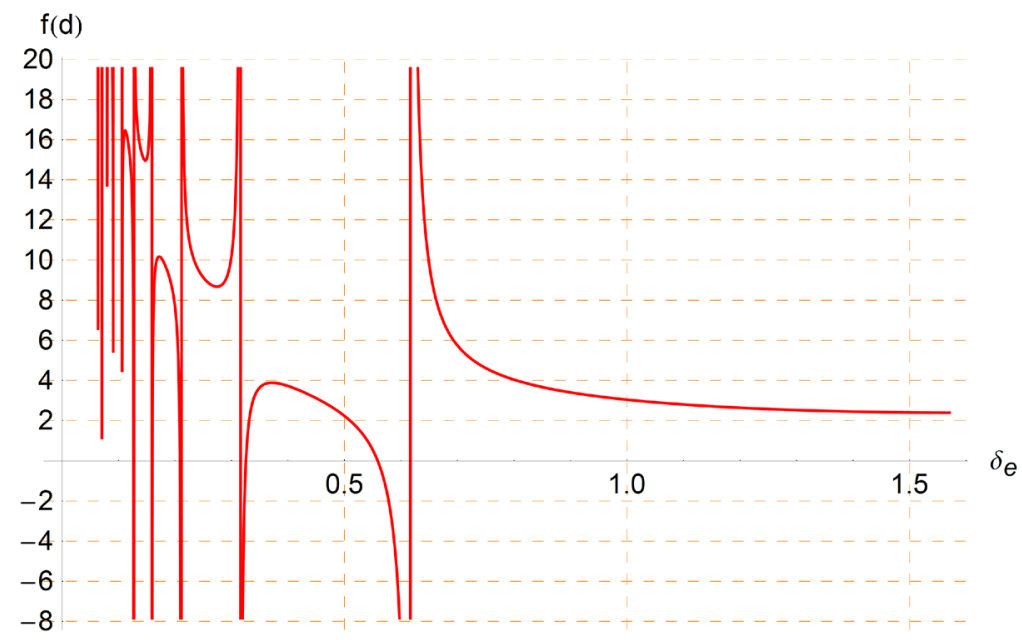

Fig. 9.16. $f(d)$ vs. $\delta_{e}$ ranging from 0 to $\pi / 2\left(d / D_{p}=1\right)$. 


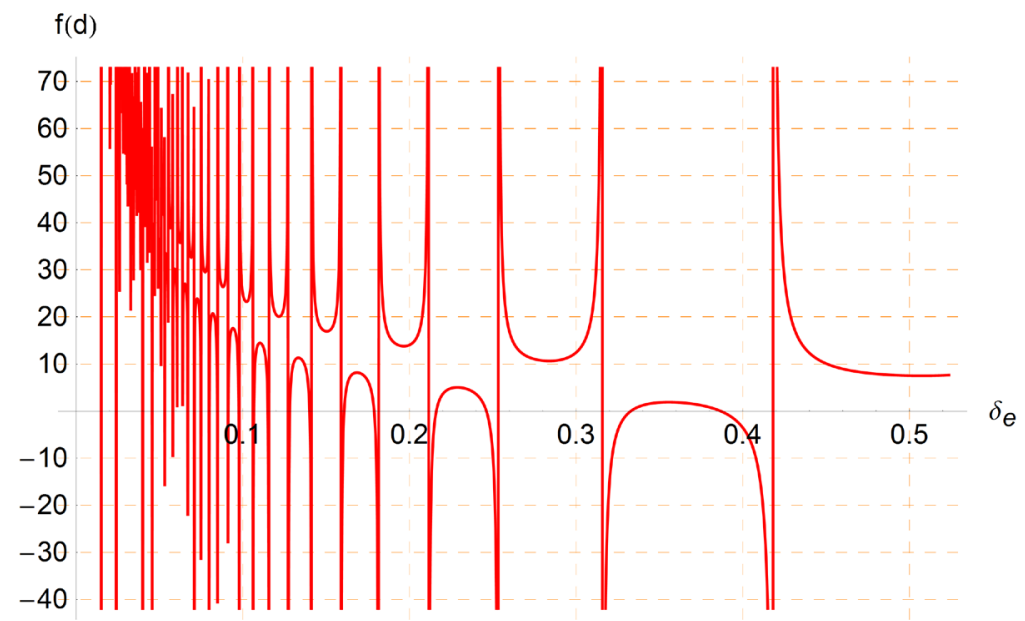

Fig. 9.17. $f(d)$ vs. $\delta_{e}$ ranging from 0 to $\pi / 6\left(d / D_{p}=2\right)$.

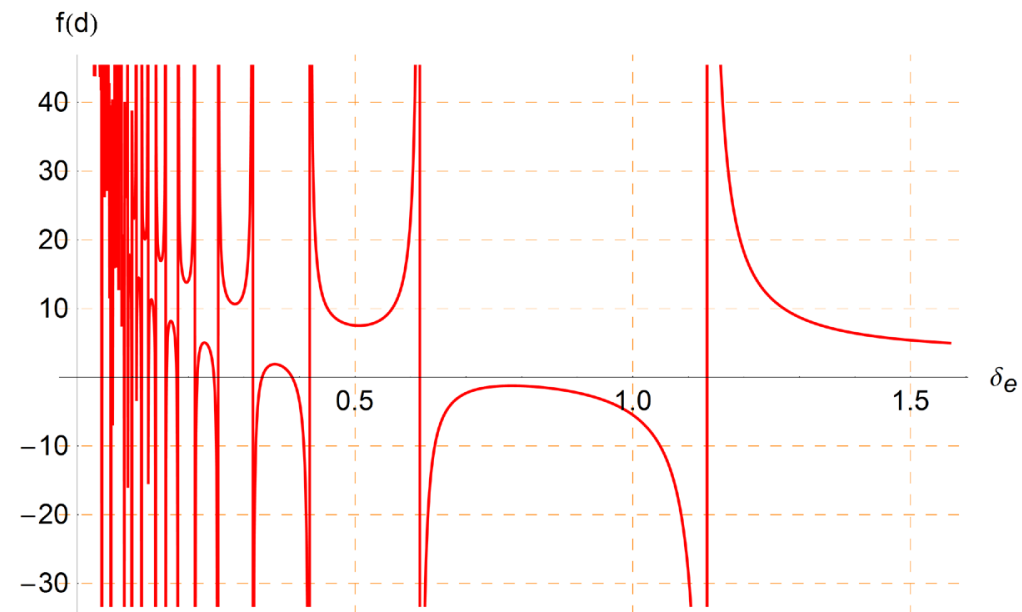

Fig. 9.18. $f(d)$ vs. $\delta_{e}$ ranging from 0 to $\pi / 2\left(d / D_{p}=2\right)$. 


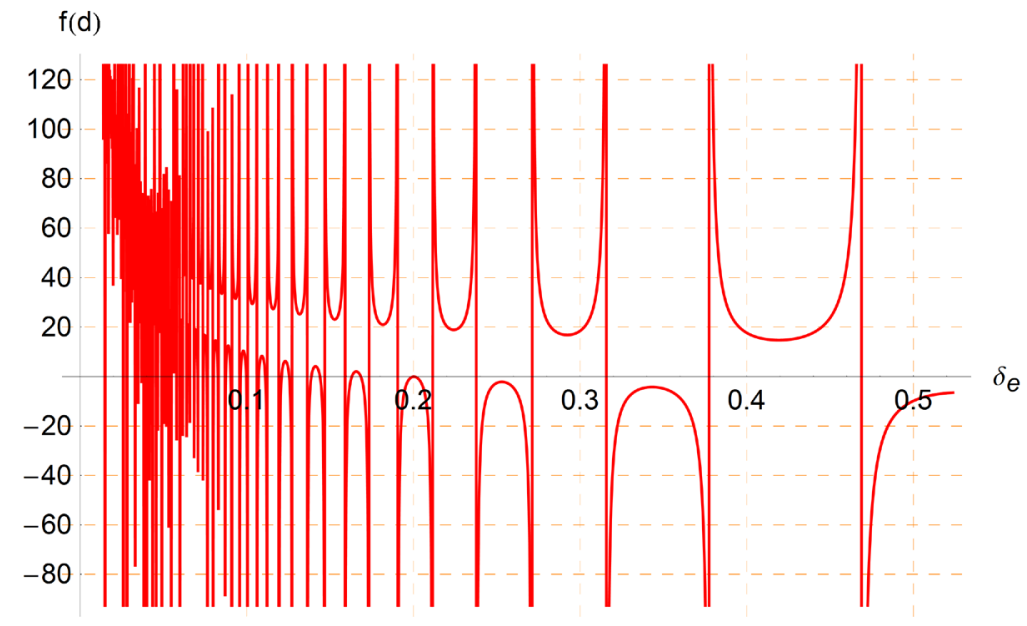

Fig. 9.19. $\mathrm{f}(\mathrm{d})$ vs. $\delta_{e}$ ranging from 0 to $\pi / 6\left(d / D_{p}=3\right)$.

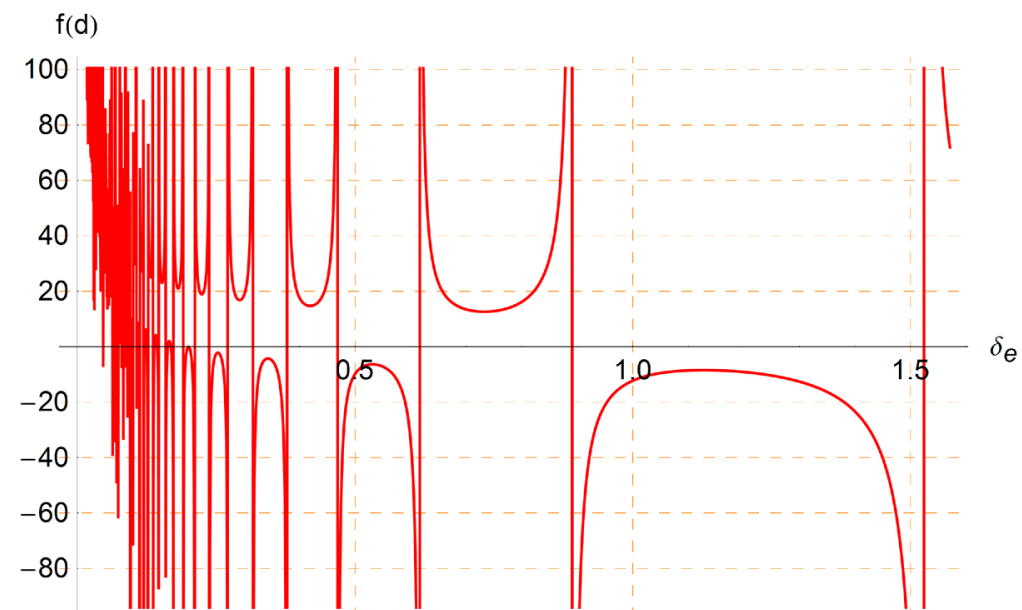

Fig. 9.20. $f(d)$ vs. $\delta_{e}$ ranging from 0 to $\pi / 2\left(d / D_{p}=3\right)$. 


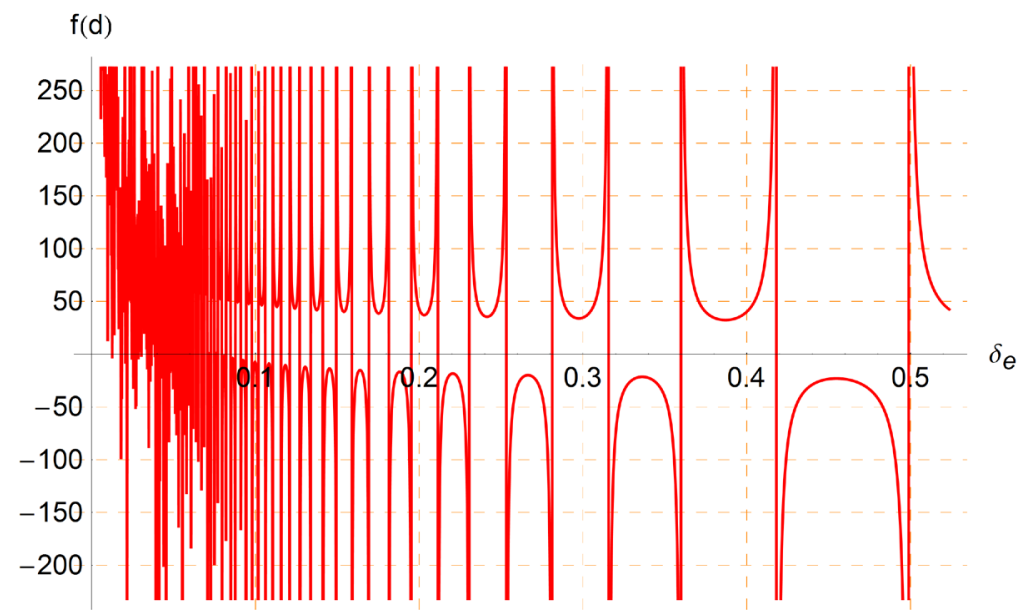

Fig. 9.21. $f(d)$ vs. $\delta_{e}$ ranging from 0 to $\pi / 6\left(d / D_{p}=4\right)$.

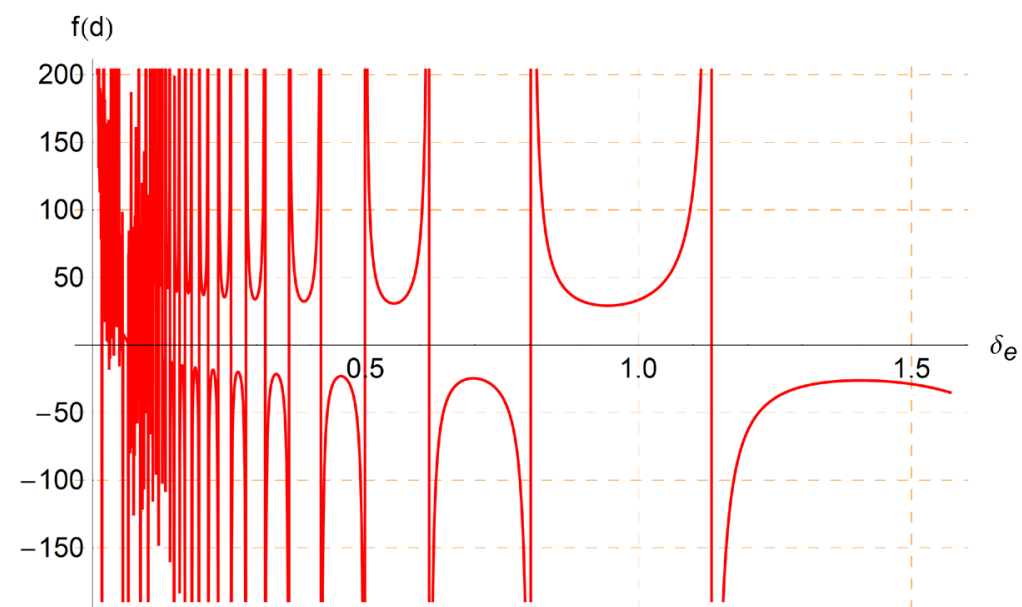

Fig. 9.22. $f(d)$ vs. $\delta_{e}$ ranging from 0 to $\pi / 2\left(d / D_{p}=4\right)$. 


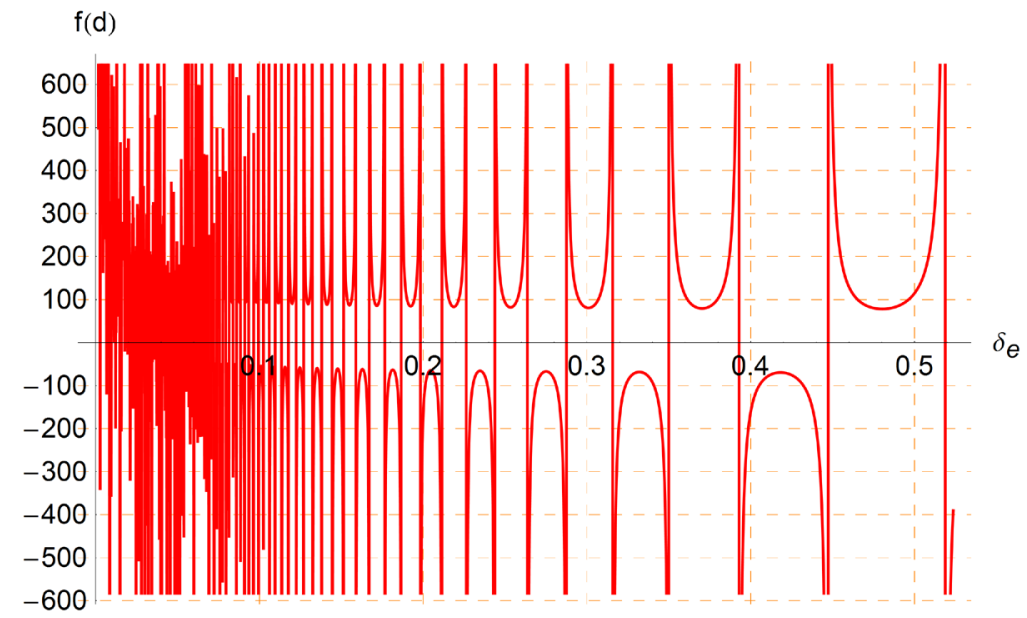

Fig. 9.23. $f(d)$ vs. $\delta_{e}$ ranging from 0 to $\pi / 6\left(d / D_{p}=5\right)$.

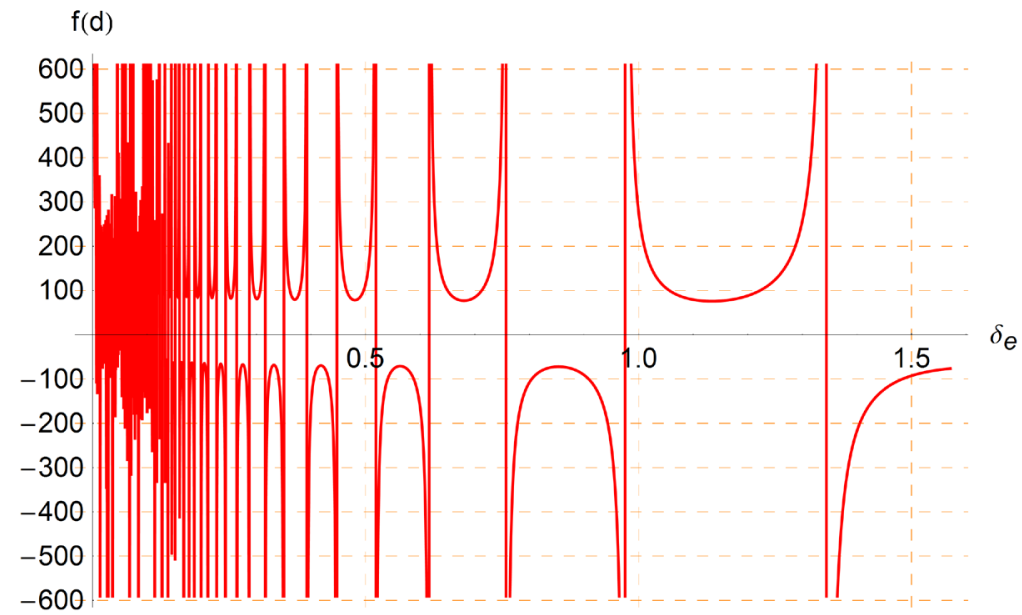

Fig. 9.24. $f(d)$ vs. $\delta_{e}$ ranging from 0 to $\pi / 2\left(d / D_{p}=5\right)$. 


\subsubsection{Thickness for Perfect Impedance Matching of Hematite}

As stated before, hematite shows weak microwave absorption at low temperatures due to its small permittivity and permeability. To attain higher microwave absorption in the whole temperature range, the microwave parameters of hematite measured in this work may be used to show the effectiveness of the function for the evaluation of impedance matching. For hematite, as indicated in eq. (9.23), the value of function is determined by the values of $d / D_{p}$ and $\delta_{e}$. These parameters of hematite can be determined by using the corresponding equations derived in the previous chapters. The results are summarized in Table 9.1.

Table 9.1

Maximum thickness of hematite for perfect impedance matching.

\begin{tabular}{ccccccc}
\hline \multirow{2}{*}{ Temperature $\left({ }^{\circ} \mathrm{C}\right)$} & \multicolumn{2}{c}{$\delta_{e}(\mathrm{rad})$} & \multicolumn{2}{c}{$d / D_{p}$ (Maximum) } & \multicolumn{2}{c}{$d$ (Maximum, m) } \\
\cline { 2 - 7 } & $915 \mathrm{MHz}$ & $2450 \mathrm{MHz}$ & $915 \mathrm{MHz}$ & $2450 \mathrm{MHz}$ & $915 \mathrm{MHz}$ & $2450 \mathrm{MHz}$ \\
\hline 24 & 0.00193 & 0.00166 & 7.63 & 7.78 & 76.68 & 33.95 \\
296 & 0.00496 & 0.0033 & 6.68 & 7.09 & 25.70 & 15.33 \\
591 & 0.3961 & 0.1874 & 2.27 & 2.86 & 0.097 & 0.10 \\
827 & 0.5712 & 0.7663 & 1.60 & - & 0.024 & - \\
982 & 0.2380 & 0.5116 & 2.82 & 1.50 & 0.088 & 0.0089 \\
\hline
\end{tabular}

As shown in Table 9.1, hematite possesses a very low $\delta_{e}$ at $24{ }^{\circ} \mathrm{C}(915 \mathrm{MHz})$. Due to numerous solutions of the derived function at this low $\delta_{e}$, hematite of different thicknesses can be heated without reflection loss. The maximum thickness of hematite for perfect impedance matching can be as high as $\sim 76.68 \mathrm{~m}$ at $24{ }^{\circ} \mathrm{C}$. However, it should be emphasized that although perfect impedance matching can be achievable for hematite at low temperatures, microwave heating of the material could be slow. This is 
because heat generation in materials depends on the amplitude of dielectric loss factor (the imaginary part of permittivity), which is relatively small for hematite at low temperatures according to the dielectric measurement. This suggests that not only a suitable sample size is required but also optimum microwave parameters of the material are necessary to obtain high microwave absorption and thus efficient microwave heating.

As the temperature increases, the maximum thickness of hematite for perfect impedance matching decreases rapidly until $827{ }^{\circ} \mathrm{C}$, resulting from the increased microwave dielectric properties (indicated by $\delta_{e}$ ). Table 9.1 shows that hematite presents the maximum $\delta_{e}$ value $(0.5712)$ at $827{ }^{\circ} \mathrm{C}$. The $\delta_{e}$ value then declines to 0.2380 as the temperature increases to $982{ }^{\circ} \mathrm{C}$. The presence of the maximum loss tangent phase angle is actually due to the formation of the dielectric loss peak of hematite. The maximum thicknesses of hematite for perfect impedance matching are found to be 0.024 and 0.088 m at 827 and $982{ }^{\circ} \mathrm{C}$, respectively. It is obvious that the highest microwave absorption can only be obtained as the sample has the thickness of several centimeters at elevated temperatures. Owing to the high flexibility of thickness selection at low temperatures, the optimal dimension of hematite can be limited to the range below $0.10 \mathrm{~m}$, which is able to avoid a clear temperature nonuniformity throughout the microwave heating process. The impedance matching images for hematite at $915 \mathrm{MHz}$ are shown in Figs. $9.25-9.29$. 


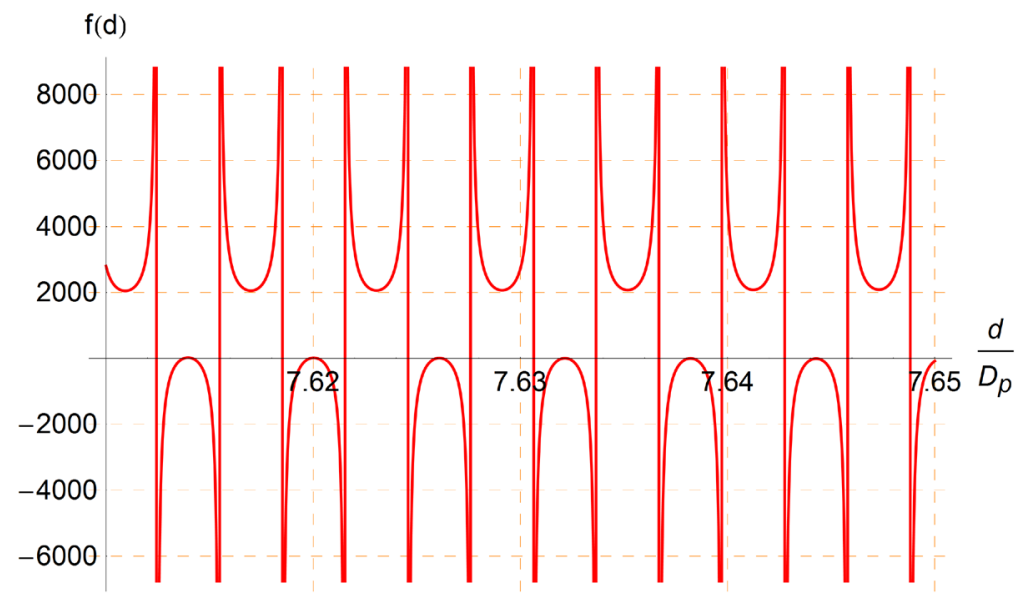

Fig. 9.25. $f(d)$ vs. $d / D_{p}$ ranging from 7.61 to $7.65\left(\delta_{e}=0.00193\right)$.

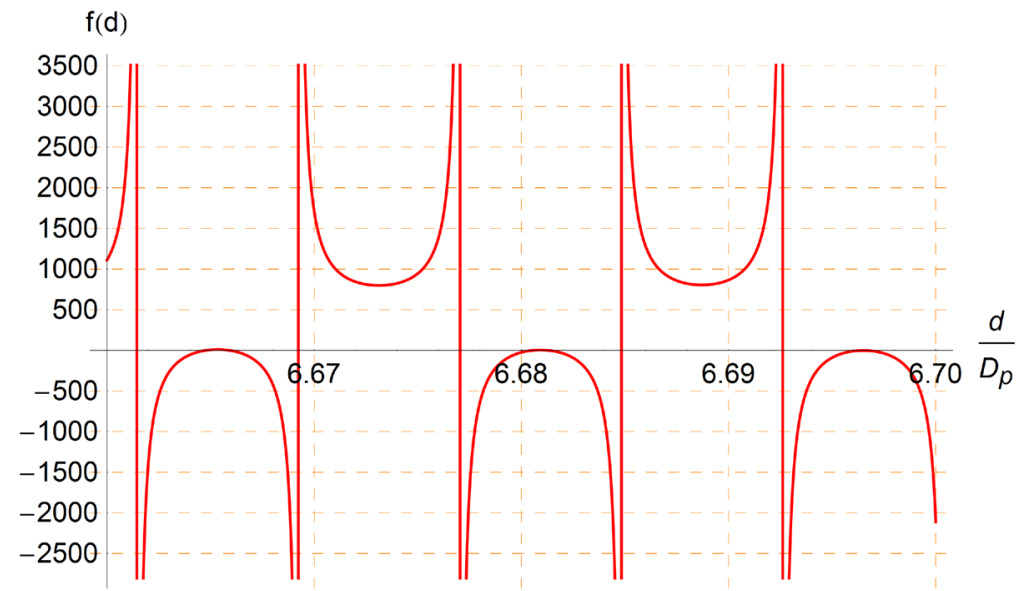

Fig. 9.26. $f(d)$ vs. $d / D_{p}$ ranging from 6.66 to $6.70\left(\delta_{e}=0.00496\right)$. 


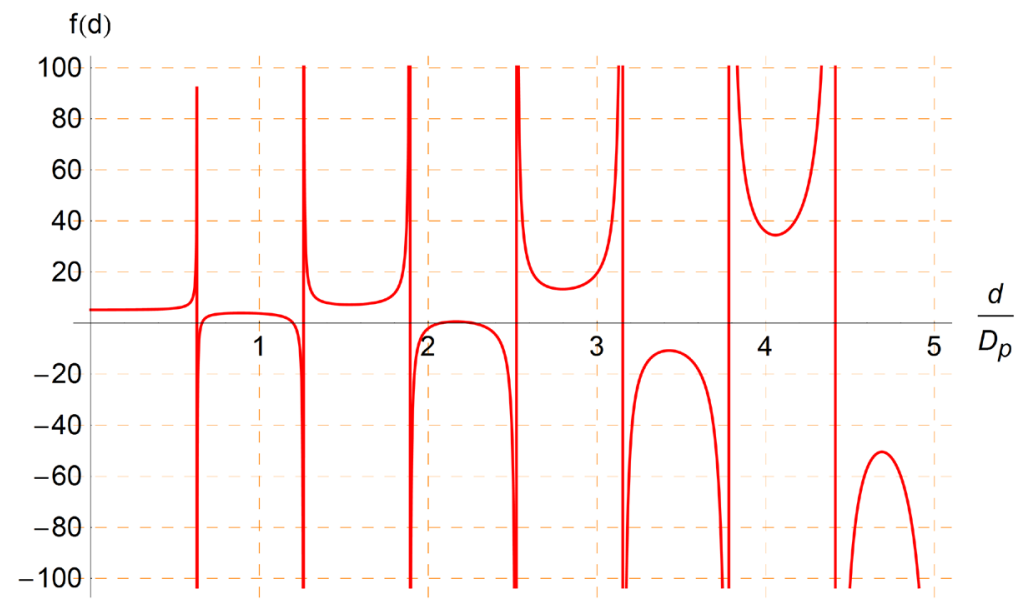

Fig. 9.27. $f(d)$ vs. $d / D_{p}$ ranging from 0 to $5\left(\delta_{e}=0.3961\right)$.

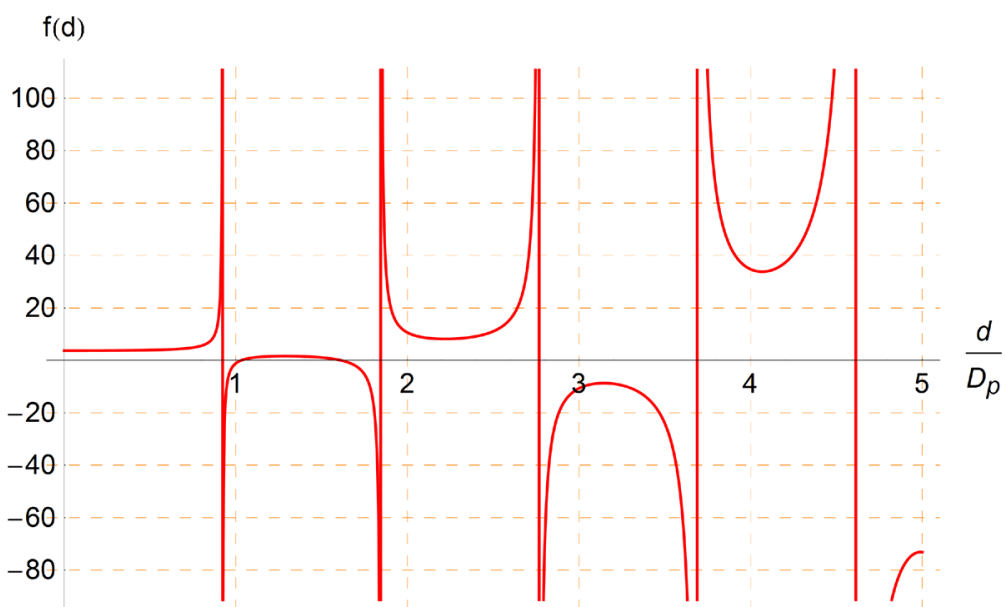

Fig. 9.28. $f(d)$ vs. $d / D_{p}$ ranging from 0 to $5\left(\delta_{e}=0.5712\right)$. 


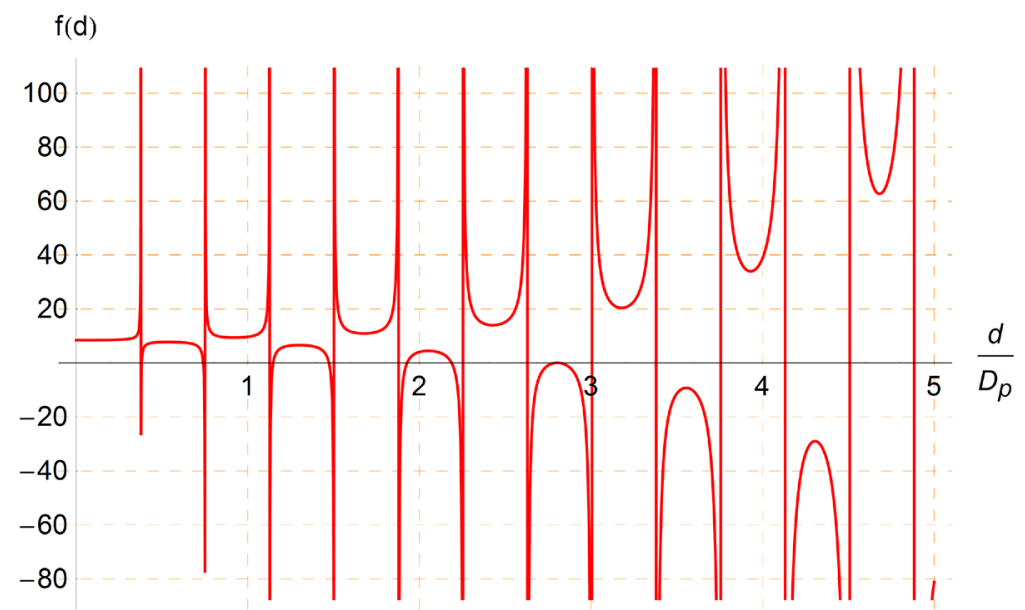

Fig. 9.29. $f(d)$ vs. $d / D_{p}$ ranging from 0 to $5\left(\delta_{e}=0.2380\right)$.

Table 9.1 also presents the maximum thickness of hematite for perfect impedance matching at $2450 \mathrm{MHz}$. It is found that the variation of the thickness at $2450 \mathrm{MHz}$ is similar to that at $915 \mathrm{MHz}$. The only noticeable difference is caused by the high $\delta_{e}$ value (0.7663) of hematite at $827^{\circ} \mathrm{C}$. It is revealed that there is no solution of the impedance matching function. This means that perfect impedance matching does not exist at this temperature. High microwave absorption of hematite without reflection loss is thus impossible under this condition. The impedance matching images for hematite at 2450 $\mathrm{MHz}$ are shown in Figs. 9.30-9.34. 


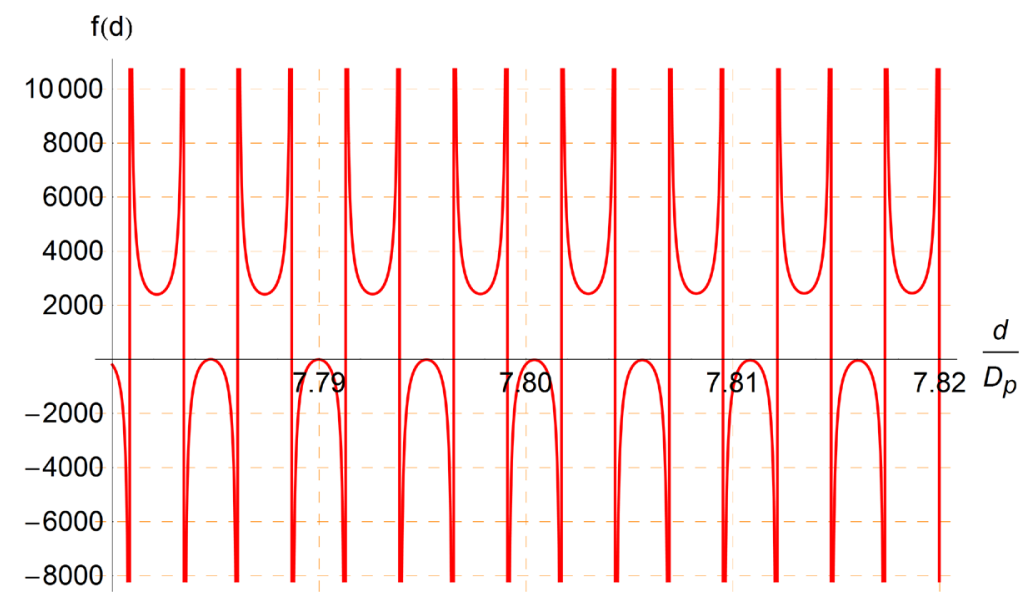

Fig. 9.30. $f(d)$ vs. $d / D_{p}$ ranging from 7.78 to $7.82\left(\delta_{e}=0.00166\right)$.

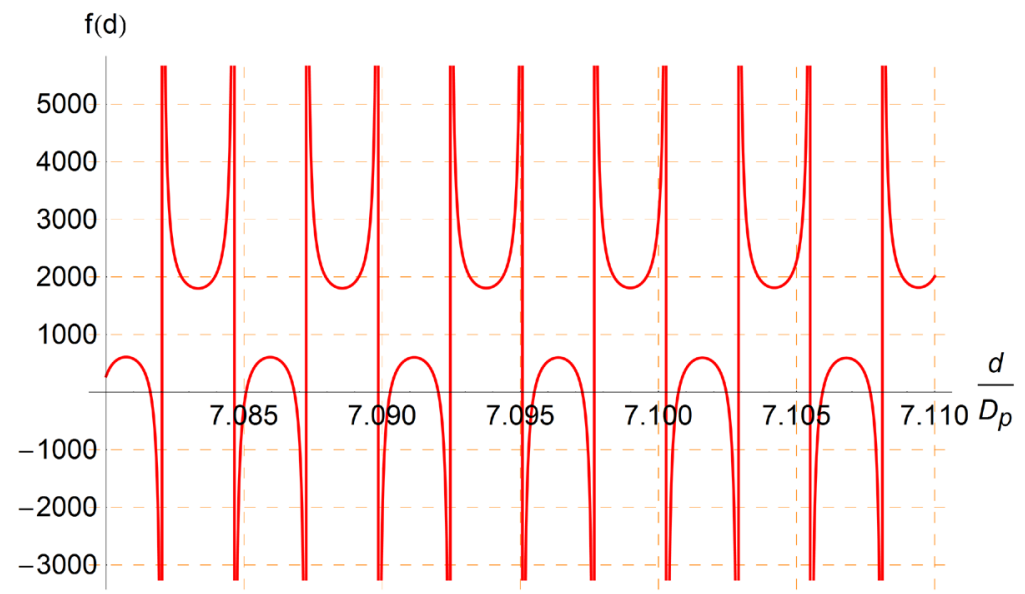

Fig. 9.31. $f(d)$ vs. $d / D_{p}$ ranging from 7.08 to $7.11\left(\delta_{e}=0.0033\right)$. 


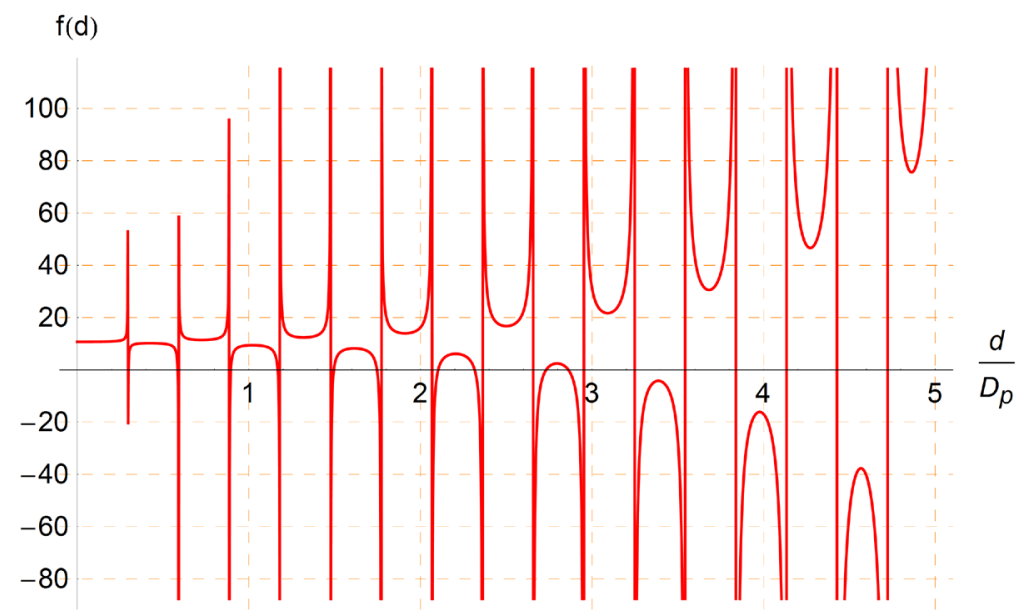

Fig. 9.32. $f(d)$ vs. $d / D_{p}$ ranging from 0 to $5\left(\delta_{e}=0.1874\right)$.

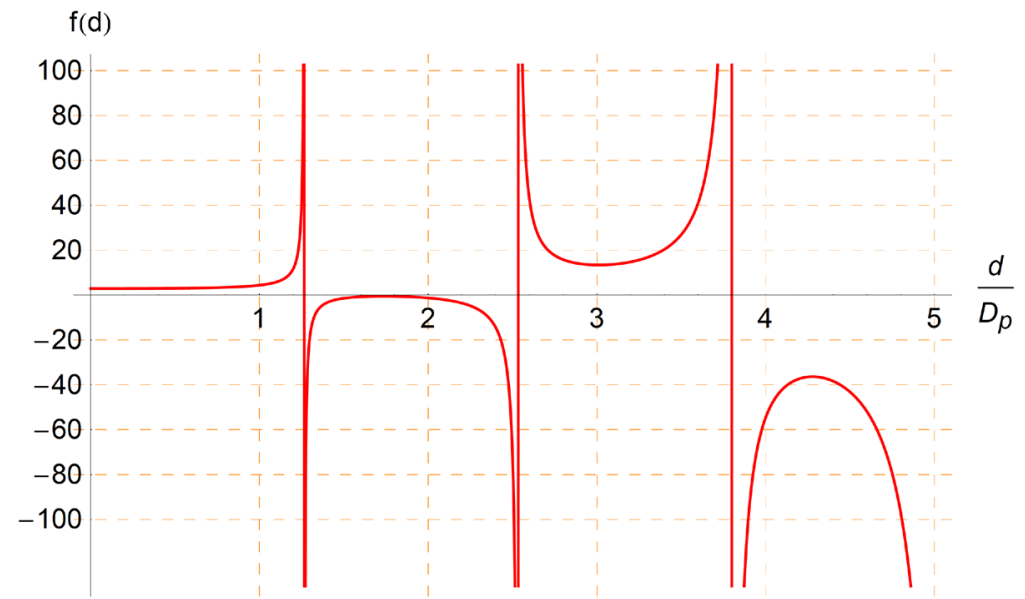

Fig. 9.33. $f(d)$ vs. $d / D_{p}$ ranging from 0 to $5\left(\delta_{e}=0.7663\right)$. 


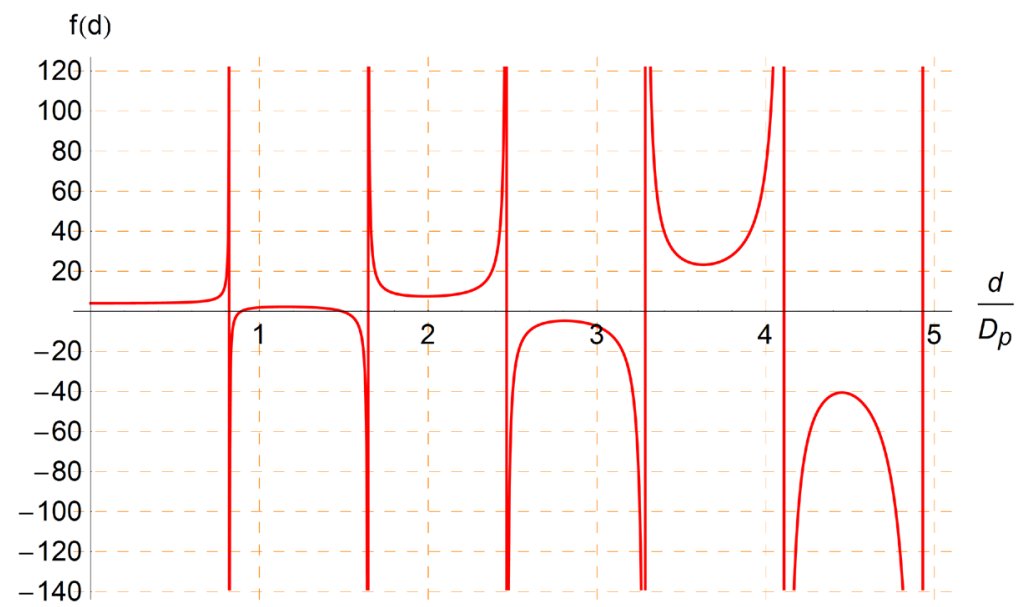

Fig. 9.34. $f(d)$ vs. $d / D_{p}$ ranging from 0 to $5\left(\delta_{e}=0.5116\right)$.

In summary, to increase microwave absorption in microwave heating, a function for evaluating impedance matching degree of absorbers was proposed. It is shown that the value of the function depends on the ratio of the sample thickness $(d)$ to the microwave penetration depth $\left(D_{p}\right)$ and the loss tangent phase angle $\left(\delta_{e}\right)$ for nonmagnetic dielectrics. As the function approaches 0 , perfect impedance matching is achieved, indicating the highest microwave absorption. The maximum sample thickness that corresponds to perfect impedance matching decreases with increasing $\delta_{e}$. It is therefore difficult to attain perfect impedance matching for absorbers with large $\delta_{e}$ values. The calculations of impedance matching for hematite indicate that the optimal dimension of the hematite layer should be less than $0.10 \mathrm{~m}$ to achieve the maximum microwave absorption in the entire microwave heating process. A reasonable dimension of the sample and optimum microwave parameters are quite necessary to obtain perfect impedance matching, and therefore, the highest microwave absorption, which, in turn, will increase the 
microwave heating efficiency. The function developed in this study can be quite helpful in investigating the optimum absorber in microwave heating. 


\section{Chapter 10 Conclusions}

In this study, simplified equations for determining the transverse electromagnetic mode power penetration depth, field attenuation length, and half-power depth of microwaves in materials having both magnetic and dielectric responses were derived from the timedependent Maxwell's curl equations for accurate characterization of the microwave dissipation behaviors in magnetic dielectric materials.

The microwave power absorption formula was also developed from the Poynting vector. A simplified equation for quantifying magnetic loss in materials under microwave irradiation was derived to demonstrate the importance of magnetic loss in microwave heating. The magnetic losses for five ferrites, namely, $\mathrm{BaFe}_{12} \mathrm{O}_{19}, \mathrm{SrFe}_{12} \mathrm{O}_{19}$, $\mathrm{CuFe}_{2} \mathrm{O}_{4}, \mathrm{CuZnFe}_{4} \mathrm{O}_{4}$ and $\mathrm{NiZnFe}_{4} \mathrm{O}_{4}$ were calculated at $2450 \mathrm{MHz}$ using the derived equation. It is found that magnetic loss is up to approximately four times greater than dielectric loss in microwave heating of ferrites. These results, through the calculations, theoretically demonstrate that magnetic dielectric materials are heated much faster in a magnetic field than in an electric field of the microwave applicator.

Numerical (FDTD) modeling and simulations of microwave propagation in various media with varying permittivity and permeability were performed. It is demonstrated that microwave $E$ and $H$ fields do not dissipate in free space while all microwaves are reflected by metal. In the non-lossy dielectric medium, the magnitude of $E$ field 
decreases due to the real part of permittivity. As for microwave lossy materials, although microwave fields decay in both lossy dielectric medium and magnetic dielectric, the fields reduce much faster in the latter owing to the contribution of magnetic response under microwave irradiation. It is also identified that microwave decay in magnetic dielectrics not only relates to the imaginary parts of permittivity and permeability but also associates with the real parts of permittivity and permeability. This observation may be useful in explaining non-thermal microwave effects in microwave chemistry because the traditional viewpoint on heat generation in microwave heating does not consider the effect of real parts of permittivity and permeability.

Microwave absorption properties of typical materials for ironmaking, namely, hematite, magnetite concentrate, ferrous oxide, and coal were characterized using CPMT with varying auxiliary techniques including XRD, FE-SEM, FTIR, TGA, etc. It is shown that there are strong temperature and frequency dependences of permittivity and permeability of the materials.

The $\varepsilon_{r}{ }^{\prime}$ and $\varepsilon_{r}{ }^{\prime \prime}$ values of hematite slightly increase with temperature below $450{ }^{\circ} \mathrm{C}$. Continuous heating to higher temperatures leads to a rapid increase in the $\varepsilon_{r}{ }^{\prime}$ value and a broad dielectric loss peak between 450 and $1000{ }^{\circ} \mathrm{C}$ formed by the $\varepsilon_{r}{ }^{\prime \prime}$ values. The increase in permittivity at high temperatures is closely associated with the sintering effect and the variation of electrical conductivity. The complex relative permeability is 
found to be relatively independent of microwave frequency and temperature below 700 ${ }^{\circ} \mathrm{C}$. The $\mu_{r}{ }^{\prime}$ and $\mu_{r}{ }^{\prime \prime}$ values remain relatively invariable ( 1 and 0 , respectively) in this temperature range, which are consistent with the fact that $\mathrm{Fe}_{2} \mathrm{O}_{3}$ becomes weakly ferromagnetic above the Morin transition at $-13{ }^{\circ} \mathrm{C}$ and below its Néel temperature at $675^{\circ} \mathrm{C}$. As temperature continues to rise, the $\mu_{r}{ }^{\prime}$ values present a decreasing tendency due to the increased electrical conductivity at higher temperatures. The $\mu_{r}{ }^{\prime \prime}$ values, however, stay negligible due to the paramagnetism presented by $\mathrm{Fe}_{2} \mathrm{O}_{3}$ above its Néel temperature. The significant difference between the variation behaviors of permittivity and permeability of $\mathrm{Fe}_{2} \mathrm{O}_{3}$ indicates that the dielectric loss is the primary factor contributing to the microwave absorption of $\mathrm{Fe}_{2} \mathrm{O}_{3}$ and the contribution of magnetic loss to microwave heating can be ignored. The decrease in microwave penetration depth of $\mathrm{Fe}_{2} \mathrm{O}_{3}$ with increasing temperature suggests that $\mathrm{Fe}_{2} \mathrm{O}_{3}$ undergoes a transition from a microwave transparent material to a good microwave absorber as temperature increases.

The experimental data of relative permittivity and permeability of magnetite concentrate (mainly $\mathrm{Fe}_{3} \mathrm{O}_{4}$ ) were presented and used to calculate the power penetration depth as a function of temperature at 915 and $2450 \mathrm{MHz}$. Below $600{ }^{\circ} \mathrm{C}$, the penetration depths determined by eq. (3.22) are much smaller than those calculated by eq. (1.5) as expected when the magnetic losses are included. Above $600{ }^{\circ} \mathrm{C}$, the difference between penetration depths determined by those two equations becomes negligible, reflecting the fact that the magnetic absorption is very small (in principle, zero) above the Curie point of magnetite $\left(585{ }^{\circ} \mathrm{C}\right)$. This shows that the permeability should be considered in the 
determination of penetration depth of magnetic dielectrics, especially at temperatures below the Curie point. A comparison between the calculated microwave dielectric loss and magnetic loss also demonstrates that the magnetic loss plays a major role in the heating of the material. The magnetic loss is important for microwave heating of magnetic dielectrics.

The temperature dependence of the microwave absorption of conventionally heated wüstite (nonstoichiometric ferrous oxide, $\mathrm{Fe}_{0.925} \mathrm{O}$ ) was characterized via the cavity perturbation technique between room temperature and $1100{ }^{\circ} \mathrm{C}$. The complex relative permittivity and permeability of the heated $\mathrm{Fe}_{0.925} \mathrm{O}$ sample slightly change with temperature from room temperature to $200{ }^{\circ} \mathrm{C}$. The dramatic variations of permittivity and permeability of the sample from 200 to $550{ }^{\circ} \mathrm{C}$ are partially attributed to the formation of magnetite $\left(\mathrm{Fe}_{3} \mathrm{O}_{4}\right)$ and metal iron $(\mathrm{Fe})$ from the thermal decomposition of $\mathrm{Fe}_{0.925} \mathrm{O}$, as confirmed by the high temperature X-ray diffraction (HT-XRD). At higher temperatures up to $1100{ }^{\circ} \mathrm{C}$, it is found that $\mathrm{Fe}_{0.925} \mathrm{O}$ regenerates and remains as a stable phase with high permittivity. Because the permittivity dominates the microwave absorption of $\mathrm{Fe}_{0.925} \mathrm{O}$ above $550{ }^{\circ} \mathrm{C}$ resulting in shallow microwave penetration depths ( $\sim 0.11 \mathrm{~m}$ and $\sim 0.015 \mathrm{~m}$ at $915 \mathrm{MHz}$ and $2450 \mathrm{MHz}$, respectively), the regenerated nonstoichiometric ferrous oxide exhibits the useful microwave absorption capability at high temperatures $\left(550-1100{ }^{\circ} \mathrm{C}\right)$. Investigation of thermal stability of the reactions shows that the decomposition proceeds under kinetic reaction control while the regeneration is under thermodynamic control. Kinetics study based on the Coats- 
Redfern integral approximation method indicates that the decomposition reaction follows a Komatsu-Uemura model-based diffusion mechanism with activation energy of $32.860 \mathrm{~kJ} \mathrm{~mol}^{-1}$.

The dielectric properties of an eastern high volatile bituminous coal from West Virginia were measured at 915 and $2450 \mathrm{MHz}$ during pyrolysis from room temperature to 900 ${ }^{\circ} \mathrm{C}$. The dielectric properties remain relatively stable at low temperatures. Apparent increases in dielectric constant and loss factor of the coal were observed in the temperature range of $500-750{ }^{\circ} \mathrm{C}$ due to the release of volatiles. The devolatilization of the coal during pyrolysis, resulting from the losses of carbonyl, aromatic $\mathrm{C}=\mathrm{C}$, vinylic $\mathrm{C}=\mathrm{C}$ and other O-containing functional groups, leads to the increased conductivity. The calculation of microwave penetration depth confirms that the pyrolysis process significantly improves the microwave absorption capability of the coal above $750{ }^{\circ} \mathrm{C}$.

Numerical simulations of heat transfer during the microwave heating process of magnetite (1-D/2-D) subjected to heat conduction, convection, and radiation were performed. The temperature in the object increases rapidly in one minute and a nonuniform temperature distribution inside the object is observed. An obvious temperature hot spot is formed in the corner of the 2-D object initially, which migrates inward with a slower speed with increasing heating time. Continuous heating after $60 \mathrm{~s}$ did not contribute too much to the increase in the temperature of the hot spot, which remains around $1100{ }^{\circ} \mathrm{C}$. A higher power input gives rise to a larger temperature 
increase at the same heating time and a shift of hot spot towards the center of the object is observed. Microwave heating at $915 \mathrm{MHz}$ exhibits better heating uniformity than $2450 \mathrm{MHz}$ mainly because of a longer penetration depth. Heating homogeneity in the object is improved by reducing the dimension $(L)$ of the object from 0.2 to $0.05 \mathrm{~m}$. It is proved that factors including heating time, microwave power, microwave frequency and object dimension should be considered to obtain high heating performance and to avoid/minimize thermal runaway resulting from temperature nonuniformity in largescale microwave processing of magnetic dielectrics.

The effect of absorber dimension on microwave heating was discussed in this study. It shows that, to achieve high microwave heating efficiency, an optimal dimension needs to be determined via the analyses of microwave absorption and heat transfer in materials. Based on the characterization of microwave absorption properties, the calculated reflection loss over the temperature range of heating was found to be useful for obtaining a rapid optimization of absorber dimension, which increases microwave absorption and achieves relatively uniform heating.

To further improve the heating effectiveness, a function for evaluating impedance matching degree of absorbers in microwave heating was proposed. The relation between the impedance matching function $f(d)$ and the variables $d / D_{p}$ and $\delta_{e}$ was established. It can be predicted that there are possible solutions of the function $f(d)$, depending on the values of $d / D_{p}$ and $\delta_{e}$. The perfect matching thickness is found to increase with 
decreasing loss tangent phase angle. It is therefore difficult to approach perfect impedance matching for absorbers with too large loss tangent phase angles. The results indicate that a reasonable dimension of the sample and optimum microwave parameters are necessary to obtain perfect impedance matching, and therefore, the highest microwave absorption, which, in turn, will increase the microwave heating efficiency. 


\section{References}

1. Das S, Mukhopadhyay AK, Datta S, Basu D. Prospects of microwave processing: An overview. Bulletin of Materials Science. 2009;32(1):1-13.

2. Adam D. Microwave chemistry - out of the kitchen. Nature. 2003;421(6923): 571-572.

3. Roy R, Komameni S, Yang JL. Controlled microwave heating and melting of gels. Journal of the American Ceramics Society. 1985;68(7):392-395.

4. Komameni S, Roy R. Anomalous microwave melting of zeolites. Materials Letters, 1986;4(2):107-110.

5. Yang Q, Zhang H, Liu Y, Wen Q, Jia J. The magnetic and dielectric properties of microwave sintered yttrium iron garnet (YIG). Materials Letters. 2008;62(17-18):2647-2650.

6. Sutton WH. Microwave processing of ceramic materials. American Ceramic Society Bulletin. 1989;68(2):376-386.

7. Segerer H. Producing technical ceramics by microwave drying. American Ceramic Society Bulletin. 1998;77(3):64-66.

8. Seyrankaya A, Ozalp B. Dehydration of sodium carbonate monohydrate with indirect microwave heating. Thermochimica Acta. 2006;448(1):31-36.

9. Leonelli C, Pellacani GC, Siligardi C, Veronesi P. Microwave assisted burn-out of organic compounds in ceramic systems. Key Engineering Materials. 2004; 264-268:739-742.

10. Goldstein A, Giefman L, Bar Ziv S. Susceptor assisted microwave sintering of $\mathrm{MgAl}_{2} \mathrm{O}_{4}$ powder at $2.45 \mathrm{GHz}$. Journal of Materials Science Letters. 1998; 17(12):977-979.

11. Goldstein A, Travitzky N, Singurindy A, Kravchik M. Direct microwave sintering of yttria-stabilized zirconia at $2.45 \mathrm{GHz}$. Journal of the European Ceramic Society. 1999;19(12):2067-2072.

12. Lee KY, Case ED. Microwave sintering of alumina matrix zirconia composites using a single-mode microwave cavity. Journal of Materials Science Letters. 1999;18(3):201-203. 
13. Xie Z, Yang J, Huang X, Huang Y. Microwave processing and properties of ceramics with different dielectric loss. Journal of the European Ceramic Society. 1999;19(3):381-387.

14. Fang Y, Agrawal DK, Roy DM, Roy R. Fabrication of porous hydroxyapatite ceramics by microwave processing. Journal of Materials Research. 1992;7(2): 490-494.

15. Fang Y, Agrawal DK, Roy DM, Roy R. J. Microwave sintering of hydroxyapatite. Journal of Materials Research. 1994;9(1):180-187.

16. Fang Y, Agrawal DK, Roy DM, Roy R. Fabrication of transparent hydroxyapatite ceramics by microwave processing. Materials Letters. 1995; 23(1):147-151.

17. Breval E, Cheng JP, Agrawal DK, Gigl P, Dennis M, Roy R, Papworth AJ. Comparison between microwave and conventional sintering of $\mathrm{WC} / \mathrm{Co}$ composites. Materials Science and Engineering A. 2005;391(1-2):285-295.

18. Tun KS, Gupta M. Improving mechanical properties of magnesium using nanoyttria reinforcement and microwave assisted powder metallurgy method. Composites Science and Technology. 2007;67(13):2657-2664.

19. Hsu CS, Huang CL. Effect of $\mathrm{CuO}$ additive on sintering and microwave dielectric behavior of $\mathrm{LaAlO}_{3}$ ceramics. Materials Research Bulletin. 2001;36 (11):1939-1947.

20. Huang $\mathrm{CL}$, Weng $\mathrm{MH}$. The effect of $\mathrm{PbO}$ loss on microwave dielectric properties of $(\mathrm{Pb}, \mathrm{Ca})(\mathrm{Zr}, \mathrm{Ti}) \mathrm{O}_{3}$ ceramics. Materials Research Bulletin. 2001;36(3-4):683-691.

21. Rhee S, Agrawal D, Shrout T, Thumm M. Investigation of high frequency $(2.45$ $\mathrm{GHz}, 30 \mathrm{GHz}$ ) sintering for Pb-based ferroelectrics and microscale functional devices. Ferroelectrics. 2001;261(1):15-21.

22. Agrawal D. Microwave sintering of ceramics, composites and metallic materials, and melting of glasses. Transactions - Indian Ceramic Society. 2006;65(3):129-144.

23. Chen M, Siochi EJ, Ward TC, McGrath JE. Basic ideas of microwave processing of polymers. Polymer Engineering and Science. 1993;33(17):1092-1109.

24. Lee WI, Springer GS. Microwave curing of composites. Journal of Composite Materials.1984;18(4):387-409. 
25. Jordan C, Galy J, Pascault JP. Comparison of microwave and thermal cure of an epoxy/amine matrix. Polymer Engineering and Science. 1995;35(3):233-239.

26. Zhang G, Leparoux S, Liao H, Coddet C. Microwave sintering of poly-etherether-ketone (PEEK) based coatings deposited on metallic substrate. Scripta Materialia. 2006;55(7):621-624.

27. Aravindan S, Krishnamurthy R. Joining of ceramic composites by microwave heating. Materials Letters. 1999;38(4):245-249.

28. Cawley JD. Joining of ceramic matrix composites. American Ceramic Society Bulletin. 1989;68(9):1619-1623.

29. Cheng J, Agrawal D, Roy R. Microwave synthesis of aluminum oxynitride (AlON). Journal of Materials Science Letters. 1999;18(24):1989-1990.

30. Meek TT, Blake RD. Ceramic-ceramic seals by microwave heating. Journal of Materials Science Letters. 1986;5(3):270-274.

31. Zeng L, Case ED, Crimp MA, Effects of a silica spin-on interlayer and heating mode on the joining of zirconia and $\mathrm{MaCor}^{\mathrm{TM}}$. Materials Science and Engineering: A- Structural Materials: Properties, Microstructure and Processing. 2003;357(1-2):67-74.

32. Fukushima H, Yamanaka T, Matsui M. Microwave heating of ceramics and its application to joining. Journal of Materials Research. 1990;5(2):397-405.

33. Chandrasekaran S, Basak T, Ramanathan S. Experimental and theoretical investigation on microwave melting of metals. Journal of Materials Processing Technology. 2011;211(3):482-487.

34. Duval DJ, Phillips BL, Terjak MJE, Risbud SH. Reversible color changes and structural implications of microwave melting ion-conducting glasses. Journal of Solid State Chemistry. 1997;131(1):173-176.

35. Berteaud AJ, Badot JC. High temperature heating in refractory materials. Journal of Microwave Power. 1976;11(4):315-320.

36. Apte SK, Naik SD, Sonawane RS, Kale BB, Pavaskar N, Mandale AB, Das BK. Nanosize $\mathrm{Mn}_{3} \mathrm{O}_{4}$ (Hausmannite) by microwave irradiation method. Materials Research Bulletin. 2006;41(3):647-654.

37. Rao KJ, Vaidhyanathan B, Ganguli M, Ramakrishnan PA: synthesis of inorganic solids using microwaves. Chemistry of Materials. 1999;11(4):882-895. 
38. Rao KJ, Mahesh K, Kumar S. A strategic approach for preparation of oxide nanomaterials. Bulletin of Materials Science. 2005;28(1):19-24.

39. Watawe SC, Keluskar S, Gonbare, Tangsali R: Preparation and magnetic properties of cadmium substituted lithium ferrite using microwave-induced combustion. Thin Solid Films. 2006;505(1-2):168-172.

40. Lagashetty A, Havanoor V, Basavaraja S, Balaji SD, Venkataraman A. Microwave-assisted route for synthesis of nanosized metal oxides. Science and Technology of Advanced Materials. 2007;8(6):484-493.

41. Siddiqueya IA, Furusawaa T, Satoa M, Bahadurc NM, Suzuki N. Fabrication of silica coated $\mathrm{Al}_{2} \mathrm{O}_{3}$ nanoparticles via a fast and facile route utilizing microwave irradiation. Materials Chemistry and Physics. 2011;130(1-2):583-586.

42. Bykov YV, Rybakov KI, Semenov VE. High-temperature microwave processing of materials. Journal of Physics D: Applied Physics. 2001;34(13):R55-R75.

43. Standish N, Worner H. Gupta G. Temperature distribution in microwave-heated iron ore-carbon composites. Journal of Microwave Power and Electromagnetic Energy. 1990;25(2):75-80.

44. Standish N, Worner H. Microwave application in the reduction of metal oxides with carbon. Journal of Microwave Power and Electromagnetic Energy. 1990; 25(3):177-180.

45. Standish N, Pramusanto. Reduction of microwave irradiated iron ore particles in CO. ISIJ International. 1991;31(1):11-16.

46. Standish N, Huang W. Microwave application in carbothermic reduction of iron ores. ISIJ International. 1991; 31(3):241-245.

47. Ishizaki K, Nagata K, Hayashi T. Production of pig iron from magnetite ore-coal composite pellets by microwave heating. ISIJ International. 2006;46(10):1403-1409.

48. Yoshikawa N, Ishizuka E, Mashiko K, Chen Y, Taniguchi S. Brief review on microwave (MW) heating, Its application to iron \& steel industry and to the relevant environmental techniques. ISIJ International. 2007;47(4):523-527.

49. Malmberg D, Hahlin P, Nilsson E. microwave technology in steel and metal industry, an overview. ISIJ International. 2007;47(4):533-538.

50. Ishizaki K, Nagata K. Selectivity of microwave energy consumption in the 
reduction of $\mathrm{Fe}_{3} \mathrm{O}_{4}$ with carbon black in mixed powder. ISIJ International. 2007; 47(6):811-816.

51. Ishizaki K, Nagata K, Hayashi T. localized heating and reduction of magnetite ore with coal in composite pellets using microwave irradiation. ISIJ International. 2007;47(6):817-822.

52. Ishizaki K, Nagata K. Microwave induced solid-solid reactions between $\mathrm{Fe}_{3} \mathrm{O}_{4}$ and Carbon black powders. ISIJ International. 2008;48(9):1159-1164.

53. Hotta M, Hayashi M, Nishikata A, Nagata K. Complex permittivity and permeability of $\mathrm{SiO}_{2}$ and $\mathrm{Fe}_{3} \mathrm{O}_{4}$ Powders in microwave frequency range between 0.2 and 13.5 GHz. ISIJ International. 2009;49(9):1443-1448.

54. Hotta M, Hayashi M, Nagata K. High temperature measurement of complex permittivity and permeability of $\mathrm{Fe}_{3} \mathrm{O}_{4}$ powders in the frequency range of 0.2 to 13.5 GHz. ISIJ International. 2011;51(3):491-497.

55. Hotta M, Hayashi M, Lanagan MT, Agrawal DK, Nagata K. Complex permittivity of graphite, carbon black and coal powders in the ranges of X-band frequencies $(8.2$ to $12.4 \mathrm{GHz}$ ) and between 1 and $10 \mathrm{GHz}$. ISIJ International. 2011;51(11):1766-1772.

56. Wang Y. Direct Steelmaking Through Microwave and Electric Arc Heating. Ph.D. Dissertation. Michigan Technological University, Houghton, MI, 2005.

57. Huang X. Novel direct steelmaking by combining microwave, electric arc and exothermal heating technologies. Final Technical Report. U.S. Department of Energy. 2005;40-48.

58. Hwang JY, Huang X. New steel production technology with microwave and electric arc heating. 2006 TMS Fall Extraction and Processing Division: Sohn International Symposium, Sohn International Symposium: Advanced Processing of Metals and Materials - Proceedings of the International Symposium. 2006;5: 251-261.

59. Hwang JY, Huang X, Shi S. Steel production with microwave assisted electric arc furnace technology. TMS Annual Meeting, Materials Processing Under the Influence of External Fields- TMS 2007 Annual Meeting and Exhibition. 2007;225-234.

60. Kriegsmann GA. Thermal runaway in microwave heated ceramics: a onedimensional model. Journal of Applied Physics. 1992;71(4):1960-1966. 
61. Vriezinga CA. Thermal runaway in microwave heated isothermal slabs, cylinders, and spheres. Journal of Applied Physics. 1997;83(1):438-442.

62. Vriezinga CA. Thermal profiles and thermal runaway in microwave heated slabs. Journal of Applied Physics. 1999;85(7):3774-3779.

63. Wu X. Thomas, JR, Davis WA. Control of thermal runaway in microwave resonant cavities. Journal of Applied Physics. 2002;92(6):3374-3380.

64. Wu X. Experimental and Theoretical Study of Microwave Heating of Thermal Runaway Materials. Ph.D. Dissertation. Virginia Polytechnic Institute and State University, Blacksburg, VA, 2002.

65. Peng Z, Hwang JY, Mouris J, Hutcheon R, Huang X. Microwave penetration depth in materials with non-zero magnetic susceptibility. ISIJ International. 2010; 50(11):1590-1596.

66. Guo L, Ji G, Chang X, Zheng M, Shi Y, Zheng Y. Microwave-assisted synthesis of Sb2Se3 submicron tetragonal tubular and spherical crystals. Nanotechnology. 2010;21(3):035606-035606-7.

67. Inan US. Inan AS. Electromagnetic Waves. Prentice Hall, Upper Saddle River, NJ, 1999.

68. Hayt WH, Buck HA. Engineering Electromagnetics. McGraw-Hill, New York, NY, 2001.

69. Harahsheh MA, Kingman SW. Microwave-assisted leaching-a review. Hydrometallurgy. 2004;73(3-4):189-203.

70. Mingos DM, Baghurst DR. Tilden Lecture. Applications of microwave dielectric heating effects to synthetic problems in chemistry. Chemical Society Reviews. 1991;20(1):1-47.

71. Thostenson ET, Chou TW. Microwave processing: fundamentals and applications. Composites Part A: Applied Science and Manufacturing. 1999; 30(9):1055-1071.

72. Wu YP, Ong CK, Lin GQ, Li ZW. Improved microwave magnetic and attenuation properties due to the dopant $\mathrm{V}_{2} \mathrm{O}_{5}$ in $\mathrm{W}$-type barium ferrites. Journal of Physics D: Applied Physics. 2006;39(14):2915-2919.

73. Pickles CA. Microwaves in extractive metallurgy: part 1- review of fundamentals. Minerals Engineering. 2009;22(13):1102-1111. 
74. Ford JD, Pei DCT. High temperature chemical processing via microwave absorption. Journal of Microwave Power. 1967;2(2):61-64.

75. Chen TT, Dutrizac JE, Haque KE, Wyslouzil W, Kashyap S. The relative transparency of minerals to microwave radiation. Canadian Metallurgical Quarterly. 1984;23(3):349-351.

76. Hua Y, Lin Z, Yan Z. Application of microwave irradiation to quick leach of zinc silicate ore. Minerals Engineering. 2002;15(6):451-456.

77. Kulumbaev EB, Semenov VE, Rybakov KI. Stability of microwave heating of ceramic materials in a cylindrical cavity. Journal of Physics D: Applied Physics. 2007;40(21):6809-6817.

78. Xia DK, Pickles CA. Applications of microwave energy in extractive metallurgy: A review. CIM Bulletin. 1997;90(1011):96-107.

79. Yang Q, Zhang H, Liu Y, Wen Q, Jia L. The magnetic and dielectric properties of microwave sintered yttrium iron garnet (YIG). Materials Letters. 2008;62(17-18):2647-2650.

80. Mu G, Chen N, Pan X, Shen H, Mu M. Preparation and microwave absorption properties of barium ferrite nanorods. Materials Letters. 2008;62(6-7):840-842.

81. Kang YQ, Cao MS, Yuan J, Shi XL. Microwave absorption properties of multiferroic $\mathrm{BiFeO}_{3}$ nanoparticles. Materials Letters. 2009;63(15):1344-1346.

82. Hwang JY, Huang X, Qu S,Wang Y, Shi S, Caneba G. Iron oxide reduction with conventional and microwave heating under $\mathrm{CO}$ and $\mathrm{H}_{2}$ atmospheres. TMS Annual Meeting, EPD Congress 2006 - Proceedings of Sessions and Symposia sponsored by the Extraction and Processing Division of The Minerals, Metals and Materials Society held during the 2006 TMS Annual Meeting. 2006; 219-227.

83. Hara K, Hayashi M, Sato M, Nagata K. Continuous pig iron making by microwave heating with $12.5 \mathrm{~kW}$ at $2450 \mathrm{GHz}$. Journal of Microwave Power and Electromagnetic Energy. 2011;45(3):137-147.

84. Clark DE, Folz DC, West JK: Processing materials with microwave energy. Materials Science and Engineering A-Structural Materials: Properties, Microstructure and Processing. 2000;287(2):153-158.

85. Metaxas AC, Meredith RJ. Industrial Microwave Heating. Peter Peregrinus, London, 1983. 
86. Cheng J, Roy R, Agrawal D. Experimental proof of major role of magnetic field losses in microwave heating of metal and metallic composites. Journal of Materials Science Letters. 2001;20(17):1561-1563.

87. Cheng J, Agrawal D, Zhang Y, Roy R. Development of translucent aluminum nitride (AIN) using microwave sintering process. Journal of Electroceramics. 2002;9(1):67-71.

88. Cheng J, Roy R, Agrawal D. Radically different effects on materials by separated microwave electric and magnetic fields. Materials Research Innovations. 2002; 5(3-4):170-177.

89. Poynting JH. On the transfer of energy in the electromagnetic field. Philosophical Transactions of the Royal Society of London. 1884;175:343-361.

90. Cirpar C. Heat Treatment of Iron Ore Agglomerates with Microwave Energy. Master's Thesis. Middle East Technical University, Ankara, Turkey. 2005.

91. Stuchly MA, Stuchly SS. Coaxial line reflection methods for measuring dielectric properties of biological substances at radio and microwave frequencies-a review. IEEE Transactions on Instrumentation and Measurement.1980;29(3):176-183.

92. Blackham DV, Pollard RD. An improved technique for permittivity measurements using a coaxial probe. IEEE Transactions on Instrumentation and Measurement.1997;46(5):1093-1099.

93. Otto GP, Chew WC. Improved calibration of a large open- ended coaxial probe for dielectric measurements. IEEE Transactions on Instrumentation and Measurement. 1991;40(4):742-746.

94. Ghodgaonkar DK, Varadan VV, Varadan VK. A free-space method for measurement of dielectric constants and loss tangents at microwave frequencies. IEEE Transactions on Instrumentation and Measurement. 1989;38(3):789-793.

95. Courtney CC. Time-domain measurement of the electromagnetic properties of materials. IEEE Transactions on Microwave Theory and Techniques. 1998; 46(5):517-522.

96. Komarov VV, Yakovlev VV. Modeling control over determination of dielectric properties by perturbation technique. Microwave and Optical Technology Letters. 2003;39(6):443-446.

97. Chen L, Ong CK, Tan BTG. Amendment of cavity perturbation method for permittivity measurement of extremely low-loss dielectrics. IEEE Transactions 
on Instrumentation and Measurement. 1999;48(6):1031-1037.

98. Kraszewski AW, Nelson SO. Observations on resonant cavity perturbation by dielectric objects. IEEE Transactions on Microwave Theory and Techniques. 1992;40(1):151-156.

99. Pickles CA, Mouris J, Hutcheon RM. High-temperature dielectric properties of goethite from 400 to $3000 \mathrm{MHz}$. Journal of Materials Research. 2005;20(1):18-29.

100. Hutcheon RM, de Jong M, Adams F. A system for rapid measurements of RF and microwave properties up to $1400^{\circ} \mathrm{C}$. Part 1: theoretical development of the cavity frequency- shift data analysis equations. Journal of Microwave Power and Electromagnetic Energy. 1992;27(2):87-92.

101. Adams F, de Jong M, Hutcheon RM. Sample shape correction factors for cavity perturbation measurements. Journal of Microwave Power and Electromagnetic Energy. 1992;27(3):131-135.

102. Hutcheon RM, Morin GF, Smith BH, de Jong M, Adams F. The high temperature microwave response of industrial ferrites. Ceramic Transactions. Microwaves: Theory and Application in Materials Processing II. 1993;36:469-481.

103. Ho YC, Yam KL. Effect of metal shielding on microwave heating uniformity of a cylindrical food model. Journal of Food Processing and Preservation. 1992; 16(5):337-359.

104. Vadivambal R, Jayas DS. Non-uniform temperature distribution during microwave heating of food materials-a review. Food and Bioprocess Technology. 2010;3(2):161-171.

105. Pert E, Carmel Y, Birnboim A, Olorunyolemi T, Gershon D, Calame J, Lloyd IK, Wilson OC. Temperature measurements during microwave processing: the significance of thermocouple effects. Journal of the American Ceramic Society. 2001;84(9):1981-1986.

106. Dolande J, Datta A. Temperature profiles in microwave heating of solids: a systematic study. Journal of Microwave Power and Electromagnetic Energy. 1993;28(2):58-67.

107. Fleischman G. Predicting temperature range in food slabs undergoing long term/low power microwave heating. Journal of Food Engineering. 1996;27(4): $337-351$. 
108. Fleischman GJ. Predicting temperature range in food slabs undergoing shortterm/high-power microwave heating. Journal of Food Engineering. 1999;40(1): $81-88$.

109. Campanone LA, Zaritzky NE. Mathematical analysis of microwave heating process. Journal of Food Engineering. 2005;69(3):359-368.

110. Acierno D, Barba A, d'Amore M. Heat transfer phenomena during processing materials with microwave energy. Heat and Mass Transfer. 2004;40(5):413-420.

111. Lin YE, Anantheswaran RC, Puri VM. Finite element analysis of microwave heating of solid foods. Journal of Food Engineering. 1995;25(1):85-112.

112. Zhou L, Puri VM, Anantheswaran RC, Yeh G. Finite element modeling of heat and mass transfer in food materials during microwave heating - model development and validation. Journal of Food Engineering. 1995;25(4):509-529.

113. Ayappa KG, Davis HT, Davis EA, Gordon J. Analysis of microwave heating of materials with temperature-dependent properties. AIChE Journal. 1991;37(3): 313-322.

114. Yee KS. Numerical solution of initial boundary value problems involving Maxwell's equations in isotropic media. IEEE Transactions on Antennas and Propagation. 1966;14(3):302-307.

115. Choi DH, Hoefer WJR. The finite difference time domain method and its application to eigenvalue problems. IEEE Transactions on Microwave Theory and Techniques. 1986;34(12):1464-1470.

116. Kashiwa T, Fukai I. A treatment by FDTD method of dispersive characteristics associated with electronic polarization. Microwave and Optics Technology Letters, 1990;3(6):203-205.

117. Taflove A. Review of the formulation and applications of the finite-difference time-domain method for numerical modeling of electromagnetic wave interactions with arbitrary structures. Wave Motion. 1988;10(6):547-582.

118. Taflove A, Umashankar KR. Review of numerical modeling of electromagnetic wave scattering and radar cross section. Proceedings of the IEEE. 1989;77(5): $682-689$.

119. Jurgens TG, Taflove A, Umashankar KR, Moore TG. Finite-difference timedomain modeling of curved surfaces. IEEE Transactions on Antennas and Propagation.1992;40(4):357-366. 
120. Yu W, Mittra R. A conformal FDTD software package modeling antennas and microstrip circuit components. IEEE Antennas and Propagation Magazine. 2000; 42(5):28-39.

121. Hwang S, Veronesi P, Leonelli C, Kim H. Forming silver conductive thick films by microwave heating. Journal of the American Ceramic Society. 2010;93(10): 3201-3205.

122. Poli G, Sola R, Veronesi P. Microwave-assisted combustion synthesis of NiAl intermetallics in a single mode applicator: Modeling and optimization. Materials Science and Engineering: A- Structural Materials: Properties, Microstructure and Processing. 2006;441(1-2):149-156.

123. Veronesi P, Leonelli C, Poli G, Garuti M. The design and optimization of a new microwave plasma source by numerical simulation. Plasma Devices and Operations. 2007;15(1):13-26.

124. Chatterjee S, Basak T, Das SK. Microwave driven convection in a rotating cylindrical cavity: a numerical study. Journal of Food Engineering. 2007;79(4): 1269-1279.

125. Peng Z, Hwang JY, Huang X, Andriese M, Bell W. Microwave field attenuation length and half-power depth in magnetic materials. TMS Annual Meeting, 2nd International Symposium on High-Temperature Metallurgical Processing - Held During the TMS 2011 Annual Meeting and Exhibition. 2011;51-57.

126. Peng Z, Hwang JY, Andriese M. Magnetic loss in microwave heating. Applied Physics Express. 2012;5(2):027304-027304-3.

127. Roy R, Peelamedu R, Grimes C, Cheng J, Agrawal D. Major phase transformations and magnetic property changes caused by electromagnetic fields at microwave frequencies. Journal of Materials Research. 2002;17(12):3008-3011.

128. Ma J, Diehl JF, Johnson EJ, Martin KR, Miskovsky NM, Smith CT, Weisel GJ, Weiss BL, Zimmerman DT. Systematic study of microwave absorption, heating, and microstructure evolution of porous copper powder metal compacts. Journal of Applied Physics. 2007;101(7):074906-071906-8.

129. Cao Z, Wang Z, Yoshikawa N, Taniguchi S. Microwave heating origination and rapid crystallization of PZT thin films in separated H field. Journal of Physics D: Applied Physics. 2008;41(9):092003-092003-4.

130. Cao Z, Yoshikawa N, Taniguchi S. Microwave heating behaviors of Si substrate 
materials in a single-mode cavity. Materials Chemistry and Physics. 2000;124(2-3):900-903.

131. Tanaka M, Kono H, Maruyama K. Selective heating mechanism of magnetic metal oxides by a microwave magnetic field. Physics Review B. 2009;79(10): 104420-104420-5.

132. Kato T, Yoshikawa N, Taniguchi S, Terakado Y, Ito N, Ohta M, Yoshizawa Y. Microwave magnetic field heating of a cobalt-based amorphous ribbon. Japanese Journal of Applied Physics. 2011;50(3):033001-033001-5.

133. Ayappa KG, Davis HT, Crapiste G, Davis EA, Gordon J. Microwave heating: an evaluation of power formulations. Chemical Engineering Science. 1991;46(4): 1005-1016.

134. Hossan MR, Byun DY, Dutta P. Analysis of microwave heating for cylindrical shaped objects. International Journal of Heat and Mass Transfer. 2010;53(23-24): 5129-5138.

135. Singh P, Babbar VK, Razdan A, Puri RK, Goel TC. Complex permittivity, permeability, and X-band microwave absorption of CaCoTi ferrite composites. Journal of Applied Physics. 2000;87(9):4362-4366.

136. Bahadoor A, Wang Y, Afsar MN. Complex permittivity and permeability of barium and strontium ferrite powders in $\mathrm{X}, \mathrm{KU}$, and K-band frequency ranges. Journal of Applied Physics. 2005;97(10):10F105-10F105-3.

137. Yan $\mathrm{M}, \mathrm{Hu}$ J. Microwave sintering of high-permeability $\left(\mathrm{Ni}_{0.20} \mathrm{Zn}_{0.60} \mathrm{Cu}_{0.20}\right) \mathrm{Fe}_{1.98} \mathrm{O}_{4}$ ferrite at low sintering temperatures. Journal of Magnetism and Magnetic Materials. 2006;305(1):171-176.

138. Sharma A, Afsar MN. Microwave complex permeability and permittivity measurements of commercially available nano-ferrites. IEEE Transactions on Magnetics. 2011;47(2):308-312.

139. Peng Z, Hwang JY, Park CL, Kim BG, Andriese M, Wang X. Microwave permittivity, permeability, and absorption capability of ferric oxide. ISIJ International. 2012;52(9):1541-1544.

140. Peng Z, Hwang JY, Mouris J, Hutcheon R, Sun X. Microwave absorption characteristics of conventionally heated nonstoichiometric ferrous oxide. Metallurgical and Materials Transactions A. 2011;42A(8):2259-2263.

141. Peng Z, Hwang JY, Zhang Z, Andriese M, Huang X. Thermal decomposition and 
regeneration of wüstite. TMS Annual Meeting, 3rd International Symposium on High-Temperature Metallurgical Processing - Held During the TMS 2012 Annual Meeting and Exhibition. 2012;147-156.

142. Peng Z, Hwang JY, Kim BG, Mouris J, Hutcheon R. Microwave absorption capability of high volatile bituminous coal during pyrolysis. Energy \& Fuels. 2012;26(8):5146-5151.

143. Evdokimov NI, Losev AP. Electrical conductivity and dielectric properties of solid asphaltenes. Energy and Fuels. 2010;24(7):3959-3969.

144. Gardner RFG, Sweett F, Tanner DW. The electrical properties of alpha ferric oxide-I.: the impure oxide. Journal of Physics and Chemistry of Solids. 1963; 24(10):1175-1181.

145. Wilson NC, Russo SP. Hybrid density functional theory study of the highpressure polymorphs of $\alpha-\mathrm{Fe}_{2} \mathrm{O}_{3}$ hematite. Physical Review B. 2009;79(9): 094113-094113-9.

146. Cavaliere-Jaricot S, Brioude A, Miele P. Ultra-thin polycrystalline hematite and goethite-hematite core-shell nanorods. Langmuir. 2009;25(5):2551-2553.

147. Morais PC, Lima ECD, Rabelo D, Reis AC, Pelegrini F. Magnetic resonance of magnetite nanoparticles dispersed in mesoporous copolymer matrix. IEEE Transactions on Magnetics. 2000;36(5):3038-3040.

148. Andersson B, Sletnes JO. Decomposition and ordering in $\mathrm{Fe}_{1-\mathrm{x}} \mathrm{O}$. Acta Crystallographica Section A: Crystal Physics, Diffraction, Theoretical and General Crystallography. 1977;33(2):268-276.

149. Broussard L. The disproportionation of wustite. Journal of Physical Chemistry. 1969;73(6):1848-1854.

150. Shechter H, Hillman P. Mössbauer study of the structure and decomposition of wustite. Journal of Applied Physics. 1966;37(8):3043-3047.

151. Zhang D, Weng G, Gong S, Zhou D. Computer simulation of grain growth of intermediate-and final-stage sintering and Ostwald ripening of $\mathrm{BaTiO}_{3}$-based PTCR ceramics. Materials Science and Engineering: B-Advanced Functional Solid-State Materials. 2003;99(1):428-432.

152. Thompson ED, Wohlfarth EP, Bryan AC, The low temperature variation of the saturation magnetization of ferromagnetic metals and alloys. Proceedings of the Physical Society. 1964;83(1):59-70. 
153. Seki I, Nagata K. Reduction kinetics of hematite powder mechanically milled with graphite. ISIJ International. 2006;46(1):1-7.

154. Yang J, Mori T, Kuwabara M. Mechanism of carbothermic reduction of hematite in hematite-carbon composite pellets. ISIJ International. 2007;47(10):1394-1400.

155. Ishizaki K, Nagata K. Microwave induced solid-solid reactions between $\mathrm{Fe}_{3} \mathrm{O}_{4}$ and carbon black powders. ISIJ International. 2008;48(9):1159-1164.

156. Yin M, Chen Z, Deegan B, O’Brien S. Wüstite nanocrystals: synthesis, structure and superlattice formation. Journal of Materials Research. 2007;22(7):1987-1995.

157. Gheisari M, Mozaffari M, Acet M, Amighian J. Preparation and investigation of magnetic properties of wüstite nanoparticles. Journal of Magnetism and Magnetic Materials. 2008;320(21):2618-2621.

158. Mozaffari M, Gheisari M, Niyaifar M, Amighian J. Magnetic properties of mechanochemically prepared iron-wüstite $\left(\mathrm{Fe}-\mathrm{Fe}_{\mathrm{y}} \mathrm{O}\right)$ nanocomposites. Journal of Magnetism and Magnetic Materials. 2009;321(19):2981-2984.

159. Takacs L. Metal-metal oxide systems for nanocomposite formation by reaction milling. Nanostructured Materials. 1993;2(3):241-249.

160. Ding J, Miao WF, Pirault E, Street R, McCormick PG. Mechanical alloying of iron-hematite powders. Journal of Alloys and Compounds. 1998;267(1-2):199-204.

161. Tokumitsu K, Nasu T. Preparation of lamellar structured alpha-Fe $/ \mathrm{Fe}_{3} \mathrm{O}_{4}$ complex particle by thermal decomposition of wüstite. Scripta Materialia. 2001; 44(8-9):1421-1424.

162. Yue L, Shui M, Xu Z. The decomposition kinetics of nanocrystalline calcite. Thermochimica Acta. 1999;335(1-2):121-126.

163. Dollimore D, Tong $\mathrm{P}$, Alexander KS. The kinetic interpretation of the decomposition of calcium carbonate by use of relationships other than the Arrhenius equation. Thermochimica Acta. 1996;282/283(SPEC. ISS.): 13-27.

164. Ninan KM, Krishnan K, Krishnamurthy VN. Kinetics and mechanism of thermal decomposition of in situ generated calcium carbonate. Journal of Thermal Analysis and Calorimetry. 1991;37(7):1533-1543.

165. Asirvatham BJ, Munir ZA. The decomposition of cadmium carbonate in air and 
in vacuum. Journal of Materials Science. 1986;21(6):1997-2001.

166. Maitra S, Choudhury A, Das HS, Pramanik MsJ. Effect of compaction on the kinetics of thermal decomposition of dolomite under non-isothermal condition. Journal of Materials Science. 2005;40(18):4749-4751.

167. Vlaev LT, Georgieva VG, Gospodinov GG. Kinetics of isothermal decomposition of $\mathrm{ZnSeO}_{3}$ and $\mathrm{CdSeO}_{3}$. Journal of Thermal Analysis and Calorimetry. 2005;79(1):163-168.

168. Brown ME. The Prout-Tompkins rate equation in solid-state kinetics. Thermochimica Acta. 1997;300(1-2):93-106.

169. Palanisamy T, Gopalakrishnan J, Viswanathan B, Srinivasan V, Sastri MVC. Kinetics of thermal decomposition of some metal oxalates. Thermochimica Acta. 1971;2(3):265-273.

170. Wendlandt WW, George TD, Horton GR. The thermal decomposition of thorium(IV), uranium(IV), and the rare-earth metal(III) oxalate hydrates. Differential thermal analysis and weight-loss studies. Journal of Inorganic and Nuclear Chemistry. 1961;17(3-4):273-280.

171. Subramanian MS, Singh RN, Sharma HD. Reaction kinetics of some actinide oxalates by differential thermal analysis. Journal of Inorganic and Nuclear Chemistry. 1969;31(12):3789-3795.

172. Órfão JJM, Martins FG. Kinetic analysis of thermogravimetric data obtained under linear temperature programming-a method based on calculations of the temperature integral by interpolation. Thermochimica Acta. 2002;390(1-2):195-211.

173. Coats AW, Redfern JP. Kinetic parameters from thermogravimetric data. Nature. 1964;201(1):68-69.

174. Bamzai KK, Kumar S. Studies on kinetics and mechanism of thermal decomposition of yttrium tartrate trihydrate crystals. Materials Chemistry and Physics. 2008;107(2-3):200-207.

175. Šesták J, Berggren G. Study of the kinetics of the mechanism of solid-state reactions at increasing temperatures. Thermochimica Acta. 1971;3(1):1-12.

176. Malek J, Criado JM. Is the šesták-berggren equation a general expression of kinetic models? Thermochimica Acta. 1991;75(2):305-309. 
177. Šesták J, Satava V, Wendlandt WW. The study of heterogeneous processes by thermal analysis. hermochimica Acta. 1973;7(5):333-556.

178. Vlaev LT, Markovska IG, Lyubchev LA. Non-isothermal kinetics of pyrolysis of rice husks. Thermochimica Acta. 2003;406(1-2):1-7.

179. Vlaev LT, Nikolova MM, Gospodinov GG. Non-isothermal kinetics of dehydration of some selenite hexahydrates. Journal of Solid State Chemistry. 2004;177(8):2663-2669.

180. Laidler KJ, King MC. The development of transition-state theory. Journal of Physical Chemistry. 1983;87(15):2657-2664.

181. Vlaev LT, Nikolova MM, Gospodinov GG. Study on the kinetics of nonisothermal dehydration of alkaline earth metal selenites. Monatshefte für Chemie/ Chemical Monthly. 2005;136(9):1553-1566.

182. Maitra S, Bandyopadhyay N, Das S. Non-isothermal decomposition kinetics of alkaline earth metal carbonates. Journal of the American Ceramic Society. 2007; 90(4):1299-1303.

183. Baxter LL, Fletcher TH, Ottesen DK. Spectral emittance measurements of coal particles. Energy and Fuels. 1988;2(4):423-430.

184. Saikia BK, Boruah RK, Gogoi PK. FT-IR and XRD analysis of coal from. Makum coalfield of Assam. Journal Of Earth System Science. 2007;116(6):575-579.

185. Das TK. Evolution characteristics of gases during pyrolysis of maceral concentrates of Russian coking coals. Fuel. 2001;80(4):489-500.

186. Peng Z, Hwang JY, Andriese M, Zhang Z, Huang X. Heat transfer characteristics of magnetite under microwave irradiation. TMS Annual Meeting, Extraction and Processing Division - 2012 EPD Congress - Held During the TMS 2012 Annual Meeting and Exhibition. 2012;121-128.

187. Hao Y, Mittra R. FDTD Modeling of Metamaterials: Theory and Applications. Artech House Publishers, Boston, MA, 2008.

188. Sullivan DM. Electromagnetic Simulation Using the FDTD Method, Wiley-IEEE Press, Piscataway, NJ, 2000.

189. Jacob J, Chia LHL, Boey FYC. Thermal and non-thermal interaction of microwave radiation with materials. Journal of Materials Science. 
1995;30(21):5321-5327.

190. Haswell SJ; Howarth N. Perturbation of a solid phase separation process by a non-thermal microwave effect. Analytica Chimica Acta. 1999;387(2):113-120.

191. de la Hoz A, Díaz-Ortiz A, Moreno A. Microwaves in organic synthesis. Thermal and non-thermal microwave effects. Chemical Society Reviews. 2005;34(2):164-178.

192. George DF, Bilek MM, McKenzie DR. Non-thermal effects in the microwave induced unfolding of proteins observed by chaperone binding. Bioelectromagnetics. 2008;29(4):324-330.

193. Shazman A, Mizrahi S, Cogan U, Shimoni E. Examining for possible nonthermal effects during heating in a microwave oven. Food Chemistry. 2007;103(2):444-453.

194. Li H, Liao L, Liu L. Kinetic investigation into the non-thermal microwave effect on the ring-opening polymerization of $\varepsilon$-caprolactone. Macromolecular Rapid Communications. 2007;28(4):411-416.

195. Herrero MA, Kremsner JM, Kappe CO. Nonthermal microwave effects revisited: on the importance of internal temperature monitoring and agitation in microwave chemistry. Journal of Organic Chemistry. 2008;73(1):36-47.

196. Razzaq T, Kremsner JM, Kappe CO. Investigating the existence of nonthermal/specific microwave effects using silicon carbide heating elements as power modulators. Journal of Organic Chemistry. 2008;73(16):6321-6329.

197. Selvaraj DK. Microwave Assisted Chemical Synthesis Using Process-intensified Reactors. Ph.D. dissertation. Clarkson University, Potsdam, New York, 2009.

198. Kanno M, Nakamura K, Kanai E, Hoki K, Kono H, Tanaka M. Theoretical verification of nonthermal microwave effects on intramolecular reactions. Journal of Physical Chemistry A. 2012;116(9):2177-2183.

199. Peng Z, Hwang JY, Andriese M, Bell W, Huang X, Wang X. Numerical simulation of heat transfer during microwave heating of magnetite. ISIJ International. 2011;51(6):884-888.

200. Peng Z, Hwang JY, Park CL, Kim BG, Onyedika G. Numerical analysis of heat transfer characteristics in microwave heating of magnetic dielectrics. Metallurgical and Materials Transactions A. 2012;43A(3):1070-1078. 
201. Mølgaard J, Smeltzer WW. Thermal conductivity of magnetite and hematite. Journal of Applied Physics. 1971;42(9):3644-3647.

202. Westrum Jr EF, Grønvold F. Magnetite $\left(\mathrm{Fe}_{3} \mathrm{O}_{4}\right)$ heat capacity and thermodynamic properties from 5 to $350 \mathrm{~K}$, low-temperature transition. Journal of Chemical Thermodynamics. 1969;1(6):543-557.

203. Sprague AL, Roush TL, Downs RT, Righter K. Response to comment on "Comparison of laboratory emission spectra with mercury telescopic data" by Melissa Lane. Icarus. 2000;143(2):409-411.

204. McGill SL, Walkiewicz JW, Smyres GA. The effects of power level on the microwave heating of selected chemicals and minerals. MRS Proceedings. 1988; $124: 247-252$.

205. Jiang $\mathrm{Y}$, Zhu Y, Cheng G. Synthesis of $\mathrm{Bi}_{2} \mathrm{Se}_{3}$ nanosheets by microwave heating using an ionic liquid. Crystal Growth \& Design. 2006;6(9):2174-2176.

206. Chatterjee S, Basak T, Das SK. Microwave driven convection in a rotating cylindrical cavity: A numerical study. Journal of Food Engineering. 2007;79(4): 1269-1279.

207. Yang HW, Gunasekaran S. Comparison of temperature distribution in model food cylinders based on Maxwell's equations and Lambert's law during pulsed microwave heating. Journal of Food Engineering. 2004;64(4):445-453.

208. Peng Z, Hwang JY, Kim BG, Andriese M, Wang X. Microwave reflection loss of ferric oxide. TMS Annual Meeting, 4th International Symposium on HighTemperature Metallurgical Processing - Held During the TMS 2013 Annual Meeting and Exhibition. 2013; to be published.

209. Rattanadecho P, Klinbun W. Theoretical analysis of microwave heating of dielectric materials filled in a rectangular waveguide with various resonator distances. Journal of Heat Transfer. 2011;133(3):031008-031008-10.

210. Buchelnikov VD, Louzguine-Luzgin DV, Xie G, Li S, Yoshikawa N, Sato M, Anzulevich AP, Bychkov IV, Inoue A. Heating of metallic powders by microwaves: Experiment and theory. Journal of Applied Physics. 2008;104(11): 113505-113505-10.

211. Agrawal D. Latest global developments in microwave materials processing. Materials Research Innovations. 2010;14(1):3-8.

212. Martin LP, Dadon D, Rosen M, Gershon D, Rybakov KI, Birman A, Calame JP, 
Levush B, Carmel Y, Hutcheon R. Effects of anomalous permittivity on the microwave heating of zinc oxide. Journal of Applied Physics. 1998;83(1):432-437.

213. Rybakov KI, Semenov VE, Egorov SV, Eremeev AG, Plotnikov IV, Bykov Yu $\mathrm{U}$. Microwave heating of conductive powder materials. Journal of Applied Physics. 2006;99(2):023506-023506-9.

214. Robinson J, Kingman S, Irvine D, Licence P, Smith A, Dimitrakis G, Obermayer $\mathrm{D}$, Kappe CO. Understanding microwave heating effects in single mode type cavities-theory and experiment. Physical Chemistry Chemical Physics. 2010; 12(18):4750-4758.

215. Shen G, Xu Z, Li Y. Absorbing properties and structural design of microwave absorbers based on $\mathrm{W}$-type La-doped ferrite and carbon fiber composites. Journal of Magnetism and Magnetic Materials. 2006;301(2):325-330.

216. Shi X, Cao M, Yuan J, Fang X. Dual nonlinear dielectric resonance and nesting microwave absorption peaks of hollow cobalt nanochains composites with negative permeability. Applied Physics Letters. 2009;95(16):163108-163108-3.

217. Song W, Cao M, Hou Z, Yuan J, Fang X. High-temperature microwave absorption and evolutionary behavior of multiwalled carbon nanotube nanocomposite. Scripta Materialia. 2009;61(2):201-204.

218. Cao M, Song W, Hou Z, Wen B, Yuan J. The effects of temperature and frequency on the dielectric properties, electromagnetic interference shielding and microwave-absorption of short carbon fiber/silica composites. Carbon. 2010; 48(3):788-796.

219. Peng Z, Hwang JY, Andriese M. Absorber impedance matching in microwave heating. Applied Physics Express. 2012;5(7):077301-077301-3.

220. Cao M, Wang B, Li Q, Yuan J, Xu G, Qin S, Fang X. Towards an intelligent $\mathrm{CAD}$ system for multilayer electromagnetic absorber design. Materials and Design. 1998;19(3):113-120.

221. Michielssen E, Sajer JM, Ranjithan S, Mittra R. Design of lightweight, broadband microwave absorbers using genetic algorithms. IEEE Transactions on Microwave Theory and Techniques. 1993;41(6/7):1024-1031.

222. Cao M, Zhu J, Yuan J, Zhang T, Peng Z, Gao Z, Xiao G, Qin S. Computation design and performance prediction towards a multi-layer microwave absorber. Materials and Design. 2002;23(6):557-564. 
223. Cao M, Qin R, Qiu C, Zhu J. Matching design and mismatching analysis towards radar absorbing coatings based on conducting plate. Materials and Design. 2003;24(5):391-396.

224. Cui S, Weile D, Volakis J. Novel planar electromagnetic absorber designs using genetic algorithms. IEEE Transactions on Antennas and Propagation. 2006; 54(6):1811-1817.

225. Goudos SK, Sahalos JN. Microwave absorber optimal design using multiobjective particle swarm optimization. Microwave and Optical Technology Letters. 2006;48(8):1553-1558.

226. Yuan J, Xiao G, Cao M. A novel method of computation and optimization for multi-layered radar absorbing coatings using open source software. Materials and Design. 2006;27(1):45-52.

227. Jiang L, Li X. Design high performance microwave absorbers using adaptive genetic algorithm (AGA). Asia-Pacific Symposium on Electromagnetic Compatibility and 19th International Zurich Symposium on Electromagnetic Compatibility, 2008. APEMC 2008. 2008;758-761.

228. Goudos SK. Design of microwave broadband absorbers using a self-adaptive differential evolution algorithm. International Journal of RF and Microwave Computer-Aided Engineering. 2008;19(3):364-372.

229. Jiang L, Cui J, Shi L, Li X. Pareto optimal design of multilayer microwave absorbers for wide-angle incidence using genetic algorithms. IET Microwaves, Antennas \& Propagation. 2009;3(4):572-579.

230. Jiang L, Li X, Zhang J. Design of high performance multilayer microwave absorbers using fast Pareto genetic algorithm. Science in China Series E: Technological Sciences. 2009;52(9):2749-2757.

231. Huang Y, Yuan J, Song W, Wen B, Fang X, Cao M. Microwave absorbing materials: Solutions for real functions under ideal conditions of microwave absorption. Chinese Physics Letters. 2010;27(2):027702-027702-4.

232. Ma Z, Cao C, Liu Q, Wang J. A new method to calculate the degree of electromagnetic impedance matching in one-layer microwave absorbers. Chinese Physics Letters. 2012;29(3):038401-038401-4.

233. Dib NI, Asi M, Sabbah A. On the optimal design of multilayer microwave absorbers. Progress in Electromagnetics Research C. 2010;13:171-185. 
234. Asi M, Dib NI, Design of multilayer microwave broadband absorbers using central force optimization. Progress in Electromagnetics Research B. 2010;26: $101-113$.

235. Ding F, Cui Y, Ge X, Jin Y, He S. Ultra-broadband microwave metamaterial absorber. Applied Physics Letters. 2012;100(10):103506-103506-4. 


\section{Appendix A-1 Copyright Permission for Chapter 3}

A part of Chapter 3 was originally published in ISIJ International (an Iron and Steel Institute of Japan journal). Permission has been granted to the author who reused the published work in his Ph.D. dissertation. A copy of the permission is attached below.

Title: Microwave Penetration Depth in Materials with Non-zero Magnetic Susceptibility

Authors: Zhiwei Peng, Jiann-Yang Hwang, Joe Mouris, Ron Hutcheon, Xiaodi Huang.

Publication: ISIJ International

Year: $\quad 2010$

Volume: $\quad 50$

Issue: $\quad 11$

Pages: $\quad$ 1590-1596

Copyright: $\quad$ C 2010 The Iron and Steel Institute of Japan 
To: ISIJ International

\section{From Zhiwei Peng}

\section{Request for permission to reproduce copyrighted material}

Dear ISIJ International Editor,

I am preparing a review article entitled:

Heat Transfer in Microwave Heating (A Doctoral Dissertation)

For submission to Graduate School of Michigan Technological University

published by Michigan Technological University

I wish to have your permission to include in my article the following material:

\section{Full text}

From the article written by Zhiwei Peng, Jiann-Yang Hwang, Joe Mouris, Ron Hutcheon and Xiaodi Huang.

in the publication "Microwave Penetration Depth in Materials with Non-zero Magnetic Susceptibility" ISIJ Int., 50 (2010), 1590.

If permission is granted for the use of this material, the author(s) and your publication will be credited as the source. If you would like the credit line to take any special form, please let me know what this should be.

I should be greatly appreciated if you would indicate your agreement by signing and returning one copy this form. Thank you for your cooperation.

Sincerely yours,

Name_Z Zhiwei Peng

Fax +1-9064872934

E-mail_zpeng@mtu.edu

Permission granted to reproduce the material specified above:

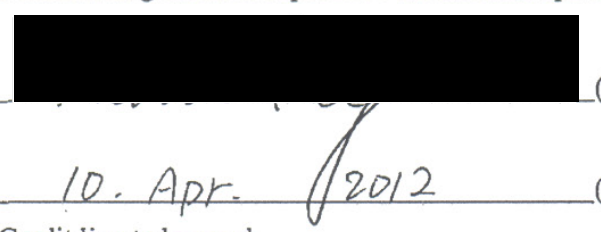

(Signature of copyright holder/author) (Date)

Credit line to be used: 


\section{Appendix A-2 Copyright Permission for Chapter 3}

A part of Chapter 3 was originally published in TMS Annual Meeting, 2nd International Symposium on High-Temperature Metallurgical Processing - Held During the TMS 2011 Annual Meeting and Exhibition (a Minerals, Metals and Materials Society conference proceedings). According to the regulations in the Copyright Form of the Minerals, Metals and Materials Society, the author retains the right to reproduce the published work in his Ph.D. dissertation. The Copyright Form can be accessed at the following website: http://www.tms.org/pubs/books/instructions/Copyright_Form.pdf. A copy of the Rights of Authors in the Copyright Form is attached below.

Title: $\quad$ Microwave Field Attenuation Length and Half-power Depth in Magnetic Materials

Authors: Zhiwei Peng, Jiann-Yang Hwang, Xiaodi Huang, Matthew Andriese, Wayne Bell.

Publication: TMS Annual Meeting, 2nd International Symposium on HighTemperature Metallurgical Processing - Held During the TMS 2011 Annual Meeting and Exhibition

Year: $\quad 2011$

Pages: $\quad 51-57$

Copyright: (C) 2011 The Minerals, Metals and Materials Society 


\section{PART A. COPYRIGHT TRANSFER}

Copyright, title, interest, and all right in the manuscript named above is hereby transferred to TMS, effective when the manuscript is accepted for publication. This assignment and transfer applies to any other publication of the Society in addition to the publication designated.

THE AUTHOR(S), OR THE EMPLOYER(S) IN THE CASE OF WORKS MADE FOR HIRE, RETAIN THE FOLLOWING RIGHTS:

1.) All proprietary rights, other than copyright, such as patent rights.

2.) The right to use all or portions of the above paper in oral presentations or other works.

3.) The right to make limited distribution of the article or portions thereof prior to publication.

4.) Royalty-free permission to reproduce the above paper for personal use or, in the case of a work made for hire, the employer's use, provided that a.) the source and TMS copyright are indicated, b.) the copies are not used in a way that implies endorsement by TMS of a product or service, and c.) the copies are not offered for sale.

5.) In the case of work performed under U.S. government contract, TMS grants the U.S. government royalty-free permission to reproduce all or portions of the paper, and to authorize others to do so for U.S. government purposes. 


\section{Appendix B-1 Copyright Permission for Chapter 4}

The content of Chapter 4 was originally published in Applied Physics Express (a Japan Society of Applied Physics journal). According to the Copyright Transfer Agreement of the Japan Society of Applied Physics, the author retains the right to reproduce the published work in his Ph.D. dissertation. The Copyright Transfer Agreement can be accessed at the following website: http://apex.jsap.jp/pdf/copyrightform.pdf. A copy of the "Rights of Authors"' in the Copyright Transfer Agreement is attached below.

Title: $\quad$ Magnetic Loss in Microwave Heating

Authors: $\quad$ Zhiwei Peng, Jiann-Yang Hwang, Matthew Andriese

Publication: Applied Physics Express

Year: $\quad 2012$

Volume: $\quad 5$

Issue: $\quad 2$

Pages: $\quad$ 027304-027304-3

Copyright: $\quad$ C 2012 The Japan Society of Applied Physics 


\section{Copyright Transfer Agreement}

The undersigned hereby agree(s) to transfer the following Economic Rights to the Work to the Japan Society of Applied Physics (hereinafter referred to as "JSAP"), effective if and when the manuscript is accepted for publication by APEX Editorial Board.

Economic Rights: any and all rights including, but not limited to, right of reproduction (Article 21 of the Copyright Law of Japan (hereinafter referred to as the "Law")), right of performance (Article 22 of the Law), right of presentation (Article 22-2 of the Law), right of public transmission (Article 23 of the Law), right of recitation (Article 24 of the Law), right of exhibition (Article 25 of the Law), right of distribution (Article 26 of the Law), right of transfer of ownership (Article 26-2 of the Law), right of lending (Article 26-3 of the Law), right of translation and adaptation (Article 27 of the Law), and the right of the original Author regarding the exploitation of derivative works (Article 28 of the Law).

The Author(s) shall not exercise Moral Rights with respect to the Society. Moral Rights refer to the right to make the works public (Article 18 of the Law), right to determine the indication of the Author's name (Article 19 of the Law), right to preserve the integrity of the work (Article 20 of the Law).

The Author(s) retain(s) the following rights (the "Rights of Authors"'):

Rights of Author(s)

(1) The right to use a part of the Work in future works and derivatives prepared by or on behalf of the Author(s). 
(2) The right to make copies of all or part of the Work without further permission, for the purpose of his personal use but not for resale.

Author(s) shall not use any Economic Rights other than listed above without prior written consent of JSAP.

Notwithstanding the foregoing, the Author may use, translate, or adapt the manuscript without prior written consent of JSAP, to the extent that such use falls within the limitations (i.e., Articles 30 through 50 of the Law), except in the cases listed below:

\section{(A) Commercial purposes;}

(B) Duplicated or redundant contributions to other journals of an academic society or academic journals or communications, whatsoever, domestic or overseas; or

(C) Any other purpose deemed extravagant in light of fair academic practices.

(3) Exception. - The submitted manuscript has been authored by a contractor of the U.S. Government under Contract No.

Accordingly, the U.S. Government retains a nonexclusive royalty-free license to publish or reproduce the published form of this contribution, or allows others to do so, for U.S. Government purposes. Note that the rights the U.S. Government retains are not for commercial purposes.

For further information about The Copyright Policy of JSAP, see http://www.jsap.or.jp/english/link/copyright.html. 


\section{Appendix C-1 Copyright Permission for Chapter 5}

A part of Chapter 5 was originally published in ISIJ International (an Iron and Steel Institute of Japan journal). Permission has been granted to the author who reused the published work in his Ph.D. dissertation. A copy of the permission is attached below.

Title: Microwave Permittivity, Permeability, and Absorption Capability of Ferric Oxide

Authors: Zhiwei Peng, Jiann-Yang Hwang, Chong-Lyuck Park, Byoung-Gon Kim, Matthew Andriese, Xinli Wang.

Publication: ISIJ International

Year: $\quad 2012$

Volume: $\quad 52$

Issue: $\quad 9$

Pages: $\quad$ 1541-1544

Copyright: $\quad$ C 2012 The Iron and Steel Institute of Japan 
Dear Mr. Zhiwei Peng,

Zhiwei Peng, Jiann-Yang Hwang, Chong-Lyuck Park, Byoung-Gon Kim, Matthew, Andriese and Xinli Wang ISIJ Int., 52 (2012), 1541 .

We hereby grant permission of the use of the materials requested above.

\section{Copyright Notice:}

You are requested to write the credit line "Originally published in ISIJ International " at foot of your publication.

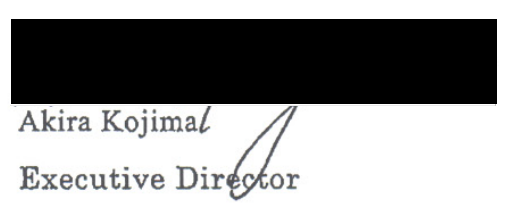

The Iron and Steel Institute of Japan 


\section{Appendix C-2 Copyright Permission for Chapter 5}

A part of Chapter 5 was originally published in ISIJ International (an Iron and Steel Institute of Japan journal). Permission has been granted to the author who reused the published work in his Ph.D. dissertation. A copy of the permission is attached below.

Title: Microwave Penetration Depth in Materials with Non-zero Magnetic Susceptibility

Authors: Zhiwei Peng, Jiann-Yang Hwang, Joe Mouris, Ron Hutcheon, Xiaodi Huang.

Publication: ISIJ International

Year: $\quad 2010$

Volume: $\quad 50$

Issue: $\quad 11$

Pages: $\quad$ 1590-1596

Copyright: $\quad$ C 2010 The Iron and Steel Institute of Japan 
To: ISIJ International

From Zhiwei Peng

Request for permission to reproduce copyrighted material

Dear ISIJ International Editor,

I am preparing a review article entitled;

Heat Transfer in Microwave Heating (A Doctoral Dissertation)

For submission to Graduate School of Michigan Technological University

published by Michigan Technological University

I wish to have your permission to include in my article the following material:

Full text

From the article written by Zhiwei Peng, Jiann-Yang Hwang, Joe Mouris, Ron Hutcheon and Xiaodi Huang.

in the publication "Microwave Penetration Depth in Materials with Non-zero Magnetic Susceptibility" ISIJ Int. 50 (2010), 1590.

If permission is granted for the use of this material, the author(s) and your publication will be credited as the source. If you would like the credit line to take any special form, please let me know what this should be.

I should be greatly appreciated if you would indicate your agreement by signing and returning one copy this form. Thank you for your cooperation.

Sincerely yours,
\begin{tabular}{l} 
Name_Zhiwei Peng \\
Fax $\quad+1-9064872934$ \\
\hline E-mail zpeng@mtu.edu
\end{tabular}

Permission granted to reproduce the material specified above:

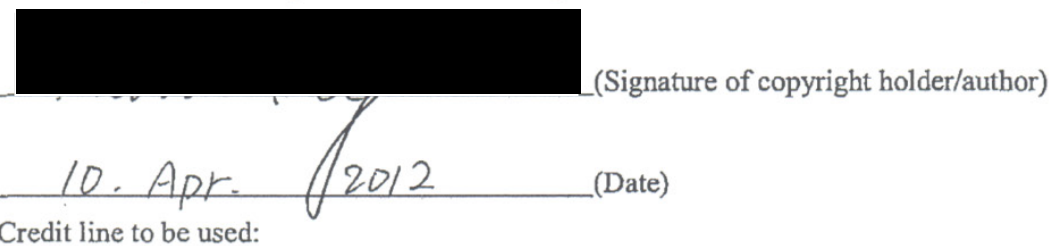

Credit line to be used: 


\section{Appendix C-3 Copyright Permission for Chapter 5}

A part of Chapter 5 was originally published in Metallurgical and Materials Transactions A (a Minerals, Metals and Materials Society journal). According to the regulations in the Copyright Form of the Minerals, Metals and Materials Society, the author retains the right to reproduce the published work in his $\mathrm{Ph}$.D. dissertation. A copy of the Rights of Authors in the Copyright Form is attached below.

Title: Microwave Absorption Characteristics of Conventionally Heated Nonstoichiometric Ferrous Oxide

Authors: Zhiwei Peng, Jiann-Yang Hwang, Joe Mouris, Ron Hutcheon, Xiang Sun.

Publication: Metallurgical and Materials Transactions A

Year: $\quad 2011$

Volume: $\quad 42 \mathrm{~A}$

Issue: $\quad 8$

Pages: 2259-2263

Copyright: $\quad$ (C) 2011 The Minerals, Metals and Materials Society 


\section{PART A. COPYRIGHT TRANSFER}

Copyright, title, interest, and all rights in the manuscript named above are hereby transferred to TMS and ASM International, effective when the manuscript is accepted for publication. This assignment and transfer applies to any other subsequent publication of either organization in addition to the publication designated, provided that proper acknowledgement is made.

THE AUTHOR(S), OR THE EMPLOYER(S) IN THE CASE OF WORKS MADE FOR HIRE, RETAIN THE FOLLOWING RIGHTS:

1.) All proprietary rights, other than copyright, such as patent rights.

2.) The right to use all or portions of the above paper in oral presentations or other works.

3.) The right to make limited distribution of the article or portions thereof prior to publication.

4.) Royalty-free permission to reproduce the above paper for personal use or, in the case of a work made for hire, the employer's use, provided that a.) the source and copyright are indicated, b.) the copies are not used in a way that implies endorsement by TMS and ASM International of a product or service, and c.) the copies are not offered for sale.

5.) In the case of work performed under U.S. government contract, TMS and ASM International grants the U.S. government royalty-free permission to reproduce all or portions of the paper, and to authorize others to do so for U.S. government purposes. 


\section{Appendix C-4 Copyright Permission for Chapter 5}

A part of Chapter 5 was originally published in TMS Annual Meeting, 3rd International Symposium on High-Temperature Metallurgical Processing - Held During the TMS 2012 Annual Meeting and Exhibition (a Minerals, Metals and Materials Society conference proceedings). According to the regulations in the Copyright Form of the Minerals, Metals and Materials Society, the author retains the right to reproduce the published work in his Ph.D. dissertation. The Copyright Form can be accessed at the following website: http://www.tms.org/pubs/books/instructions/Copyright_Form.pdf. A copy of the Rights of Authors in the Copyright Form is attached below.

Title: $\quad$ Thermal Decomposition and Regeneration of Wüstite

Authors: Zhiwei Peng, Jiann-Yang Hwang, Zheng Zhang, Matthew Andriese, Xiaodi Huang

Publication: TMS Annual Meeting, 3rd International Symposium on HighTemperature Metallurgical Processing - Held During the TMS 2012 Annual Meeting and Exhibition

Year: $\quad 2012$

Pages: $\quad 147-156$

Copyright: $\quad 2012$ The Minerals, Metals and Materials Society 


\section{PART A. COPYRIGHT TRANSFER}

Copyright, title, interest, and all right in the manuscript named above is hereby transferred to TMS, effective when the manuscript is accepted for publication. This assignment and transfer applies to any other publication of the Society in addition to the publication designated.

THE AUTHOR(S), OR THE EMPLOYER(S) IN THE CASE OF WORKS MADE FOR HIRE, RETAIN THE FOLLOWING RIGHTS:

1.) All proprietary rights, other than copyright, such as patent rights.

2.) The right to use all or portions of the above paper in oral presentations or other works.

3.) The right to make limited distribution of the article or portions thereof prior to publication.

4.) Royalty-free permission to reproduce the above paper for personal use or, in the case of a work made for hire, the employer's use, provided that a.) the source and TMS copyright are indicated, b.) the copies are not used in a way that implies endorsement by TMS of a product or service, and c.) the copies are not offered for sale.

5.) In the case of work performed under U.S. government contract, TMS grants the U.S. government royalty-free permission to reproduce all or portions of the paper, and to authorize others to do so for U.S. government purposes. 


\section{Appendix C-5 Copyright Permission for Chapter 5}

A part of Chapter 5 in this dissertation was originally published in Energy \& Fuels (An American Chemical Society Journal). According to the Journal Publishing Agreement of the American Chemical Society, the author retains the right to reproduce the published work in his Ph.D. dissertation. The Journal Publishing Agreement of the American Chemical Society can be accessed at the following website: http://pubs.acs.org/paragonplus/copyright/jpa_form_a.pdf. A copy of the "Permitted Uses by Author(s)" in the Journal Publishing Agreement is attached below.

Title: Microwave Absorption Capability of High Volatile Bituminous Coal during Pyrolysis.

Authors: Zhiwei Peng, Jiann-Yang Hwang, Byoung-Gon Kim, Joe Mouris, Ron Hutcheon.

Publication: Energy \& Fuels

Year: $\quad 2012$

Volume: $\quad 26$

Issue: $\quad 8$

Pages: $\quad$ 5146-5151

Copyright: $\quad$ C 2012 The American Chemical Society 


\section{AMERICAN CHEMICAL SOCIETY \\ JOURNAL PUBLISHING AGREEMENT}

Form A: Authors Who Hold Copyright and Works-for-Hire

Control \#2011-10-11

\section{SECTION I: Copyright}

1. Submitted Work: The Corresponding Author, with the consent of all coauthors, hereby transfers to the ACS the copyright ownership in the referenced Submitted Work, including all versions in any format now known or hereafter developed. If the manuscript is not accepted by ACS or withdrawn prior to acceptance by ACS, this transfer will be null and void.

2. Supporting Information: The copyright ownership transferred to ACS in any copyrightable* Supporting Information accompanying the Submitted Work is nonexclusive. The Author and the ACS agree that each has unlimited use of Supporting Information. Authors may use or authorize the use of material created by the Author in the Supporting Information associated with the Submitted or Published Work for any purpose and in any format.

*Title 17 of the United States Code defines copyrightable material as "original works of authorship fixed in any tangible medium of expression" (Chapter 1, Section 102). To learn more about copyrightable material see "Frequently Asked Questions about Copyright" on the Publications Division website, at http://pubs.acs.org/page/copyright/learning_module/module.html. 
SECTION II: Permitted Uses by Author(s)

1. Reuse/Republication of the Entire Work in Theses or Collections:

Authors may reuse all or part of the Submitted, Accepted or Published Work in a thesis or dissertation that the Author writes and is required to submit to satisfy the criteria of degree-granting institutions. Such reuse is permitted subject to the ACS' "Ethical Guidelines to Publication of Chemical Research" (http://pubs.acs.org/ethics); the Author should secure written confirmation (via letter or email) from the respective ACS journal editor(s) to avoid potential conflicts with journal prior publication**/embargo policies. Appropriate citation of the Published Work must be made. If the thesis or dissertation to be published is in electronic format, a direct link to the Published Work must also be included using the ACS Articles on Request author-directed link (see http://pubs.acs.org/page/policy/articlesonrequest/index.html).

Authors also may reuse the Submitted, Accepted, or Published work in printed collections that consist solely of the Author's own writings; if such collections are to be posted online or published in an electronic format, please contact ACS at copyright@acs.org to inquire about terms for licensed electronic use.

2. Reuse of Figures, Tables, Artwork, and Text Extracts in Future Works: Authors may reuse figures, tables, artwork, illustrations, text extracts of up to 400 words, and data from the Author's Submitted, Accepted, or Published Work in which the ACS holds copyright for teaching or training purposes, in presentations at conferences and seminars, in subsequent scholarly publications of which they are an Author, and for posting on the Author's personal website, university networks, or primary employer's 
institutional websites, and conference websites that feature presentations by the Author(s) provided the following conditions are met:

- Appropriate citation to the Published Work is given

- Modifications to the presentation of previously published data in figures and tables are noted and distinguished from any new data not contained in the Published Work, and

- Reuse is not to illustrate news stories unrelated to the Published Work

- Web posting by the Author(s) is for non-commercial purposes.

To reuse figures, tables, artwork, illustrations, and text from ACS Published Works in general, ACS requests that interested parties use the Copyright Clearance Center Rightslink service. For information see http://pubs.acs.org/page/copyright/rightslink.html

General ACS permission information can be found at http://pubs.acs.org/page/copyright/permissions.html.

3. Reuse in Teaching or In-House Training: In order to preserve the integrity of the scientific record, the Author(s) are encouraged to link to the Published Work using the ACS Articles on Request author-directed link as applicable for teaching and in-house training and this use is subject to the conditions identified below (see http://pubs.acs.org/page/policy/articlesonrequest/index.html). Regardless, the Author(s) may reproduce their Submitted, Accepted, or Published Work for instructional use in courses as a stand-alone handout, as part of a packet, or electronically for use by students enrolled in the course the Author is teaching as long as the following conditions are met: 
- Proper credit must be given to the Published Work and a link to the Published Work must be included using the ACS Articles on Request author-directed link (see http://pubs.acs.org/page/policy/articlesonrequest/index.html). The following notice should either be posted with or printed on all uses of the Accepted Work described in this clause: "This material is excerpted from a work that was [accepted for publication/published] in [Journal Title], copyright (C) American Chemical Society after peer review. To access the final edited and published work see [insert ACS Articles on Request author-directed link to Published Work, see http://pubs.acs.org/page/policy/articlesonrequest/index.html].”

- Electronic access must be provided via a password-protected website only to students enrolled in the course (i.e., not the general public). Availability to students should terminate when the course is completed.

- If a fee for distributed materials is charged for the use of Published Work in connection with the instructional use, prior written permission from the ACS must be obtained.

4. Presentation at Conferences: Subject to the ACS' "Ethical Guidelines to Publication of Chemical Research" (http://pubs.acs.org/ethics) and written confirmation (via letter or email) from the appropriate ACS journal editor to resolve potential conflicts with journal prior publication**/embargo policies, Authors may present orally or otherwise display all or part of the Submitted, Accepted, or Published Work in presentations at meetings or conferences. Authors may provide copies of the Submitted and Accepted Work either in print or electronic form to the audience. 
**Prior publication policies of ACS journals are posted on the ACS website at http://pubs.acs.org/page/policy/prior/index.html. Sharing of the Published Work with conference attendees is permitted if it is done either via the ACS Articles on Request author-directed link (see http://pubs.acs.org/page/policy/articlesonrequest/index.html) or in print. Audience recipients should be informed that further distribution or reproduction of any version of the Work is not allowed.

5. Share with Colleagues: Subject to the ACS' "Ethical Guidelines to Publication of Chemical Research" (http://pubs.acs.org/ethics), Authors may send or otherwise transmit electronic files of the Submitted or Accepted Work to interested colleagues prior to, or after, publication. Sharing of the Published Work with colleagues is permitted if it is done via the ACS Articles on Request author-directed link (see http://pubs.acs.org/page/policy/articlesonrequest/index.html). The sharing of any version of the Work with colleagues is only permitted if it is done for non-commercial purposes; that no fee is charged; and that it is not done on a systematic basis, e.g., mass emailings, posting on a listserv, etc. Recipients should be informed that further redistribution of any version of the Work is not allowed.

Authorized users of the ACS Publications website (http://pubs.acs.org/) may also email a link to the Author's article directly to colleagues as well as recommend and share a link to the Author's article with known colleagues through popular social networking services such as CiteULike, Digg, and Newsvine (see http://pubs.acs.org/sda/63224/index.html for more information). 
6. Posting Submitted Works on Websites and Repositories: A digital file of the Submitted Work may be made publicly available on websites or repositories (e.g., the Author's personal website, preprint servers, university networks or primary employer's institutional websites, third party institutional or subject-based repositories, and conference websites that feature presentations by the Author(s) based on the Submitted Work) under the following conditions:

- The Author(s) have received written confirmation (via letter or email) from the appropriate ACS journal editor that the posting does not conflict with journal prior publication/embargo policies (see http://pubs.acs.org/page/policy/prior/index.html).

- The posting must be for non-commercial purposes and not violate the ACS' "Ethical Guidelines to Publication of Chemical Research” (see http://pubs.acs.org/ethics).

- If the Submitted Work is accepted for publication in an ACS journal, then the following notice should be included at the time of posting, or the posting amended as appropriate: "This document is the unedited Author's version of a Submitted Work that was subsequently accepted for publication in [JournalTitle], copyright (C) American Chemical Society after peer review. To access the final edited and published work see [insert ACS Articles on Request author-directed link to Published Work, see http://pubs.acs.org/page/policy/articlesonrequest/index.html].”

If any prospective posting of the Submitted Work, whether voluntary or mandated by the Author(s)' funding agency, primary employer, or, in the case of Author(s) employed in academia, university administration, would violate any of the above conditions, the Submitted Work may not be posted. In these cases, Author(s) may either sponsor the 
immediate public availability of the final Published Work through participation in the fee-based ACS AuthorChoice program (for information about this program see http://pubs.acs.org/page/policy/authorchoice/index.html) or, if applicable, seek a waiver from the relevant institutional policy.

7. Posting Accepted and Published Works on Websites and Repositories: A digital file of the Accepted Work and/or the Published Work may be made publicly available on websites or repositories (e.g., the Author's personal website, preprint servers, university networks or primary employer's institutional websites, third party institutional or subject-based repositories, and conference websites that feature presentations by the Author(s) based on the Accepted and/or the Published Work) under the following conditions:

- It is mandated by the Author(s)' funding agency, primary employer, or, in the case of Author(s) employed in academia, university administration.

- For mandates from non-governmental institutions (e.g., universities, private sector corporations, non-governmental organizations, etc.), the Author(s) have received written confirmation (via letter or email) from the appropriate ACS journal editor that the posting does not conflict with journal prior publication policies (see http://pubs.acs.org/page/policy/prior/index.html).

- If the mandated public availability of the Accepted Manuscript is sooner than 12 months after online publication of the Published Work, a waiver from the relevant institutional policy should be sought. If a waiver cannot be obtained, the Author(s) may 
sponsor the immediate availability of the final Published Work through participation in the ACS AuthorChoice program - for information about this program see http://pubs.acs.org/page/policy/authorchoice/index.html.

- If the mandated public availability of the Accepted Manuscript is not sooner than 12 months after online publication of the Published Work, the Accepted Manuscript may be posted to the mandated website or repository. The following notice should be included at the time of posting, or the posting amended as appropriate: "This document is the Accepted Manuscript version of a Published Work that appeared in final form in [JournalTitle], copyright $\mathbb{C}$ American Chemical Society after peer review and technical editing by the publisher. To access the final edited and published work see [insert ACS Articles on Request author-directed link to Published Work, see http://pubs.acs.org/page/policy/articlesonrequest/index.html]."

- The posting must be for non-commercial purposes and not violate the ACS' "Ethical Guidelines to Publication of Chemical Research" (see http://pubs.acs.org/ethics).

- Regardless of any mandated public availability date of a digital file of the final Published Work, Author(s) may make this file available only via the ACS AuthorChoice Program. For more information, see http://pubs.acs.org/page/policy/authorchoice/index.html.

Author(s) may post links to the Accepted Work on the appropriate ACS journal website if the journal posts such works.

Author(s) may post links to the Published Work on the appropriate ACS journal website using the ACS Articles on Request author-directed link (see 
http://pubs.acs.org/page/policy/articlesonrequest/index.html).

Links to the Accepted or Published Work may be posted on the Author's personal website, university networks or primary employer's institutional websites, and conference websites that feature presentations by the Author(s). Such posting must be for non-commercial purposes.

SECTION III: Retained and Other Rights

1. Retained Rights: The Author(s) retain all proprietary rights, other than copyright, in the Submitted Work. Authors should seek expert legal advice in order to secure patent or other rights they or their employer may hold or wish to claim.

2. Moral Rights: The Author(s) right to attribution and the integrity of their work under the Berne Convention (article 6bis) is not compromised by this agreement.

3. Extension of Rights Granted to Prior Publications: The rights and obligations contained in Section II: Permitted Uses by Author(s), Section III: Retained and Other Rights, and Appendix A, Section I: Author Warranties and Obligations of this agreement are hereby extended to the Author(s)' prior published works in ACS journals. 


\section{Appendix D-1 Copyright Permission for Chapter 6}

The content of Chapter 6 was originally published in TMS Annual Meeting, Extraction and Processing Division - 2012 EPD Congress - Held During the TMS 2012 Annual Meeting and Exhibition (a Minerals, Metals and Materials Society conference proceedings). According to the regulations in the Copyright Form of the Minerals, Metals and Materials Society, the author retains the right to reproduce the published work in his Ph.D. dissertation. The Copyright Form can be accessed at the following website: http://www.tms.org/pubs/books/instructions/Copyright_Form.pdf. A copy of the Rights of Authors in the Copyright Form is attached below.

Title: $\quad$ Heat Transfer Characteristics of Magnetite under Microwave Irradiation

Authors: Zhiwei Peng, Jiann-Yang Hwang, Matthew Andriese, Zheng Zhang, Xiaodi Huang.

Publication: TMS Annual Meeting, Extraction and Processing Division - 2012 EPD Congress - Held During the TMS 2012 Annual Meeting and Exhibition

Year: $\quad 2012$

Pages: $\quad 121-128$

Copyright: (C) 2012 The Minerals, Metals and Materials Society 


\section{PART A. COPYRIGHT TRANSFER}

Copyright, title, interest, and all right in the manuscript named above is hereby transferred to TMS, effective when the manuscript is accepted for publication. This assignment and transfer applies to any other publication of the Society in addition to the publication designated.

THE AUTHOR(S), OR THE EMPLOYER(S) IN THE CASE OF WORKS MADE FOR HIRE, RETAIN THE FOLLOWING RIGHTS:

1.) All proprietary rights, other than copyright, such as patent rights.

2.) The right to use all or portions of the above paper in oral presentations or other works.

3.) The right to make limited distribution of the article or portions thereof prior to publication.

4.) Royalty-free permission to reproduce the above paper for personal use or, in the case of a work made for hire, the employer's use, provided that a.) the source and TMS copyright are indicated, b.) the copies are not used in a way that implies endorsement by TMS of a product or service, and c.) the copies are not offered for sale.

5.) In the case of work performed under U.S. government contract, TMS grants the U.S. government royalty-free permission to reproduce all or portions of the paper, and to authorize others to do so for U.S. government purposes. 


\section{Appendix E-1 Copyright Permission for Chapter 7}

A part of Chapter 7 was originally published in ISIJ International (an Iron and Steel Institute of Japan journal). Permission has been granted to the author who reused the published work in his Ph.D. dissertation. A copy of the permission is attached below.

Title: Numerical Simulation of Heat Transfer during Microwave Heating of Magnetite

Authors: Zhiwei Peng, Jiann-Yang Hwang, Matthew Andriese, Wayne Bell, Xiaodi Huang, Xinli Wang.

Publication: ISIJ International

Year: 2011

Volume: $\quad 51$

Issue: $\quad 6$

Pages: $\quad$ 884-888

Copyright: (C) 2011 The Iron and Steel Institute of Japan 
To: ISIJ International

\section{From Zhiwei Peng}

\section{Request for permission to reproduce copyrighted material}

Dear ISIJ International Editor,

I am preparing a review article entitled:

Heat Transfer in Microwave Heating (A Doctoral Dissertation)

For submission to Graduate School of Michigan Technological University published by Michigan Technological University

I wish to have your permission to include in my article the following material: Full text

From the article written by Zhiwei Peng, Jiann-Yang Hwang, Matthew Andriese, Wayne Bell, Xiaodi Huang and Xinli Wang

in the publication "Numerical Simulation of Heat Transfer during Microwave Heating of Magnetite" ISIJ Int., 51 (2011), 884.

If permission is granted for the use of this material, the author(s) and your publication will be credited as the source. If you would like the credit line to take any special form, please let me know what this should be.

I should be greatly appreciated if you would indicate your agreement by signing and returning one copy this form. Thank you for your cooperation.

Sincerely yours,
\begin{tabular}{ll} 
Name $\quad$ Zhiwei Peng \\
\hline Fax $\quad+1-9064872934$ \\
\hline E-mail zpeng@mtu.edu
\end{tabular}

Permission granted to reproduce the material specified above:

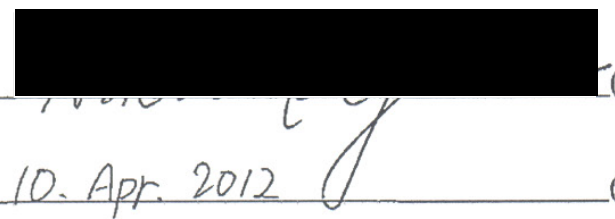

(Signature of copyright holder/author)

Credit line to be used: 


\section{Appendix E-2 Copyright Permission for Chapter 7}

A part of Chapter 7 was originally published in Metallurgical and Materials Transactions A (a Minerals, Metals and Materials Society journal). According to the regulations in the Copyright Form of the Minerals, Metals and Materials Society, the author retains the right to reproduce the published work in his Ph.D. dissertation. A copy of the Rights of Authors in the Copyright Form is attached below.

Title: Numerical Analysis of Heat Transfer Characteristics in Microwave Heating of Magnetic Dielectrics

Authors: Zhiwei Peng, Jiann-Yang Hwang, Chong-Lyuck Park, Byoung-Gon Kim, Gerald Onyedika.

Publication: Metallurgical and Materials Transactions A

Year: $\quad 2012$

Volume: $\quad 43 \mathrm{~A}$

Issue: $\quad 3$

Pages: $\quad$ 1070-1078

Copyright: $\quad$ (C) 2012 The Minerals, Metals and Materials Society 


\section{PART A. COPYRIGHT TRANSFER}

Copyright, title, interest, and all rights in the manuscript named above are hereby transferred to TMS and ASM International, effective when the manuscript is accepted for publication. This assignment and transfer applies to any other subsequent publication of either organization in addition to the publication designated, provided that proper acknowledgement is made.

THE AUTHOR(S), OR THE EMPLOYER(S) IN THE CASE OF WORKS MADE FOR HIRE, RETAIN THE FOLLOWING RIGHTS:

1.) All proprietary rights, other than copyright, such as patent rights.

2.) The right to use all or portions of the above paper in oral presentations or other works.

3.) The right to make limited distribution of the article or portions thereof prior to publication.

4.) Royalty-free permission to reproduce the above paper for personal use or, in the case of a work made for hire, the employer's use, provided that a.) the source and copyright are indicated, b.) the copies are not used in a way that implies endorsement by TMS and ASM International of a product or service, and c.) the copies are not offered for sale.

5.) In the case of work performed under U.S. government contract, TMS and ASM International grants the U.S. government royalty-free permission to reproduce all or portions of the paper, and to authorize others to do so for U.S. government purposes. 


\section{Appendix F-1 Copyright Permission for Chapter 8}

The content of Chapter 8 was accepted for publication in TMS Annual Meeting, 4th International Symposium on High-Temperature Metallurgical Processing - Held During the TMS 2013 Annual Meeting and Exhibition (a Minerals, Metals and Materials Society conference proceedings). According to the regulations in the Copyright Form of the Minerals, Metals and Materials Society, the author retains the right to reproduce the published work in his Ph.D. dissertation. The Copyright Form of the Minerals, Metals and Materials Society can be accessed at the following website: http://www.tms.org/pubs/books/instructions/Copyright_Form.pdf. A copy of the Rights of Authors in the Copyright Form is attached below.

Title: $\quad$ Microwave Reflection loss of Ferric Oxide

Authors: Zhiwei Peng, Jiann-Yang Hwang, Byoung-Gon Kim, Matthew Andriese, Xinli Wang.

Publication: TMS Annual Meeting, 4th International Symposium on HighTemperature Metallurgical Processing - Held During the TMS 2013 Annual Meeting and Exhibition

Year: $\quad 2013$

Copyright: $\quad$ (C) 2013 The Minerals, Metals and Materials Society 


\section{PART A. COPYRIGHT TRANSFER}

Copyright, title, interest, and all right in the manuscript named above is hereby transferred to TMS, effective when the manuscript is accepted for publication. This assignment and transfer applies to any other publication of the Society in addition to the publication designated.

THE AUTHOR(S), OR THE EMPLOYER(S) IN THE CASE OF WORKS MADE FOR HIRE, RETAIN THE FOLLOWING RIGHTS:

1.) All proprietary rights, other than copyright, such as patent rights.

2.) The right to use all or portions of the above paper in oral presentations or other works.

3.) The right to make limited distribution of the article or portions thereof prior to publication.

4.) Royalty-free permission to reproduce the above paper for personal use or, in the case of a work made for hire, the employer's use, provided that a.) the source and TMS copyright are indicated, b.) the copies are not used in a way that implies endorsement by TMS of a product or service, and c.) the copies are not offered for sale.

5.) In the case of work performed under U.S. government contract, TMS grants the U.S. government royalty-free permission to reproduce all or portions of the paper, and to authorize others to do so for U.S. government purposes. 


\section{Appendix G-1 Copyright Permission for Chapter 9}

The content of Chapter 9 was originally published in Applied Physics Express (a Japan Society of Applied Physics journal). According to the Copyright Transfer Agreement of the Japan Society of Applied Physics, the author retains the right to reproduce the published work in his Ph.D. dissertation. The Copyright Transfer Agreement of the Japan Society of Applied Physics can be accessed at the following website: http://apex.jsap.jp/pdf/copyrightform.pdf. A copy of the "Rights of Authors" in the Copyright Transfer Agreement is attached below.

Title: $\quad$ Magnetic Loss in Microwave Heating

Authors: Zhiwei Peng, Jiann-Yang Hwang, Matthew Andriese.

Publication: Applied Physics Express

Year: $\quad 2012$

Volume: $\quad 5$

Issue: $\quad 7$

Pages: $\quad$ 077301-077301-3

Copyright: C 2012 The Japan Society of Applied Physics 


\section{Copyright Transfer Agreement}

The undersigned hereby agree(s) to transfer the following Economic Rights to the Work to the Japan Society of Applied Physics (hereinafter referred to as "JSAP"), effective if and when the manuscript is accepted for publication by APEX Editorial Board.

Economic Rights: any and all rights including, but not limited to, right of reproduction (Article 21 of the Copyright Law of Japan (hereinafter referred to as the "Law")), right of performance (Article 22 of the Law), right of presentation (Article 22-2 of the Law), right of public transmission (Article 23 of the Law), right of recitation (Article 24 of the Law), right of exhibition (Article 25 of the Law), right of distribution (Article 26 of the Law), right of transfer of ownership (Article 26-2 of the Law), right of lending (Article 26-3 of the Law), right of translation and adaptation (Article 27 of the Law), and the right of the original Author regarding the exploitation of derivative works (Article 28 of the Law).

The Author(s) shall not exercise Moral Rights with respect to the Society. Moral Rights refer to the right to make the works public (Article 18 of the Law), right to determine the indication of the Author's name (Article 19 of the Law), right to preserve the integrity of the work (Article 20 of the Law).

The Author(s) retain(s) the following rights (the "Rights of Authors"'):

Rights of Author(s)

(1) The right to use a part of the Work in future works and derivatives prepared by or on behalf of the Author(s). 
(2) The right to make copies of all or part of the Work without further permission, for the purpose of his personal use but not for resale.

Author(s) shall not use any Economic Rights other than listed above without prior written consent of JSAP.

Notwithstanding the foregoing, the Author may use, translate, or adapt the manuscript without prior written consent of JSAP, to the extent that such use falls within the limitations (i.e., Articles 30 through 50 of the Law), except in the cases listed below:

\section{(A) Commercial purposes;}

(B) Duplicated or redundant contributions to other journals of an academic society or academic journals or communications, whatsoever, domestic or overseas; or

(C) Any other purpose deemed extravagant in light of fair academic practices.

(3) Exception. - The submitted manuscript has been authored by a contractor of the U.S. Government under Contract No.

Accordingly, the U.S. Government retains a nonexclusive royalty-free license to publish or reproduce the published form of this contribution, or allows others to do so, for U.S. Government purposes. Note that the rights the U.S. Government retains are not for commercial purposes.

For further information about The Copyright Policy of JSAP, see http://www.jsap.or.jp/english/link/copyright.html. 